\title{
Polybutadien und Butadien enthaltende Copolymere mit kontrollierter Kettenstruktur durch RAFT-Polymerisation
}

\author{
Dissertation \\ zur Erlangung des mathematisch-naturwissenschaftlichen Doktorgrades \\ „Doctor rerum naturalium“ \\ der Georg-August-Universität Göttingen
}

\author{
vorgelegt von \\ Björn Springer \\ aus \\ Berlin
}

Göttingen 2011 
Referent: Prof. Dr. Philipp Vana

Korreferent: Prof. Dr. Michael Buback

Tag der mündlichen Prüfung: 06.07.2011 


\section{Inhaltsverzeichnis}

1. Zusammenfassung .................................................................................................................... 1

2. Einleitung..........................................................................................................................................

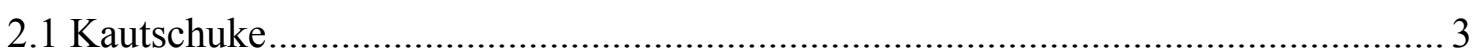

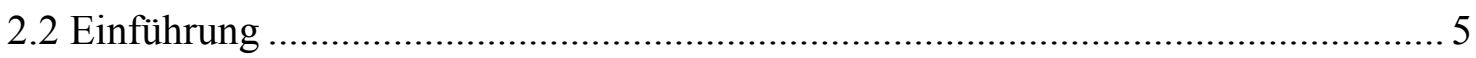

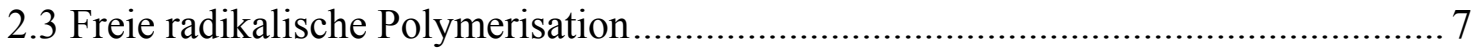

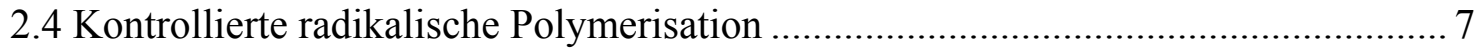

2.4.1 Nitroxide Mediated Polymerization (NMP) ................................................... 9

2.4.2 Übergangsmetall-katalysierter Atomtransfer (ATRP) .................................. 10

2.4.3 Degenerativer Kettentransfer (RAFT) ....................................................... 11

3. Theoretische Grundlagen ............................................................................... 13

3.1 Ideale Kinetik der konventionellen radikalischen Polymerisation ........................ 13

3.1.1 Kettenstart (Initiierung) ........................................................................ 14

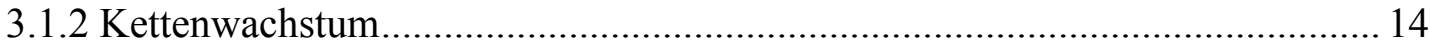

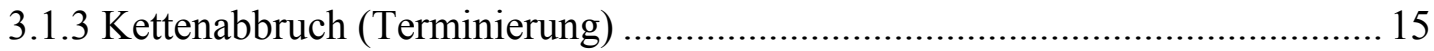

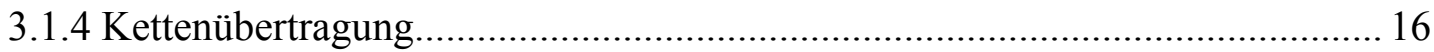

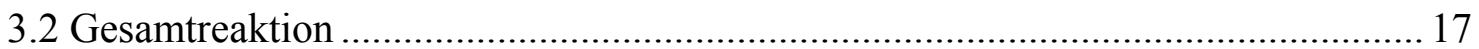

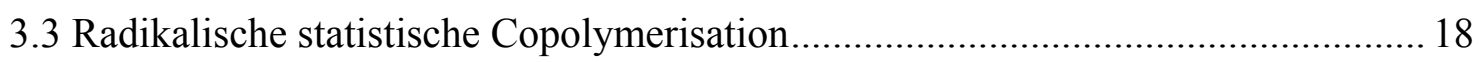

3.3.1 Kinetik der statistischen Copolymerisation ................................................. 18

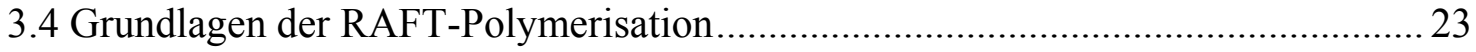

3.4.1 Mechanismus der RAFT-Polymerisation ..................................................... 23

3.4.2 Retardierung der Polymerisationsgeschwindigkeit..................................... 27

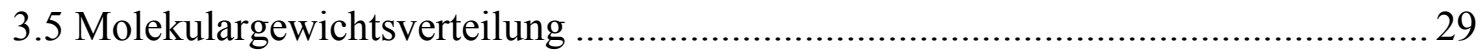

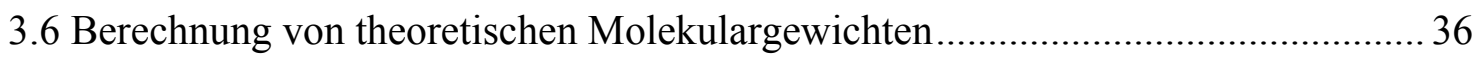

4. Experimenteller Aufbau I ................................................................................. 39

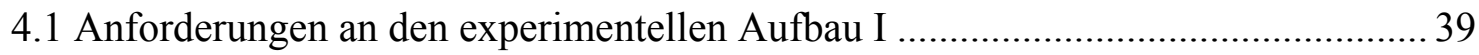

4.2. Hochdruck - Laborautoklav I „Modell II““............................................................ 40

4.2.1 Technische Daten und Beschreibung des Aufbaus .......................................... 40

4.2.2 Zubehör und Erweiterungen des experimentellen Aufbaus............................. 43

4.2.3 Homopolymerisationen von Butadien ........................................................... 50

4.2.4 Statistische Copolymerisation von Butadien(BD) mit Isopren (IP) und

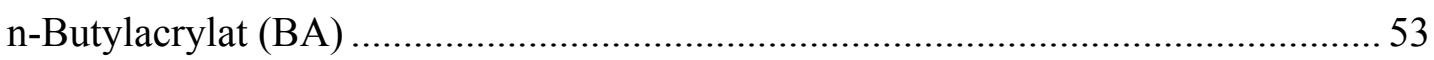

5. Experimenteller Aufbau Teil II ............................................................................. 55

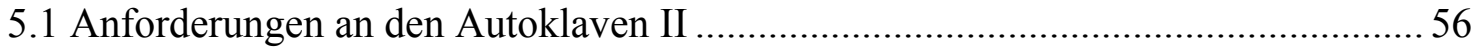




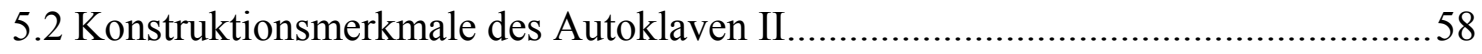

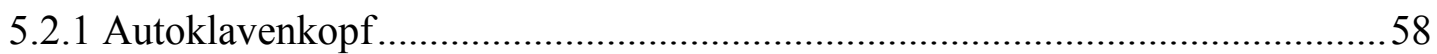

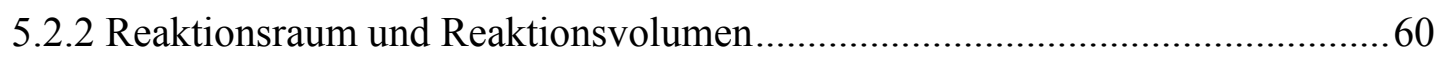

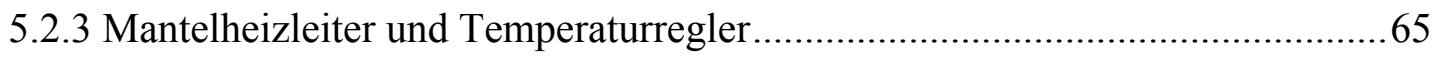

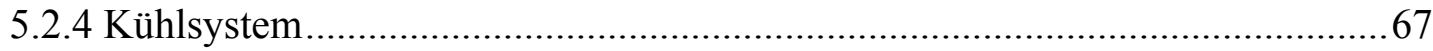

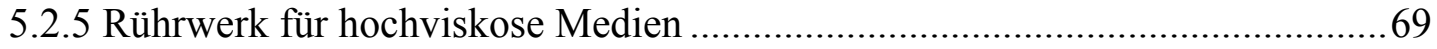

5.2.6 Probenentnahme während der Polymerisation ................................................ 71

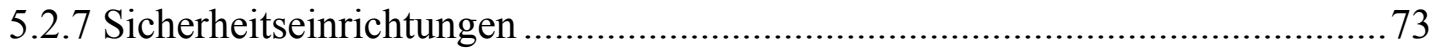

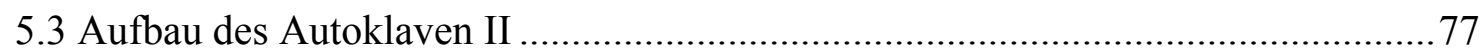

5.3.1 Montage des Rührwerks und der Magnetrührkupplung.................................78

5.3.2 Montage des Tauchrohrs, des Thermoelements und der Berstscheibe .............79

5.3.3 Montage des Manometers und des Ablassventils (K3)...................................79

5.3.4 Installation des Einschraubadapters (RZ3) und der Innenzelle.........................80

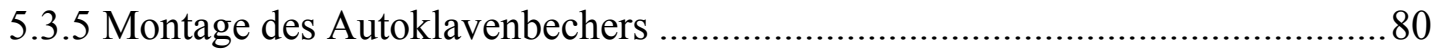

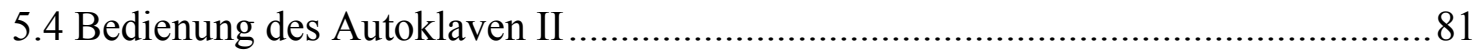

5.4.1 Abkühlen des Füllautoklaven und des Autoklavenbechers...............................8 82

5.4.2 Spülung des Füllautoklaven mit Stickstoff ..................................................... 82

5.4.3 Einkondensation von 1,3-Butadien (BD) in den Füllautoklaven ...................... 82

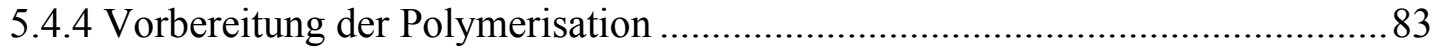

5.4.5 Spülung des Autolaven II mit Stickstoff ....................................................... 83

5.4.6 Einkondensation von 1,3-Butadien (BD) in den Autoklaven II.......................8 84

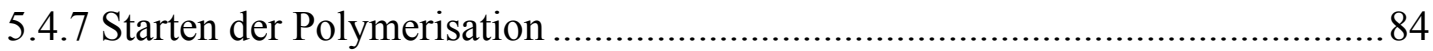

5.4.8 Probenentnahme während der Polymerisation ................................................. 84

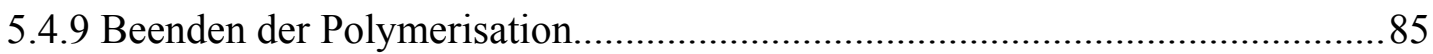

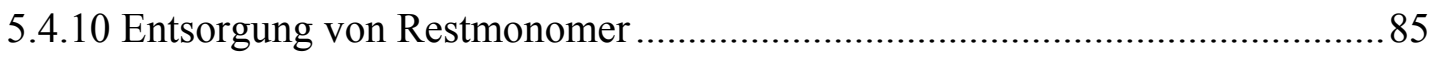

5.4.11 Entnahme der Polymerisationslösung …...................................................... 86

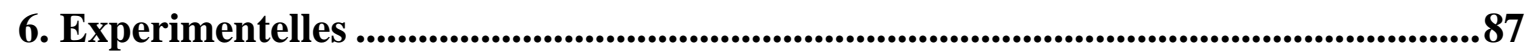

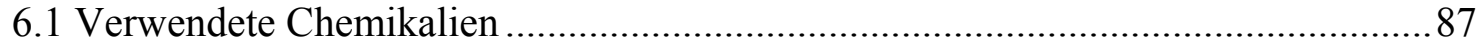

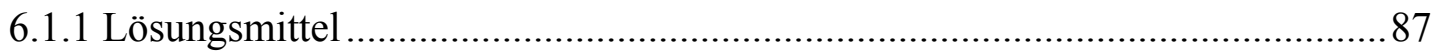

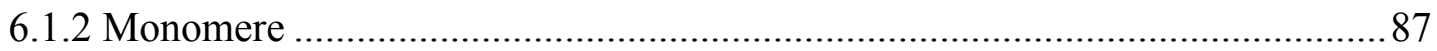

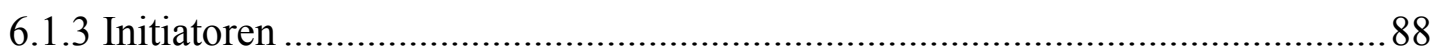

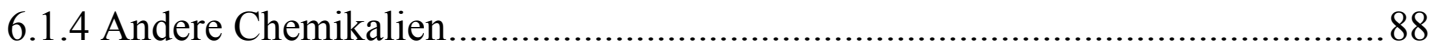

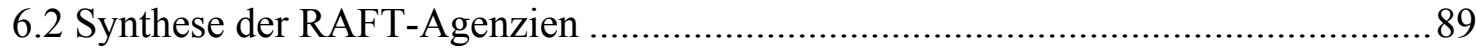




\section{Inhaltsverzeichnis}

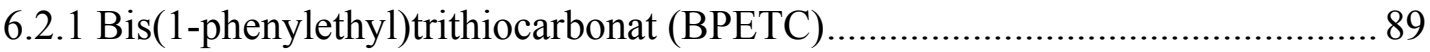

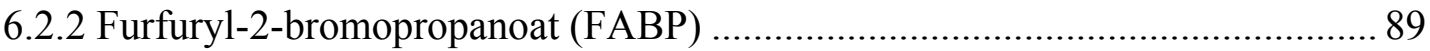

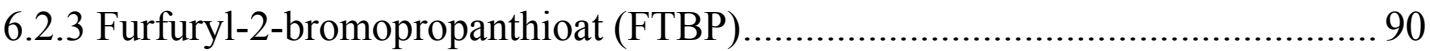

6.2.4 Natrium-(propyl)-trithiocarbonat (TTCP) .................................................. 91

6.2.5 Propyl-(furfurylpropanioat)-trithiocarbonat (FABPTTCP) …......................... 91

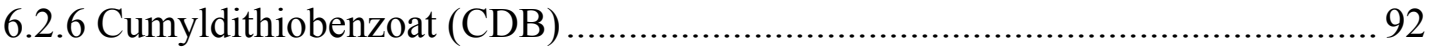

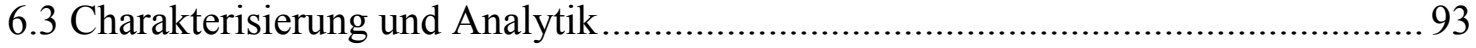

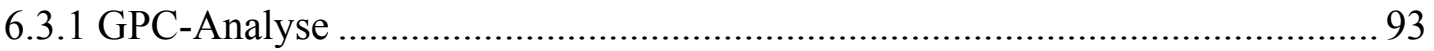

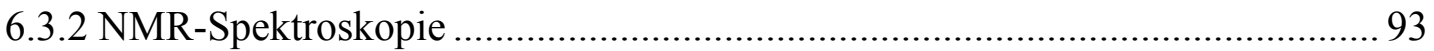

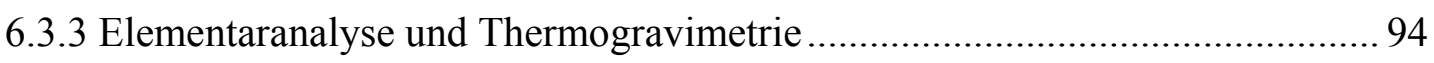

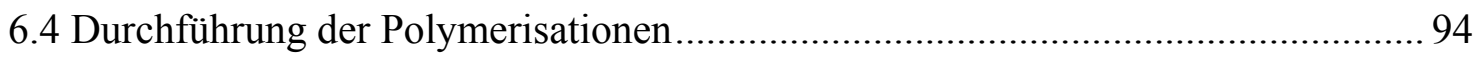

6.4.1 Polymerisationen im Thermoblock (Variante 1) …......................................... 94

6.4.2 Polymerisationen im Ölbad (Variante 2) ......................................................... 95

7. Auswertung der statistischen Copolymerisation.....................................................97

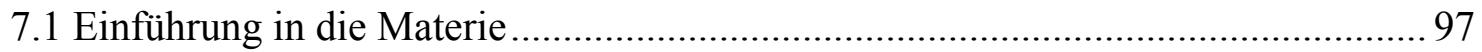

7.2 Statistische Copolymerisation von 1,3-Butadien (BD) und Butylacrylat (BA)...... 98

7.3 Statistische Copolymerisation von 1,3-Butadien (BD) und Isopren (IP) ............... 104

8. RAFT-Polymerisationen an Industrierußen ........................................................... 107

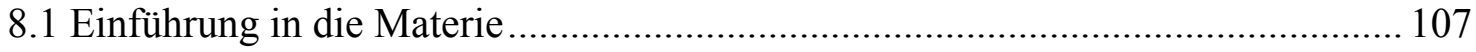

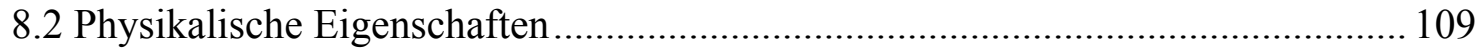

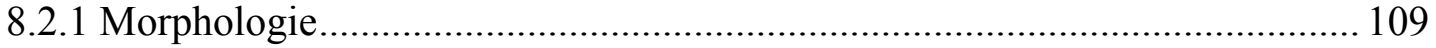

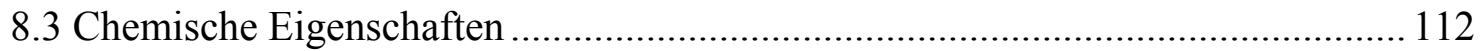

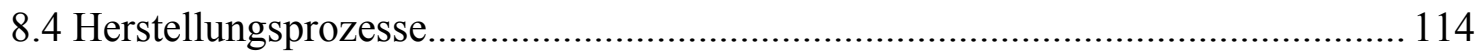

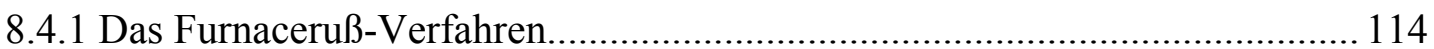

8.5 Verankerung von RAFT-Agenzien auf der Rußoberfläche ................................. 115

8.5.1 Abtrennung der Polymerketten von der Rußoberfläche ................................ 119

8.5.2 Abtrennung des Polymers in Lösung vom Polymer an der Rußoberfläche.... 121

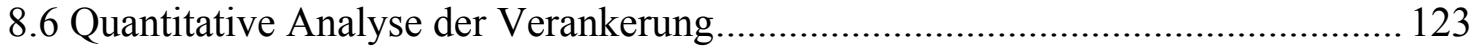

8.7 Konventionelle radikalische Polymerisationen in Gegenwart von Ruß ................ 129

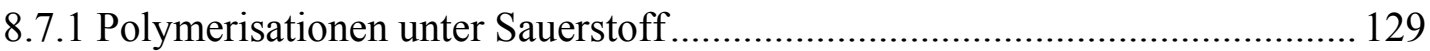

8.7.2 Polymerisationen unter Argon-Atmosphäre .................................................. 133

8.7.3 Polymerisationen unter Stickstoffatmosphäre ............................................. 135

8.8 Polymerisationen in Gegenwart der Ankergruppe „FTBP“‘................................ 137 
8.9 RAFT-Polymerisationen mit ungebundenem „FTBTTCP“ „................................. 142

8.10 RAFT-Polymerisationen in Gegenwart von Ruß ............................................... 146

8.10.1 RAFT-Polymerisationen mit „BPETC“...................................................... 146

8.10.2 RAFT-Polymerisationen mit „FABPTTCP“............................................. 149

8.11 RAFT-Polymerisation von der Rußoberfläche …..........................................152

8.11.1 RAFT-Polymerisation von der Oberfläche mit „FABPTTCP“.....................152

8.11.2 RAFT-Polymerisation von der Oberfläche mit „FTBPTTCP“ .....................156

8.12 Schlussfolgerung: Polymerisationen an Industrierußen ....................................162

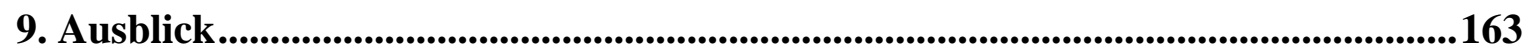

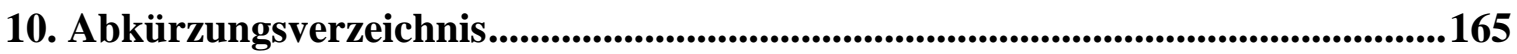

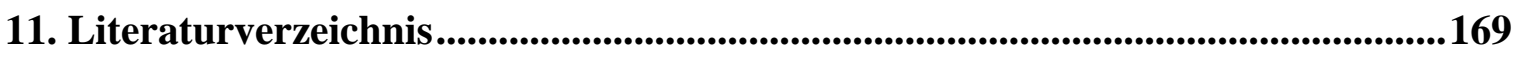




\section{Zusammenfassung}

Im Rahmen dieser Arbeit werden die Herstellung von Synthesekautschuken mit der Hilfe der RAFT-Polymerisation und die RAFT-Polymerisation von der Oberfläche von Industrierußen untersucht. Es werden neuartige Copolymerisationssysteme entwickelt und untersucht. Das Hauptaugenmerk liegt herbei auf einer erfolgreichen Polymerisationskontrolle der RAFT-Agenzien, um neuartige Copolymermaterialien herzustellen. Aus diesem Grund werden Polybutadien und 1,3-Butadien enthaltende Copolymere mit kontrollierter Kettenstruktur hergestellt. Exemplarisch werden Cumyldithiobenzoat (CDB) und Bis(1-phenylethyl)trithiocarbonat (BPETC) als RAFTAgenzien in Lösungsmittelpolymerisationen verwendet, um die Polymerisationskontrolle anhand der Charakteristika einer kontrollierten radikalischen Polymerisation zu verifizieren. Weiterhin wird die Mikrostruktur der Copolymere bestimmt. Die Polymerisationen werden im Satzbetrieb in einem Autoklaven durchgeführt, welcher sich bereits in vorherigen Untersuchungen bewährt hat. Um die rheologischen Eigenschaften dieser neuartigen Polymermaterialien untersuchen $\mathrm{zu}$ können, sind Probenmengen erforderlich, die den Labormaßstab übersteigen. Aufgrund der Erfahrungen im Umgang mit 1,3-Butadien wird im Rahmen dieser Arbeit ein erweiterter experimenteller Aufbau geplant und entwickelt. Die Anforderungen an die Apparatur und die technische Umsetzung des „Upscaling“ auf ein Reaktionsvolumen von $V=1900 \mathrm{~cm}^{3}$ werden detailliert betrachtet und beschrieben. Mit diesem neuentwickelten Autoklaven können Probenmengen hergestellt werden, welche eine Untersuchung der Polymereigenschaften auch im industriellen Maßstab ermöglichen.

Im weiteren Verlauf der Arbeit wird einen neue Möglichkeit der RAFT-Polymerisation von der Oberfläche von Industrierußen entwickelt und erstmalig untersucht. Hierzu wird die Diels-Alder-Reaktion als eine neue Verankerungstechnik getestet, um RAFT-Agenzien über die R-Gruppe an die Oberfläche zu binden. Neuartige RAFTAgenzien werden synthetisiert, um eine Verankerung über eine Diels-Alder-Reaktion zu ermöglichen. Hierbei wird auf einen einfachen und gut zu reproduzierenden Syntheseweg geachtet, da die RAFT-Agenzien für die Polymerisationen im großen Autoklaven in beachtlichen Mengen zur Verfügung stehen müssen. Ebenso wird in der Struktur der RAFT-Agenzien eine Ester- bzw. Thioesterfunktionalität eingebaut, um eine spätere 
Abspaltung des Polymers von der Oberfläche zu ermöglichen. Die Quantifizierung der Verankerungsreaktion erfolgt durch thermogravimetrische Messungen und Elementaranalyse. Weiterhin werden im Rahmen von Voruntersuchungen konventionelle radikalische Polymerisationen von Styrol in Gegenwart von Industrierußen durchgeführt, um den Einfluss auf den Umsatz-Zeit-Verlauf oder auf die Entwicklung der Molekulargewichte zu untersuchen. Neben der Betrachtung der konventionellen radikalischen Polymerisation werden auch Lösungsmittelpolymerisationen von Styrol in Toluol mit ungebundenem RAFT-Agenzien in Gegenwart von Ruß untersucht. Im Laufe dieser Experimente wird eine optimierte Aufarbeitung des heterogenen Polymerisationsansatzes entwickelt, um eine Analyse des Polymers mit Hilfe der Gel-Permations-Chromatographie (GPC) zu ermöglichen. Weiterhin wird die Polymerisationskontrolle der neuen RAFT-Agenzien betrachtet. Schließlich werden RAFT-Polymerisation von Styrol von der Rußoberfläche mit gebundenen RAFT-Agenzien durchgeführt. Die erfolgreiche Polymerisationskontrolle von der Rußoberfläche wird durch die Entwicklung der Molekulargewichte mit dem Umsatz bestätigt. In diesem Zusammenhang wird die Beladungsdichte der Rußpartikel mit Polymerketten an der Oberfläche durch thermogravimetrische Messungen quantifiziert. Die Analyse der Rußpartikel zeigt eine Zunahme der Beladungsdichte mit der Polymerisationszeit bzw. dem Umsatz. Im Rahmen dieser Arbeit werden grundlegende Untersuchungen der RAFT-Polymerisation an der Oberfläche von Industrierußen durchgeführt. Die neuartige Verankerungstechnik, die Möglichkeit der gezielten Abspaltung der Polymerketten und die einfache Synthese der RAFT-Agenzien sind wegweisend für die Herstellung und für die systematische Untersuchung von neuartigen Hybridmaterialien in der Kautschukindustrie. 


\section{Einleitung}

Polymere oder Makromoleküle finden in unserer Gesellschaft ein immer größeres und vielfältigeres Einsatzgebiet. Im allgemeinen Sprachgebrauch auch als „Kunststoffe“ oder „Plastik“ bezeichnet, stellen Produkte aus synthetischen Polymeren einen beachtlichen Anteil an unseren alltäglichen Gebrauchsgegenständen dar.

Allerdings ist die Weltproduktion an synthetischen Polymeren (2,3 $10^{8}$ Tonnen),

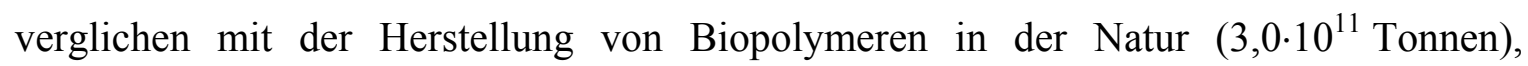
bescheidend gering. Allein der jährliche Verbrauch an Biomasse in Form von Nahrung und Holz beträgt ca. 7,1·10 Tonnen $^{[1]}$. Es scheint also erstrebenswert den Verbrauch und die Nutzung von immer mehr regenerativen Biopolymeren zu intensivieren und somit die Verschwendung von fossilen Rohstoffen $\mathrm{zu}$ verringern. Allerdings können die physikalischen Eigenschaften dieser Biopolymere nur in einem sehr kleinen Bereich variiert werden.

In der modernen Polymerchemie können die Eigenschaften der synthetischen Polymere durch verschiedene Herstellungsverfahren und Aufarbeitungsvarianten in einem erheblichen Maße gesteuert und für den späteren Verwendungszweck optimiert werden. So ist es nicht verwunderlich, dass es zur Verwendung von Naturkautschuk zahlreiche synthetische Alternativen gibt.

\subsection{Kautschuke}

Der deutsche Name „Kautschuk“ leitet sich vom indianischen Wort „Cahuthu“ (tränender Baum) ab. Kautschuk fand $\mathrm{zu}$ Anfang des 19. Jahrhunderts in Europa größere Verwendung, da die Verarbeitung des Kautschuks und dessen Produkte bis zu diesem Zeitpunkt noch nicht ausgereift waren.

Kautschuk ist ein vernetzbares Polymer mit gummielastischen Eigenschaften bei Raumtemperatur. Unter Einfluss von Deformationskräften oder höheren Temperaturen beginnt Kautschuk zu fließen und kann somit formgebend verarbeitet werden. Er dient zur Herstellung von Elastomeren, Reifen, Transportbändern, Schuhwerk und elastischen Materialien aller Art.

1820 entwickelte Thomas Hancock eine Apparatur, welche es ermöglichte Kautschuk durch Scherung zwischen zwei Rotoren zu erweichen und so die Weiterverarbeitung zu 
erleichtern. Diese Mastikation war der erste lösungsmittelfreie Weg der Kautschukaufarbeitung, wobei das mittlere Molekulargewicht des Polymers durch oxidative Spaltung verkleinert wurde. Dieser Arbeitsschritt wird auch heute noch in der Kautschukindustrie durchgeführt. Natürlich vorkommender Kautschuk ist bei tieferen Temperaturen steif und unflexibel. Erst bei höheren Temperaturen erweicht er und wird somit klebrig. 1839 entwickelte Charles Goodyear das Verfahren der Vulkanisation. Er zeigte, dass Erhitzen mit Schwefel in Anwesenheit von Bleiweiß zu einer Vernetzung des Kautschuks führt. Der Kautschuk besitzt nach der Vulkanisation eine verbesserte Elastizität und eine höhere Lebensdauer als der Rohkautschuk. Somit konnten völlig neue Anwendungsbereiche erschlossen werden, welche die Nachfrage an Naturkautschuk drastisch erhöhten und die gezielte Optimierung von Kautschukprodukten vorantrieben. Im Jahre 1904 wurde erstmals Ruß als Füllstoff in den Naturkautschuk eingebracht, wodurch sich einige mechanische Eigenschaften weiter verbesserten. Somit konnte in der Produktion von Luftreifen den immer leistungsstärkeren Motorfahrzeugen Rechnung getragen werden. Die steigende Nachfrage nach Naturkautschuk, die aufwändige Gewinnung und die weiten Transportwege führten $\mathrm{zu}$ immer drastischeren Preiserhöhungen. Auch die dezentrale Lage der Hauptproduktionsstätten von Naturkautschuk zum europäischen Kontinent steigerten die Bemühungen und das Interesse nach synthetischen Alternativen zum teilweise kriegswichtigen Naturprodukt.

1906 begannen die Farbenfabriken Bayer auf Vorschlag von F. Hofmann Isopren zu polymerisieren und stellten somit den ersten Synthesekautschuk in einem Warmpolymerisationsverfahren her. Dieses wurde im Jahre 1909 zum Patent angemeldet. Im Jahre 1910 wurde die alkalimetallkatalysierte Dienpolymerisation entwickelt und somit das erste großtechnisch interessante Verfahren vorgestellt. Da die Synthesen von Butadien und Isopren aufwändig waren, wurde als Ausgangsprodukt das gut zugängliche 2,3-Dimethyl-Butadien verwendet. In den Kriegsjahren von 1914 bis 1918 produzierte die IG Farbenindustrie 150 Tonnen des Methylkautschuks.

In den 20er Jahren des letzten Jahrhunderts konzentrierten sich die Forschungen auf die Polymerisation von Butadien, da das dabei entstehende Polymer bessere physikalische Eigenschaften besaß. Der Synthesekautschuk „BUNA“ wurde durch die Natrium-initiierte Massepolymerisation von Butadien großtechnisch hergestellt. Ende der 20er Jahre wurde ein Emulsionsprozess zur Copolymerisation von Butadien mit Styrol (BUNA S) oder Acrylnitril (BUNA N) entwickelt. 


\section{Einleitung}

Die Entdeckung der metallorganisch katalysierten Niederdruckpolymerisation von Ethylen durch Ziegler 1953 eröffnete auch der Synthesekautschukforschung neue Möglichkeiten und Anwendungsgebiete. Kurz danach konnten Isopren und Butadien erstmals unter Kontrolle der Konfiguration zu hoch-cis-1,4-Produkten polymerisiert werden. Die Weiterentwicklung der katalytischen Lösungsmittelpolymerisation ermöglichte in den Folgejahren die Herstellung von optimierten Synthesekautschuken, welche in zahlreichen Anwendungsgebieten den Naturkautschuk ergänzen oder gar ersetzen konnten.

Ungefähr die Hälfte der gesamten Kautschukproduktion wird für Fahrzeugreifen verwendet und kann der Klasse der Allzweckkautschuke zugeordnet werden. Die synthetischen Kautschuke Polybutadien (BR), Polyisopren (IR) und das statistische Styrol-Butadien-Copolymer (SBR), sowie Naturkautschuk (NR) bilden den Hauptbestandteil der Gummimischungen in der Reifenproduktion. An den modernen Fahrzeugreifen werden, bedingt durch die großen Krafteinwirkungen, hohe Ansprüche gestellt. Wesentlich für die Anwendungen sind gute mechanische Eigenschaften über einen weiten Temperaturbereich bei gleichzeitig geringer Erwärmung durch dynamische Belastungen. Je nach Reifentyp oder Verwendungszweck rücken zusätzliche Anforderungen, wie hoher Abriebwiderstand, gutes Traktionsverhalten und Gasdichtigkeit in den Vordergrund. Der Reifen muss eine hohe Zugfestigkeit aufweisen und gleichzeitig auf Scherung und Biegung flexibel reagieren. All diesen besonderen Beanspruchungen wird der Reifen einerseits durch den Aufbau, andererseits durch die Verwendung von bis zu 16 verschiedenen Kautschukmischungen gerecht.

Die Verringerung des Rollwiderstandes und somit des Benzinverbrauchs sind heute ein vorrangiges Entwicklungsziel, um den steigenden ökonomischen und ökologischen Ansprüchen gerecht $\mathrm{zu}$ werden. Der Rollwiderstand wird in erster Linie durch die Wechselwirkungen zwischen Kautschuk und Füllstoffen (z.B. Ruß oder Silica) beeinflusst. Diese Wechselwirkungen hängen, neben der Oberflächenaktivität des Füllstoffes auch vom chemischen Aufbau der Kautschuke und deren Mikrostruktur ab.

\subsection{Einführung}

Die kontrollierten Polymerisationen ermöglichen einen gezielten Aufbau von polymeren Architekturen und eine Kontrolle der Molekulargewichtsverteilung (Abbildung 2.1). Unter Annahme einer gleichzeitigen Aktivierung der Kontrollagenzien wächst die 
Molmasse kontinuierlich mit dem Anstieg des Umsatzes der Polymerisation. Auch lassen sich im Gegensatz zu konventionellen Polymerisationsformen schmalere Molekulargewichtsverteilungen realisieren. Synthetische Polymere haben eine gewisse Verteilung in den Molekulargewichten, d.h. die Kettenlängen der Polymere variieren zu einem gewissen Grad. Diese Verbreiterung in der Verteilung hat einen entscheidenden Einfluss auf die rheologischen Eigenschaften des Polymers. Weiterhin bieten lebende Polymerisationen einen leichteren Zugang zu hochverzweigten Polymeren, Stern- und Kammpolymeren.

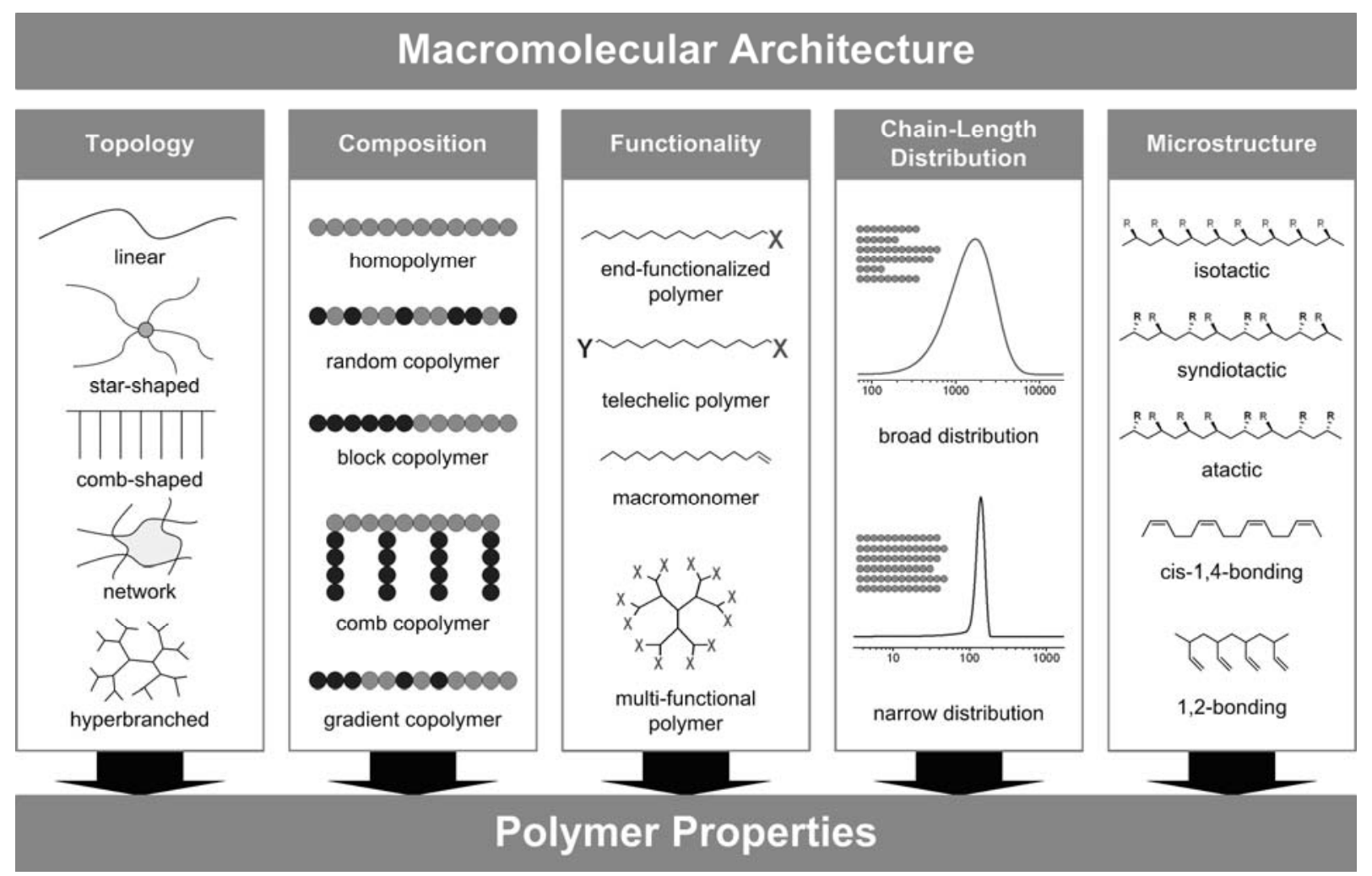

\begin{abstract}
Abbildung 2.2.1
Übersicht über die Polymerarchitekturen, welche mittels kontrollierter Polymerisationsformen umgesetzt werden können.
\end{abstract}

Auch lässt sich die Copolymerzusammensetzung leichter variieren, um zum Beispiel Blockstrukturen entlang der Hauptkette zu ermöglichen. Bei lebenden Polymerisationen befinden sich am Ende der Polymerkette unterschiedliche funktionelle Gruppen, welche bei erneuter Aktivierung weitere Monomereinheiten anlagern können oder eine chemische Endgruppenmodifikation ermöglichen. Darüber hinaus kann auch auf die Taktizität 


\section{Einleitung}

Einfluss genommen und somit die Mikrostruktur des Polymers direkt gesteuert werden. In der Gesamtheit betrachtet, stellen lebenden Polymerisationsformen ein universelles und zweckmäßiges Mittel dar, um maßgeschneiderte Polymere mit überdurchschnittlichen Eigenschaften zu produzieren.

\subsection{Freie radikalische Polymerisation}

Im Jahre 1838 gelang es dem Franzose Henri Victor Regnault erstmals Polyvinylchlorid (PVC) herzustellen, indem er Vinylchlorid dem Sonnenlicht aussetzte. Auch in unserer heutigen Zeit wird zur Herstellung von Kunststoffen im industriellen Maßstab eine Polyreaktion bevorzugt, welche eine Vielzahl von unterschiedlichen Monomeren in einem weiten Zustandsbereich zu polymerisieren vermag und eine gewisse Unempfindlichkeit gegenüber Verunreinigungen besitzt. Diese evidenten Vorteile kann die konventionelle radikalische Polymerisation in sich vereinigen. Mehr als die Hälfte aller kommerziell hergestellten Polymere entstammen einer freien radikalischen Polymerisation. Besonders zu erwähnen sind in diesem Fall Massenkunststoffe wie Polyethylen mit niedriger Dichte (LDPE), Polystyrol (PS), Polyvinylchlorid (PVC) und Polymethylacrylat (Plexiglas).

Bei der freien radikalischen Polymerisation handelt es sich um eine Kettenreaktion. Der Kettenstart (Initiierung), das Kettenwachstum (Propagation) und der Kettenabbruch (Terminierung) erfolgen während der Polymerisation parallel. Die gewünschte Kettenlänge kann durch die Konzentration der initiierenden Spezies eingestellt werden und wächst nicht mit dem Umsatz.

\subsection{Kontrollierte radikalische Polymerisation}

Eine kontrollierte Herstellung von Polymermaterialien mit einer definierten Architektur und Funktionalität war immer ein Ziel der modernen Polymerchemie. Ziegler zeigte bereits im Jahre 1936, dass unter Verwendung von Alkyllithium als Initiator Styrol und Butadien kontrolliert anionisch polymerisiert werden können. Diese Polymerisation verläuft ohne Kettentransfer und Terminierung. In den 50er Jahren definierte Szwarc ${ }^{[2,3]}$ jede Polymerisation als lebend, bei der das mittlere Molekulargewicht $M_{\mathrm{n}}$ linear mit dem Umsatz steigt und Kettenübertragung und Kettenterminierung vernachlässigbar klein sind. Diese Definition greift allerdings nicht alle Charakteristika der lebenden Polymerisation auf. Nach IUPAC gilt, dass lebende Polymerisationen auch die Teilschritte der langsamen Initiierung, der reversiblen Bildung von aktiven Spezies mit verschiedenen Lebensdauern, 
der reversiblen Bildung von inaktiven Spezies und/oder der reversiblen Übertragung enthalten können.

Im Gegensatz zu anionischen Polymerisationen kommt es bei den radikalischen Polymerisationen auch zu Terminierungsreaktionen. Während sich zwei Anionen abstoßen, terminieren zwei Radikale immer diffusionskontrolliert. Aus diesem Grund kommt es zu sehr kurzen Radikallebensdauern. Ein Ansatz zur Erzeugung eines lebenden Charakters liegt in der Vermeidung der Terminierung. Es müsste in diesem Fall die effektive Radikalkonzentration heruntergesetzt oder die Radikalfunktionalität geschützt werden $^{[4-7]}$. Das Schützen der Radikalfunktionalität erfolgt durch ein Kontrollagens. Es liegen somit „haltbare“ Radikale vor.

Die Grundlage der kontrollierten radikalischen Polymerisation bildet das Gleichgewicht zwischen einer aktiven und inaktiven Spezies (Abbildung 2.4.1).

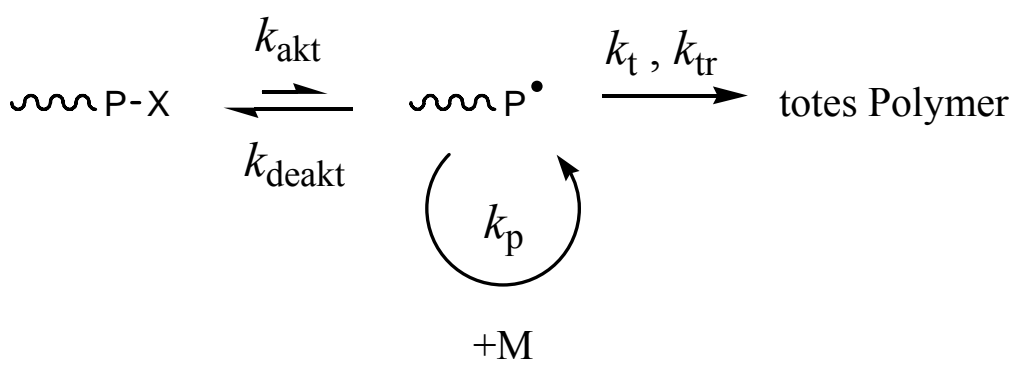

\section{Abbildung 2.4.1}

Schema des zentralen Gleichgewichts von aktiven und inaktiven Spezies bei kontrollierten radikalischen Polymerisationen.

Dieses dynamische Gleichgewicht verringert den Anteil der freien Radikale unter den reaktiven Spezies. Dadurch werden Kettenabbruchsreaktionen, welche das Kettenwachstum irreversibel beenden, unterbunden. Allerdings führt dieses Gleichgewicht auch zu einer Verlangsamung des Wachstums der individuellen Polymerketten. Im Gegenzug allerdings wird die Lebensdauer der reaktiven Spezies deutlich verlängert. 


\section{Einleitung}

Im Allgemeinen stehen folgende Charakteristika für eine „lebende“ oder kontrollierte Polymerisation:

- Die Anzahl der aktiven Zentren ist identisch mit der Anzahl der Polymerketten.

- Alle aktiven Zentren wachsen mit der gleichen Geschwindigkeit.

- Alle aktiven Zentren werden im Idealfall gleichzeitig aktiviert.

- Die Molekulargewichtsverteilung ist schmaler als im konventionellen Fall und entspricht im Idealfall einer Poisson-Verteilung.

- Die Molmasse wächst mit dem Umsatz.

Auch wenn die meisten Kriterien erfüllt werden können, lässt sich die Terminierung bei kontrollierten radikalischen Polymerisationen nie vollständig unterdrücken. Während der Anlagerung von Monomereinheiten an die reaktive Spezies, kann es zu irreversiblen Abbruchreaktionen kommen.

\subsubsection{Nitroxide Mediated Polymerization (NMP)}

Die Grundlage der kontrollierten radikalischen Polymerisation, das dynamische Gleichgewicht von aktiven und inaktiven Spezies, wird hier durch einen DissoziationsKombinations-Mechanismus realisiert (Abbildung 2.4.2). Der reversible Kettenabbruch erfolgt mit stabilen Radikalen, z.B. 2,2,6,6-Tetramethylpiperidinoxyl (TEMPO). Das Gleichgewicht liegt weit auf der Seite der schlafenden Spezies, wodurch die Konzentration an freien Radikalen sehr gering ist. Bevor die freie Radikalfunktionalität einer Polymerkette wieder reversibel terminiert wird, können einige Wachstumsschritte durchlaufen werden. Im Mittel ist die Anzahl der angelagerten Monomereinheiten für alle Polymerketten gleich. 


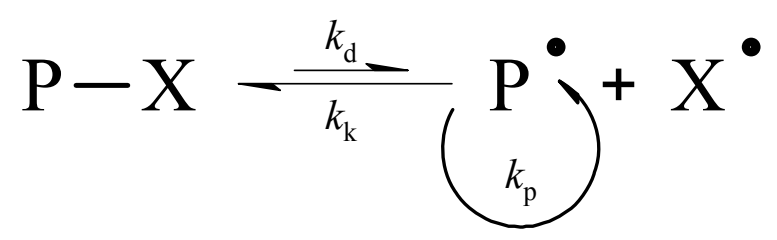

\begin{abstract}
Abbildung 2.4.2
Reversible Aktivierung durch Homolyse und Kombination. Aus der inaktiven Spezies wird durch homolytischen Bindungsbruch ein kohlenstoffzentriertes Radikal R und ein stabiles Radikal X (pesistent radical) gebildet.
\end{abstract}

Besondere Anforderungen werden an das stabile Radikal X gestellt, um das dynamische Gleichgewicht aufrecht zu erhalten. Es muss so stabil sein, dass es nicht zur Anlagerung von Monomereinheiten kommt oder ein Makromolekül durch Disproportionierung terminiert. Weiterhin darf es nicht mit sich selbst reagieren (persistent radicals) ${ }^{[8]}$. Da es allerdings auch zu irreversiblen Terminierungsreaktionen zwischen z.B. den wachsenden Polymerketten kommen kann, nimmt die Konzentration der stabilen Radikale X' während der Polymerisation kontinuierlich zu. Das dynamische Gleichgewicht verschiebt sich weiter auf die Seite der schlafenden Spezies, wodurch die Polymerisationsgeschwindigkeit weiter verlangsamt wird. Unter kinetischen Betrachtungen ist die Radikalkonzentration nicht mehr quasistationär und wird somit zeitabhängig.

\title{
2.4.2 Übergangsmetall-katalysierter Atomtransfer (ATRP)
}

Die ATRP ist eine weitere Möglichkeit, um Radikale vor der Terminierung zu schützen. In einem dynamischen Gleichgewicht kommt es durch eine Redoxreaktion zur Übertragung einer Spezies $\mathrm{X}$ auf einen Übergangsmetallkomplex in niedriger Oxidationsstufe, wobei eine radikale Spezies entsteht, welche Monomer im Wachstumsschritt anlagert. Der Übergangsmetallkomplex wird meistens durch mehrzähnige Liganden stabilisiert, um die Umsetzung von verschiedenen Oxidationsstufen zu ermöglichen. Die übertragende Spezies X entstammt in der Regel einem Alkylhalogenid. Bei der Rückreaktion des Gleichgewichts wird die radikale Spezies durch erneute Atomübertragung deaktiviert und ein polymeres Alkylhalogenid entsteht. Ein schneller Durchlauf dieses dynamischen Gleichgewichts erlaubt die Darstellung von Polymer mit einer sehr schmalen Molekulargewichtsverteilung. 


$$
\mathrm{P}_{\mathrm{i}}-\mathrm{X}+\mathrm{M}^{\mathrm{n} / \mathrm{L}_{\mathrm{x}}}-\frac{k_{\mathrm{d}}-}{k_{\mathrm{k}}}\left(\begin{array}{c}
\mathrm{P}^{\bullet} \\
k_{\mathrm{p}}
\end{array}\right)+\mathrm{X}-\mathrm{M}^{\mathrm{n}+1} / \mathrm{L}_{\mathrm{x}}
$$

\section{Abbildung: 2.4.3}

Schematische Darstellung der reversiblen Aktivierung bei der ATRP. Eine inaktive Spezies wird durch Atomübertragung auf einen Übergangsmetallkomplex in einer Redoxreaktion aktiviert.

Die ATRP unterliegt ebenso wie die NMP dem ,persistent radical effect“. Am Anfang liegt der Übergangsmetallkomplex nur in der kleineren Oxidationsstufe vor und wird erst im Laufe der Polymerisation gebildet. Während dieser ersten Reaktionsschritte ist die Konzentration an Radikalen sehr hoch, wodurch die Terminierung zweier Makroradikale vor der Deaktivierung durch Atomübertragung bevorzugt wird. Dadurch nimmt die Konzentration des Übergangmetalls $\mathrm{M}^{\mathrm{n}+1}$ kontinuierlich $\mathrm{zu}$, bis die Deaktivierung der radikalen Spezies schneller verläuft als die irreversible Terminierung zweier Makroradikale. $\mathrm{Zu}$ diesem Zeitpunkt verlangsamt sich die Geschwindigkeit der Polymerisation und die Kinetik erster Ordnung verläuft nichtlinear in Bezug auf den Monomerumsatz $^{[9]}$. Aufgrund der toxischen Wirkung ist die Verwendung von Übergangsmetallkomplexen bei der ATRP als nachteilig anzusehen. Eine vollständige Abtrennung der Übergangsmetallverbindungen ist in den meisten Fällen nicht gewährleistet.

\subsubsection{Degenerativer Kettentransfer (RAFT)}

Die RAFT-Polymerisation gehört auf dem Feld der kontrollierten radikalischen Polymerisationen $\mathrm{zu}$ den jüngeren Techniken und bietet eine große Vielfalt bei der Umsetzung von verschiedensten Monomeren und Reaktionsbedingungen ${ }^{[10,11]}$. Wie bei der NMP verzichtet die RAFT-Polymerisation auf die Verwendung von Übergangsmetallkomplexen. Allerdings ist die Grundlage des dynamischen Gleichgewichts einer kontrollierten radikalischen Polymerisation von beiden zuvor genannten Techniken verschieden. Abbildung 2.4.4 zeigt ein vereinfachtes Schema des Hauptgleichgewichts einer RAFT-Polymerisation. Der generative Kettentransfer der RAFT-Gruppe X erfolgt hierbei auf verschiedene aktive Spezies. Es erfolgt eine gleichzeitige Aktivierung und Deaktivierung, wodurch die effektive Radikalkonzentration 
nicht heruntergesetzt wird und sich somit die Polymerisationsgeschwindigkeit im Vergleich zur konventionellen radikalischen Polymerisation nicht vermindert.

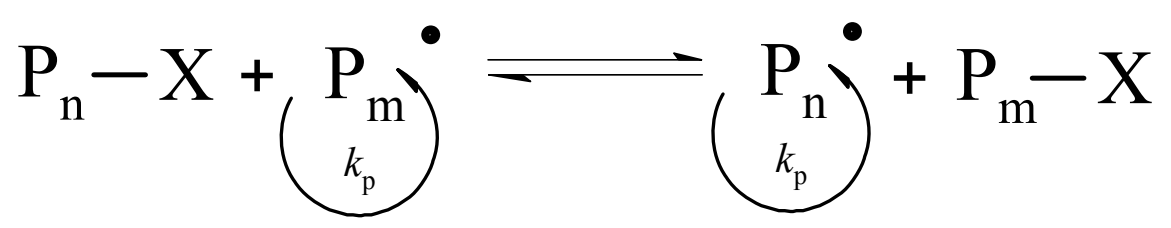

\section{Abbildung 2.4.4}

Schematische Darstellung eines vereinfachten Hauptgleichgewichts einer RAFT-Polymerisation. Der degenerative Kettentransfer überträgt die RAFT-Gruppe abwechselnd auf verschiedene aktive Spezies.

Weiterhin wird durch die dauerhafte reversible Kettenübertragung die Erscheinung des „persistent radical effects“ unterbunden. Unter der Annahme der kontinuierlichen Hintergrundinitiierung durch den Zerfall des Initiators, wird sogar nach der Terminierung zweier radikaler Zentren das dynamische Gleichgewicht erneuert. Kontrollierte radikalische Polymerisationsprozesse mit z.B. Dithioestern, Alkyliodiden oder Alkyltelluriden basieren auf dem Mechanismus des degenerativen Kettentransfers. Die Kinetik der RAFT-Polymerisation im Detail soll im Kapitel 3.3 näher betrachtet werden. 


\section{Theoretische Grundlagen}

In diesem Kapitel werden die theoretischen Grundlagen der idealen Polymerisationskinetik erläutert. Bevor die Gesamtpolymerisationsgeschwindigkeit betrachtet wird (Unterkapitel 3.2), erfolgt im Kapitel 3.1 die Beschreibung der einzelnen Elementarschritte der idealen Polymerisationskinetik einer konventionellen radikalischen Polymerisation. Im Anschluss wird im Abschnitt 3.3 die theoretische Betrachtung der statistischen radikalischen Copolymerisation behandelt. Als besonderer Vertreter der kontrollierten radikalischen Polymerisationstechniken wird im Abschnitt 3.4 die RAFT-Polymerisation näher erläutert. Im letzten Teil dieses Hauptkapitels werden die charakteristischen Größen und die Merkmale von Molekulargewichtsverteilungen beschrieben.

\subsection{Ideale Kinetik der konventionellen radikalischen Polymerisation}

Bei der Betrachtung der idealen Polymerisationskinetik werden folgende Annahmen getroffen $^{[12]}$ :

- Alle Reaktionen verlaufen irreversibel.

- Alle Polymerradikale besitzen die gleiche chemische Reaktivität, welche unabhängig von der Länge der Polymerketten ist.

- Das Monomer wird nur durch Wachstumsschritte verbraucht.

- Die Radikalkonzentration ist während der Polymerisation konstant (Bodenstein'sches Quasistationaritätsprinzip).

- Der Kettenabbruch kann nur durch Kombination oder Disproportionierung erfolgen.

Die kinetische Betrachtung der konventionellen radikalischen Homopolymerisation wird durch die obigen Annahmen vereinfacht und kann nun durch den Kettenstart (Initiierung), das Kettenwachstum (Propagation) und den Kettenabbruch (Terminierung) vollständig beschrieben werden. Allerdings müssen auch Kettenübertragungsreaktionen in Betracht gezogen werden, da diese Besonderheiten in der Molekulargewichtsverteilung hervorrufen und sich ebenfalls in den Produkteigenschaften widerspiegeln. 


\subsubsection{Kettenstart (Initiierung)}

Die Bildung von Radikalen erfolgt z.B. durch thermisch, chemisch oder photochemisch initiierte homolytische Bindungsspaltung eines Initiators I in zwei Initiatorradikale RI·

$$
\mathrm{I} \stackrel{k_{\mathrm{d}}, f}{\longrightarrow} 2 \mathrm{RI} \cdot
$$

\section{Gleichung 3.1.1}

In der Startreaktion erfolgt die erste Anbindung einer Monomereinheit an die Initiatorradikale, wodurch ein Monomerradikal (Primärradikal) entsteht. Bei thermischer Initiierung wird die Radikalbildungsgeschwindigkeit $R_{\mathrm{d}}$ mit dem Geschwindigkeitsgesetz einer Kinetik erster Ordnung beschrieben, wobei $c_{\mathrm{RI}}$ die Initiatorradikalkonzentration, $k_{\mathrm{d}}$ der Geschwindigkeitskoeffizient des Initiatorzerfalls, $f$ die Initiatoreffektivität und $c_{\mathrm{I}}$ die Initiatorkonzentration beschreibt.

$$
R_{\mathrm{d}}=\frac{\mathrm{d} c_{\mathrm{RI}}}{\mathrm{d} t}=2 \cdot k_{\mathrm{d}} \cdot f \cdot c_{\mathrm{I}}
$$

\section{Gleichung 3.1.2}

Die Initiatoreffektivität $f$ (Initiierungseffektivität) ist ein Korrekturfaktor. Er beschreibt den Anteil der gebildeten Initiatorradikale RI, welche am Start einer Polymerisation effektiv beteiligt sind. Die Radikalbildungsreaktion verläuft in den meisten Polymerisationen langsamer als die Anlagerung des ersten Monomermoleküls an das Initiatorradikal RI· Der Initiatorzerfall kann daher als geschwindigkeitsbestimmender Schritt in der Polymerisationskinetik angesehen werden.

\subsubsection{Kettenwachstum}

Bei der Propagation werden weitere Monomereinheiten $M$ an das bei der Startreaktion gebildete Monomerradikal RI angelagert. Es kommt zur Ausbildung einer makromolekularen Kette $\mathrm{R}_{\mathrm{n}+1} \cdot$, welche eine Radikalfunktion trägt. 


$$
\mathrm{R}_{\mathrm{n}} \cdot+\mathrm{M} \stackrel{k_{\mathrm{p}}}{\longrightarrow} \mathrm{R}_{\mathrm{n}+1}
$$

\section{Gleichung 3.1.3}

Die Wachstumsgeschwindigkeit $R_{\mathrm{p}}$ wird durch folgendes Geschwindigkeitsgesetz beschrieben, wobei die Radikalkonzentration $c_{\mathrm{R}}$ und die Monomerkonzentration $c_{\mathrm{M}}$ bekannt sein muss.

$$
R_{\mathrm{p}}=-\frac{\mathrm{d} c_{\mathrm{M}}}{\mathrm{d} t}=k_{\mathrm{p}} \cdot c_{\mathrm{M}} \cdot c_{\mathrm{R}}
$$

\section{Gleichung 3.1.4}

Der Geschwindigkeitskoeffizient der Wachstumsreaktion $k_{\mathrm{p}}$ ist, wie bereits bei den Annahmen postuliert wurde, unabhängig von der Kettenlänge des Makroradikals.

\subsubsection{Kettenabbruch (Terminierung)}

Der Kettenabbruch beendet das Wachstum der Polymerkette. Diese Terminierung kann entweder durch Disproportionierung oder durch Kombination zweier Makroradikale erfolgen, wobei „totes“ Polymer entsteht, welches nicht mehr an den Elementarreaktionen beteiligt ist.

$$
\begin{aligned}
& \mathrm{R}_{\mathrm{n}} \cdot+\mathrm{R}_{\mathrm{m}} \cdot \stackrel{k_{\mathrm{t}, \mathrm{k}}}{\longrightarrow} \mathrm{P}_{\mathrm{n}+\mathrm{m}} \\
& \mathrm{R}_{\mathrm{n}} \cdot+\mathrm{R}_{\mathrm{m}} \cdot \stackrel{k_{\mathrm{t}, \mathrm{d}}}{\longrightarrow} \mathrm{P}_{\mathrm{n}}+\mathrm{P}_{\mathrm{m}}
\end{aligned}
$$

\section{Gleichung 3.1.5}

Die Kombination (Geschwindigkeitskoeffizient $k_{\mathrm{t}, \mathrm{k}}$ ) führt bei gleicher Kettenlänge der Makroradikale zu einer Verdoppelung des Polymerisationsgrades, während dieser bei Disproportionierung (Geschwindigkeitskoeffizient $k_{\mathrm{t}, \mathrm{d}}$ ) unverändert bleibt. 
Bevor die Terminierungsreaktion zweier Makroradikale stattfinden kann, muss eine räumliche Annäherung der Makroradikalketten zueinander (Translationsdiffusion) und die Umorientierung der radikalischen Kettenenden (Segmentdiffusion) vorausgehen ${ }^{[13]}$. Beide Reaktionsmöglichkeiten werden im Geschwindigkeitskoeffizienten der Terminierungsreaktion $k_{\mathrm{t}}$ berücksichtigt. Für die zeitliche Änderung der Makroradikalkonzentration $c_{\mathrm{R}}$ und damit für die Terminierungsgeschwindigkeit $R_{\mathrm{t}}$ gilt ein Geschwindigkeitsgesetz zweiter Ordnung.

$$
R_{\mathrm{t}}=-\frac{\mathrm{d} c_{\mathrm{R}}}{\mathrm{d} t}=2 \cdot k_{\mathrm{t}} \cdot c_{\mathrm{R}}^{2}
$$

\section{Gleichung 3.1.6}

\subsubsection{Kettenübertragung}

Im Verlauf der Polymerisation muss sich die Radikalfunktion nicht zwingender Weise am Kettenende eines Makroradikals befinden. Die radikalische Funktion kann auf das Monomer, den Initiator, das Polymer, das Lösungsmittel oder auf eine zugefügte Substanz übertragen werden. Die Übertragungsreaktion der radikalischen Funktion des Kettenendes auf ein anderes Molekül X erfolgt üblicherweise unter Austausch eines Wasserstoff- oder Halogenatoms.

$$
\mathrm{R}_{\mathrm{n}} \cdot+\mathrm{X} \stackrel{k_{\mathrm{tr}}}{\longrightarrow} \mathrm{P}_{\mathrm{n}}+\mathrm{X}
$$

\section{Gleichung 3.1.7}

Für diese Übertragungsreaktion kann folgendes Geschwindigkeitsgesetz angenommen werden:

$$
R_{\mathrm{tr}}=-\frac{\mathrm{d} c_{\mathrm{X}}}{\mathrm{d} t}=k_{\mathrm{tr}} \cdot c_{\mathrm{R}} \cdot c_{\mathrm{X}}
$$


Das Verhältnis des Geschwindigkeitskoeffizienten der Übertragungsreaktion $k_{\text {tr }}$ zum Geschwindigkeitskoeffizienten der Propagation $k_{\mathrm{p}}$ ist als Übertragungskonstante $C_{\mathrm{tr}}$ definiert.

$$
C_{\mathrm{tr}}=\frac{k_{\mathrm{tr}}}{k_{\mathrm{p}}}
$$

\section{Gleichung 3.1.9}

Die Radikalkonzentration bleibt im System durch die Übertragungsreaktionen unverändert und hat somit auch keinen Einfluss auf die Gesamtpolymerisationsgeschwindigkeit. Allerdings ist $\mathrm{zu}$ vermerken, dass die radikalische Funktion durch die Übertragungsreaktionen am Ende der Polymerkette verloren geht. Aus diesem Grund wird der Polymerisationsgrad eingeschränkt.

\subsection{Gesamtreaktion}

Bei einer chemisch initiierten Polymerisation wird die Radikalkonzentration als quasistationär angenommen (Bodenstein'sches Quasistationaritätsprinzip). Diese Annahme ist erfüllt, wenn die Radikalbildungsgeschwindigkeit $R_{\mathrm{d}}$ und die Kettenabbruchsgeschwindigkeit $R_{\mathrm{t}}$ identisch sind. Durch Gleichsetzen der beiden Geschwindigkeitsgesetze (3.1.2) und (3.1.6) erhält man folgenden Ausdruck:

$$
2 \cdot f \cdot k_{\mathrm{d}} \cdot c_{\mathrm{I}}=2 \cdot k_{\mathrm{t}} \cdot c_{\mathrm{R}}^{2}
$$

\section{Gleichung 3.2.1}

Der Ausdruck für die Radikalkonzentration kann nun in das Geschwindigkeitsgesetz des Kettenwachstums eingesetzt werden, wodurch man folgende Gleichung für die Polymerisationsgeschwindigkeit $R_{\mathrm{p}}$ erhält: 


$$
R_{\mathrm{p}}=-\frac{\mathrm{d} c_{\mathrm{M}}}{\mathrm{d} t}=k_{\mathrm{p}} \cdot\left(f \cdot \frac{k_{\mathrm{d}}}{k_{\mathrm{t}}}\right)^{0,5} \cdot c_{\mathrm{M}} \cdot c_{\mathrm{I}}^{0,5}
$$

Gleichung 3.2.2

\subsection{Radikalische statistische Copolymerisation}

In diesem Abschnitt werden die Grundlagen der statistischen radikalischen Copolymerisation betrachtet. Copolymere bestehen aus unterschiedlichen Monomeren. Je nach Anzahl der verwendeten Monomertypen wird eine Unterscheidung in Bi-, Ter-, Quatercopolymere usw. getroffen. Eine weitere Unterscheidungsmöglichkeit stellt die Sequenz entlang der Kette dar. Bei alternierenden Copolymeren wechseln sich die Monomertypen A und B regelmäßig ab. Statistische Copolymere besitzen eine unregelmäßige Anordnung der Monomereinheiten. Bei Gradientencopolymeren besteht schließlich ein Gradient der Copolymerzusammensetzung entlang der Kette. BlockCopolymere weisen längere Einzelsequenzen eines Monomertyps auf und PropfCopolymere besitzen an der Kette des Monomers A Seitenäste, die aus dem Monomer B aufgebaut sind. In Kapitel 3.3.1 wird das kinetische Schema einer statistischen Copolymerisation mit den Monomeren 1 und 2 erläutert. Im Kapitel 3.3.2 wird die Sequenzabhängigkeit des statistischen Copolymeren aus Monomer 1 und Monomer 2 betrachtet.

\subsubsection{Kinetik der statistischen Copolymerisation}

Die Kinetik der radikalischen Copolymerisation ist im Vergleich zur Kinetik der Homopolymerisation komplexer. Für den Fall, dass das Copolymerisationssystem nur aus zwei Monomeren besteht, müssen bereits vier Wachstumsschritte mit unterschiedlichen Geschwindigkeitskoeffizienten berücksichtigt werden. 


$$
\begin{array}{llll}
\mathrm{R}-\mathrm{M}_{1} \cdot+\mathrm{M}_{1} \stackrel{k_{11}}{\longrightarrow} \mathrm{R}-\mathrm{M}_{1}-\mathrm{M}_{1} \cdot ; & r_{11}=k_{11} \cdot c_{\mathrm{R} 1} \cdot c_{\mathrm{M} 1} \\
\mathrm{R}-\mathrm{M}_{1} \cdot+\mathrm{M}_{2} \stackrel{k_{12}}{\longrightarrow} \mathrm{R}-\mathrm{M}_{1}-\mathrm{M}_{2} \cdot ; & r_{12}=k_{12} \cdot c_{\mathrm{R} 1} \cdot c_{\mathrm{M} 2} \\
\mathrm{R}-\mathrm{M}_{2} \cdot+\mathrm{M}_{1} \stackrel{k_{21}}{\longrightarrow} \mathrm{R}-\mathrm{M}_{2}-\mathrm{M}_{1} \cdot ; & r_{21}=k_{21} \cdot c_{\mathrm{R} 2} \cdot c_{\mathrm{M} 1} \\
\mathrm{R}-\mathrm{M}_{2} \cdot+\mathrm{M}_{2} \stackrel{k_{22}}{\longrightarrow} \mathrm{R}-\mathrm{M}_{2}-\mathrm{M}_{2} \cdot ; & r_{22}=k_{22} \cdot c_{\mathrm{R} 2} \cdot c_{\mathrm{M} 2}
\end{array}
$$

\section{Abbildung 3.3.1}

Kinetisches Schema der statistischen Copolymerisation mit zwei Monomertypen.

In Abbildung 3.3.1 sind $r_{\mathrm{xx}}$ die Geschwindigkeiten der jeweiligen Wachstumsreaktion und $k_{\mathrm{xx}}$ die entsprechenden Geschwindigkeitskoeffizienten. Die Konzentrationen $c_{\mathrm{Rx}}$ und $c_{\mathrm{Mx}}$ beschreiben die entsprechenden Radikal- bzw. Monomerkonzentrationen.

In der Annahme, dass die Monomerkonzentrationen nur durch die Homo- und Kreuzwachstumsreaktionen verringert wird, gilt für den Monomerverbrauch:

$$
\frac{-\mathrm{d} c_{\mathrm{M} 1} / \mathrm{d} t}{-\mathrm{d} c_{\mathrm{M} 2} / \mathrm{d} t}=\frac{r_{11}+r_{21}}{r_{22}+r_{12}}
$$

Gleichung 3.3.1

$$
\frac{\mathrm{d} c_{\mathrm{M} 1}}{\mathrm{~d} c_{\mathrm{M} 2}}=\frac{k_{11} \cdot c_{\mathrm{R} 1} \cdot c_{\mathrm{M} 1}+k_{21} \cdot c_{\mathrm{R} 2} \cdot c_{\mathrm{M} 1}}{k_{22} \cdot c_{\mathrm{R} 2} \cdot c_{\mathrm{M} 2}+k_{12} \cdot c_{\mathrm{R} 1} \cdot c_{\mathrm{M} 2}}
$$

Gleichung 3.3.2

Mit der Bedingung des stationären Zustandes für die beiden Radikalkonzentrationen folgt, dass $r_{12}=r_{21}$ ist. In diesem Fall kann Gleichung 3.3.2 in 3.3.3 überführt werden.

$$
\frac{\mathrm{d} c_{\mathrm{M} 1}}{\mathrm{~d} c_{\mathrm{M} 2}}=\frac{1+\left(k_{11} / k_{12}\right) \cdot\left(c_{\mathrm{M} 1} / c_{\mathrm{M} 2}\right)}{1+\left(k_{22} / k_{21}\right) \cdot\left(c_{\mathrm{M} 2} / c_{\mathrm{M} 1}\right)}
$$

Gleichung 3.3.3 
Für die weiteren Erläuterungen ist es nützlich, die Verhältnisse der Geschwindigkeitskoeffizienten von Homo- zu Kreuzwachstum als Copolymerisationsparameter $r_{1}=k_{11} / k_{12}$ und $r_{2}=k_{22} / k_{21} \quad \mathrm{zu}$ definieren. Die Copolymerisationsparameter sind Verhältnisse der Geschwindigkeitskonstanten von je zwei Wachstumsreaktionen, wodurch fünf Fälle unterschieden werden können.

- $r=0 \quad$ Radikal lagert nur das „fremde“ Monomere an

- $r<1 \quad$ Radikal lagert das „fremde“ Monomere bevorzugt an

- $r=1$ gleiche Geschwindigkeitskoeffizienten für Homo- und Kreuzwachstum

- $r>1 \quad$ Radikal lagert das ,eigene“ Monomere bevorzugt an

- $r=\infty \quad$ Es erfolgt nur Homopolymerisation

Mit den Copolymerisationsparametern ergibt sich aus der Gleichung 3.3.3 die LewisMayo-Gleichung 3.3.4.

$$
\frac{\mathrm{d} c_{\mathrm{M} 1}}{\mathrm{~d} c_{\mathrm{M} 2}}=\frac{1+r_{1} \cdot\left(c_{\mathrm{M} 1} / c_{\mathrm{M} 2}\right)}{1+r_{2} \cdot\left(c_{\mathrm{M} 2} / c_{\mathrm{M} 1}\right)}
$$

\section{Gleichung 3.3.4}

Das Verhältnis der Änderung, mit denen Monomer 1 (M1) und Monomer 2 (M2) durch Kettenwachstum verbraucht werden, ist gleich dem Verhältnis der Monomerbausteine im Copolymer. Für einen differentiellen Umsatz ergibt sich eine Verknüpfung von Monomer- und Copolymerzusammensetzung.

$$
\lim _{\Delta U \rightarrow 0 \infty} \frac{\mathrm{d} c_{\mathrm{M} 1}}{\mathrm{~d} c_{\mathrm{M} 2}}=\frac{c_{\mathrm{P} 1}}{c_{\mathrm{P} 2}}
$$

\section{Gleichung 3.3.5}

Bezeichnet man das Konzentrationsverhältnis der Struktureinheiten im Copolymerisat mit $b=c_{\mathrm{P} 1} / c_{\mathrm{P} 2}$ und $a=c_{\mathrm{M} 1} / c_{\mathrm{M} 2}$ so ergibt sich Gleichung 3.3.4 zu 3.3.6. 


$$
b=\frac{1+a \cdot r_{1}}{1+r_{2} / a}
$$

\section{Gleichung 3.3.6}

Wenn man die Copolymerisationsparameter von Butadien und Styrol ${ }^{[14]}$ mit $r_{1}=1,83$ und $r_{2}=0,83$ annimmt, so entsteht folgendes Copolymerisationsdiagramm (Abbildung 3.3.2):

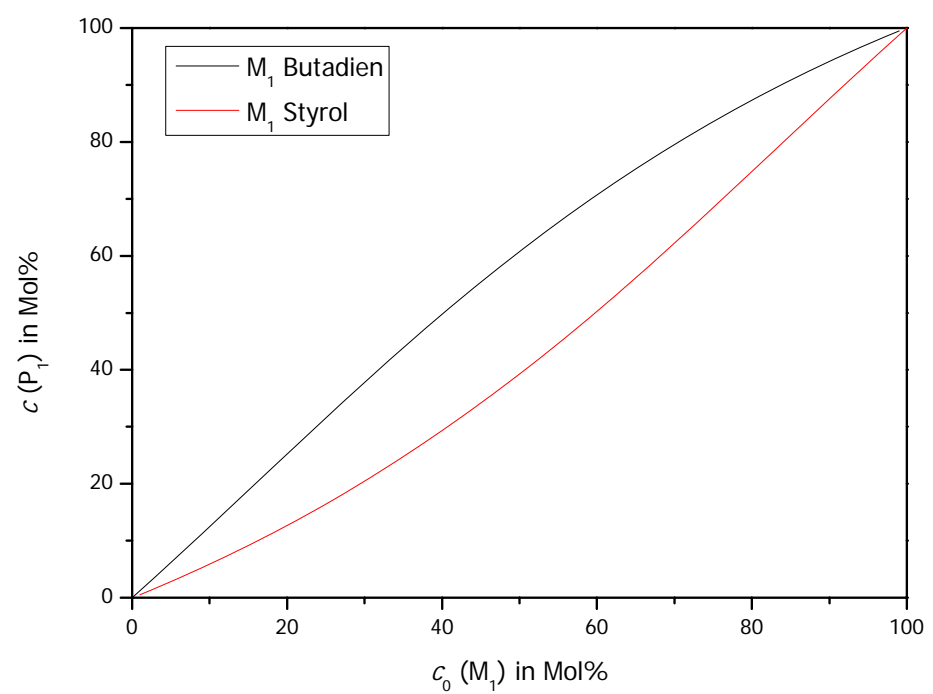

Abbildung 3.3.2

Darstellung der Copolymerzusammensetzung von 1,3-Butadien und Styrol unter der Annahme der Copolymerisationsparameter $r_{1}=1,83$ und $r_{2}=0,83[14]$.

In den Abbildungen 3.3.3 bzw. 3.3.4 werden die entsprechenden Diagramme für die Comonomere Butylacrylat bzw. Isopren in der statistischen Copolymerisaiton mit 1,3Butadien dargestellt. 


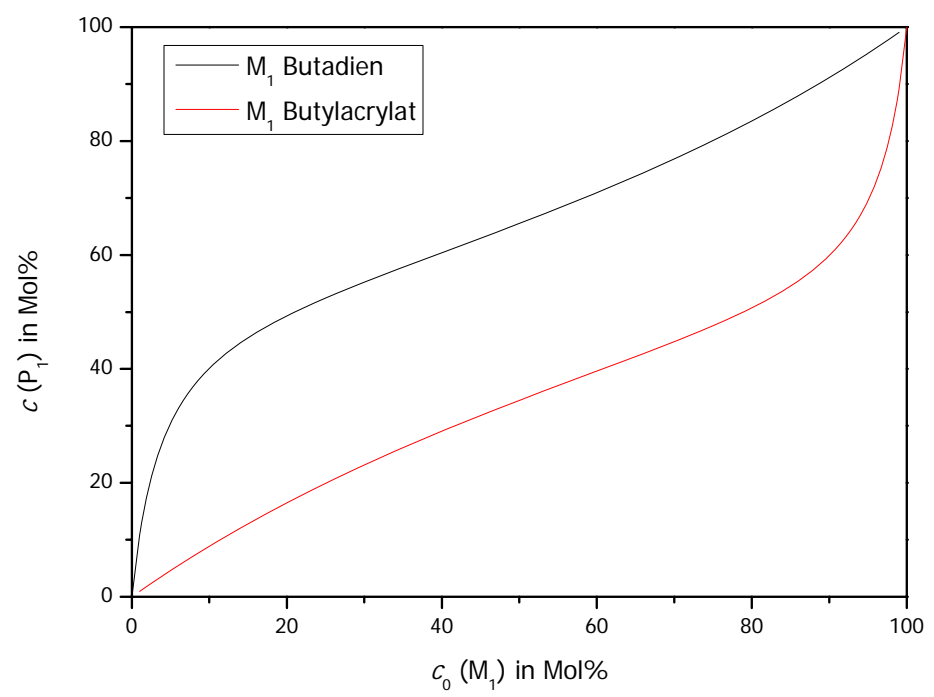

Abbildung 3.3.3

Darstellung der Copolymerzusammensetzung von 1,3-Butadien und n-Butylacrylat unter der Annahme der Copolymerisationsparameter $r_{1}=1,040$ und $r_{2}=0,074[15]$.

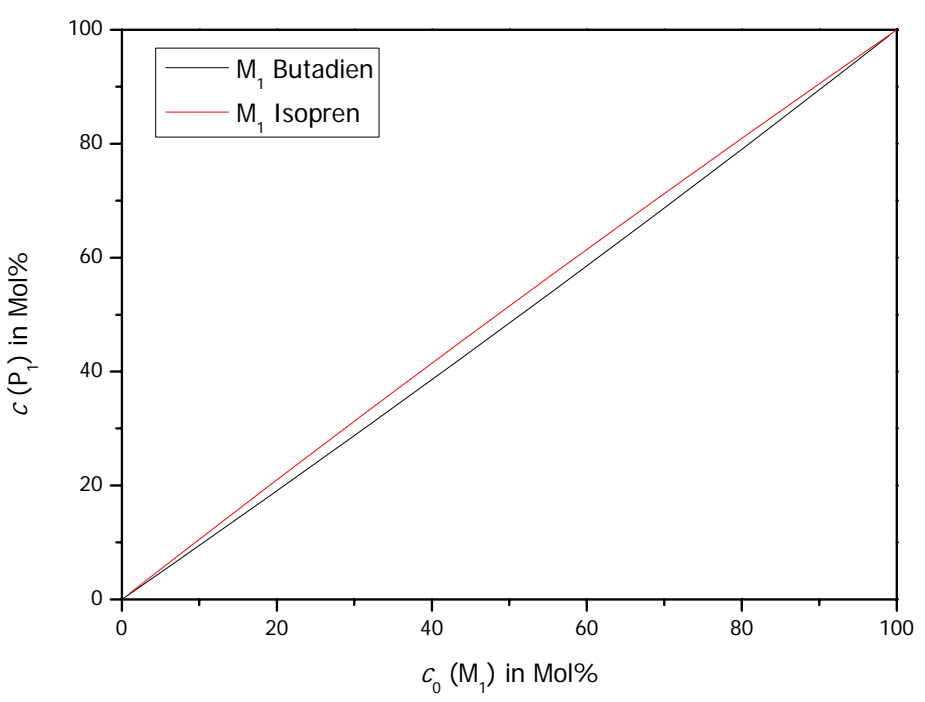

Abbildung 3.3.4

Darstellung der Copolymerzusammensetzung von 1,3-Butadien und Isopren unter der Annahme der Copolymerisationsparameter $r_{1}=0,94$ und $r_{2}=1,06^{[16]}$. 


\subsection{Grundlagen der RAFT-Polymerisation}

Im ersten Kapitel werden der Mechanismus und die Kinetik der RAFT-Polymerisation näher beschrieben. Hierbei wird der Einfluss des RAFT-Gleichgewichts auf die Kinetik der konventionellen Polymerisation näher betrachtet. Im Kapitel 3.4.2 wird die Retardierung als kinetische Besonderheit der RAFT-Polymerisation beschrieben. Im Kapitel 3.4.3 wird auf die strukturellen Unterschiede der verschiedenen RAFT-Agenzien eingegangen und die damit verbundenen kinetischen Folgen betrachtet.

\subsubsection{Mechanismus der RAFT-Polymerisation}

Mechanistisch basiert die RAFT-Polymerisation auf schwefelhaltigen Kettenübertragungsreagenzien, welche den degenerativen Kettentransfer zwischen der aktiven und der inaktiven Spezies ermöglichen ${ }^{[17-20]}$. Dabei können diese sogenannten RAFT-Agenzien in vier Hauptgruppen unterteilt werden.

Eine häufig verwendete Klasse stellen die Dithioester ${ }^{[19,21]}$ dar, welche mit einer Vielzahl von Monomeren kompatibel sind und eine gute Kontrolle der Polymerisation ermöglichen. In den letzten Jahren fanden die Trithiocarbonate ${ }^{[22-24]}$ als RAFT-Agenzien immer mehr Beachtung, da diese synthetisch einfach herzustellen sind und ebenfalls eine breite Monomerkompatibilität besitzen.

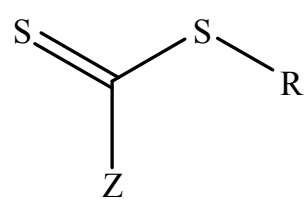

\section{Abbildung 3.4.1}

Schematische Darstellung der Grundstruktur der RAFT-Agenzien mit eine Abgangsgruppe R und einer stabilisierenden Gruppe Z.

Weiterhin sind die Xanthate als Vertreter der Dithiocarbonate ${ }^{[25]}$ zu erwähnen, welche in einem MADIX-Prozess zur Anwendung kommen. Als letzte Klasse sind zyklische oder aromatische substituierte N,N-Dithiocarbamate ${ }^{[26,27]}$ als Agenzien für RAFTPolymerisationen geeignet, obwohl sie im RAFT-Gleichgewicht eine verringerte Reaktivität aufweisen. Ein Großteil der Monomere kann mit diesen Übertragungsreagenzien kontrolliert polymerisiert werden. 
In Abbildung 3.4.1 ist die Grundstruktur eines RAFT-Agens zu sehen, wobei mit R die Abgangsgruppe und mit $Z$ die stabilisierende Gruppe bezeichnet wird.

An den strukturellen Aufbau der RAFT-Agenzien werden durch den Reaktionsmechanismus besondere Ansprüche gestellt. Eine gute Kontrolle der Polymerisation kann nur erreicht werden, wenn die am Gleichgewicht beteiligten Additions- und Fragmentierungsreaktionen schnell ablaufen. In Abbildung 3.4.2 ist das kinetische Schema der RAFT-Polymerisation beschrieben.

\section{INITIIERUNG}
I-a Initiator $\underset{f}{\stackrel{k_{\mathrm{d}}}{\longrightarrow}}$ 犃
$\mathrm{I}-\mathrm{b} \quad \stackrel{\bullet}{\stackrel{\text { Monomer }}{\longrightarrow}} \underset{k_{\mathrm{p} 1}}{\stackrel{\bullet}{*}} \mathrm{P}_{1}$

II. VOR-GLEICHGEWICHT

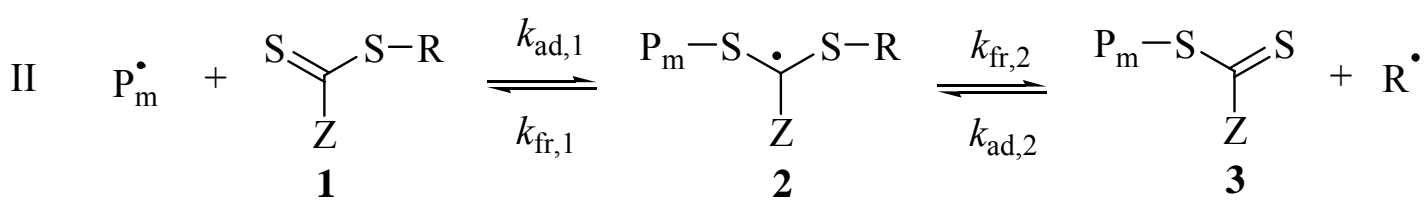

III. PROPAGATION

$$
\text { III-a } \quad \mathrm{P}_{\mathrm{n}}^{\bullet} \underset{k_{\mathrm{p}}}{\stackrel{\text { Monomer }}{\longrightarrow}} \mathrm{P}_{\mathrm{n}+1}^{\bullet} \quad \text { III-b } \quad \mathrm{R}^{\cdot} \underset{k_{\mathrm{p}, \text { rein }}}{\stackrel{\text { Monomer }}{\longrightarrow}} \mathrm{P}_{1}^{\bullet}
$$

IV. HAUPT-GLEICHGEWICHT

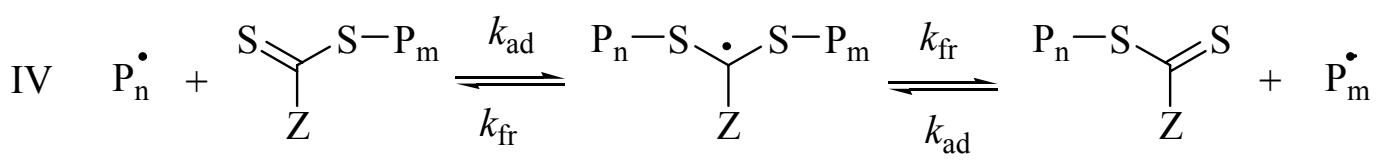

$$
\begin{aligned}
& \left(\mathrm{P}_{\mathrm{m}}-\mathrm{X}\right) \\
& \left(\mathrm{P}_{\mathrm{n}}-\mathrm{X}\right) \\
& \left(\mathrm{P}_{\mathrm{n}}-\mathrm{X}^{\cdot}-\mathrm{P}_{\mathrm{m}}\right)
\end{aligned}
$$

\section{TERMINIERUNG}

$$
\begin{array}{llll}
\mathrm{V}-\mathrm{a} & \mathrm{P}_{\mathrm{n}}^{\cdot}+\mathrm{P}_{\mathrm{m}}^{\cdot} \stackrel{<k_{\mathrm{t}}{ }^{*}(1-\mathrm{d})}{\longrightarrow} \mathrm{P}_{\mathrm{n}+\mathrm{m}} \\
\mathrm{V}-\mathrm{b} & \mathrm{P}_{\mathrm{n}}^{\cdot}+\mathrm{P}_{\mathrm{m}} \stackrel{<\stackrel{<k_{\mathrm{t}}{ }^{*} \mathrm{~d}}{\longrightarrow}}{\longrightarrow} \mathrm{P}_{\mathrm{n}}+\mathrm{P}_{\mathrm{m}}
\end{array}
$$

\section{Abbildung 3.4.2}

Darstellung eines vereinfachten kinetischen Schemas der RAFT-Polymerisation.

Nach der Initiierung durch den Zerfall des Initiators (I-a) bilden sich im ersten Reaktionsschritt Makroradikale $\mathrm{P}_{1} \cdot$ bzw. $\mathrm{P}_{\mathrm{m}} \cdot(\mathrm{I}-\mathrm{b})$. Diese Makroradikale addieren nun im 
Vorgleichgewicht (II) an das RAFT-Agens mit dem Geschwindigkeitskoeffizienten $k_{\text {ad, } 1}$ und bilden ein radikalisches Intermediat (II 2) aus. Eine sehr gute Stabilisierung des Intermediats durch die Gruppe $\mathrm{Z}$ führt $\mathrm{zu}$ einer schnelleren und effektiveren Additionsreaktion an das RAFT-Agens. Viele RAFT-Agenzien enthalten einen Phenylring als stabilisierende Gruppe. Ein zu stabiles Intermediat allerdings unterdrückt die Fragmentierungsreaktion. In diesem Fall ist der Geschwindigkeitskoeffizient $k_{\mathrm{fr}}$ stark herabsenkt und das RAFT-Gleichgewicht kann nicht schnell genug durchlaufen werden.

Dieser Effekt führt zu einer Retardierung ${ }^{[28-31]}$ der Polymerisationsgeschwindigkeit und zu einer Inhibierung. Die Stabilität des Intermediats im Vorgleichgewicht ist somit zu groß. Es entsteht ein Hybridverhalten der Polymerisation. Im Falle eines Hybridverhaltens laufen die konventionelle radikalische Polymerisation und die kontrollierte Polymerisation nebeneinander $\mathrm{ab}$, da nicht alle Kettenübertragungsreagenzien in der Anfangsphase aktiviert werden. Um die Retardierung in der Anfangsphase zu vermeiden und somit $k_{\mathrm{fr}, 2} \mathrm{zu}$ erhöhen, sollte die Abgangsgruppe ebenfalls ein stabiles Radikal ausbilden. Sterisch anspruchsvolle Gruppierungen oder ein starker elektrophiler Charakter zeichnen eine gute Abgangsgruppe aus. Im Umkehrschluss muss das Radikal R. ausreichend reaktiv sein, um seinerseits eine Reinitiierung mit Monomereinheiten durchführen zu können und somit das Makroradikal $\mathrm{P}_{\mathrm{n}} \cdot \mathrm{zu}$ bilden. Eine langsame Reinitiierung fördert Terminierungsreaktionen, welche wieder zu einer Retardierung der Polymerisationsgeschwindigkeit führen. In diesem komplexen Zusammenspiel müssen auch die sterischen und elektronischen Effekte des Monomers berücksichtigt werden. Aus diesem Grund muss eine effiziente Kombination von Abgangsgruppe und stabilisierende Gruppe für jedes neue Monomer neu gefunden werden.

Nach der Propagation (III) kann es nun zu einer reversiblen Addition des Polymerradikals $\mathrm{P}_{\mathrm{n}} \cdot$ an das polymere RAFT-Agens kommen. In diesem Fall unterscheiden sich die Abgangsgruppen nicht mehr voneinander und das RAFT-Hauptgleichgewicht (IV) wird wirksam. Im Hauptgleichgewicht (IV) addiert ein wachsenden Radikal $\mathrm{P}_{\mathrm{n}} \cdot$ reversibel an ein polymeres RAFT-Agens. Es erfolgt die Freisetzung des Polymerradikals $\mathrm{P}_{\mathrm{m}}$. als aktive Spezies, welches nun durch Anlagerung von weiteren Monomereinheiten propagieren kann. Aufgrund der Geschwindigkeit der Additions- und Fragmentierungsreaktion sind meist alle wachsenden Ketten an einem RAFT-Gleichgewicht beteiligt und liegen nicht lange in freier Form vor. Ein schneller Wechsel zwischen Additions- und Fragmentierungsreaktionen und somit ein „lebendes“ Gleichgewicht garantieren ein 
simultanes Wachstum aller Polymerketten und eine enge Molekulargewichtsverteilung. Am Ende der Polymerisation besitzen fast alle Polymerketten eine Endgruppe, welche dem entsprechenden RAFT-Agens entspricht. Diese können im Sinne der „lebenden“ Polymerisation reaktiviert werden und wieder neue Monomereinheiten addieren, um z.B. Blockcopolymere herzustellen.

Die Kettenlänge oder der Polymerisationsgrad lassen sich unter Berücksichtigung der eingesetzten RAFT-Agens-Konzentration $c_{0}$ (RAFT), der Monomerkonzentration $c_{0}$ (Monomer) und der partiellen Umsätze $U_{\mathrm{P}}$ voraussagen.

$$
P_{\mathrm{n}}=\frac{c_{0}(\text { Monomer })}{c_{0}(\mathrm{RAFT})} \cdot U_{\mathrm{P}}
$$

\section{Gleichung 3.4.1}

Weiterhin kann mit Hilfe des Polymerisationsgrads auch die Polydispersität (PDI) oder die Breite der Molekulargewichtsverteilung beschrieben werden. Die Polydispersität strebt bei einer idealen RAFT-Polymerisation nach Gleichung 3.4.2 mit zunehmendem Polymerisationsgrad gegen eins.

$$
P D I=\frac{M_{\mathrm{w}}}{M_{\mathrm{n}}}=1+\frac{1}{\overline{P_{\mathrm{n}}}}
$$

\section{Gleichung 3.4.2}

Die mittleren Molekulargewichte $\bar{M}_{\mathrm{n}}, \bar{M}_{\mathrm{w}}$ und $\bar{M}_{\mathrm{n}}^{\text {theo }}$ werden im Rahmen dieser Arbeit als $M_{\mathrm{n}}, M_{\mathrm{w}}$ und $M_{\mathrm{n}}^{\text {theo }}$ bezeichnet. Ebenso werden die Zahlen- und Gewichtsmittelwerte der Kettenlänge $\overline{P_{\mathrm{n}}}$ und $\overline{P_{\mathrm{w}}}$ als $P_{\mathrm{n}}$ und $P_{\mathrm{w}}$ beschrieben. 


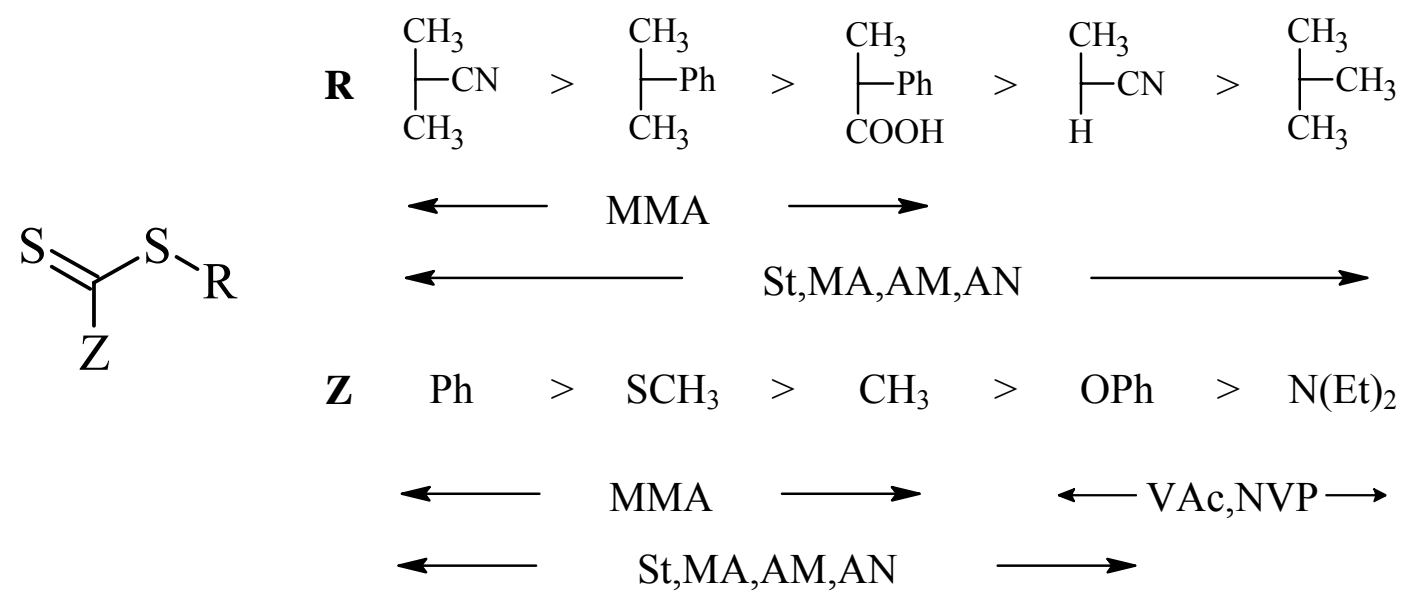

Abbildung 3.4.3

Schematischer Überblick über den strukturellen Aufbau von Thiocarbonylthioverbindungen, welche als RAFT-Agenzien für verschiedene Monomere fungieren können (St $=$ Styrol, $M M A=$ Methylmethacrylat,$\quad M A=$ Methacrylat,$\quad V A c=$ Vinylacetat,$\quad A M=$ Acrylamid, $A N=$ Acrylnitril, $N V P=N$-Vinylpyrrolidon, $N V C=N$-Vinylcarbazol). Für die R-Gruppe nimmt die Fragmentierungsrate von links nach rechts ab. Für die Z-Gruppe nimmt die Fähigkeit der Addition von Radikalfunktionalitäten von links nach rechts ab, wobei die Fragmentierungsrate des Intermediats zunimmt.

\subsubsection{Retardierung der Polymerisationsgeschwindigkeit}

Im Idealfall der RAFT-Polymerisation wird die Kinetik der konventionellen radikalischen Polymerisation nur durch das Vor- bzw. Hauptgleichgewicht überlagert. Die effektive Radikalkonzentration wird also im direkten Vergleich nicht verändert und die Geschwindigkeiten der Terminierung und der Propagation sind identisch. Allerdings wird bei höheren RAFT-Agens-Konzentrationen eine Abnahme der Polymerisationsgeschwindigkeit beobachtet. Dieser Effekt wird als Retardierung ${ }^{[32,33]}$ bezeichnet und hängt mit der Struktur des RAFT-Agens zusammen. In der Literatur konnte noch nicht eindeutig geklärt werden, welche Gründe für die Retardierung einiger RAFT-Agenzien verantwortlich sind. Sicher scheint nur, dass das Intermediat im RAFTGleichgewicht eine entscheidende Rolle spielt und an Nebenreaktionen beteiligt ist. Der Retardierungseffekt nimmt mit steigender Stabilität des radikalischen Intermediats zu. Folglich muss also die effektive Radikalkonzentration im Vergleich zur konventionellen radikalischen Polymerisation reduziert werden. Folgende Szenarien sind hierfür denkbar: 


$$
\begin{aligned}
& \mathrm{P}_{\mathrm{m}}-\mathrm{S} \frac{\stackrel{\mathrm{R}}{\mathrm{Z}} \mathrm{S}}{\mathrm{Z}}-\mathrm{P}_{\mathrm{n}} \\
& <k_{\mathrm{t}, \mathrm{int}-\mathrm{R}}>\mid \\
& \text { Radikal } \\
& +
\end{aligned}
$$

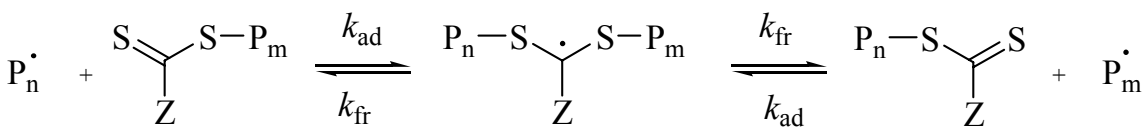

$$
\begin{aligned}
& \mid<k_{\mathrm{t}, \text { int-int }}> \\
& { }_{\mathrm{P}_{\mathrm{m}}-\mathrm{S}}^{\mathrm{P}_{\mathrm{n}}-\mathrm{S}}=\longrightarrow \longrightarrow \mathrm{S}_{\mathrm{S}-\mathrm{P}_{\mathrm{m}}}^{\mathrm{S}-\mathrm{P}_{\mathrm{n}}} \\
& \text { mögliches Terminierungsprodukt }
\end{aligned}
$$

\section{Abbildung 3.4.4}

Schematische Übersicht über mögliche Reaktionswege, welche die effektive Radikalkonzentration während der RAFT-Polymerisation erniedrigen und somit die Retardierung bewirken.

In Abbildung 3.4.4 sind die möglichen Reaktionswege des Intermediats aufgezeichnet. Nach der Addition eines Makroradikals kann das Intermediat mit sich selbst oder mit einem anderen Makroradikal terminieren ${ }^{[28]}$. In beiden Fällen verläuft der Terminierungsschritt irreversibel. Durch diese irreversible Terminierung wird die Gesamtkonzentration an Radikalen reduziert und somit die Polymerisationsgeschwindigkeit erniedrigt ${ }^{[31,} 34,{ }^{35}$. In jedem Fall wird die Radikalfunktionalität gespeichert und steht der Kinetik der Polymerisation nicht mehr zur Verfügung. Bei beiden Varianten müssten als Terminierungsprodukt Vierarm- bzw. Dreiarmsterne entstehen, welche bisher allerdings experimentell noch nicht bestätigt werden konnten. Aus diesem Grund wurde von Buback et al. ein Mechanismus vorgeschlagen $^{[36]}$, bei dem ein wachsendes Makroradikal mit einen Dreiarmstern zu einer toten Polymerkette reagiert und das RAFT-Intermediat zurückgebildet wird. Theoretische Berechnungen ${ }^{[37]}$ untermauern die vorgeschlagene Rückgewinnung des RAFT-Agens. 


\subsection{Molekulargewichtsverteilung}

Die Bestimmung der Molekulargewichtsverteilung ist eine essentielle Analysemethode in der Polymerchemie, da diese ein wichtiges Charakteristikum von Polymeren ist. In der Molekulargewichtsverteilung spiegelt sich vor allem die Kinetik der Polymerisation wider und es können somit Rückschlüsse auf die Geschwindigkeitskoeffizienten der Elementarreaktionen (Kapitel 3.1) gezogen werden. Als Beispiel sind hier die von Olaj et al. entwickelten PLP-SEC-Experimente ${ }^{[38]} \mathrm{zu}$ nennen. In Laser-induzierten Polymerisationen werden dabei Polymere hergestellt, aus deren Molekulargewichtsverteilungen der Wachstumsgeschwindigkeitskoeffizient $k_{\mathrm{p}}$ bestimmt werden kann. Messungen von Molekulargewichtsverteilungen werden mit Hilfe der Gel-Permeations-Chromatographie durchgeführt. Üblicherweise werden Molekulargewichtsverteilungen in der differentiellen Form dargestellt, obwohl auch Verteilungen in der integralen Form vorkommen. Bei einer differentiellen Verteilung werden die Massenanteile $w_{\mathrm{i}}$ einer bestimmten Kettenlänge gegen die entsprechenden Molekulargewichte $M_{\mathrm{i}}$ aufgetragen. Der Zahlenmittelwert des Molekulargewichts und der Gewichtsmittelwert des Molekulargewichts sind wichtige Kenngrößen einer Molekulargewichtsverteilung. Diese Größen werden im Rahmen dieser Arbeit vereinfacht als $M_{\mathrm{n}}$ und $M_{\mathrm{w}}$ bezeichnet.

$$
M_{\mathrm{n}}=\frac{\sum_{i=1}^{\infty} N_{\mathrm{i}} \cdot M_{\mathrm{i}}}{\sum_{i=1}^{\infty} \cdot N_{\mathrm{i}}}
$$

\section{Gleichung 3.5.1}

$N_{\mathrm{i}}=$ Anzahl der Makromoleküle mit dem Molekulargewicht $M_{\mathrm{i}}$ 


$$
M_{\mathrm{w}}=\frac{\sum_{i=1}^{\infty} m_{\mathrm{i}} \cdot M_{\mathrm{i}}}{\sum_{i=1}^{\infty} m_{\mathrm{i}}}=\sum_{i=1}^{\infty} w_{\mathrm{i}} \cdot M_{\mathrm{i}}
$$

\section{Gleichung 3.5.2}

$m_{\mathrm{i}}=$ Gesamtmasse aller Makromoleküle mit dem Molekulargewicht $M_{\mathrm{i}}$

$w_{\mathrm{i}}=$ Massenanteil der Makromoleküle mit dem Molekulargewicht $M_{\mathrm{i}}$ an der Gesamtmasse

Diese Kenngrößen charakterisieren die Breite der Verteilung, welche auch als Polydispersitätsindex (PDI) bezeichnet wird.

$$
P D I=\frac{M_{\mathrm{w}}}{M_{\mathrm{n}}}
$$

\section{Gleichung 3.5.3}

Mit Hilfe dieser Kenngrößen lassen sich Molekulargewichtsverteilungen von Polymerproben miteinander vergleichen.

Im Prinzip lassen sich statistische Kennzahlen einer Molekulargewichtsverteilung $f\left(M_{\mathrm{i}}\right)$ auch als einzelne Momente der Verteilung ausdrücken.

In einer Verteilung ist das $k$-te Moment folgendermaßen definiert:

$$
\mu^{k}=\sum_{i=1}^{\infty} f\left(M_{i}\right) \cdot M_{i}^{k}
$$

\section{Gleichung 3.5.4}

Der Zahlen- und Gewichtsmittelwert des Molekulargewichts ergeben sich nun aus den Quotienten bestimmter Momente. Für den Zahlenmittelwert des Molekulargewichts $M_{\mathrm{n}}$ kann die Gleichung 3.5.5 angenommen werden. 


$$
M_{\mathrm{n}}=\frac{\mu^{1}}{\mu^{0}}=\frac{\sum_{i=1}^{\infty} x_{\mathrm{i}} \cdot M_{\mathrm{i}}}{\sum_{i=1}^{\infty} x_{\mathrm{i}}}=\sum_{i=1}^{\infty} x_{\mathrm{i}} \cdot M_{\mathrm{i}}
$$

\section{Gleichung 3.5.5}

$x_{\mathrm{i}}=$ Zahlenverteilung über die Molekulargewichte $M_{\mathrm{i}}$

Der Gewichtsmittelwert des Molekulargewichts $M_{\mathrm{w}}$ ist wie folgt definiert:

$$
M_{\mathrm{w}}=\frac{\mu^{2}}{\mu^{1}}=\frac{\sum_{i=1}^{\infty} x_{\mathrm{i}} \cdot M_{\mathrm{i}}^{2}}{\sum_{i=1}^{\infty} x_{\mathrm{i}} \cdot M_{\mathrm{i}}}
$$

\section{Gleichung 3.5.6}

Im folgenden Abschnitt sollen noch einmal die Auswirkungen der Kinetik auf die Molekularverteilung der konventionellen radikalischen Polymerisation verdeutlicht werden. Es ist möglich aus den kinetischen Parametern die Kettenlängenverteilungen und damit die Molekulargewichtsverteilung theoretisch zu bestimmen.

Für diese Berechnungen wird eine Wachstumswahrscheinlichkeit $\alpha$ definiert. $\alpha$ beschreibt die Wahrscheinlichkeit, ob ein gebildetes Radikal im nächsten Schritt wächst oder terminiert.

Die Geschwindigkeiten der einzelnen Elementarreaktionen einer konventionellen radikalischen Polymerisation definieren die Wachstumswahrscheinlichkeit $\alpha^{[12]}$ folgendermaßen:

$$
\alpha=\frac{R_{\mathrm{p}}}{R_{\mathrm{p}}+R_{\mathrm{tr}}+R_{\mathrm{t}}}
$$

\section{Gleichung 3.5.7}


Falls die Propagation gegenüber der Terminierung und dem Kettentransfer stark bevorzugt wird, so ist die Wachstumswahrscheinlichkeit $\alpha \approx 1$.

Die Wachstumsgeschwindigkeit kann allerdings nur Aussagen über die Molekulargewichtsverteilung prognostizieren, wenn zwischen Kombination und Disproportionierung bei den Möglichkeiten der Terminierung unterschieden wird.

Zunächst wird die Disproportionierung als Terminierungsreaktion angenommen. Die Wahrscheinlichkeit $x_{\mathrm{P}, \mathrm{d}}$, dass im Fall der Disproportionierung eine Kette mit der Kettenlänge $\mathrm{P}$ auftritt, ergibt sich aus folgender Gleichung:

$$
x_{P, \mathrm{~d}}=\alpha^{P-1} \cdot(1-\alpha)
$$

\section{Gleichung 3.5.8}

Hierbei wird berücksichtigt, dass die Kette (P-1) mal wächst $\left(\alpha^{\mathrm{P}-1}\right)$ und schließlich einmal terminiert $(1-\alpha)$.

Die Kettenlängen- und Molekulargewichtsverteilungen variieren um ein Vielfaches des Molekulargewichtes einer Monomereinheit. Aus diesem Grund können die charakteristischen Kennzahlen einer Verteilung auch hier aus den einzelnen Momenten bestimmt werden. Mit Hilfe der Kettenlänge $P$ und der Kettenlängenwahrscheinlichkeit $x_{\mathrm{P}, \mathrm{d}}$ sind die Momente $\mu^{\mathrm{k}}$ (analog zu Gleichung 3.5.4) folgendermaßen definiert:

$$
\mu^{\mathrm{k}}=\sum_{P=1}^{\infty} x_{P, \mathrm{~d}} \cdot P^{\mathrm{k}}
$$

Gleichung 3.5.9 


\section{Theoretische Grundlagen}

Für die Zahlen- und Gewichtsmittelwerte der Kettenlänge ergeben sich folgende Ausdrücke:

$$
P_{\mathrm{n}, \mathrm{d}}=\frac{\mu^{1}}{\mu^{0}}=\frac{(1-\alpha)^{-1}}{1}=(1-\alpha)^{-1}
$$

Gleichung 3.5.10

$$
P_{\mathrm{w}, \mathrm{d}}=\frac{\mu^{2}}{\mu^{1}}=\frac{(1+\alpha) \cdot(1-\alpha)^{-2}}{(1-\alpha)^{-1}}=\frac{(1+\alpha)}{(1-\alpha)}
$$

Gleichung 3.5.11

Mit dem Zahlen- und Gewichtsmittelwert der Kettenlänge kann nun der Polydispersitätsindex definiert werden.

$$
P D I=\frac{P_{\mathrm{w}, \mathrm{d}}}{P_{\mathrm{n}, \mathrm{d}}}=1+\alpha
$$

\section{Gleichung 3.5.12}

Im Falle einer bevorzugten Propagation ergibt sich für die Disproportionierung ein Polydispersitätsindex von ungefähr $P D I=2$.

Für die Kettenlängenwahrscheinlichkeit $x_{\mathrm{P}, \mathrm{c}}$ unter Berücksichtigung der Kombination zweier Radikale mit den Kettenlängen $\mathrm{m}$ und $\mathrm{n}$ gilt folgender Ausdruck $(\mathrm{P}=\mathrm{n}+\mathrm{m})$ :

$$
x_{P, \mathrm{c}}=\sum_{n=1}^{P-1} \alpha^{n-1} \cdot(1-\alpha) \cdot \alpha^{m-1} \cdot(1-\alpha)=(P-1) \cdot \alpha^{P-2}(1-\alpha)^{2}
$$

\section{Gleichung 3.5.13}


Es wird angenommen, dass zwei Makroradikalketten $n$ - bzw. $m$-mal gewachsen sind $\left(\alpha^{\mathrm{n}-1} / \alpha^{\mathrm{m}-1}\right)$ und jeweils nur einmal terminieren (1- $\left.\alpha\right)$. Um einer Gesamtkettenlänge $P$ der Kombination zu erhalten, erfolgt eine Summation der Makroradikalkettenlängen. Für die Zahlen- und Gewichtsmittelwerte der Kettenlänge ergeben sich folgende Ausdrücke:

$$
P_{\mathrm{n}, \mathrm{c}}=\frac{\mu^{1}}{\mu^{0}}=\frac{2 \cdot(1-\alpha)^{-1}}{1}=2 \cdot(1-\alpha)^{-1}
$$

Gleichung 3.5.14

$$
P_{\mathrm{w}, \mathrm{c}}=\frac{\mu^{2}}{\mu^{1}}=\frac{(4+2 \alpha) \cdot(1-\alpha)^{-2}}{2(1-\alpha)^{-1}}=(2+\alpha)(1-\alpha)^{-1}
$$

\section{Gleichung 3.5.15}

Analog kann nun der Polydispersitätsindex bestimmt werden:

$$
P D I=\frac{P_{\mathrm{w}, \mathrm{d}}}{P_{\mathrm{n}, \mathrm{d}}}=1+\frac{\alpha}{2}
$$

\section{Gleichung 3.5.16}

Mit der Annahme $\alpha=1$ ergibt sich somit ein Polydispersitätsindex von $P D I=1,5$. Es wird nun deutlich, dass Produkte einer konventionellen radikalischen Polymerisation einen Polydispersitätsindex von $P D I=1,5$ nicht unterschreiten können.

Für kontrollierte Polymerisationen ist die Ableitung der Kettenlängenverteilung aus der Wachstumswahrscheinlichkeit $\alpha$ nicht mehr so einfach möglich. Die Kettenlängenverteilungen von idealen lebenden Polymerisationen lassen sich allerdings gut durch die Poisson-Verteilung beschreiben. In dieser Verteilung wird berücksichtigt, dass alle Ketten simultan starten und in gleicher Art und Weise wachsen. Diese Annahmen führen nicht automatisch $\mathrm{zu}$ einheitlichen Kettenlängen, da der 


\section{Theoretische Grundlagen}

Wachstumsschritt einer statistischen Streuung unterworfen ist. Für die Wahrscheinlichkeit $x_{P}$, dass eine Kette mit der Kettenlänge $P$ auftritt, kann angenommen werden:

$$
x_{P}=\frac{v^{P} \cdot e^{-v}}{P !}
$$

\section{Gleichung 3.5.17}

Wahrscheinlichkeit $x_{p}$ für das Auftreten einer Ketten mit der Kettenlänge $P$ unter Berücksichtigung des Erwartungswertes der kinetischen Kettenlänge $v$.

Die Poisson-Verteilung wird ebenfalls durch ihre Momente beschrieben, aus denen sich der Zahlen- und Gewichtsmittelwert der Kettenlänge P berechnen lassen:

$$
P_{\mathrm{n}}=\frac{\mu^{1}}{\mu^{0}}=\frac{v}{1}=v
$$

\section{Gleichung 3.5.18}

$$
P_{\mathrm{w}}=\frac{\mu^{2}}{\mu^{1}}=\frac{v^{2}+v}{v}=v+1
$$

\section{Gleichung 3.5.19}

Der Polydispersitätsindex ergibt sich nun aus dem Zahlen- und Gewichtsmittelwert der Kettenlänge $P$.

$$
P D I=\frac{P_{\mathrm{w}}}{P_{\mathrm{n}}}=\frac{v+1}{v}=1+\frac{1}{v}=1+\frac{1}{P_{\mathrm{n}}}
$$

\section{Gleichung 3.5.20}

Im Gegensatz zur konventionellen radikalischen Polymerisation nimmt der Polydispersitätsindex einer lebenden Polymerisation mit zunehmender mittlerer Kettenlänge $a b$ und nähert sich dem Grenzwert von eins. 
Die Poisson-Verteilung ist eine ein parametrige, diskrete Verteilung. Der Zahlenmittelwert der Kettenlänge und die Breite der Verteilung bzw. die Varianz $\sigma^{2}$ sind identisch mit dem Erwartungswert $v$. Somit werden durch einen Parameter die Lage und die Breite der Verteilung festgelegt.

Anzumerken ist schließlich, dass für große Kettenlängen $P$ die Poisson-Verteilung in eine Gauß-Verteilung übergeht, die durch Gleichung 3.5.21 beschrieben wird.

$$
x_{\mathrm{i}}=\frac{1}{2 \pi \cdot v} \cdot \exp \left(\frac{(P-v)^{2}}{2 v}\right)
$$

\section{Gleichung 3.5.21}

\subsection{Berechnung von theoretischen Molekulargewichten}

Im Rahmen der Auswertung der einzelnen Polymerisationen werden auch immer wieder die experimentell ermittelten Werte für die Molekulargewichte (Molmassen) mit den theoretischen Werten verglichen, welche sich aus der Kinetik der RAFT-Polymerisation im idealen Fall ergeben. Es gibt verschiedene Möglichkeiten die theoretischen Molmassen zu bestimmen. In Gleichung 3.6.1 wird der einfachste Fall angenommen. Hierbei ist $U$ der Umsatz, $M$ (Monomer) bezeichnet die Molmasse des Monomers, $c_{0}$ (Monomer) ist die Ausgangskonzentration an Monomer und $c_{0}$ (RAFT) ist die ursprüngliche Konzentration des RAFT-Agens. Die Molmasse des Polymers beginnt mit der Molmasse des RAFTAgens $M($ RAFT). Danach gibt es eine stetige lineare Zunahme der Molmasse mit dem Umsatz $(U)$.

$$
M_{n}^{\text {theo }}=\frac{U \cdot c_{0}(\text { Monomer }) \cdot M(\text { Monomer })}{c_{0}(\mathrm{RAFT})}+M(\mathrm{RAFT})
$$

Gleichung 3.6.1 


$$
M_{n}^{\text {theo }}(\mathrm{T})=\frac{U \cdot c_{0}(\text { Monomer }) \cdot M(\text { Monomer })}{c_{0}(\mathrm{RAFT})+c_{0}(\text { Initiatior }) \cdot f \cdot d \cdot\left(1-e^{-k_{d} \cdot t}\right)}+M(\mathrm{RAFT})
$$

\section{Gleichung 3.6.2}

Gleichung 3.6.2 basiert auf der Gleichung 3.6.1, ist allerdings um den Effekt der Terminierung erweitert worden. Jeder Zerfall des Initiators bildet neue Radikale aus, die die Polymerisation vorantreiben aber auch vorhandene Polymerradikale irreversibel terminieren. Dieser Effekt lässt die mittlere Molmasse bei höheren Reaktionszeiten nicht mehr so schnell anwachsen, wie durch $M_{\mathrm{n}}^{\text {theo }}$ vorhergesagt. Der Koeffizient $f$ beschreibt dabei die Effektivität des Initiators und kann üblicherweise einen Wert zwischen 0,6 und 1,0 annehmen. Für die Berechnungen in dieser Arbeit wird für $f$ ein Wert von $0,8^{[39]}$ angenommen. Die Anzahl der Ketten, die bei der Terminierung entstehen, werden durch den Faktor $d$ wiedergegeben und können abhängig vom Terminationsmodus Werte zwischen 1,0 und 2,0 annehmen. Da die Polybutadienradikale anscheinend bevorzugt über den Kombinationsmechanismus terminieren ${ }^{[39]}$, wird für $d$ ein Wert von 1,20 angenommen. Weiterhin wird in dieser Gleichung die Ausgangskonzentration $c_{0}$ (Initiator) und die Zerfallskonstante des Initiators $k_{\mathrm{d}}$ berücksichtigt. Bei geringen Umsätzen sind die Werte für die beiden Gleichungen annähernd gleich. Im Verlauf der Polymerisation allerdings wachsen die Werte für $M_{\mathrm{n}}^{\text {theo }}(\mathrm{T})$ nicht mehr so schnell an, wodurch der reale Verlauf der Molmassen in RAFT-Polymerisationen besser beschrieben wird. Im Rahmen der Auswertung werden GPC-Kurven von statistischen Copolymeren gemessen. Eine Molmassenbestimmung mittels GPC erfolgt über eine Eichung für das entsprechende Polymer. Aus diesem Grund können verschiedene Messungen mit unterschiedlichen Eichungen durchgeführt werden. In diesem Fall werden natürlich unterschiedliche Werte für die mittleren Molmassen für ein und dasselbe Polymer bestimmt. Demnach können auch für die theoretischen Molmassen zwei Werte berechnet werden, indem die Molmasse des Monomers verändert wird. 


\section{Experimenteller Aufbau I}

In diesem Kapitel wird der in dieser Arbeit entwickelte, experimentelle Aufbau für die Polymerisation von gasförmigen Monomeren näher beschrieben. Im ersten Unterkapitel werden die Anforderungen, welche an das experimentelle Setup gestellt werden, formuliert und die Besonderheiten im Umgang mit 1,3-Butadien erläutert. Im Laufe dieser Arbeit wurden zwei experimentelle Aufbauten realisiert. Der „kleine“ Autoklav (Aufbau I) eignet sich für niederviskose Polymerisationsansätze und wird im Kapitel 4.2 im Detail beschrieben. Auf der Grundlage der Erfahrungen im Umgang mit dem „kleinen“ Autoklaven wurde für die Polymerisation in heterogenen Polymerisationssystemen und für die Herstellung von Polymerproben mit einer Masse größer als $35 \mathrm{~g}$ ein neuer experimenteller Aufbau II entwickelt. Im Kapitel 5 wird auf diese Neuentwicklung eingegangen.

\subsection{Anforderungen an den experimentellen Aufbau I}

Der Umgang mit gasförmigen Monomeren erschwert den normalen Polymerisationsablauf enorm. Alleine das Einbringen einer definierten Menge einer gasförmigen Substanz in ein Polymerisationssystem ist nur mit erhöhtem Aufwand realisierbar. Neben der Dichtigkeit und der Druckbeständigkeit sollte die Apparatur natürlich ein sicheres und reproduzierbares Arbeiten ermöglichen. Der Sicherheitsaspekt ist aufgrund der toxikologischen Eigenschaften von 1,3-Butadien besonders zu betonen. Eine Möglichkeit der fachgerechten Entsorgung von Restmonomer nach der Polymerisation muss ebenso in die Apparatur integriert werden, wie die Möglichkeit der sicheren Probenentnahme während der Polymerisation. Im Speziellen lassen sich die geforderten Grundparameter des experimentellen Aufbaus wie folgt zusammenfassen:

Die Polymerisationen sollen in einem Temperaturbereich von Raumtemperatur bis $120^{\circ} \mathrm{C}$, sowie in einem Druckbereich bis maximal 50 bar, durchgeführt werden. Das Temperaturintervall ist geeignet für eine RAFT-Polymerisation von vinylischen Monomeren und der Maximaldruck ergibt sich aus der thermischen Ausdehnung von 1,3Butadien selbst, wobei hier ein gewisser Sicherheitsbereich einkalkuliert ist. Aufgrund der thermischen Initiierung sind eine hohe Aufheizrate sowie eine sensible Temperaturregelung unerlässlich. Die gewünschte Reaktionstemperatur soll schnell und ohne Überhitzungs- oder Unterkühlungsphasen erreicht werden, um eine Reproduzierbarkeit der Ergebnisse zu ermöglichen. Des Weiteren muss das mehrfache 
Befüllen mit definierten Mengen an 1,3-Butadien ermöglicht werden. Nur so lassen sich die Monomerumsätze exakt bestimmen und das Polymerisationssystem näher untersuchen. Weiterhin sollte eine gute Durchmischung der Reaktionslösung gegeben sein, um die Homogenität des Systems zu garantieren und somit Konzentrationsgefällen entgegen zu wirken. Natürlich sollte die Rührleistung auch für hochviskose Lösungen geeignet sein, um die Polymerisation in Gegenwart von Füllstoffen wie Silica oder Ruß zu ermöglichen. Um die störenden Einflüsse von Sauerstoff in der Polymerisationslösung zu minimieren, sollte die komplette Apparatur mit Stickstoff gespült werden können. Eine weitere Anforderung stellt die Probenentnahme während einer Polymerisation dar. Die Fähigkeit, Proben nach verschiedenen Zeiten bzw. Umsätzen zu ziehen, ist unerlässlich für die Bewertung und Beschreibung eines kontrollierten radikalischen Polymerisationsprozesses. Schließlich wird auf die leichte Handhabung und Reinigung des apparativen Aufbaus großen Wert gelegt, um einen großen Probendurchsatz zu gewährleisten bzw. um Verschmutzungen und andere Störeffekte auszuschließen.

All diese Anforderungen konnten in unterschiedlichen Ausmaßen in den beiden experimentellen Aufbauten I und II realisiert werden.

\subsection{Hochdruck - Laborautoklav I „Modell II“}

Im folgenden Kapitel soll auf den apparativen Aufbau des „kleinen“ Autoklaven eingegangen werden. Es wird die erstmalige Montage und die Erweiterung mit Sonderzubehör beschrieben.

\subsubsection{Technische Daten und Beschreibung des Aufbaus}

Die Polymerisation von 1,3-Butadien wird in einem Hochdruck-Laborautoklav „Modell II“ der Firma Carl Roth ${ }^{\circledR}$ durchgeführt. Der Versuchsaufbau besteht aus V4A-Edelstahl und ist für alle Reaktionen, bei denen ein rascher Druckanstieg zu erwarten ist, geeignet. Der Versuchsautoklav lässt sich in drei Hauptbestandteile unterteilen. Er besteht aus dem zylindrischen Autoklavenbecher, dem Autoklavenkopf und dem Zwischenstück mit Tauchrohr. Der Autoklavenkopf besitzt drei seitliche $1 / 4$ " G Gewindeöffnungen, die für die Aufnahme des Zubehörs (Manometer, Ventile) geeignet sind. Der gesamte Reaktionsaufbau ist auf einen maximalen Arbeitsdruck von 100 bar ausgelegt. Die Abdichtung des Autoklaven erfolgt über Flachdichtungen aus Polytetrafluorethylen (PTFE), welche bis zu einer Arbeitstemperatur von $180^{\circ} \mathrm{C}$ eingesetzt werden können. Die 


\section{Experimenteller Aufbau I}

Verschraubungen, bei denen die Abdichtung mit PTFE-Dichtungen erfolgt, werden mit dem passenden Schraubenschlüssel nur handfest angezogen, um einen unnötigen Verschleiß der Dichtungen zu verhindern. Bei der Verwendung von Feinsilberdichtungen werden die Verschraubungen fest angezogen. Alle Gewinde sind mit Teflonspray einzusprühen, um ein Kaltverschweißen der Edelstahlkomponenten zu unterdrücken und einen materialschonenden Umgang zu garantieren.

Als erstes wird das Zwischenstück mit dem Tauchrohr auf den Autoklavenkopf gesetzt. Hierzu wird die PTFE-Dichtung ( $\varnothing$ außen $42 \mathrm{~mm}$ ) in die obere Kopföffnung eingelegt und dann das Zwischenstück eingeschraubt und mit einem Schlüssel der Größe 55 leicht angezogen. Am Zwischenstück befindet sich eine seitliche $1 / 4^{\prime \prime G ~ G e w i n d e o ̈ f f n u n g, ~ i n ~ d i e ~}$ das Reduzierstück eingeschraubt wird. Die Abdichtung erfolgt mit einer PTFE-Dichtung (Ø außen $12 \mathrm{~mm}$ ). Nun kann die Berstscheibe montiert werden. In die obere Öffnung des Zwischenstückes wird die Feinsilberdichtung ( $\varnothing$ außen $40 \mathrm{~mm}$ ) eingelegt. Darauf wird die Berstscheibe mit der Wölbung nach außen (mit der Druckrichtung) gesetzt. Es ist darauf zu achten, dass die Berstscheibe mittig über der Öffnung liegt. Danach wird die Halteschraube eingeschraubt und mit voller Kraft mit einem Schlüssel der Größe 50 angezogen. Dazu wird ein Schlüssel der Größe 55 zum Kontern am Autoklavenkopf benötigt. Beim Festziehen der Verschraubung darf die Berstscheibe nicht verrutschen, um einer Beschädigung oder einem Herausrutschen beim Druckaufbau entgegenzuwirken. Die Berstscheibe zerplatzt bei 100 bar $\pm 10 \%$. Zur Montage des Manometers am Autoklavenkopf wird eine PTFE-Dichtung (Ø außen $12 \mathrm{~mm}$ ) in eine der drei seitlichen Gewindeöffnungen platziert und das Reduzierstück mit einem Schlüssel der Größe 27 festgezogen. Danach wird in das Reduzierstück die PTFE-Dichtung (Ø außen $20 \mathrm{~mm}$ ) eingelegt und das Manometer mit einem Schlüssel der Größe 22 eingeschraubt. In die zweite Gewindeöffnung wird ein Gewindeadapter eingesetzt und ebenfalls mit einer PTFE-Dichtung (Ø außen $12 \mathrm{~mm}$ ) unterlegt. An diesem Gewindeadapter kommt im späteren Verlauf ein Zwei-Wege-Hahn der Firma Swagelok ${ }^{\circledR}$ zum Einsatz, welcher das Ablassen von Gasen ermöglicht. An der letzten seitlichen Gewindeöffnung des Autoklavenkopfes erfolgt die Montage des Temperaturfühlers, welcher die Temperatur direkt im Reaktionsgemisch ermittelt. Vor dem Einbau des Temperaturfühlers ist die seitliche Feststellschraube am Fühler zu lösen. Zur Abdichtung wird eine Feinsilberdichtung (Ø außen $12 \mathrm{~mm}$ ) benötigt. Diese wird in die Gewindeöffnung eingelegt und danach der Fühler eingeschoben. Durch leichtes Biegen des Fühlers nach 
unten kann der gesamte Fühler nachgeschoben werden. Allerdings ist ein zu scharfes Biegen $\mathrm{zu}$ vermeiden, da sonst das eingebaute $\mathrm{Fe} / \mathrm{Cu} / \mathrm{Ni}$ Thermoelement brechen kann. Der Fühler wird nun mit einem Schlüssel der Größe 17 fest angezogen. Zum Schluss wird die Feststellschraube am Fühler wieder festgezogen. Zuletzt wird die PTFE-Dichtung (Ø außen $51 \mathrm{~mm}$ ) in den Autoklavenkopf eingesetzt, der Autoklavenkopf einschließlich Aufbau mit dem Autoklavenbecher zusammengeschraubt und mit einem Schlüssel der Größe 55 fixiert. Nach Abschluss dieser Arbeiten ist somit das Kernstück des apparativen Aufbaus fertig gestellt. Falls der Reaktor nicht dicht ist, muss die entsprechende Undichtigkeit mit ULITH ${ }^{\circledR}$ lokalisiert werden und gegebenenfalls die Verschraubung nachgezogen werden. In der folgenden Tabelle sind die wichtigsten technischen Daten des Laborautoklaven „Modell II" und des Autoklavenkopfes aufgeführt.

- Autoklavenbecher $300 \mathrm{ml}$ :

Tabelle 4.2.1

Tabellarische Auflistung der Technischen Daten des Autoklavenbechers im Arrangement des Hochdruck-Laborautoklaven „Modell II“:

\begin{tabular}{|c|c|}
\hline Leerraum $\left(\mathrm{cm}^{3}\right)$ & 350 \\
\hline Arbeitsvolumen $\left(\mathrm{cm}^{3}\right)$ & 300 \\
\hline Höhe (mm) & 300 \\
\hline Gewicht (g) & 1900 \\
\hline Arbeitstemperatur & 100 \\
\hline Max. Arbeitsdruck (bar) & 300 \\
\hline Prüfdruck bei $+20{ }^{\circ} \mathrm{C}(\mathrm{bar})$ & 50 \\
\hline Außendurchmesser (mm) & 40 \\
\hline Innendurchmesser (mm) & 64 \\
\hline Durchmesser Kopf (mm) & außen und innen flach \\
\hline Bodenform & 1.4571 \\
\hline Werkstoff & \\
\hline
\end{tabular}




\section{Experimenteller Aufbau I}

Somit ist der Reaktionsraum für die Durchführung von Polymerisationen gasförmiger Monomeren komplettiert. Es fehlen nun noch die Verbindungen des Reaktors mit der Gasflasche und das Arrangement, um eine Massenbestimmung der Monomere und eine Temperaturregelung durchführen zu können.

- Autoklavenkopf II:

Tabelle 4.2.2

Tabellarische Auflistung der technischen Daten des Autoklavenkopfes im Arrangement des Hochdruck-Laborautoklaven „Modell II“:

\begin{tabular}{|c|c|}
\hline Gewicht (g) & 1250 \\
\hline Max. Arbeitsdruck (bar) & 200 \\
\hline Arbeitstemperatur & -60 bis $+300^{\circ} \mathrm{C}$ \\
\hline Außendurchmesser (mm) & 64 \\
\hline Werkstoff & 1.4571 \\
\hline
\end{tabular}

\subsubsection{Zubehör und Erweiterungen des experimentellen Aufbaus}

In diesem Kapitel soll die Anordnung von Verbindungsstücken, Ventilen, Kupplungen und Absperrhähnen erläutert werden. Um den im Kapitel 4.1 gestellten Anforderungen an den experimentellen Aufbau gerecht zu werden, wurde folgende Anordnung entwickelt (Abbildung 4.2.1). Es wird für die Verbindungen der einzelnen Komponenten untereinander entweder ein flexibler PFA-Schlauch oder ein Gewebeschlauch eingesetzt. Der PFA-Schlauch besitzt bei einer Temperatur von $20{ }^{\circ} \mathrm{C}$ eine Druckbeständigkeit von maximal 19 bar und ermöglicht eine räumliche Verlagerung des apparativen Aufbaus. Aufgrund der starken Temperaturabhängigkeit der Druckbeständigkeit der PFA-Schläuche (Abbildung 4.2.2) können alle PFA-Schläuche während der Aufheizphase und der Durchführung von Polymerisationen vom Reaktionsraum getrennt werden. Die Reaktionstemperatur wird durch den externen Temperaturregler WRX $2000^{\circledR}$ reguliert. Dieser stellt die Heizleistung an der Heizhaube S $30^{\circledR}$ ein, welche den Autoklavenbecher komplett umschließt und somit eine gleichmäßige Erwärmung garantiert. In der Heizhaube S $30^{\circledR}$ ist ebenfalls das Rührwerk integriert. Die Kraftübertragung erfolgt magnetisch. Der Rührmagnet zeichnet sich durch einen „Turbo-Rühreffekt“ aus und 
erzielt eine gute Durchmischung im gesamten Reaktionsraum. Die Zwei- und Drei-WegeKugelhähne werden von der Firma Swagelok ${ }^{\circledR}$ bezogen.

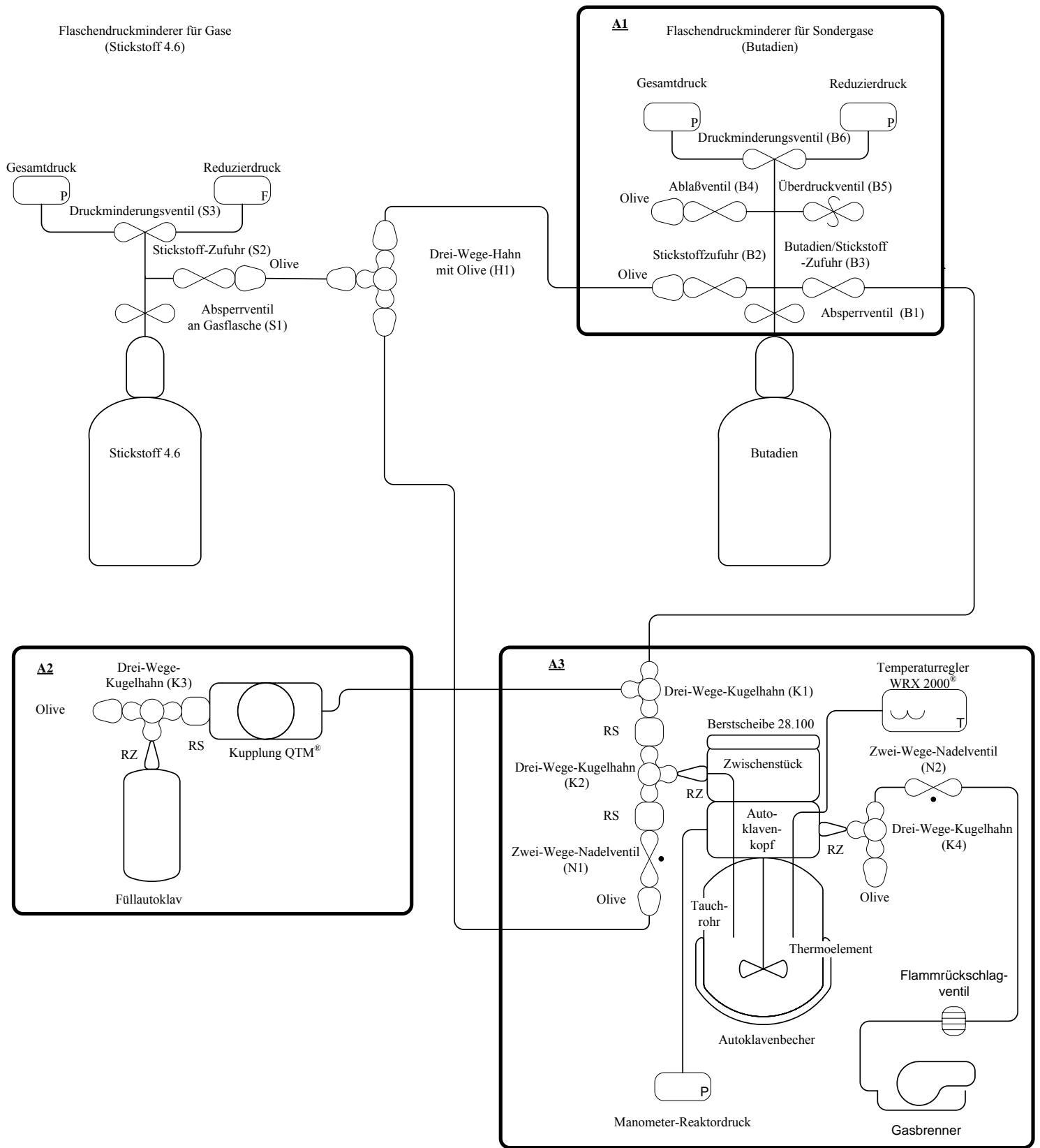

\section{Abbildung 4.2.1}

Schematischer Aufbau des „kleinen“ Autoklaven zur Polymerisation von 1,3-Butadien: Die Größenverhältnisse der einzelnen Komponenten entsprechen nicht den wirklichen Maßstäben. $R Z \rightarrow$ Rohrverschraubung; $R S \rightarrow$ Rohrstück 


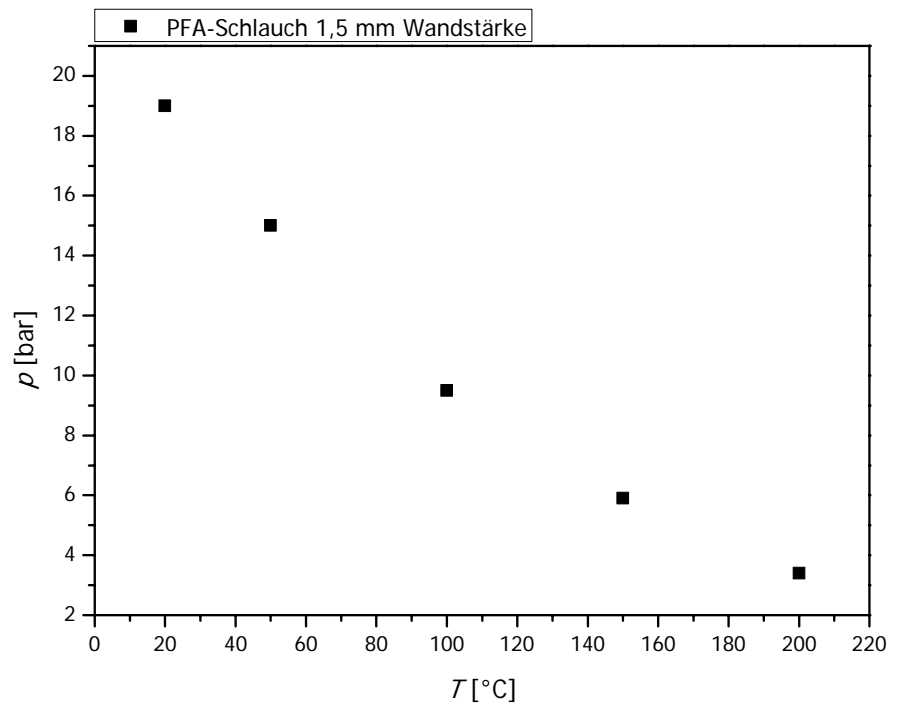

\section{Abbildung 4.2.2}

Darstellung der Druckbeständigkeit der verwendeten PFA-Schläuche mit 1,5 mm Wandstärke in Abhängigkeit der Temperatur.

In Abbildung 4.2.1 ist das gesamte Schema des experimentellen Aufbaus wiedergegeben. In den folgenden Abschnitten werden die Rahmen A1 bis A3 näher beschrieben.

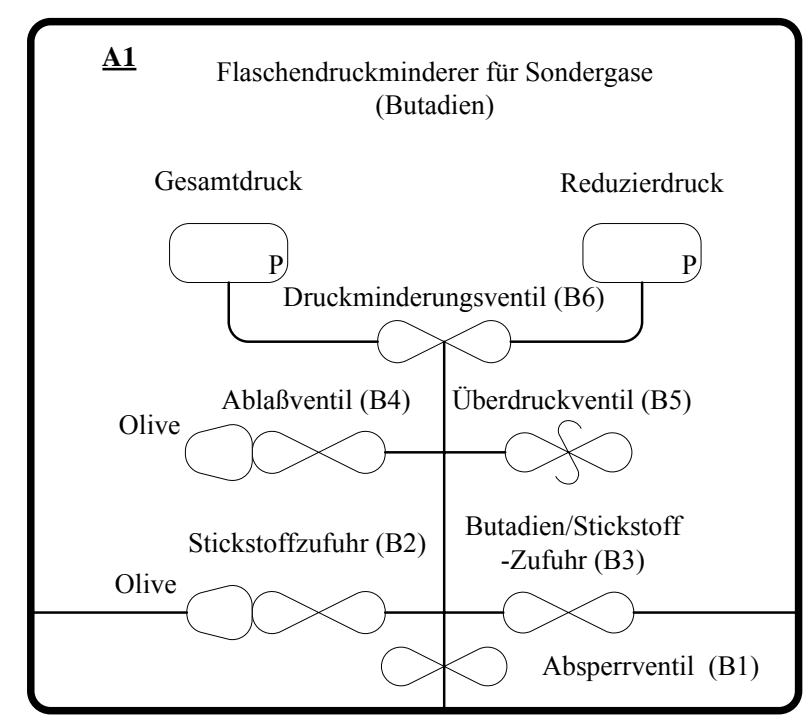

Abbildung 4.2.2

Schematische Zeichnung des Reduzierventils mit intrigierter Spülgasvorrichtung. 
In Abbildung 4.2.2 ist der Flaschendruckminderer (A1) für Sondergase der Firma Messer Griesheim $^{\circledR}$ schematisch abgebildet. Der Flaschendruckminderer (A1) besteht aus Edelstahl und zeichnet sich durch eine hohe Beständigkeit gegenüber korrosiven Gasen aus. Er verfügt über eine Spüleinrichtung und wird direkt an die Gasflasche mit 1,3Butadien angeschlossen. Der Stickstoffstrom wird vom Flaschendruckminderer der Stickstoff-Gasflasche über einen Drei-Wege-Hahn (H1) geleitet. Es besteht also die Möglichkeit den Stickstoffstrom entweder zum Reaktionsautoklaven oder zum Flaschendruckminderer (A1) zu führen. Die Anordnung (A1) kann somit über das Ventil der Stickstoffzufuhr (B2) mit Stickstoff gespült werden.

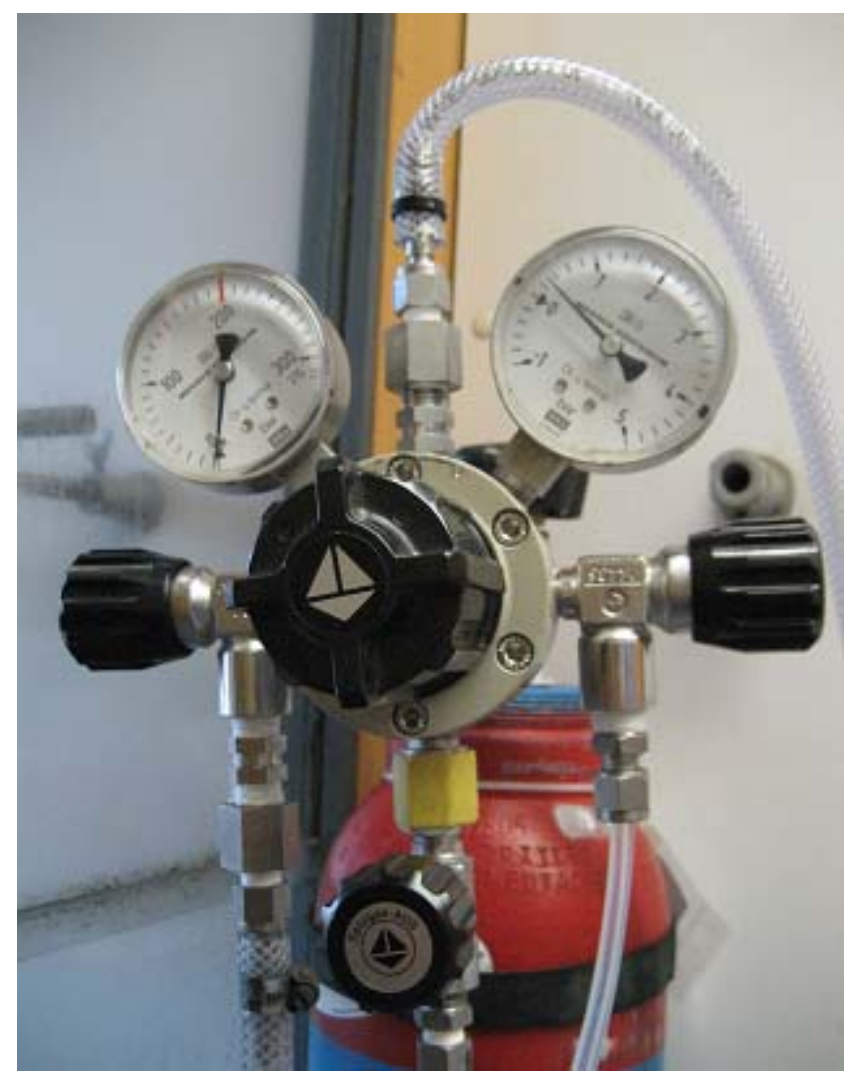

\section{Abbildung 4.2.3}

Fotografie des schematischen Aufbaus A1 (Abbildung 4.2.2).

Weiterhin kann dadurch der PFA-Schlauch und der Füllautoklav (A2) direkt mit Stickstoff geflutet werden. Ein Ablass- (B4), ein Überdruck- (B5) und ein Druckminderungsventil (B6) komplettieren den gesamten Flaschendruckminderer (A1). Das Spülgas kann mit einem Druck von maximal 4 bar eingeleitet werden. Am Überdruck- und Ablassventil, sowie am Ventil zur Stickstoffzufuhr befinden sich 


\section{Experimenteller Aufbau I}

Schlauchadapter, an denen Gewebeschläuche mit einem Innendurchmesser von $8 \mathrm{~mm}$ angeschlossen werden können, die durch Schlauchschellen gesichert werden. Der Anschluss des PFA-Schlauches erfolgt direkt über einen Swagelok ${ }^{\circledR}$-Verschluss.

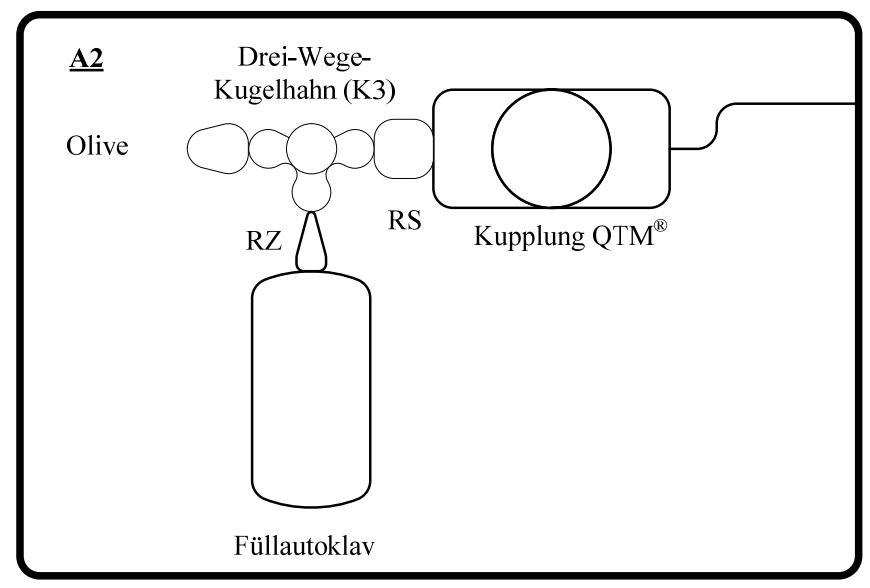

\section{Abbildung 4.2.4}

Schematische Zeichnung des Füllautoklaven mit der Kupplungseinheit QTM ${ }^{\circledR}$ der Firma Swagelok ${ }^{\circledR}$.

In Abbildung 4.2.4 werden der Füllautoklav und das Kupplungssystem näher beschrieben. Durch diese Anordnung ist es möglich, den Füllautoklaven mit Stickstoff zu spülen und danach Butadien einzukondensieren. Der Füllautoklav besteht ebenfalls aus Edelstahl und hat eine Druckbeständigkeit von 500 bar.

Tabelle 4.2.4

Tabellarische Darstellung der technischen Daten des Füllautoklaven:

\begin{tabular}{|c|c|}
\hline Gewicht (g) & 830 \\
\hline Max. Arbeitsdruck (bar) & 500 \\
\hline Aussendurchmesser (mm) & 22 \\
\hline Höhe (mm) & 185 \\
\hline Werkstoff: & 1.4542 \\
\hline
\end{tabular}

In der Deckenkappe des Füllautoklaven wird eine Reduzierverschraubung eingefasst und der Drei-Wege-Kugelhahn (K3) angeschlossen. Die Abdichtung erfolgt mit einem 
Kupferring. Der Drei-Wege-Kugelhahn (K3) besitzt einen Schlauchadapter, an dem ein Gewebeschlauch befestigt ist und ein Rohrstück. An dem Rohrstück wird die Kupplung fixiert, welche dadurch starr am Füllautoklaven sitzt. Der Gewebeschlauch wird direkt in den Abzug geleitet.

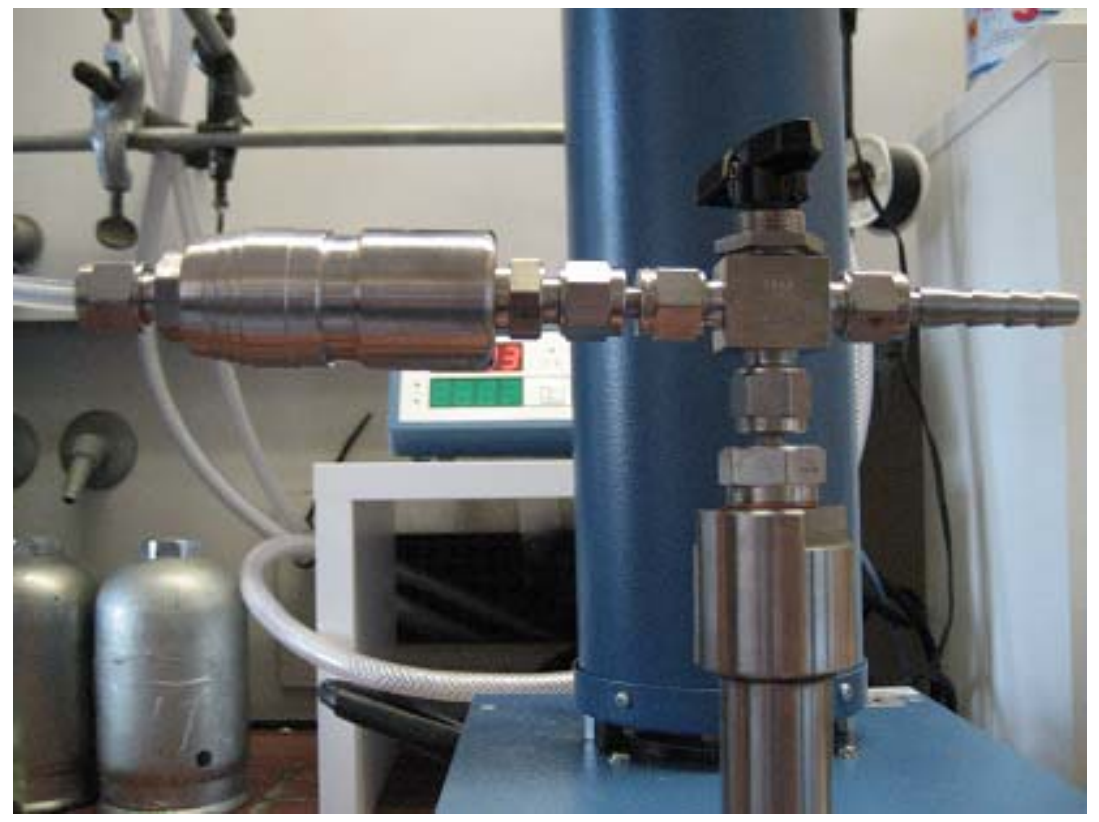

\section{Abbildung 4.2.5}

Fotografie des schematischen Aufbaus A2. 


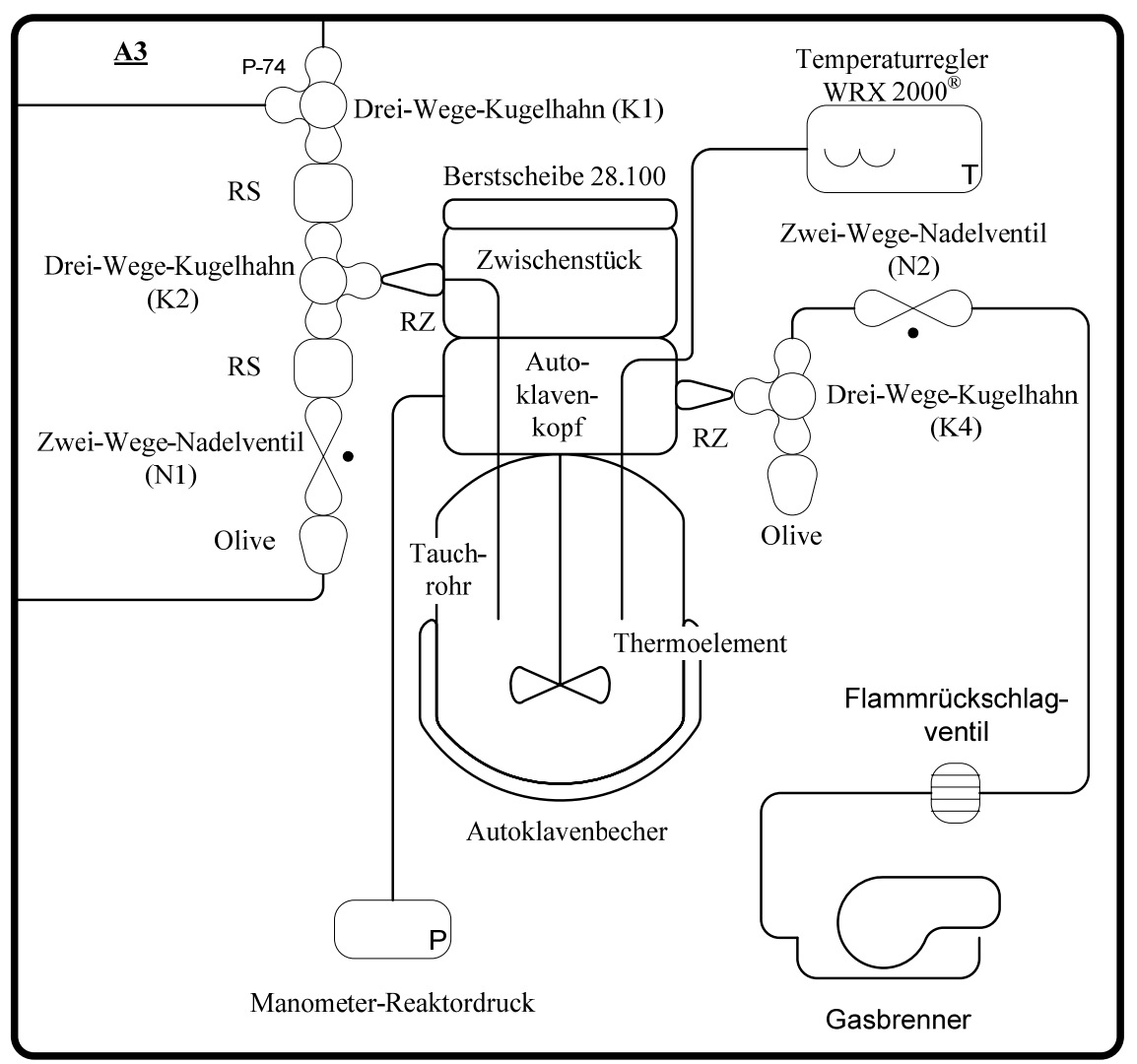

\section{Abbildung 4.2.6}

Schematische Zeichnung des Autoklaven mit Anbauten, Ventilen und Erweiterungen: $R Z \rightarrow$ Rohrverschraubung; $R S \rightarrow$ Rohrstück

In Abbildung 4.2.6 ist neben dem schematischen Aufbau des Reaktionsautoklaven (Kapitel 4.2) auch die Anordnung des entsprechenden Zubehörs am Zwischenstück des Autoklaven „Modell II“ ersichtlich. In die Gewindeöffnung des Zwischenstücks wird eine Reduzierverschraubung eingesetzt, welche mit einer PTFE-Dichtung ( $\varnothing$ außen $12 \mathrm{~mm}$ ) unterlegt wird. An dieser Stelle wird ein Drei-Wege-Kugelhahn (K2) befestigt, der an jeder Seite durch zwei Rohrstücke komplettiert wird. An diese Verbindungsstücke werden einerseits ein weiterer Drei-Wege-Kugelhahn (K1) und andererseits ein Zwei-WegeNadelventil (N1) angeschlossen. An dem Schlauchadapter des Zwei-Wege-Nadelventil (N1) wird ein Gewebeschlauch angebracht (Abbildung 4.2.7), welcher zum Drei-WegeHahn (H1) führt. Durch diese Anordnung besteht die Möglichkeit, den gesamten Reaktionsraum mit Stickstoff zu spülen. Am Drei-Wege-Kugelhahn (K1) werden die PFA-Schläuche vom Kupplungsgegenstück und vom Ventil (B3) mit Swagelok ${ }^{\circledR}$ Verschraubungen befestigt. Durch diese Anordnung wird die Butadien-Gasflasche vom Reaktionsautoklaven getrennt. Das Bindeglied stellt der Füllautoklav dar, wodurch zwei 
Vorteile entstehen. Erstens ist eine exakte Massenbestimmung von Butadien im Milligrammbereich auch mit einer Waage möglich, die einen kleineren Massenbereich besitzt. Die Anschaffung einer teuren Waage, um den gesamten Reaktionsaufbau (ca. 7 kg) mit Milligrammauflösung $\mathrm{zu}$ wiegen, entfällt. Zweitens sind die Mengen an 1,3-Butadien, welche einkondensiert werden, vorher bekannt, wodurch sich die Wiederholung des Experimentes vereinfacht. Die Einstellung der Monomerkonzentration und damit die Anpassung der Initiator- und RAFT-Agens-Konzentrationen werden somit erleichtert.

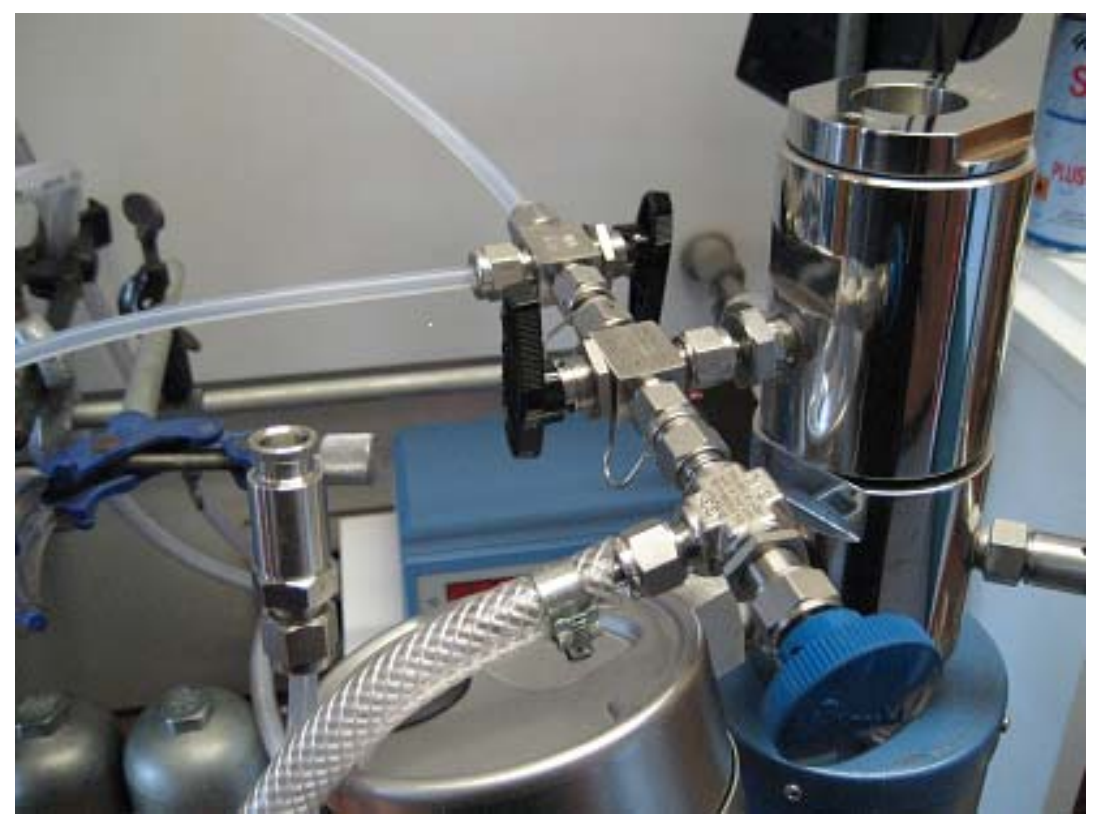

\section{Abbildung 4.2.7}

Fotografie des Autoklavenkopfes mit Anbauten, Ventilen und Erweiterungen.

\subsubsection{Homopolymerisationen von Butadien}

In diesem Abschnitt sollen die Arbeitsschritte für die Durchführung von Homopolymerisationen mit Butadien erläutert werden. Zuerst wird in einem Dewargefäß aus Chromnickelstahl $(\mathrm{V}=2000 \mathrm{ml})$ eine Aceton-Trockeneis-Mischung hergestellt, welche auf eine Temperatur von $-20^{\circ} \mathrm{C}$ eingestellt wird.

Der Füllautoklav wird dann über das Kupplungssystem mit dem Drei-Wege-Kugelhahn (K1) verbunden. Die Spülvorgänge des Füllautoklaven mit Stickstoff werden über die offenen Ventile (S1), (S2), (H1), (B2), (B3), (K1) und (K3) realisiert. Die Drei-WegeKugelhähne müssen dabei immer in Richtung des nächsten Ventils bzw. Kugelhahns 


\section{Experimenteller Aufbau I}

geschaltet sein. Danach wird das Ventil (B2) geschlossen und der Drei-Wege-Hahn (H1) in Richtung (N1) geschaltet. Der Füllautoklav wird nun im Dewar auf - $20{ }^{\circ} \mathrm{C}$ gekühlt und das Ventil (B3) geöffnet. Es erfolgt die Einkondensation von Butadien in den Füllautoklaven. Nach Beendigung der Einkondensation wird der Drei-Wege-Kugelhahn (K3) geschlossen und der Füllautoklav vom System getrennt. Dieser kann nun auf einer Waage (Omnilab ${ }^{\circledR}$ OC 3100-P) abgewogen werden. Der Drei-Wege-Kugelhahn (K1) muss nicht unbedingt geschlossen werden, da das Kupplungsgegenstück auch nach der Trennung gasdicht abschließt.

Das RAFT-Agens und der Initiator werden auf einer Analysenwaage der Firma Sartorius ${ }^{\circledR}$ (Analytic AC 210 S) in ein Schnappdeckelglas eingewogen. Die Volumenbestimmung des Lösungsmittels erfolgt in einem Messzylinder (500 $\pm 1 \mathrm{ml})$. Der Initiator, das RAFTAgens, das Lösungsmittel und der Rührmagnet werden in dem Autoklavenbecher vorgelegt und der Autoklavenkopf einschließlich Aufbau mit dem Autoklavenbecher zusammengeschraubt und mit einem Schlüssel der Größe 50 angezogen. Danach wird der gesamte Reaktionsautoklav in das Aceton-Trockeneis-Bad gestellt und auf $-20^{\circ} \mathrm{C}$ gekühlt. Die tatsächliche Temperatur im Autoklavenbecher kann am Temperaturregler WRX $2000^{\circledR}$ abgelesen werden. Der Drei-Wege-Kugelhahn (K2) wird in Richtung (N1) geschaltet und das Zwei-Wege-Nadelventil (N1) geöffnet. Durch abwechselndes Öffnen und Schließen des Drei-Wege-Kugelhahns (K4) in Richtung des Schlauchadapters erfolgen mehrere Spülvorgänge des Reaktionsautoklaven mit Stickstoff. Beim letzten Spülvorgang wird der Drei-Wege-Hahn (H1) in Richtung (B2) geschaltet und die Stickstoffatmosphäre im Reaktionsautoklaven auf Normaldruck abgesenkt. Danach sind der Drei-Wege-Kugelhahn (K4) und das Zwei-Wege-Nadelventil (N1) zu schließen. Der Drei-Wege-Kugelhahn (K2) wird nun in Richtung (K1) geschaltet und der Füllautoklav wieder an das System gekoppelt. Die Drei-Wege-Kugelhähne (K3) und (K1) werden in Richtung (K2) geschaltet. Es erfolgt die Einkondensation von Butadien in den Reaktionsraum, wobei der Füllautoklav mit einer Heißluftpistole auf Raumtemperatur erwärmt wird. Nach Beendigung der Einkondensation in den Reaktionsautoklaven werden die Drei-Wege-Kugelhähne (K1), (K2) und (K3) geschlossen und der Füllautoklav vom System getrennt. Nun erfolgt eine Gegenwiegung des Füllautoklaven, um den Massenanteil des Monomeren $\mathrm{zu}$ bestimmen. Bei mehrmaliger Einkondensation von Butadien sind die entsprechenden Arbeitsschritte $\mathrm{zu}$ wiederholen. Nachdem alle Komponenten der Polymerisation in den Reaktionsautoklaven eingebracht worden sind, 
wird dieser in die Heizhaube S $30^{\circledR}$ gestellt. Es sind nun die Rührgeschwindigkeit an der Heizhaube $S 30^{\circledR}$ und die entsprechende Temperaturvorwahl am Temperaturregler WRX $2000^{\circledR}$ einzustellen. Jetzt kann die Polymerisation gestartet werden.

Am Ende der Vorbereitungen wird das Flaschendruckminderungsventil (A1) über die Spüleinrichtung mehrmals mit Stickstoff gespült, um Korrosion an den Komponenten des Ventils (A1) vorzubeugen. Hierzu muss das Absperrventil (B1) geschlossen und die Ventile (B2) und (B4) alternierend geöffnet und geschlossen werden. Zur Beendigung der Spülvorgänge wird das Absperrventil (S1) geschlossen und alle Verbindungsschläuche mit dem Ablassventil (B4) auf Normaldruck gebracht, wobei die Ventile (B2) und (B3) noch geöffnet sein müssen. Zum Schluss werden alle Ventile und Hähne geschlossen.

Es besteht die Möglichkeit im Verlauf der Polymerisationen Proben zu ziehen. Als Vorbereitung muss der Gewebeschlauch vom Zwei-Wege-Nadelventil (N1) getrennt werden. Nun wird der Drei-Wege-Kugelhahn (K3) in Richtung (N1) geschaltet. Durch ein behutsames Öffnen von (N1) kann eine Probe direkt in einem Schnappdeckelglas aufgefangen werden. Nach Beendigung der Probenentnahme sind alle Ventile wieder zu schließen.

Um die Polymerisation zu beenden, wird der Reaktionsautoklav aus der Heizhaube S $30^{\circledR}$ entnommen und im Abzug mit dem Aceton-Trockeneis-Bad auf ungefähr $5{ }^{\circ} \mathrm{C}$ abgekühlt. Nach Erreichen der gewünschten Temperatur wird der Gasbrenner in der Verbrennungskammer (Abbildung 4.2.8) angezündet und der Drei-Wege-Kugelhahn (K4) in Richtung Zwei-Wege-Nadelventil (N2) geöffnet. Das Zwei-Wege-Ventil wird behutsam geöffnet, bis die gewünschte Durchflussmenge an Restmonomer erreicht ist. Die Verbrennung des Restmonomers wird durch den Sichtschlitz beobachtet. Nach Beendigung des Verbrennungsvorganges wird das Zwei-Wege-Nadelventil (N2) und der Drei-Wege-Kugelhahn (K4) geschlossen und der Gasbrenner abgeschaltet. Danach wird der Autoklavenbecher vom Autoklavenkopf getrennt und die Polymerlösung in einen Rundkolben gegossen. Es erfolgt die Zugabe der Stabilisator-Lösung. Die Reinigung des Autoklavenbechers erfolgt mit THF. 


\subsubsection{Statistische Copolymerisation von Butadien(BD) mit Isopren (IP) und} n-Butylacrylat (BA)

Die Durchführung der statistischen Copolymerisation erfolgt ebenfalls im Reaktionsautoklaven. Die Arbeitsschritte und -abläufe sind identisch zur Homopolymerisation. Allerdings erfolgt die Vorlage des von Inhibitor befreiten Comonomers gleichzeitig mit dem Lösungsmittel, dem Initiator und dem RAFT-Agens im Autoklavenbecher. 


\section{Experimenteller Aufbau Teil II}

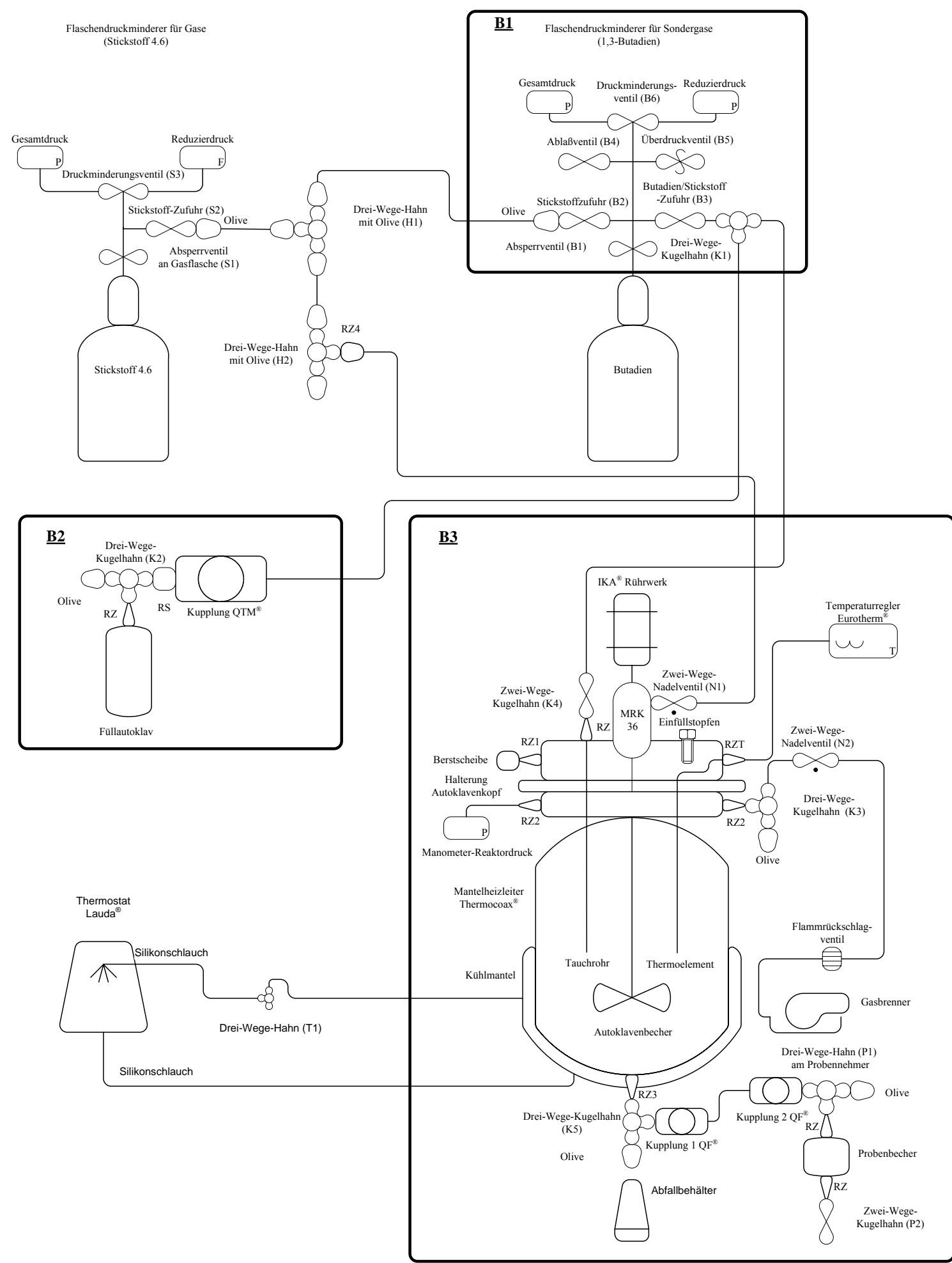


Abbildung 5.0.1

Schematische Gesamtübersicht des experimentellen Aufbaus II: Der Aufbau kann in drei Hauptabschnitte untergliedert werden, welche mit den Kennzeichnungen B1, B2 und B3 versehen sind. Die Abschnitte B1 und B2 sind von der Bedienung und vom Aufbau in großen Teilen zu den Abschnitten A1 und A2 (Abbildung 4.2.1) identisch.

In diesem Kapitel wird die Neukonzeption des Autoklaven II „Modell Conti“ beschrieben. In Abbildung 5.0.1 ist eine Übersicht aufgezeigt, um die Beschreibung und Benennung der einzelnen Komponenten zu erleichtern. Die besonderen Anforderungen an dieses Modell werden in Kapitel 5.1 beschrieben. Aufgrund der Erfahrungen mit dem Autoklaven I „Modell II“ sind einige Neuerungen und Verbesserungen in die Entwicklung dieses experimentellen Aufbaus eingeflossen. Im Kapitel 5.2 wird der technische Aufbau des Autoklavenkörpers beschrieben. Im nächsten Kapitel wird der Zusammenbau des Autoklaven II beschrieben. Die Montage der einzelnen Komponenten des experimentellen Aufbaus kann nicht in willkürlicher Art und Weise erfolgen, da das räumliche Platzangebot dies unterbindet. Im Kapitel 5.4 wird auf die Bedienung des Autoklaven II am Beispiel einer statistischen Copolymerisation von 1,3-Butadien mit Styrol eingegangen.

\subsection{Anforderungen an den Autoklaven II}

Die allgemeinen Anforderungen an eine Apparatur, mit der Flüssiggase oder gasförmige Monomere thermisch polymerisiert werden können, wurden bereits in Kapitel 4.1 beschrieben und haben auch für das neue Modell weiterhin ihre Gültigkeit. Im Rahmen der Industriekooperation entstand der Wunsch nach Polymerproben mit einer Masse von mindestens 80 g. Die Herstellung dieser Proben sollte in einem Polymerisationsdurchgang erfolgen, um Schwankungen in der Probenqualität während der wiederholten Produktion $\mathrm{zu}$ vermeiden. Aus diesem Grund muss das Reaktionsvolumen entsprechend groß dimensioniert werden. Die Erfahrungen mit der Herstellung von Polybutadien und entsprechenden Copolymeren mittels RAFT-Polymerisation ermöglichten eine Abschätzung des benötigten Reaktionsvolumens, welches mindestens $1600 \mathrm{~cm}^{3}$ fassen sollte. Die Hochskalierung des Polymerisationsmaßstabs birgt allerdings gewisse Risiken. Da die zylindrische Form des Autoklaven I übernommen wird, resultiert durch die Erhöhung des Reaktionsvolumens ein verändertes Oberflächen- zu Volumenverhältnis. Der thermische Ausgleich mit der Umgebung, um überschüssige Reaktionswärme abzuführen, kann in diesem Fall nicht so effektiv vollzogen werden. Aus diesem Grund 


\section{Experimenteller Aufbau II}

sollten die Regelintervalle des Thermoreglers individuell auf den entsprechenden Befüllungszustand des Autoklaven angepasst werden können, um ein mögliches „Durchgehen“ der Reaktion zu unterbinden und eine maximale Aufheizrate ohne Überhitzungszonen zum Sollwert zu ermöglichen. Daher ist eine leistungsfähige und gleichzeitig sensible Heizung unerlässlich. Weiterhin ist eine effektive Kühlung des Autoklavenkörpers wichtig, um das Einkondensieren des 1,3-Butadiens bei der Befüllung zu erleichtern. Eine schnelle Abkühlung des Polymerisationsansatzes ist nicht nur für die definierte Beendigung der Polymerisation notwendig, sondern kann auch im Notfall Schlimmeres verhindern. Als weitere Notfalleinrichtung ist eine Berstscheibe erforderlich. Für die gewünschten Reaktionsbedingungen ist ein Berstdruck von 50 bar vorgesehen.

Um die zu untersuchenden Polymerisationssysteme ausreichend genau zu beschreiben, ist eine Probenentnahme während der Polymerisation von Vorteil. Die Probenentnahme ist auch schon beim Autoklaven I möglich, wobei allerdings das geschlossene System geöffnet werden muss. Somit kann bei jeder Probenentnahme 1,3-Butadien entweichen und die Reaktionszusammensetzung nachhaltig verändern. Auch aus gesundheitlichen Aspekten sollte die Expositionsdauer von Menschen mit 1,3-Butadien komplett unterbunden werden. Aus diesen Gründen sollte eine Probenentnahme innerhalb des geschlossenen Systems ermöglicht werden. Generell ist eine Erhöhung der Flexibilität des experimentellen Setups erstrebenswert, um auf veränderte Reaktionsbedingungen oder neue Polymerisationssysteme vorbereitet $\mathrm{zu}$ sein. Beim Autoklaven I mussten das Befüllen, das Spülen mit Stickstoff und die Probenentnahme durch eine einzige Öffnung erfolgen. Aus diesem Grund war ein Umbau oder eine Veränderung am apparativen Aufbau während der Polymerisation nahezu unvermeidbar. Die Erhöhung der Flexibilität resultiert ebenfalls in einer erhöhten Bedienerfreundlichkeit.

Um Polymerisationen mit hochviskosen Medien oder an der Oberfläche von Füllstoffen zu ermöglichen, sollte die Kraftübertragung auf das Rührwerk direkt erfolgen. In diesem Zusammenhang ist auch eine Erhöhung der maximalen Rührleistung wünschenswert. 


\subsection{Konstruktionsmerkmale des Autoklaven II}

In diesem Abschnitt wird die praktische Umsetzung der im Kapitel 5.1 erwähnten Anforderungen beschrieben. In Abbildung 5.0.1 ist eine schematische Gesamtübersicht des experimentellen Aufbaus ersichtlich. Diese Übersicht ermöglicht eine in sich schlüssigere Beschreibung der Apparatur.

In den nachfolgenden Unterkapiteln wird der Druckkörper des Autoklaven II beschreiben. Hierbei wird zwischen den Autoklavenkopf (Kapitel 5.2.1) und dem Autoklavenbecher (Kapitel 5.2.2) unterschieden.

\subsubsection{Autoklavenkopf}

Um dem Wunsch nach einer erhöhten Flexibilität des experimentellen Aufbaus gerecht zu werden, wird der Autoklavenkopf mit einer Vielzahl an Anschlussmöglichkeiten versehen. Die Einkondensation des 1,3-Butadiens erfolgt durch ein Tauchrohr. Weiterhin ist eine Öffnung für das Vorlegen der einzelnen Komponenten des Polymerisationsansatzes vorgesehen, so dass der Autoklavenbecher zum Befüllen nicht vom Autoklavenkopf getrennt werden muss. Auch das Spülen mit Stickstoff sollte über einen separaten Eingang erfolgen. Das Rührwerk wird mittig im Autoklavenkopf integriert, um eine gleichmäßige Durchmischung der Reaktionslösung zu gewährleisten. Weiterhin befinden sich die Berstscheibe, das Manometer, der Zugang für das Thermoelement und der Ausgang für den Druckausgleich und die Entsorgung des Restmonomers im Autoklavenkopf. Diese Zentralisierung ermöglicht zum Einen den einfachen Austausch der Autoklavenbecher und zum Anderen eine feste Installation des Autoklavenkopfes in der Halterung, wodurch Wartungs- und Reparaturarbeiten erleichtert werden. In Abbildung 5.2.1 ist eine schematische Zeichnung des Autoklavenkopfes zu sehen. Insgesamt befinden sich am Autoklavenkopf sieben Anschlüsse. Ein M30x2 Gewinde oben in der Mitte ermöglicht die Aufnahme der Magnetrührkupplung. Die vier seitlichen Öffnungen an den abgeschrägten Seiten dienen zur Aufnahme der Berstscheibe (NPT 1/4“), des Thermofühlers (G 1/4“), des Einfüllstopfens (G 1/4") und eines Einschraubadapters RZ (G 1/4“) für die Befestigung des Zwei-Wege-Kugelhahns (K5). An der äußeren vertikalen Seite befinden sich zwei weitere Öffnungen mit einem G 1/4“" Gewinde. An diesen Stellen können das Manometer und der Drei-Wege-Kugelhahn (K4) installiert werden. Die Befestigung des Autoklavenkopfes an der Halteplatte erfolgt mittels sechs M8 Zylinderkopfschrauben mit Innensechskant. 


\section{Experimenteller Aufbau II}

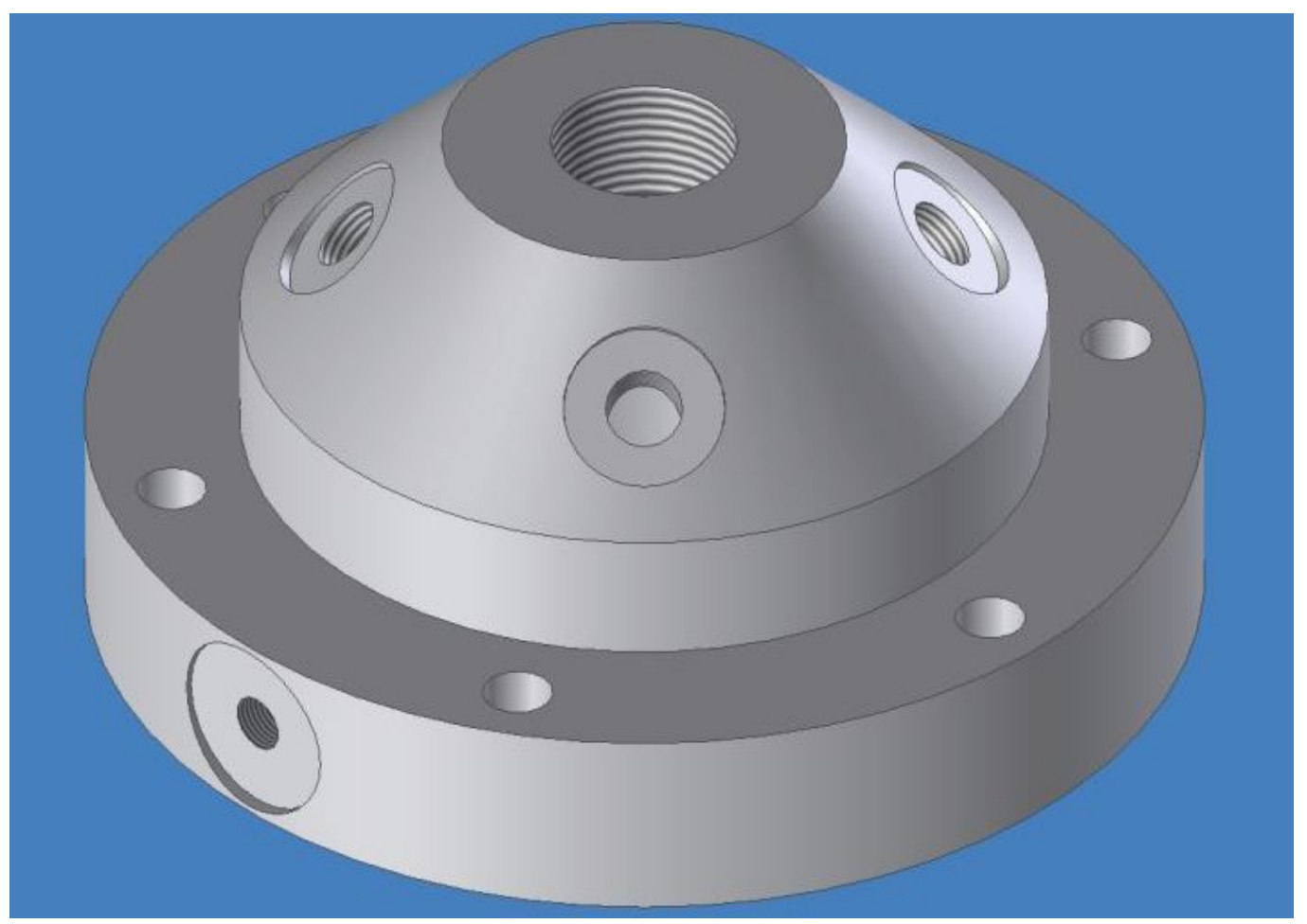

Abbildung 5.2.1

Dreidimensionale Ansicht des Autoklavenkopfes im Entwurf.

Der Autoklavenbecher wird am Autoklavenkopf mit acht M12 Zylinderkopfschrauben mit Innensechskant befestigt, welche in den Befestigungsring geschraubt werden. Dieser Befestigungsring ist ebenfalls aus V4A Edelstahl gefertigt und besitzt einen Außendurchmesser von $166 \mathrm{~mm}$. Der Außendurchmesser ist somit identisch mit dem des Autoklavenkopfes (Abbildung 5.2.2). Der Befestigungsring hat eine Höhe von $20 \mathrm{~mm}$ und besitzt ein Innengewinde von M130x2 für den großen Autoklavenbecher. Bei dem kleinen Autoklavenbecher verringert sich das Innengewinde des Befestigungsringes auf $75 \times 2 \mathrm{~mm}$. Nachdem der jeweilige Befestigungsring mit dem Autoklavenbecher verschraubt ist, kann die Montage mit dem Autoklavenkopf erfolgen. Ein Ring aus PTFE ist in einer Nut am jeweiligen Autoklavenbecher fixiert. Beim Festziehen der acht Verbindungsschrauben wird dieser Dichtring an die plane Unterseite des Autoklavenkopfes gedrückt und somit abgedichtet. Für den großen Autoklavenbecher wird ein Dichtring in den Abmessungen 116x3 mm und für das kleine Arrangement in den Maßen 60x3 mm verwendet. 


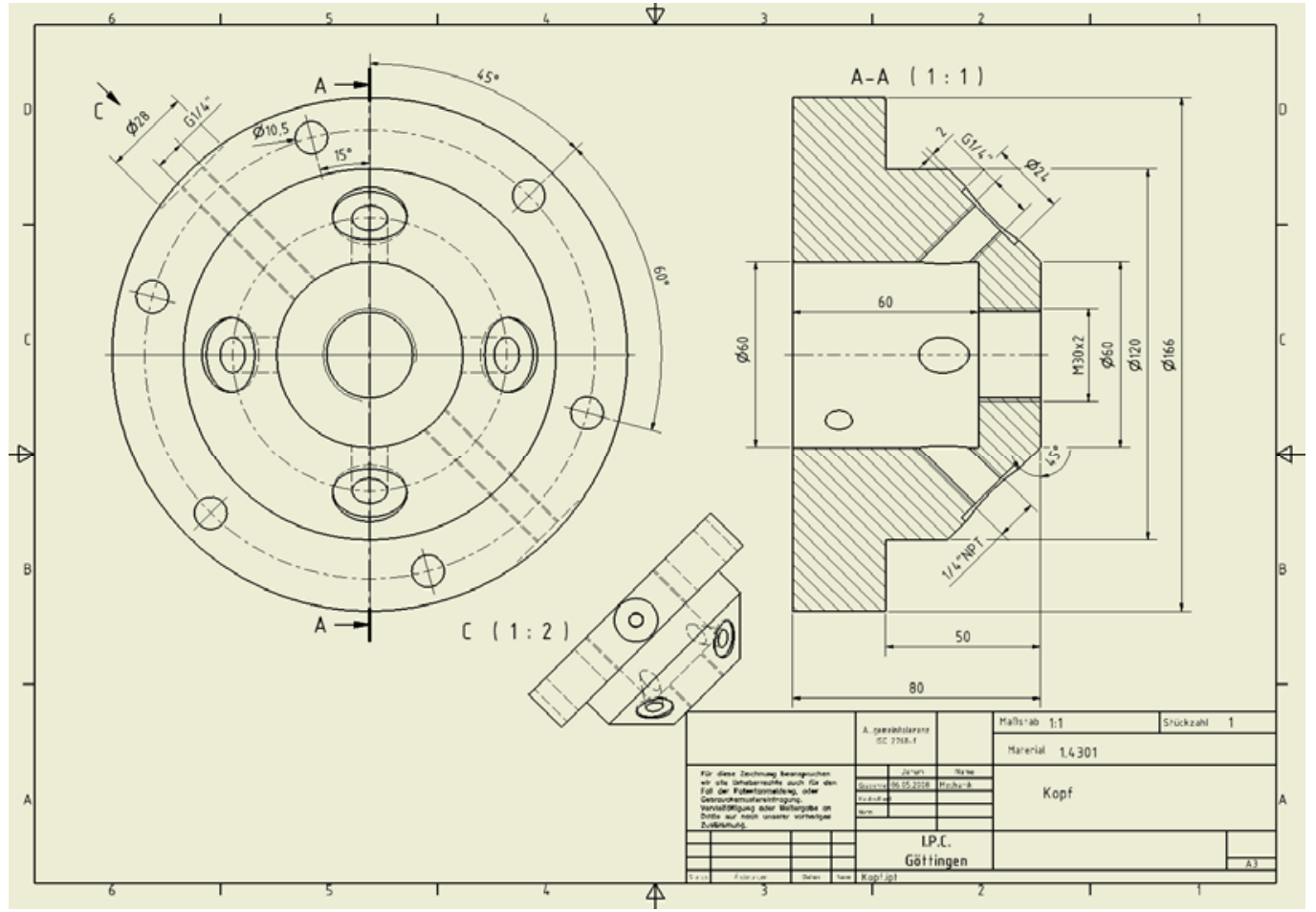

\section{Abbildung 5.2.2}

Technische Zeichnung des Autoklavenkopfes mit der Darstellung der relevanten Abmessungen und Daten: Die Abschrägung an der Oberseite des Autoklavenkopfes erleichtert die Installation und die Bedienung der angebrachten Ventile und Verschlüssen. Die Unterseite des Autoklavenkopfes ist plan gehalten, um eine Abdichtung über einen O-Ring aus PTFE am Autoklavenbecher zu ermöglichen.

\subsubsection{Reaktionsraum und Reaktionsvolumen}

Als maßgebende Weiterentwicklung sollte das Reaktionsvolumen deutlich vergrößert werden. Im Rahmen der experimentellen Untersuchungen ist es allerdings vorteilhaft auch Polymerisationen in kleineren Maßstäben mit 1,3-Butadien durchzuführen, um den Verbrauch an Chemikalien möglichst gering zu halten. Aus diesem Grund sind für die Neuentwicklung zwei Autoklavenbecher vorgesehen. Die Reaktionsvolumina betragen $450 \mathrm{~cm}^{3}$ und $1900 \mathrm{~cm}^{3}$ und können in wenigen Handgriffen getauscht werden. Um dieses Volumenunterschiede zu realisieren, werden die Radien der Zylinder variiert, wobei die Höhe nahezu konstant bleibt. In diesem Fall können also bei einem Tausch der Autoklavenbecher die inneren Einbauten unverändert bleiben und müssen nicht in der Länge angepasst werden. Im Umkehrschluss müssen allerdings die Einbauten, wie das Tauchrohr, der Rührer und das Thermoelement möglichst zentral in der Mitte positioniert werden, damit diese auch im kleineren Autoklavenbecher untergebracht werden können. 
Experimenteller Aufbau II

In den Tabellen 5.2.1 und 5.2.2 sind die Innenabmessungen der unterschiedlichen Autoklavenbecher dargestellt.

Tabelle 5.2.1

Auflistung der Abmaße und der technischen Daten des Druckkörpers des Autoklavenbechers A mit dem kleinen Reaktionsvolumen:

\begin{tabular}{|c|c|}
\hline Innendurchmesser (mm) & 54 \\
\hline Außendurchmesser (mm) & 80 \\
\hline Innenhöhe (mm) & 200 \\
\hline Außenhöhe (mm) & 220 \\
\hline Prüfdruck (bar) & 83 \\
\hline max. Arbeitsdruck (bar) & 50 \\
\hline Arbeitsvolumen (cm $\left.{ }^{3}\right)$ & 450 \\
\hline Werkstoff & 1.4301 \\
\hline
\end{tabular}

Tabelle 5.2.2

Auflistung der Abmaße und der technischen Daten des Druckkörpers des Autoklavenbechers A mit dem großen Reaktionsvolumen:

\begin{tabular}{|c|c|}
\hline Innendurchmesser (mm) & 110 \\
\hline Außendurchmesser (mm) & 140 \\
\hline Innenhöhe (mm) & 207 \\
\hline Außenhöhe (mm) & 227 \\
\hline Prüfdruck (bar) & 83 \\
\hline max. Arbeitsdruck (bar) & 50 \\
\hline Arbeitsvolumen (cm $\left.{ }^{3}\right)$ & 1900 \\
\hline Werkstoff & 1.4301 \\
\hline
\end{tabular}


Der Innenraum des Druckkörpers ist glatt und mit einem flachen Boden versehen. An der Außenseite befindet sich eine spiralförmige Nut mit einer Tiefe von 3,2 mm und einer Breite von 3,0 mm. Diese Nut dient zur Aufnahme des Mantelheizleiters, welcher sich an der Mantelfläche des Druckkörpers befindet. Der Abstand zwischen den einzelnen Nuten beträgt beim großen Autoklavenbecher $6,0 \mathrm{~mm}$ und beim kleineren Pendant 3,0 $\mathrm{mm}$, wodurch eine recht enge Wicklung des Heizleiters ermöglicht wird. Die technischen Spezifikationen der Heizeinrichtung werden im nächsten Kapitel beschrieben.

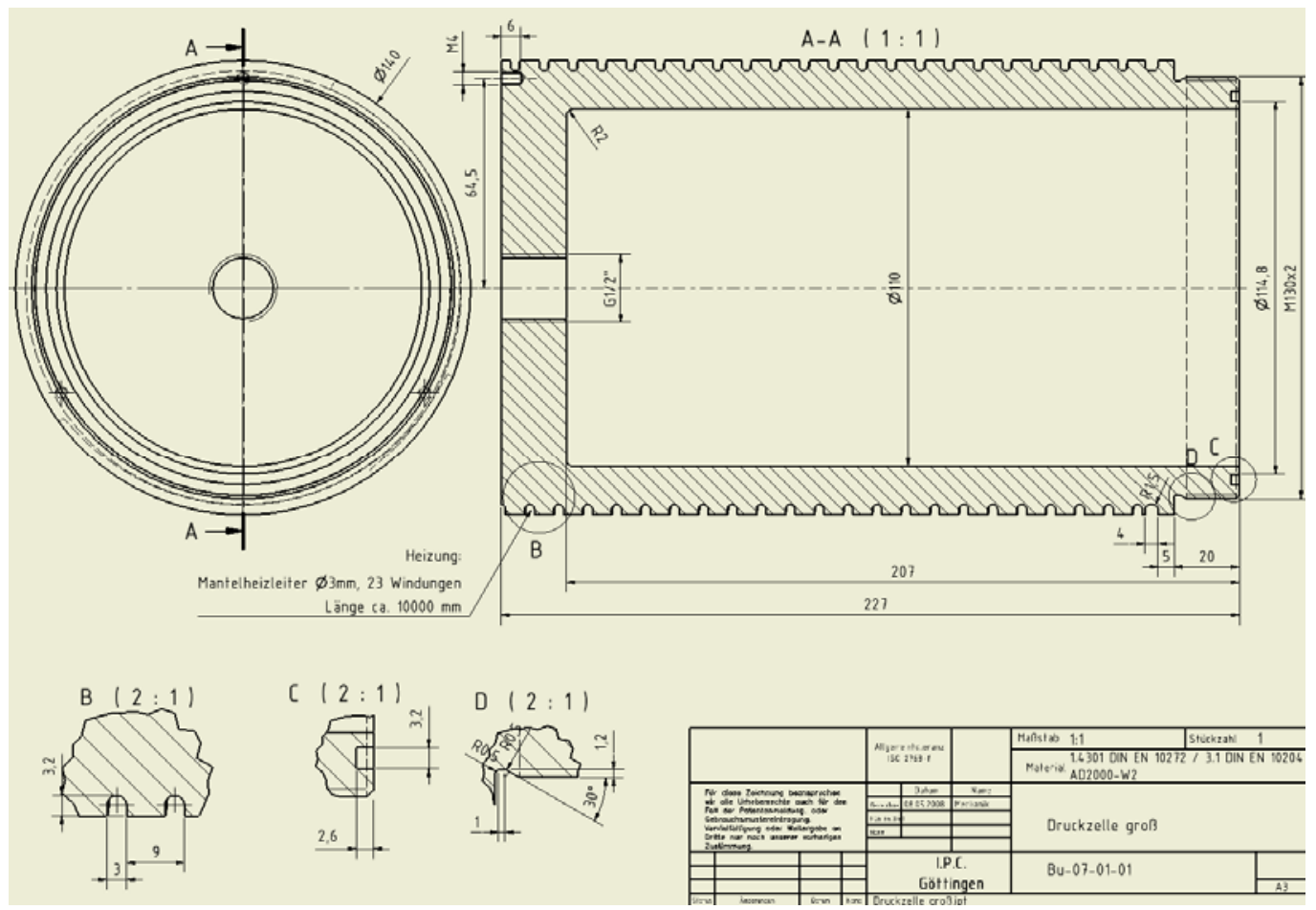

\section{Abbildung 5.2.3}

Technische Zeichnung des Druckkörpers mit einem Innenvolumen von 1900 cm³: An der Unterseite befindet sich das G 1/2" Gewinde für die Aufnahme der Ablassschraube. An der Oberseite ist ein M130x2 Gewinde, auf welches der Befestigungsring geschraubt wird. An der Mantelseite ist die spiralförmige Nut für die Aufnahme des Heizleiters zu erkennen.

Der Innenraum des Druckkörpers ist mit einer Innenzelle versehen, die leicht entfernt werden kann und somit die Reinigung erleichtert. In der Bodenmitte des Einsatzes befindet sich ein G 1/4" Gewinde, um den Auslass zu montieren. Der Boden steigt von dieser Öffnung zu Rand hin in einem Winkel von ca. $17^{\circ}$ an (Abbildung 5.2.6). Somit ist eine restlose Entleerung der Autoklavenbecher möglich, da die Polymerisationslösung immer mittig abläuft. Diese Innenzylinder werden am Boden des Autoklavenbechers 


\section{Experimenteller Aufbau II}

fixiert. Die Druckfestigkeit des Auslasses an der Unterseite des Druckkörpers wird durch einen Einschraubadapter mit einem G 1/2“ Gewinde ermöglicht. Dieser wird von unten in den Boden des Autoklavenbechers mit einem Schlüssel der Größe 34 verschraubt und mit einer Unterlegscheibe aus Kupfer abgedichtet (Abbildung 5.2.4).

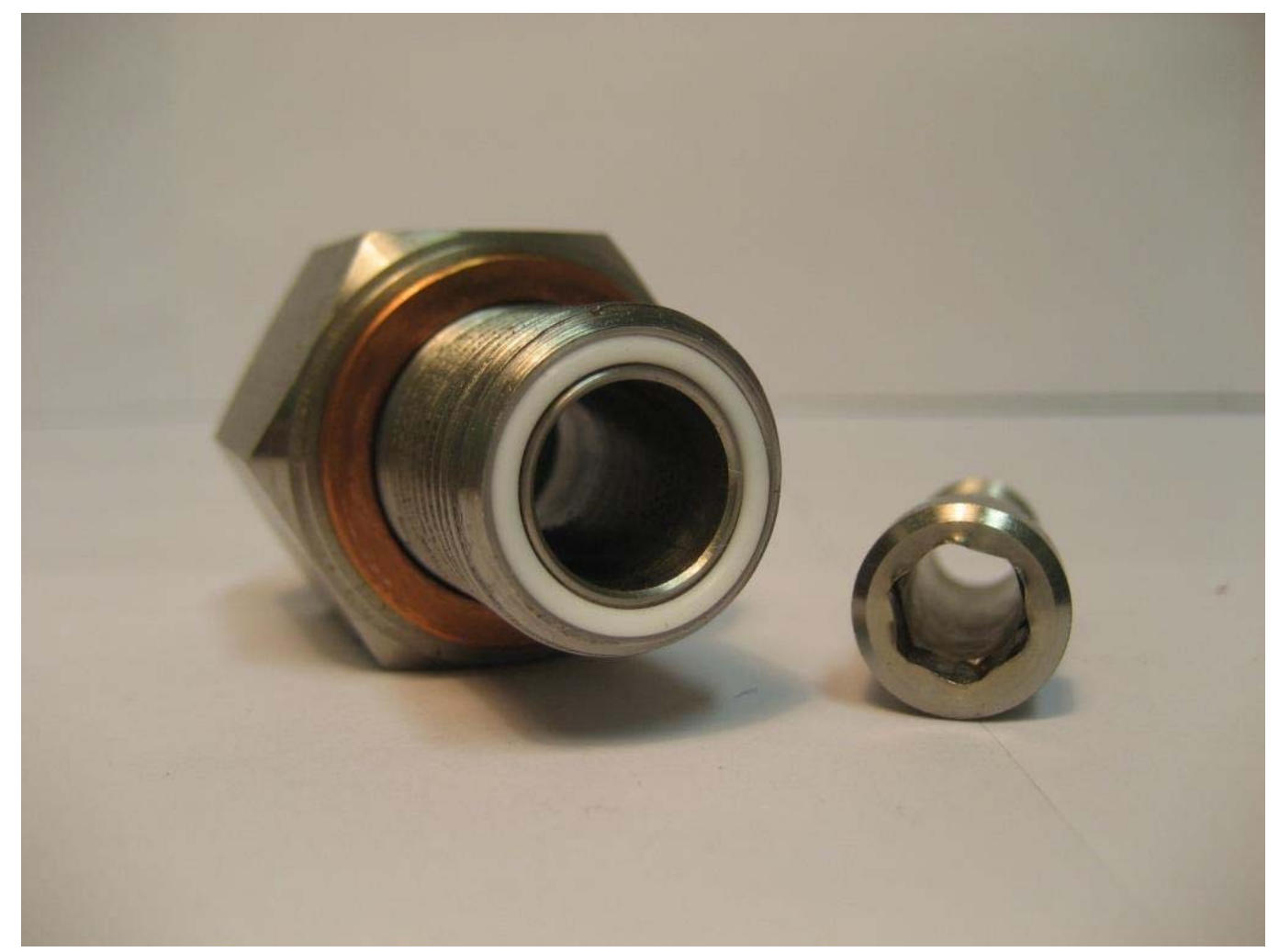

Abbildung 5.2.4

Fotografie des großen Einschraubadapters (RZ3) mit Dichtungsscheibe aus Kupfer und O-Ring aus PTFE: Rechts daneben ist die Sicherungsschraube des Innenzylinders zu sehen.

Dieser Einschraubadapter ist innen hohl und kann auf der Außenseite einen G 3/8“ Einschraubadapter der Firma Swagelok ${ }^{\circledR}$ aufnehmen, welcher dann wieder auf das übliche $6 \mathrm{~mm}$ metrische System führt. Auf der anderen Seite des Einschraubadapters wird ein ORing aus PTFE in einer Nut aufgenommen und schließt bündig mit der Innenkante des Druckkörpers des Autoklavenbechers ab. Wenn der Innenzylinder eingesetzt wird, liegt er auf diesem O-Ring auf. Innerhalb des Einschraubadapters wird eine hohle Schraube mit G 1/4" Gewinde eingesetzt und mit dem Innenzylinder mit Hilfe eines Inbusschlüssels der Größe 6 verschraubt. Durch das Anziehen dieser Schraube wird der Innenzylinder auf den 
O-Ring des großen Einschraubadapters gezogen und dichtet den Innenzylinder zum Druckkörper des Autoklavenbechers hin ab.

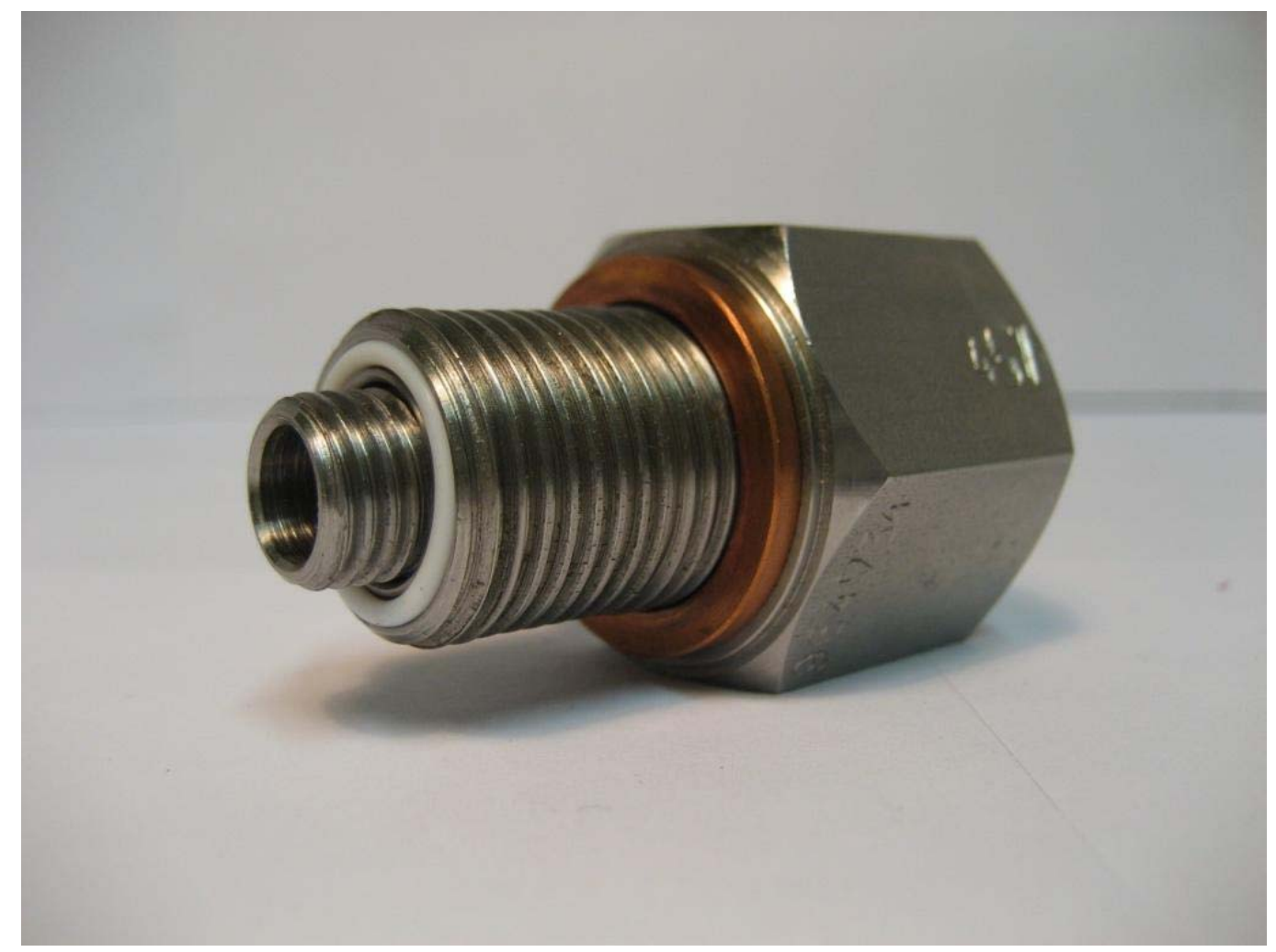

\section{Abbildung 5.2.5}

Fotografie des zusammengesteckten Einschraubadapter (RZ3): Das überstehende G 1/4" Gewinde wird mit dem Boden des Innenzylinders verschraubt.

Beim Ablassen der Polymerisationslösung fließt diese durch den großen Einschraubadapter und die Sicherungsschraube des Innenzylinders. Diese Konstruktion ermöglicht den Einbau von verschiedenen Einsätzen bzw. Innenzylinder mit unterschiedlichen Beschaffenheiten, ohne den Druckkörper tauschen zu müssen. In Abbildung 5.2.6 ist der Innenzylinder des großen Autoklavenbechers zu sehen. Es handelt sich um eine sehr dünnwandige Konstruktion, da der Innenzylinder keine Druckfestigkeit aufweisen muss. Zur Reinigung des Autoklavenbechers kann dieser Einsatz entfernt und separat gereinigt werden. Im Falle eines Verklebens des Innenzylinders mit dem Druckkörper kann der Einschraubadapter RZ3 entfernt werden und kann gegen eine etwas längere Schraube des gleichen Gewindetyps ersetzt werden. Beim Einschrauben dieser Schraube wird die Innenzellen nach oben gedrückt und löst sich vom Druckkörper. 

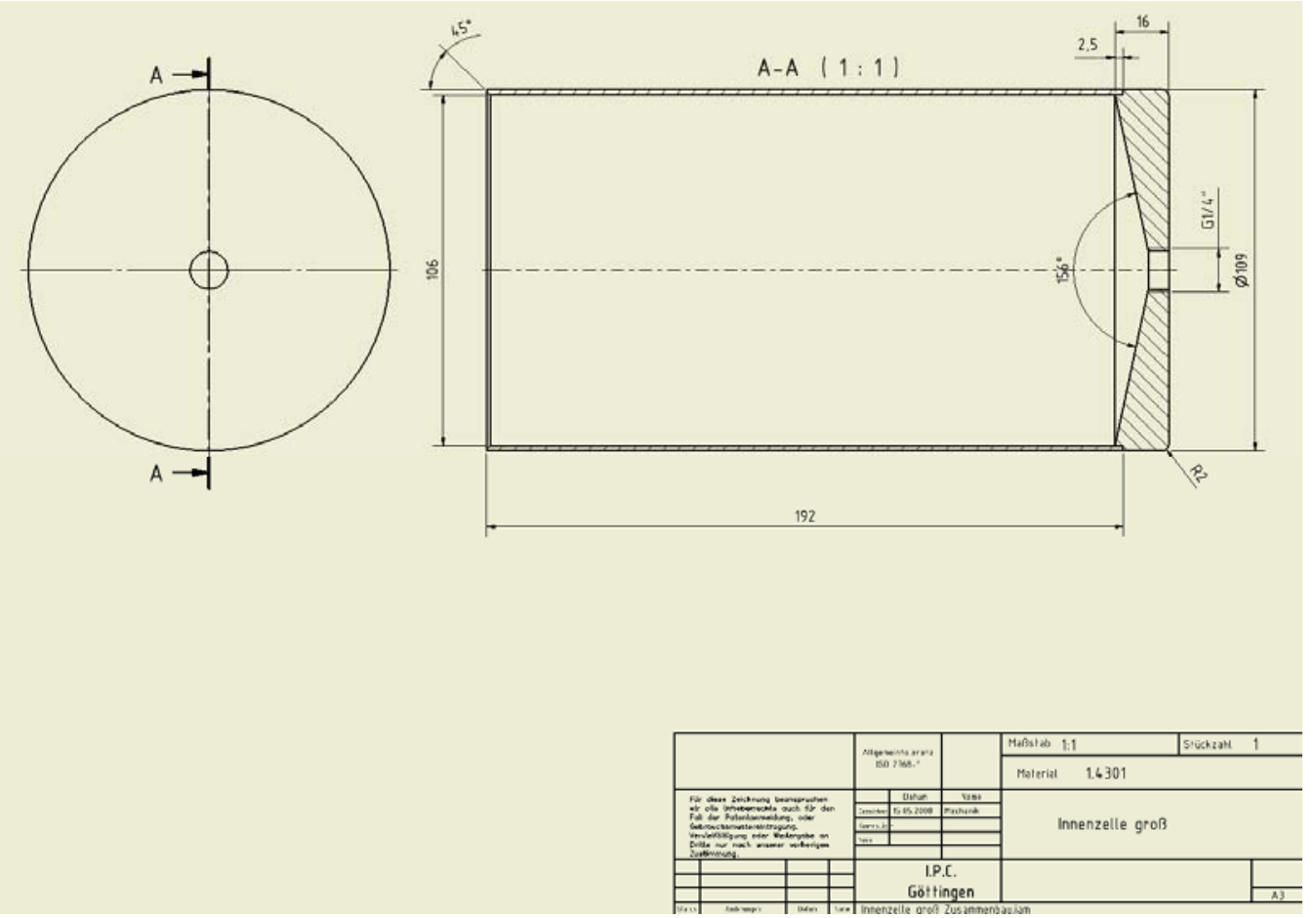

Abbildung 5.2.6

Technische Zeichnung der Innenzelle des großen Autoklavenbechers: Der Boden im Inneren des Einsatzes ist angeschrägt, um ein restloses Abfließen der Reaktionslösung zu ermöglichen.

\subsubsection{Mantelheizleiter und Temperaturregler}

Eine sensible Temperatursteuerung ist bei einer thermisch initiierten Polymerisation unerlässlich. Aus diesem Grund wird die Temperatur direkt in der Polymerisationslösung gemessen. Die Steuerung der Mantelheizleiter erfolgt über einen Thermoregler der Firma Eurotherm $^{\circledR}$ mit einer einstellbaren Regelstrecke. Diese Selbstoptimierung der Regelstrecke ermöglicht es, auch bei unterschiedlichen Befüllungszuständen des Autoklaven eine optimale Aufheizrate zu ermitteln. Für jeden Autoklavenbecher ist ein separater Thermoregler vorhanden. Die Mantelheizleiter befinden sich in unmittelbarer Nähe zum Druckkörper des jeweiligen Autoklavenbechers und werden mit einer engen Wicklung in einer Nut eingelegt, welche sich um die gesamte Mantelfläche des Autoklavenbechers zieht. Als äußere Abdeckung des Mantelheizleiters dient der Kühlmantel, welcher während der Heizphase luftleer ist. Der Mantelheizleiter ohne kalte Enden besitzt einen Durchmesser von $3 \mathrm{~mm}$. In den Tabellen 5.2.3 und 5.2.4 sind die technischen Daten der Mantelheizleiter aufgezeigt. 
Tabelle 5.2.3

Technische Daten des Mantelheizleiters der Firma Thermocoax ${ }^{\circledR}$ ohne kalte Enden für den großen Autoklavenbecher:

\begin{tabular}{|c|c|}
\hline Durchmesser $(\mathrm{mm})$ & 3,0 \\
\hline Länge $(\mathrm{m})$ & 10,0 \\
\hline spez. Widerstand $(\Omega / \mathrm{m})$ & 1,4 \\
\hline Widerstand $(\Omega)$ & 14,0 \\
\hline Leistung $(\mathrm{W})$ & 3778 \\
\hline Spannung $(\mathrm{V})$ & 230 \\
\hline Stromstärke $(\mathrm{A})$ & 16,4 \\
\hline
\end{tabular}

Tabelle 5.2.4

Technische Daten des Mantelheizleiters der Firma Thermocoax ${ }^{\circledR}$ ohne kalte Enden für den kleinen Autoklavenbecher:

\begin{tabular}{|c|c|}
\hline Durchmesser $(\mathrm{mm})$ & 3,0 \\
\hline Länge $(\mathrm{m})$ & 8,0 \\
\hline spez. Widerstand $(\Omega / \mathrm{m})$ & 1,4 \\
\hline Widerstand $(\Omega)$ & 11,2 \\
\hline Leistung $(\mathrm{W})$ & variabel \\
\hline Spannung $(\mathrm{V})$ & 230 \\
\hline Stromstärke $(\mathrm{A})$ & variabel \\
\hline
\end{tabular}

Der Eigenwiderstand des Mantelheizleiters aus Tabelle 5.2.3 ist zu gering, um einen Betrieb mit einer Spannung von $230 \mathrm{~V}$ zu ermöglichen. Aus diesem Grund wird ein Zusatzwiderstand geschaltet, um die Amperezahlen zu verkleinern. Je nach Größe dieses Zusatzwiderstandes kann die Leistung variiert werden. Da der Mantelheizleiter keine kalten Enden besitzt, erfolgt die Isolierung über einen Aufsatz aus Porzellan. 


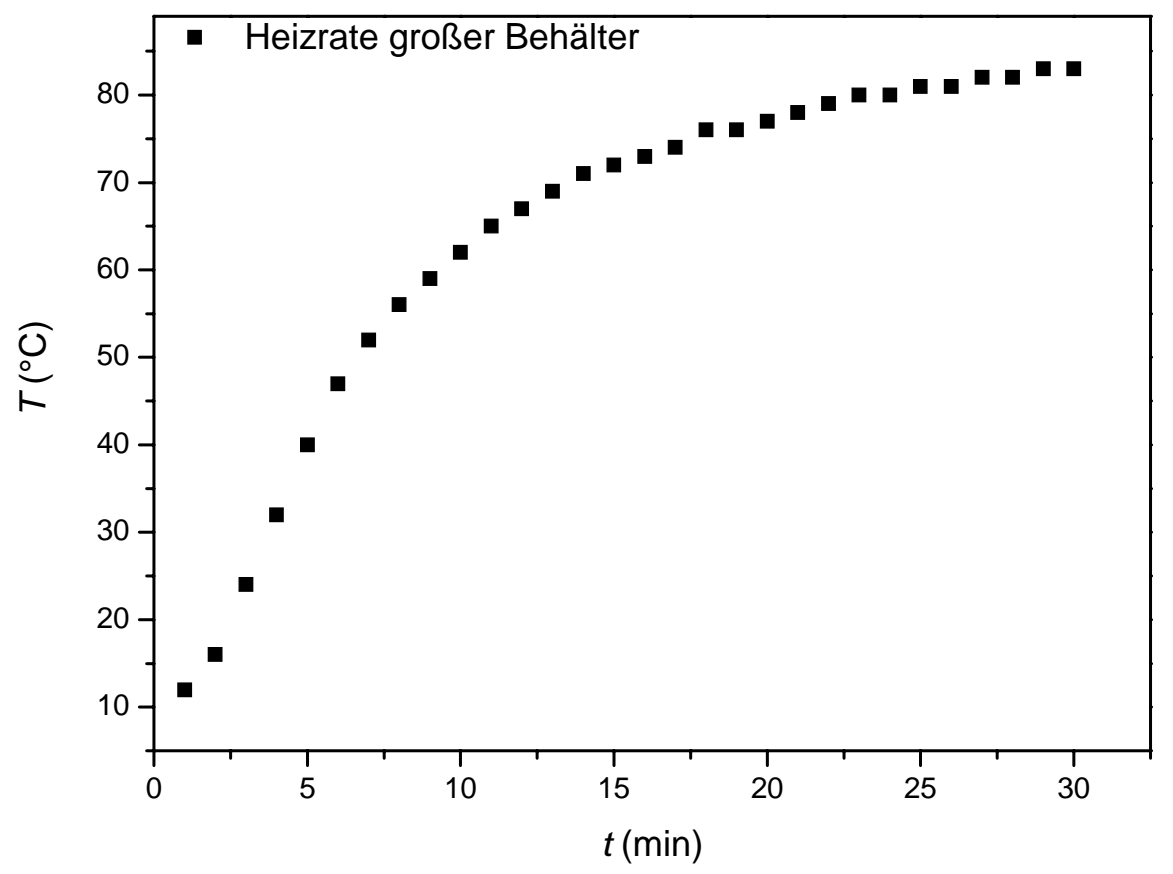

\section{Abbildung 5.2.7}

Auftragung der Ist-Temperatur gegen die Zeit: Nach der Selbstoptimierung der Regelstrecke kann ein Überhitzen des Reaktionsansatzes vermieden werden. Die Aufheizrate ist für den großen Autoklavenbecher mit einem Füllvolumen von ca. $V=950 \mathrm{~cm}^{3}$ und einer Solltemperatur von $T=87^{\circ} \mathrm{C}$ charakteristisch.

Ein Cr-Ni-Cr Thermoelement dient zur Bestimmung der Ist-Temperatur, welches druckfest mit einem Einschrauber der Firma Swagelok ${ }^{\circledR}$ installiert ist, wodurch ein leichter Austausch ermöglicht wird. Das Thermoelement wird am Tauchrohr fixiert. Als Regler dient das Modell 3216 der Firma Eurotherm ${ }^{\circledR}$. Nach der Selbstoptimierung der Regelstrecke kann eine schnelle Aufheizphase ohne ein Überschwingen gewährleistet werden. In Abbildung 5.2.7 ist eine Aufheizphase des großen Autoklavenbechers dargestellt.

\subsubsection{Kühlsystem}

Eine effektive Kühlung erleichtert den Umgang mit dem Autoklaven II. Bei der Einkondensation von 1,3-Butadien ist ein gekühlter Autoklavenbecher ebenso erforderlich, wie zur definierten Beendigung einer thermischen Polymerisation. Die 
Kühlmanschette dient als äußere Abdeckung des Mantelheizleiters. Der Kühlmantel ist aus einer Kupfer-Zink-Legierung gefertigt und ist für einen Kühlmitteldruck von 0,5 bar ausgelegt. Die Anschlüsse an der Kühlmanschette haben einen Durchmesser von $12 \mathrm{~mm}$.

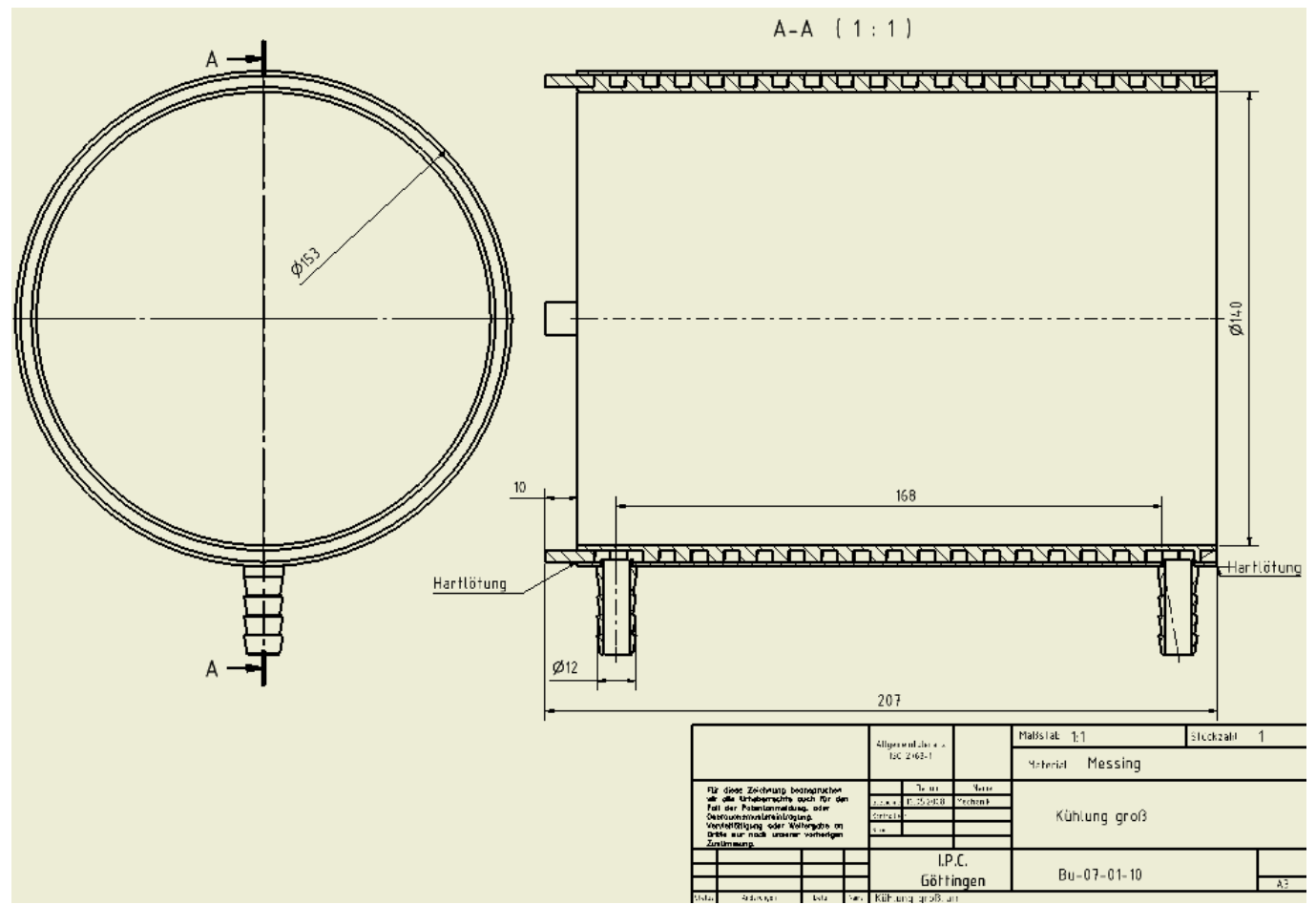

Abbildung 5.2.8

Technische Zeichnung der Kühlmanschette des großen Autoklavenbechers.

Die Verbindung zum Thermostaten erfolgt über Silikonschläuche, welche mittels Schlauchschellen gesichert werden. Im zuführenden Silikonschlauch ist ein Drei-WegeHahn geschaltet. Dieser ermöglicht das Ausleeren des Kühlmantels mit Überdruck, wobei das Kühlmittel in das Reservoir des Thermostaten gedrückt wird. Der Kühlmantel kann jederzeit mit Kühlflüssigkeit in einem Temperaturbereich von $-10^{\circ} \mathrm{C}$ bis Raumtemperatur geflutet werden. Aufgrund der direkten Nähe der Heizung und der Kühlung zur Polymerisationslösung wird eine effektive Wärmeübertragung ermöglicht. In Abbildung 5.2.8 ist der Kühlmantel des großen Autoklavenbechers in einer technischen Zeichnung dargestellt. Die Befestigung des Kühlmantels erfolgt an der Unterseite des Autoklavenbechers. 


\section{Experimenteller Aufbau II}

\subsubsection{Rührwerk für hochviskose Medien}

Die Rührleistung des neuen Autoklaven soll im Vergleich zum ersten Modell um ein Vielfaches erhöht werden. Aus diesem Grund wird der Rührmagnet des kleinen Autoklaven durch einen Rührer mit einer festen Welle ersetzt.

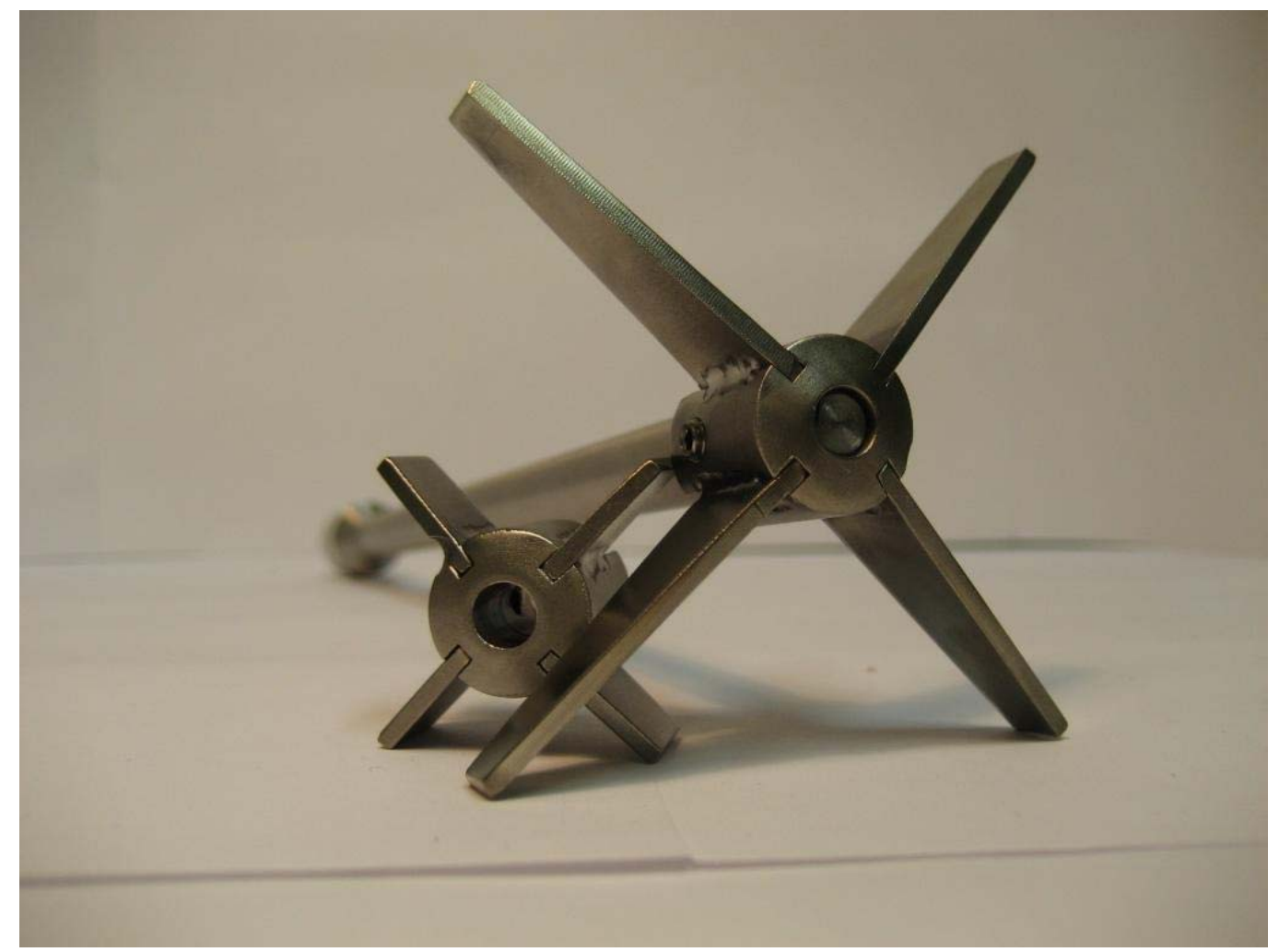

Abbildung 5.2.9

Fotografie der Rührwelle mit aufgesetztem Propeller für den großen Autoklavenbecher.

In Abbildung 5.2.9 ist eine Rührwelle abgebildet. Der Propeller ist mit einer Madenschraube an der Welle befestigt und kann jederzeit gegen andere Propellergrößen und -formen getauscht werden. Die Rührwelle wird an die Wellenaufnahme (Durchmesser $7 \mathrm{~mm}$ ) der Magnetrührkupplung MRK36 mit einer Madenschraube befestigt. Die Spezifikationen und technischen Daten der Magnetrührkupplung sind der Tabelle 5.2.5 zu entnehmen. Diese wird mit dem Autoklavenkopf in das M30x2 Gewinde verschraubt und mit einem Schneidring aus Kupfer abgedichtet. Weiterhin besteht die Möglichkeit der Gaseinspeisung über ein Dosierventil (N1), welches seitlich an der Magnetrührkupplung angebracht ist. 
Tabelle 5.2.5

Auflistung des technischen Daten der verwendeten Magnetrührkupplung MRK36:

\begin{tabular}{|c|c|}
\hline Werkstoff & 1.4435 \\
\hline max. Arbeitsdruck (bar) & 200 \\
\hline max. Temperatur $\left({ }^{\circ} \mathrm{C}\right)$ & 300 \\
\hline max. Drehzahl $(\mathrm{U} / \mathrm{min})$ & 3000 \\
\hline max. Drehmoment $(\mathrm{Ncm})$ & 90 \\
\hline Länge über alles $(\mathrm{mm})$ & 228 \\
\hline
\end{tabular}

Die Magnetrührkupplung ist wiederum außerhalb des Druckkörpers über einen Vierkant mit einer flexiblen Welle verbunden, welche auf der anderen Seite in dem Spannfutter des Rührwerks befestigt wird. Die Kraftübertragung der Magnetrührkupplung ist bis zu einem Drehmoment von 90 Ncm gesichert. Das Rührwerk der Firma Swagelok ${ }^{\circledR}$ verfügt über ein drehzahlunabhängiges Drehmoment von $100 \mathrm{Ncm}$. Die Spezifikationen des Antriebes sind in der Tabelle 5.2.6 aufgelistet. Die Halterung des Rührwerks ist auf der Halteplatte des Autoklavenkopfes befestigt.

Das hohe Drehmoment des Rührwerkes und der Magnetrührkupplung ermöglichen eine gute Durchmischung bei hohen Viskositäten. Der Anstieg der Viskosität mit dem Umsatz und das benötigte Drehmoment für eine typische RAFT-Polymerisation von 1,3Butadien $^{[40-47]}$ wurde abgeschätzt und mit einer Kraftreserve der entsprechenden Komponenten beaufschlagt. 


\section{Experimenteller Aufbau II}

Tabelle 5.2.6

Auflistung der technischen Daten des Rührwerkes IKA ${ }^{\circledR}$ Eurostar power control-vispc P1:

\begin{tabular}{|c|c|}
\hline max. Viskosität (mPas) & 70000 \\
\hline Drehzahlbereich (U/min) & $50-1200$ \\
\hline Drehzahleinstellung & stufenlos \\
\hline Drehmoment (Ncm) & 100 \\
\hline Drehmomentanzeige & ja \\
\hline
\end{tabular}

\subsubsection{Probenentnahme während der Polymerisation}

Als Weiterentwicklung ist auch die Probenentnahme innerhalb eines geschlossenen Systems anzusehen. An der Unterseite des Autoklavenbechers befinden sich ein Einschraubadapter (RZ3) und ein Drei-Wege-Kugelhahn (K5), um die Polymerisationslösung abzulassen. An dieser Stelle kann ein druckfester Probennehmer über eine Schnellkupplung des Typs $\mathrm{QF}^{\circledR}$ angeschlossen werden. Diese Kupplungseinheiten zeichnen sich durch einen großen effektiven Querschnitt aus und ermöglichen somit auch das Ablassen von hochviskosen oder heterogenen Polymerisationssystemen. Aufgrund des Überdrucks im Autoklaven im Vergleich zum Probennehmer wird dieser befüllt. Nach der erfolgreichen Befüllung wird die Kupplung druckfrei geschaltet und der Probennehmer kann dann vom System abgetrennt werden. Die Probennehmer sind bis zu einem Druck von 80 bar und einer maximalen Temperatur von $120{ }^{\circ} \mathrm{C}$ zulässig. Als Material dient ein 1.4301 Edelstahl. Das Innenvolumen der Probennehmer beträgt $25 \mathrm{~cm}^{3}$. 


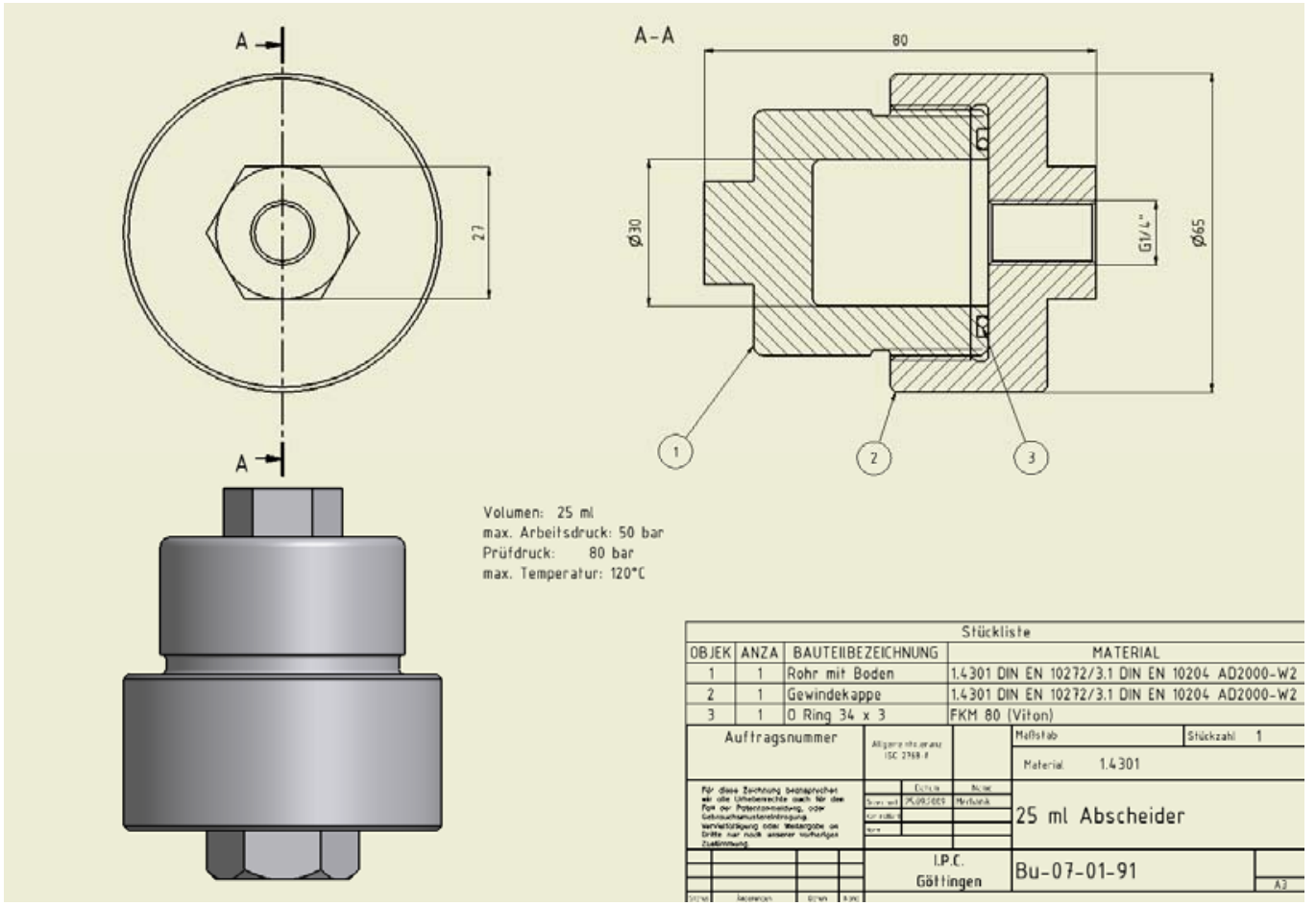

Abbildung 5.2.10

Technische Zeichnung eines Probennehmers zur Entnahme von Polymerproben während der Polymerisation. Der zweiteilige verschraubte Aufbau erleichtert die eventuell erforderliche Reinigung.

In Abbildung 5.2.10 ist ein Probennehmer in einer technischen Zeichnung dargestellt. Der zweiteilige Aufbau des Druckkörpers ist ineinander verschraubt. Die Abdichtung erfolgt hier in analoger Weise zum Autoklaven II. Ein Dichtungsring aus Viton (FKM 80) in der Größe 34x3 mm ist in einer Nut an der Oberseite des Bechers eingefasst. Beim Verschrauben der beiden Teile drückt der Dichtring an die plane Unterseite des Probennehmerkopfes und dichtet somit ab. Ein G 1/4“ Gewinde in der Mitte des Kopfs ermöglicht die Aufnahme eines Einschraubadapters und eines Drei-Wege-Kugelhahnes (P1). An dieser Stelle kann dann die Kupplung des Typs $\mathrm{QF}^{\circledR}$ installiert werden, um einen Anschluss an den Autoklaven zu ermöglichen. Im Gegensatz zur Abbildung 5.2.10 wurden die Probennehmer noch mit einem weiteren G 1/4“ Gewinde bestückt. Dieses befindet sich mittig im Boden des Bechers und ermöglicht wiederum die Aufnahme eines Einschraubadapters bzw. die Aufnahme eines Zwei-Wege-Kugelhahns (P2). Im Laufe der Arbeiten mit dem Autoklaven II hat sich herausgestellt, dass ein Ablassen der Polymerprobe besser $\mathrm{zu}$ handhaben ist als das Aufschrauben des gesamten Probennehmers. 


\section{Experimenteller Aufbau II}

\subsubsection{Sicherheitseinrichtungen}

Als besondere Sicherungseinrichtungen am Autoklaven II selbst sind die Berstscheibe und das Flammrückschlagventil zu nennen. Die Berstscheibe ist auf einen Berstdruck von 50 bar bei $90{ }^{\circ} \mathrm{C}$ konzipiert worden. Dieser Wert entspricht somit dem maximalen Arbeitsdruck des experimentellen Aufbaus. Das Gehäuse und die Berstscheibe wurden bei der Firma Bormann\&Neupert ${ }^{\circledR}$ hergestellt und zeichnen sich durch einen geringen Platzbedarf aus. Die Verschraubung mit dem Autoklavenkopf erfolgt über ein NPT 1/4“ Gewinde. Die Abmessungen das Gehäuse der Berstscheibe betragen $27 \mathrm{~mm}$ in der Höhe und $32 \mathrm{~mm}$ in der Kantenlänge. Der Deckel der Berstscheibe besitzt ein Quermaß von 29 mm und eine Höhe von 13 mm. Das Gehäuse und der Deckel haben die Grundform eines Schraubenkopfes und können mit einem entsprechenden Maulschlüssel eingeschraubt werden. Oberhalb des Gehäuses wird die Berstscheibe druckrichtig eingelegt und mit dem ebenfalls achteckigen Deckel fixiert.

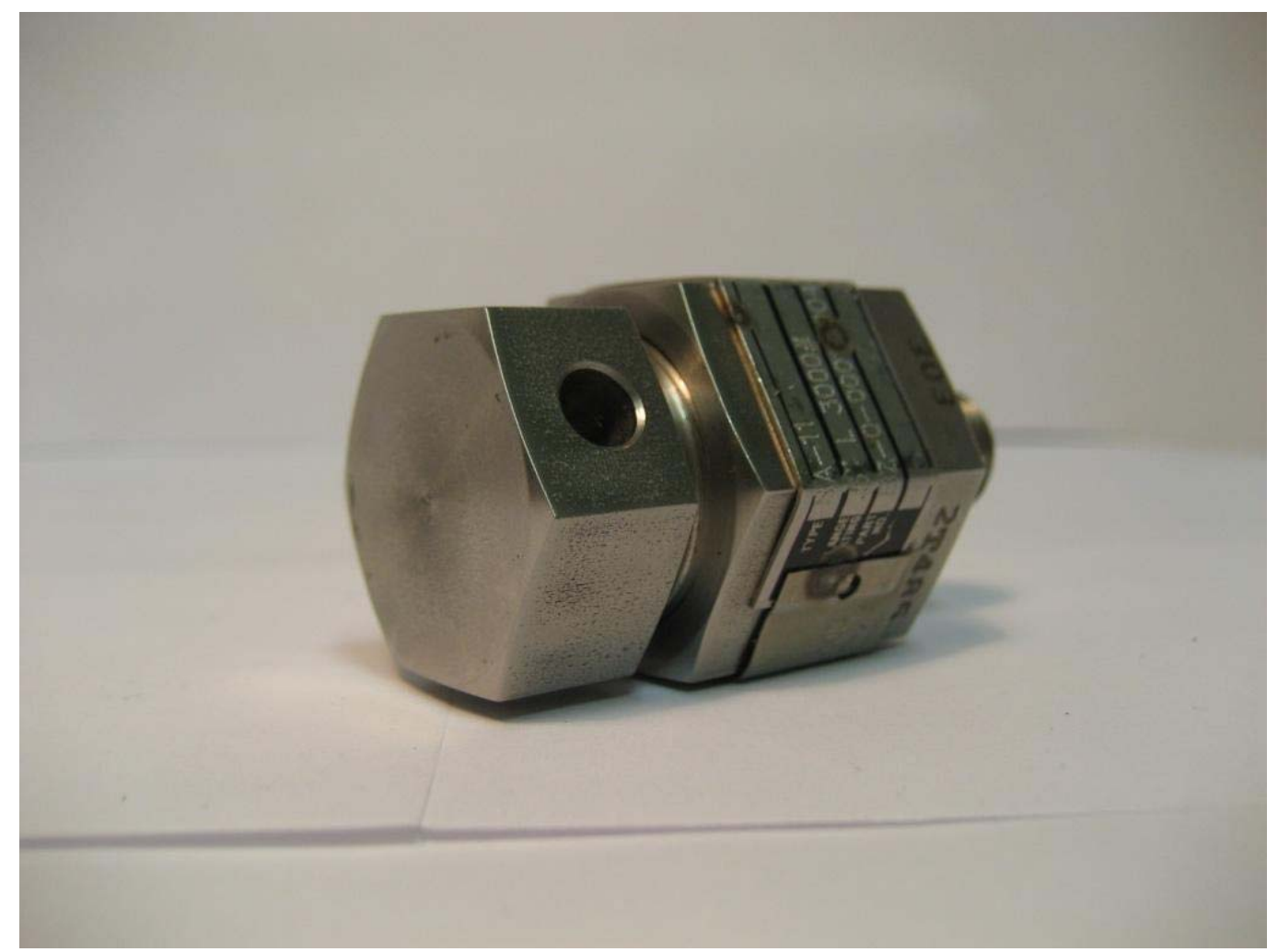

\section{Abbildung 5.2.11}

Fotografie der Berstscheibeneinrichtung: Im Gehäusedeckel sind die seitlichen Ablassöffnungen zu erkennen. Zwischen dem Gehäuse und dem Deckel ist die Berstscheibe installiert. 
Beim Einsetzten und beim Zusammenbau darf die Berstscheibe auf keinen Fall beschädigt werden, da dann die Sicherheitsansprüche nicht erfüllt werden können. Abbildung 5.2.11 zeigt eine Fotografie der Berstscheibe. Im Falle eines Zerplatzens der Berstscheibe entweicht der Überdruck durch die seitlichen Öffnungen des Gehäusedeckels.

Als weitere Besonderheit des experimentellen Aufbaus ist das Vorgehen zur Entsorgung von unverbrauchtem 1,3-Butadien $\mathrm{zu}$ nennen. Aufgrund der toxischen und krebserregenden Wirkung des Monomers ist eine Exposition $\mathrm{zu}$ vermeiden. Ein Verdampfen im Abzug ist möglich, kann allerdings nur für sehr geringe Mengen an 1,3-Butadien durchgeführt werden, da die maximale Belastung der Abluft im Massenstrom nicht höher als 2,5 g/h ${ }^{[48]}$ sein darf. Aus diesem Grund ist die Verbrennung des Restmonomers eine effektive und sichere Variante, um größere Mengen an 1,3Butadien zu entsorgen. Um eine sichere Verbrennung ohne Flammrückschläge in den Autoklaven zu garantieren, wird zwischen dem Zwei-Wege-Nadelventil (N2) und dem Gasbrenner eine Sicherheitseinrichtung mit der Modellbezeichnung RF53N-ES der Firma Witt $^{\circledR[49]}$ geschaltet. Dieses Flammrückschlagventil verhindert den gefährlichen Gasrücktritt und Flammrückschläge nach EN 730/ISO 5175-1 ${ }^{[49]}$. Zwei Einschraubadapter mit einem 1/4“ NPT Gewinde komplettieren die Sicherheitseinrichtung und ermöglichen die Integration in das metrischen System des experimentellen Aufbaus. Eine Verbrennung des Restmonomers sollte allerdings nicht frei im Abzug realisiert werden. Einerseits kommt es $\mathrm{zu}$ einer $\mathrm{zu}$ starken Verschmutzung durch Verbrennungsrückstände und andererseits ist die Verbrennung nicht vollständig. Aus diesem Grund wurde eine gekapselte Kammer konstruiert, um den Bereich der Verbrennung zu minimieren und eine Schornsteineffekt $\mathrm{zu}$ erzielen. Durch die erhöhte Sogwirkung, welche durch diese Kammer erzeugt wird, kommt es zu einer sauberen Verbrennung. 


\section{Experimenteller Aufbau II}

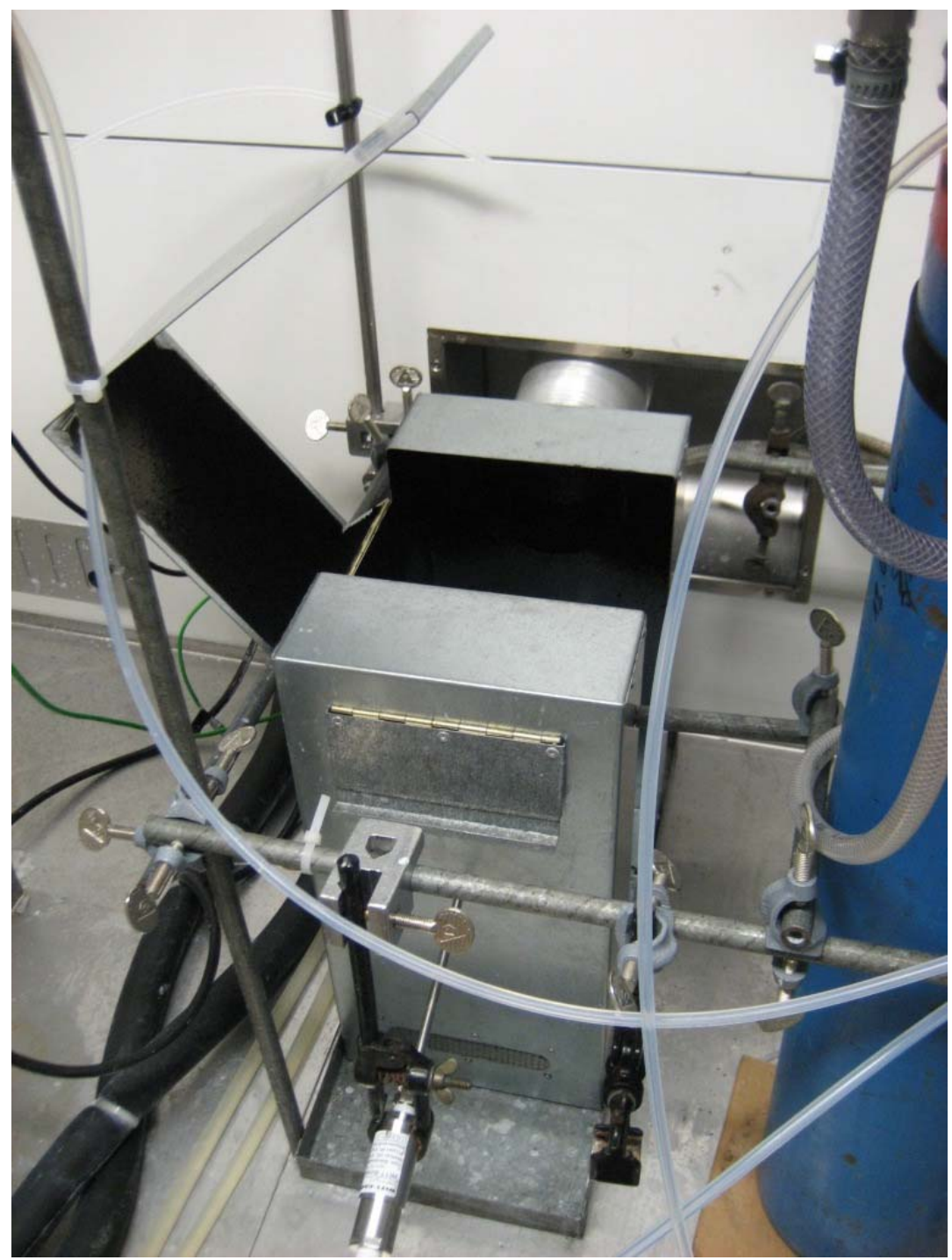

\section{Abbildung 5.2.12}

Fotografie der Verbrennungskammer mit geöffneter Seitenklappe: Im vorderen Bereich ist die Beobachtungsluke und die untere Belüftungsöffnungen zu sehen. Das Flammrückschlagventil der Firma Witt ${ }^{\circledR}$ ist außerhalb der Kammer installiert. Der Gasstrom wird durch ein $6 \mathrm{~mm}$ Edlestahlrohr ins Innere der Kammer geleitet.

In Abbildung 5.2.12 ist eine Fotografie der Umbauung abgebildet. Sie besitzt ein quaderförmiges Grundgerüst mit den Abmessungen $400 \mathrm{~mm}$ x $196 \mathrm{~mm}$ x $300 \mathrm{~mm}$ $(\mathrm{HxBxT})$. An der Vorderseite und den beiden Seitenteilen sind im unteren Bereich Luftschlitze angebracht, welche mit einem feinmaschigen Messinggitter überdeckt sind. Im oberen Bereich der Vorderseite ist eine verschließbare Beobachtungsöffnung installiert, um die Flamme während der Verbrennung zu kontrollieren. Weiterhin gibt es eine große seitliche Klappe in L-Form, um den Gasbrenner in der Umbauung zu 
platzieren und die Entzündung der Flamme zu ermöglichen. An der Rückseite der Kammer befindet sich im oberen Bereich einen Öffnung, welche direkt an die hausinterne Abluft gekoppelt ist. Die Umgebungsluft wird von unten nach oben gesogen und sorgt für eine effektivere und saubere Verbrennung.

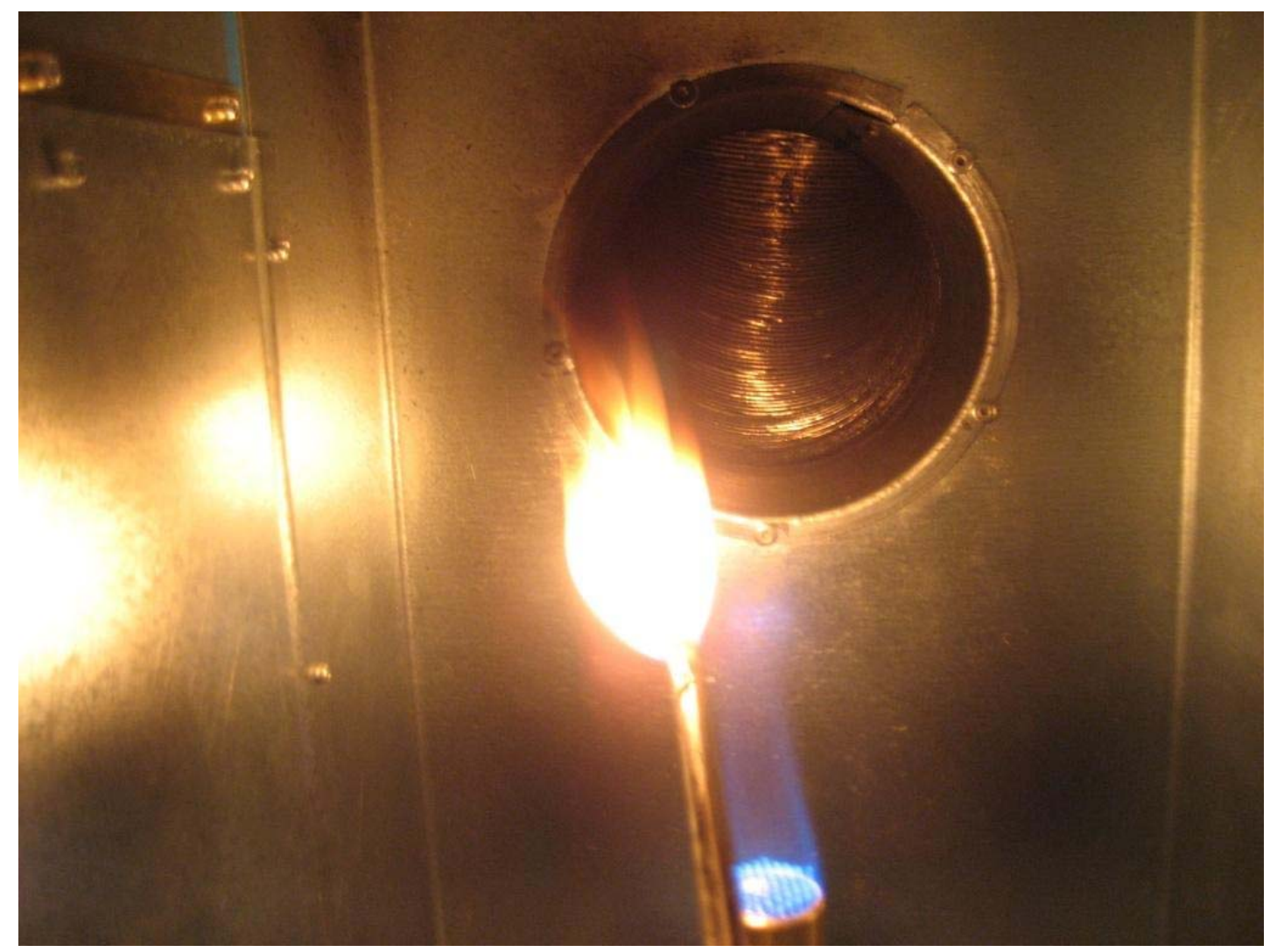

Abbildung 5.2.13

Blick ins Innere der Verbrennungskammer bei der Entsorgung von 1,3-Butadien: Die bläuliche Flamme des Gasbrenners initiiert die Verbrennung des Gasstroms an Restmonomer. 


\subsection{Aufbau des Autoklaven II}

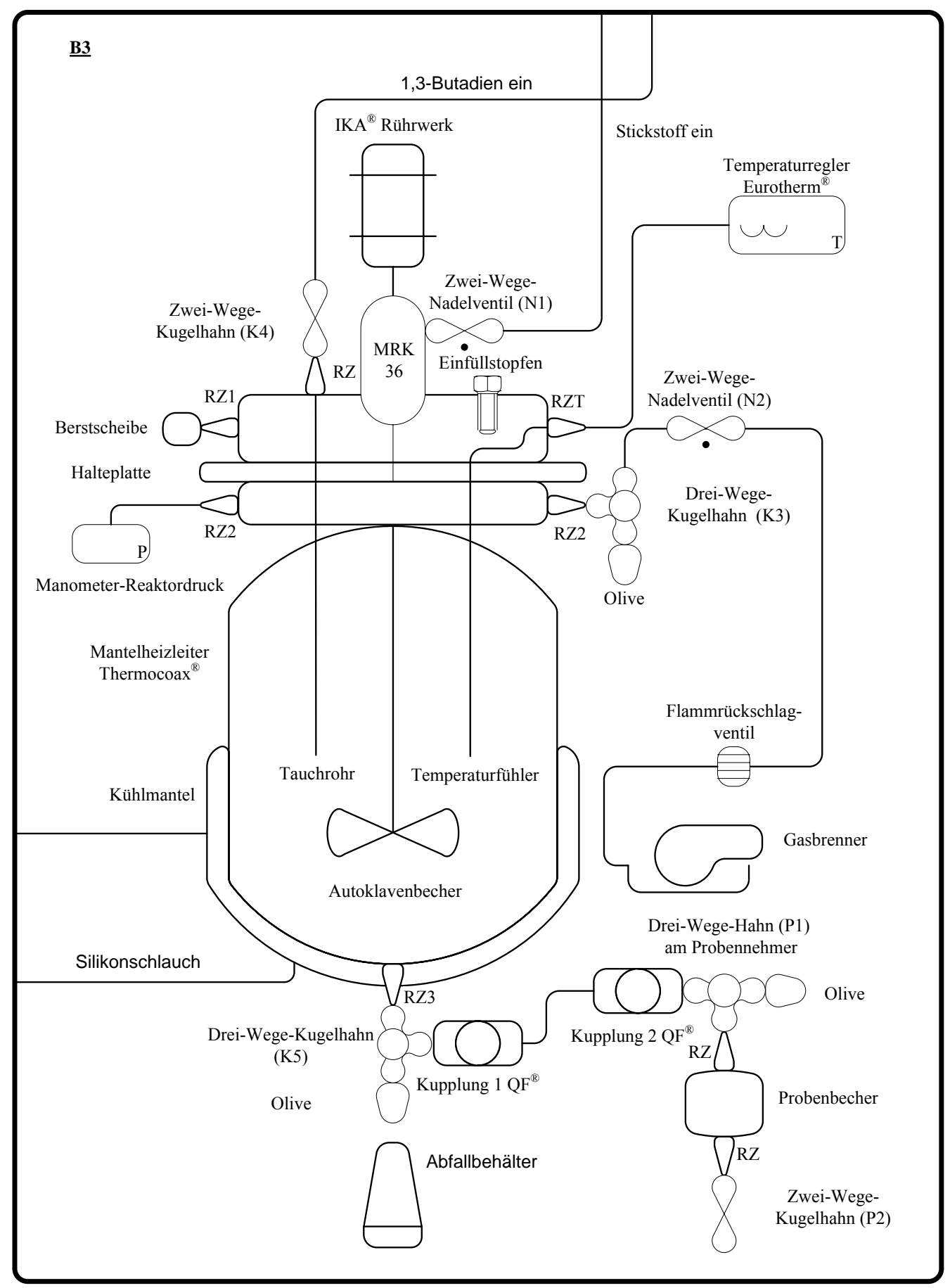

Abbildung 5.3.1

Schematische Übersicht des Abschnittes B3 des Autoklaven II: Die Installation der einzelnen Komponenten erfolgt in einer strikten Reihenfolge. Die Abschnitte B1 und B2 der Abbildung 4.2.1 bedürfen keiner weiteren Erklärung. 
Der schematische Aufbau des Autoklaven II ist in Abbildung 5.3.1 dargestellt. In diesem Kapitel wird nun der Zusammenbau der einzelnen Komponenten erläutert. In der Ausgangsituation ist der Autoklavenkopf an der Halteplatte mit sechs Zylinderkopfschrauben mit Innensechskant befestigt. Weiterhin wird angenommen, dass der Autoklavenbecher, der Mantelheizleiter mit Verkabelung und der Kühlmantel jeweils eine feste Einheit bilden. Die meisten Ventile, Hähne oder Einschraubadapter bedienen sich dem System der Zwei-Klemmring-Rohrverschraubung ${ }^{[50]}$ der Firma Swagelok ${ }^{\circledR}$. Bei diesem System wird zwischen Erstmontage und wiederholter Montage unterschieden. Dies ist bei der Wartung und Montage des Autoklaven II zu beachten. Weiterhin ist es allgemeingültig, dass Gewinde immer mit Teflonband oder in seltenen Fällen auch mit Teflonspray gegen das Kaltverschweißen gesichert werden.

\subsubsection{Montage des Rührwerks und der Magnetrührkupplung}

Im ersten Arbeitsschritt wird die Rührwelle an die Welle der Magnetrührkupplung (MRK36) aufgesetzt und mit einer Madenschraube gesichert. Hierbei ist drauf zu achten, dass die Madenschraube direkt an die abgeflachte Seite der Welle ansetzt. Dann wird das M30x2 Gewinde mit Teflonband umwickelt, um ein Kaltverschweißen der Komponenten zu unterbinden. Ein Schneidring aus Kupfer wird als Dichtring untergelegt und die Magnetrührkupplung mit dem Autoklavenkopf mit einem Maulschlüssel (SW32) verschraubt. Danach erfolgt die Montage des Propellers an der Rührwelle. Hierzu wird der Propeller auf die Welle aufgesetzt und mit einer Madenschraube an der abgeflachten Seite gesichert. Nun wird das Rührwerk in die entsprechende Halterung gesetzt und nur leicht befestigt, so dass ein Ausrichten noch möglich ist. Dann wird die flexible Welle in das Spannfutter des Rührwerks eingesetzt und auf den Vierkant an der Magnetrührkupplung gesteckt. Das Rührwerk wird jetzt mit der Welle gefluchtet, um einen möglichst ruhigen Lauf zu erreichen. Danach erfolgt die Montage des PFASchlauches an das Zwei-Wege-Nadelventil (N1). 


\section{Experimenteller Aufbau II}

\subsubsection{Montage des Tauchrohrs, des Thermoelements und der Berstscheibe}

In diesem Abschnitt werden die inneren Einbauten installiert. Das $6 \mathrm{~mm}$ Edelstahltauchrohr ist in sich zweiteilig verschraubt, da der obere Teil auf den abgeschrägten Flanken des Autoklavenkopfes sitzt und somit das Tauchrohr an sich eine Krümmung aufweist, die die Montage eines Einzelstücks unterbindet. Der Einschraubadapter (RZ1) wird mit einer Unterlegscheibe versehen und mit dem Autoklavenkopf verschraubt. Nun wird der obere Teil des Tauchrohrs von der Außenseite durch den Einschraubadapter (RZ1) geschoben, bis die Montage des unteren geraden Abschnitts des Tauchrohrs problemlos möglich ist. Hierbei ist darauf zu achten, dass an der Außenseite eine ausreichend große Restlänge des Tauchrohrs heraussteht, um die Montage des Zwei-Wege-Kugelhahns (K4) zu ermöglichen. Der obere Abschnitt wird dann mit dem Einschraubadapter (RZ1) fixiert. Nun können die beiden Abschnitte des Tauchrohres verschraubt werden und der PFA-Schlauch für die Einspeisung von 1,3Butadien an den Zwei-Wege-Kugelhahn (K4) angeschlossen werden. Dann wird der Einschraubadapter (RZ1) für das Thermoelement mit einer Unterlegscheibe am Autoklavenkopf befestigt. Das Thermoelement wird von außen vorsichtig durch den Einschraubadapter geschoben und mit ihm verschraubt. Als nächstes wird die Verbindung zwischen Thermoelement und Thermoregler hergestellt.

Die Berstscheibeneinrichtung ist bereits nach den Vorgaben aus Kapitel 5.2.8 komplettiert worden. Das 1/4“ NPT Gewinde wird mit Teflonband umwickelt und in die entsprechende Öffnung am Autoklavenkopf verschraubt. Eine Abdichtung ist nicht erforderlich, da das NPT Gewinde selbstdichtend ist.

\subsubsection{Montage des Manometers und des Ablassventils (K3)}

Unterhalb der Halteplatte werden für die seitlichen Öffnungen des Autoklavenkopfes ebenfalls G 1/4“ Einschraubadapter verwendet. Die Halteplatte des Autoklavenkopfes ist sehr groß dimensioniert worden, so dass ein $6 \mathrm{~mm}$ Edelstahlrohr als Verlängerung dient, um die Bedienbarkeit der Armaturen zu gewährleisten. Die Abdichtung der Einschraubadapter erfolgt wiederum mittels Unterlegscheiben. Das Manometer sollte im Sichtbereich des bedienenden Personals liegen. Der Drei-Wege-Kugelhahn (K3) dient als Ablassventil. Durch diesen Hahn kann der Stickstoffstrom und das restliche 1,3-Butadien reguliert bzw. verbrannt werden. Um ungefährliche Gase zu entsorgen, wird ein $6 \mathrm{~mm}$ PFA-Schlauch der entsprechenden Länge angeschlossen und in Richtung Abluft verlegt. 
Um die Verbrennung des Restmonomers besser kontrollieren zu können, wird in Schaltrichtung Flammrückschlagventil unmittelbar nach dem Drei-Wege-Kugelhahn (K3) das Zwei-Wege-Nadelventil (N2) geschaltet. Durch die bessere Dosierbarkeit des Nadelventils kann der Durchlass an 1,3-Butadien besser gesteuert und somit die Größe der Flamme in der Verbrennungskammer reguliert werden.

\subsubsection{Installation des Einschraubadapters (RZ3) und der Innenzelle}

Um die Montage des Einschraubadapters zu erleichtern, wird der Autoklavenbecher auf die Oberseite gestellt. Die Innenzelle wurde dabei bereits in den Innenraum geschoben und beim Umdrehen mit den Fingern fixiert. Der Außenkörper des Einschraubadapters RZ3 wird mit einer Unterlegscheibe aus Kupfer versehen und in das G 1/2“ geschraubt und mit einem Maulschlüssel (SW34) fest angezogen. Es ist darauf zu achten, dass an der Stirnseite des Einschraubadapters RZ3 zum Innenraum der Dichtring aus PTFE (vgl. Abbildung 5.2.4) sitzt. Dieser sorgt für einen sicheren Anstoß an die Innenzelle und verhindert somit das Auslaufen der Polymerisationslösung in den Druckkörper. Nun kann die hohle Zylinderkopfschraube mit Innensechskant in den Einschraubadapter RZ3 gesteckt und in das G 1/4“ Gewinde des Innenzylinders geschraubt werden. Im Anschluss wird ein G 3/8“ Einschraubadapter der Firma Swagelok ${ }^{\circledR}$ in den Adapter RZ3 geschraubt, wodurch der Ablass des Autoklavenbechers wieder auf das im experimentellen Aufbau übliche metrische System mit 6 mm kommt. An dieser Stelle können jetzt der Drei-WegeKugelhahn (K5) und die entsprechenden Kupplungen nach Abbildung 5.3.1 installiert werden.

\subsubsection{Montage des Autoklavenbechers}

Der Autoklavenbecher ist nun mit allen wichtigen Komponenten bestückt und kann jetzt unter den Autoklavenkopf installiert werden. Die acht M12 Zylinderkopfschrauben mit Innensechskant werden in die entsprechenden Bohrungen im Autoklavenkopf gesteckt. Nun kann der Autoklavenbecher vorsichtig von unten herangeführt werden. Die acht Schrauben werden mit einem Inbusschlüssel (SW10) angezogen. Es ist darauf zu achten, dass immer die gegenüberliegenden Schrauben nacheinander festgezogen werden, um ein Verkanten des Autoklavenbechers zu unterbinden. Die Apparatur ist anschließend auf Dichtigkeit zu prüfen. 


\section{Experimenteller Aufbau II}

Die Demontage der einzelnen Komponenten ist abschnittsweise und in umgekehrter Reihenfolge durchzuführen.

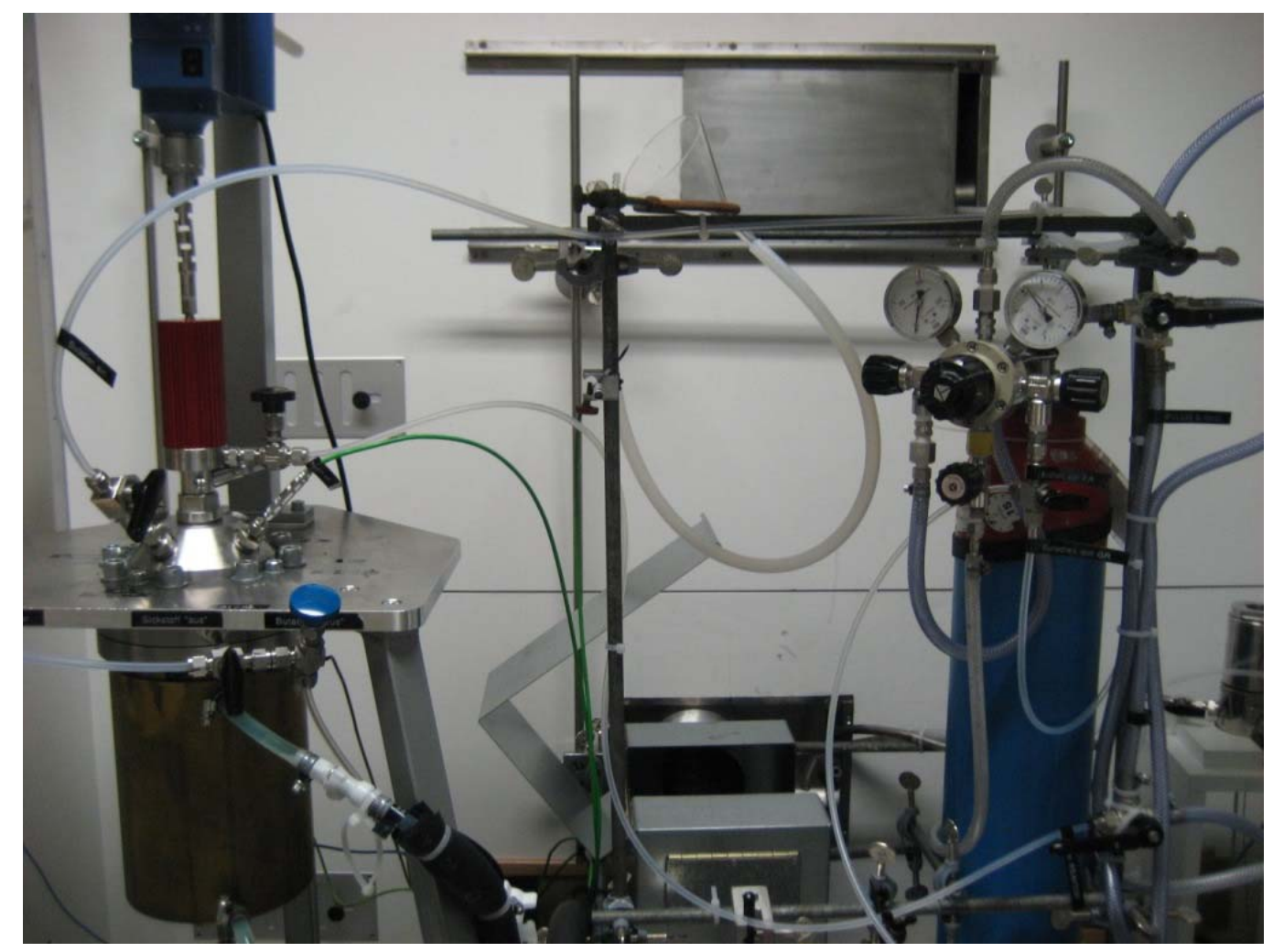

\section{Abbildung 5.3.2}

Fotografie des komplettierten experimentellen Aufbaus II: Im rechten Abschnitt des Bildes steht die Gasflasche mit 1,3-Butadien. In der Mitte ist die geöffnete Verbrennungskammer positioniert. Im linken Abschnitt ist der Autoklav II abgebildet. Im Vordergrund ist der DreiWege-Kugelhahn (K3) mit dem Zwei-Wege-Nadelventil (N2) gut zu erkennen. Weiterhin sind am Autoklavenbecher die Kühlschläuche angeschlossen und der Kühlmantel wird mit bläulichem Kühlmittel durchzogen. Am Autoklavenkopf ist die Magnetrührkupplung (MRK36) gut zu erkennen.

\subsection{Bedienung des Autoklaven II}

In diesem Kapitel soll die Inbetriebnahme des Autoklaven II beschrieben werden. Hierzu wird die effektivste und auch sicherste Arbeitsweise beschrieben, obwohl die Reihenfolge der einzelnen Arbeitsschritte auch variiert werden kann. 


\subsubsection{Abkühlen des Füllautoklaven und des Autoklavenbechers}

In diesem Arbeitsschritt werden die Vorbereitungen für die Einkondensation von 1,3Butadien in den Füllautoklaven und danach in den Autoklavenbecher getroffen. Zu diesem Zweck ist das Kältethermostat der Firma Lauda ${ }^{\circledR}$ eingeschaltet und die Temperatur auf $-20{ }^{\circ} \mathrm{C}$ eingestellt. Der Drei-Wege-Hahn (T1) wird so geschaltet, dass der Kühlkreislauf geschlossen ist. Nun wird in einem Dewargefäß aus Metall ein AcetonTrockeneisbad angesetzt, welches eine Temperatur von $-15{ }^{\circ} \mathrm{C}$ nicht überschreitet.

\subsubsection{Spülung des Füllautoklaven mit Stickstoff}

Am Flaschendruckminderer der Stickstoffflasche wird das Absperrventil (S1) geöffnet und über das Druckminderungsventil ein Reduzierdruck von 4 bar eingestellt. Der DreiWege-Hahn (H1) wird in Richtung (B2) geschaltet. Der Füllautoklav wird über die Kupplung des Typs QTM $^{\circledR}$ in das System integriert und in das Aceton-Trockeneisbad gestellt. Der Drei-Wege-Kugelhahn (K2) wird in Richtung Kupplung geöffnet. Nun kann die Stickstoffzufuhr (B2) und (B3) am Flaschendruckminderer für Sondergase geöffnet und der Drei-Wege-Kugelhahn (K1) in Richtung Füllautoklav geschaltet werden. Der Stickstoffstrom gelangt in den Füllautoklaven. Durch mehrmaliges Betätigen des DreiWege-Kugelhahns (K2) kann der Füllautoklav gespült werden. Nach Beendigung des Spülvorganges wird das Absperrventil (S1) geschlossen und das gesamte System einschließlich Füllautoklav mit dem Ablassventil (B4) auf Umgebungsdruck gebracht. Im Anschluss werden die Ventile (B2) und (B4) geschlossen.

\subsubsection{Einkondensation von 1,3-Butadien (BD) in den Füllautoklaven}

Es ist zu kontrollieren, dass die Drei-Wege-Hähne (K1) und (K2) richtig geschaltet sind. Weiterhin ist darauf zu achten, dass die Ventile (B2) und (B4) geschlossen sind, bevor eine Einkondensation von 1,3-Butadien in den Füllautoklaven ermöglicht wird. Erst jetzt wird das Absperrventil (B1) an der Butadiengasflasche geöffnet und das Monomer kondensiert in den vortemperierten Füllautoklaven. Durch mehrmaliges Betätigen des Drei-Wege-Kugelhahns (K2) kann der Überdruck der Stickstoffatmosphäre, welcher während der Einkondensation entsteht, reduziert werden. Nach Beendigung der Einkondensation werden der Drei-Wege-Kugelhahn (K2) und die Absperrventile (B1) und (B3) geschlossen. Um Korrosion am Flaschendruckminderer für Sondergase zu verhindern, erfolgt ein Spülvorgang mit Stickstoff. Aus diesem Grund wird der Drei- 


\section{Experimenteller Aufbau II}

Wege-Kugelhahn (K1) geschlossen bzw. in Richtung des Autoklaven II geschaltet. Die Absperrventile (S1) und (B1) werden geöffnet und der Flaschendruckminderer für Sondergase mit 4 bar Stickstoff geflutet. Durch mehrmaliges Betätigen des Ablassventils (B4) kann der Spülvorgang wiederholt werden. Zum Schluss wird der Drei-Wege-Hahn

(H1) in Richtung (H2) geschaltet. Nun kann der Flaschendruckminderer auf Umgebungsdruck gebracht werden. Vorsichtshalber ist das Absperrventil (S1) beim längeren Nichtgebrauch des Stickstoffstroms zu schließen.

\subsubsection{Vorbereitung der Polymerisation}

Der Einfüllstopfen am Autoklavenkopf wird entfernt und der Drei-Wege-Kugelhahn (K3) in Richtung Olive geschaltet (Abbildung 5.3.1), um einen Druckausgleich zu ermöglichen. Nun können über einen Trichter die einzelnen Komponenten des Polymerisationsansatzes in den gekühlten Autoklavenbecher vorgelegt werden. Das Rührwerk wird auf die gewünschte Drehzahl gebracht. Das Gewinde des Einfüllstopfens wird mit Teflonspray besprüht. Danach wird die Einfüllöffnung wieder verschlossen und der Drei-Wege-Kugelhahn (K3) geschlossen.

\subsubsection{Spülung des Autolaven II mit Stickstoff}

Die Drei-Wege-Kugelhähne (H1) und (H2) werden in Richtung Zwei-Wege-Nadelventil (N1) geschaltet. Nachdem kontrolliert wurde, dass alle Ventile und Hähne am Autoklavenbecher geschlossen sind, kann das Absperrventil (S1) geöffnet und ein Reduzierdruck von 4 bar eingestellt werden. Durch das Öffnen des Zwei-WegeNadelventils (N2) kann der Reaktionsraum mit Stickstoff geflutet werden. Zum Ablassen des Überdruckes wird der Drei-Wege-Kugelhahn (K3) vorsichtig in Richtung Olive geöffnet, nachdem das Zwei-Wege-Nadelventil geschlossen worden ist. Dieser Spülzyklus wird dreimal wiederholt. Das Absperrventil (S1) wird geschlossen. Nun wird ein konstanter Stickstoffstrom eingestellt, um die Schläuche und den Flaschendruckminderer an der Stickstoffflasche auf Umgebungsdruck zu bringen. Dazu wird der Drei-Wege-Kugelhahn (K3) wieder in Richtung Olive geschaltet und das ZweiWege-Nadelventil vorsichtig geöffnet. Der Druckverlust kann an den Anzeigen für den Gesamtdruck und den Reduzierdruck am Flaschendruckminderer verfolgt werden. Nach Druckausgleich werden alle verwendeten Hähne und Ventile geschlossen. Somit kann im Autoklavenbecher eine Stickstoffatmosphäre mit Umgebungsdruck hergestellt werden. 


\subsubsection{Einkondensation von 1,3-Butadien (BD) in den Autoklaven II}

Nachdem alle Komponenten des Polymerisationsansatzes bereits in den gekühlten Autoklavenbecher vorgelegt worden sind, wird zum Abschluss 1,3-Butadien vom Füllautoklaven in den Reaktionsraum überführt. Um die Einkondensation zu erleichtern wird ein Wasserbad auf $T=80^{\circ} \mathrm{C}$ erhitzt. Der Füllautoklav wird über die Kupplung $\mathrm{QTM}^{\circledR}$ wieder in das System integriert und der Drei-Wege-Kugelhahn (K1) in Richtung Zwei-Wege-Kugelhahn (K4) geschaltet. Nachdem der Füllautoklav in das Wasserbad gestellt wurde, kann der Drei-Wege-Kugelhahn (K2) in Richtung Kupplung geöffnet werden. Nun wird der Zwei-Wege-Hahn (K4) am Autoklaven II geöffnet. Durch das Verdampfen des 1,3-Butadiens kühlt sich der Füllautoklav ab, so dass nicht mit einem Durchgang das komplette Monomer in den Reaktionsraum des Autoklaven II überführt werden kann. In diesem Fall werden die Hähne (K2) und (K4) geschlossen und der Temperaturausgleich zwischen Wasserbad und Füllautoklav abgewartet. Anschließend kann das restliche 1,3-Butadien in der eben beschriebenen Weise einkondensiert werden. Die exakte Masse an Monomer wird durch Differenzwägung des Füllautoklaven bestimmt.

\subsubsection{Starten der Polymerisation}

Vor dem Starten der Heizphase muss der Kühlmantel entleert werden, da sonst der Druck der Kühlflüssigkeit durch die steigende Temperatur zu groß werden würde. Das Thermostat wird ausgeschaltet. In die freie Öffnung des Drei-Wege-Hahns (T1) wird ein Gummigebläse gesteckt. Der Hahn (T1) wird so geschaltet, dass der ursprüngliche Kühlkreislauf unterbrochen ist. Nun kann durch Betätigen des Gummigebläses die Kühlflüssigkeit in das Reservoir des Thermostaten gedrückt werden. Nachdem der Kühlmantel luftleer gepumpt worden ist, kann am Temperaturregler die gewünschte Polymerisationstemperatur eingestellt werden. Nachdem der Sollwert der Reaktionstemperatur ohne Zwischenfälle erreicht wurde, verläuft die Polymerisation stabil und der Autoklav arbeitet autark.

\subsubsection{Probenentnahme während der Polymerisation}

Die Probenentnahme kann beim Autoklaven II direkt innerhalb des geschlossenen Systems durchgeführt werden. Dazu wird ein Probennehmer über die Kupplungen 1 und 2 des Typs $\mathrm{QF}^{\circledR}$ in das System integriert. Der Zwei-Wege-Kugelhahn (P2) am 


\section{Experimenteller Aufbau II}

Probennehmer ist geschlossen und der Drei-Wege-Kugelhahn (P1) wird in Richtung Kupplung 2 geschaltet. Nun wird der Drei-Wege-Kugelhahn (K5) betätigt, um eine Teil der Polymerisationslösung in den Probennehmer zu überführen. Nach Beendigung der Probenentnahme wird der Drei-Wege-Kugelhahn (K5) in Richtung Olive geschaltet, um die Kupplungen 1 und 2 druckfrei zu bekommen. Danach kann der Probennehmer abgekuppelt werden und der Umsatz gravimetrisch bestimmt werden. Zur Entleerung des Probennehmers wird der Drei-Wege-Kugelhahn (P1) in Richtung Olive geschaltet und der Zwei-Wege-Kugelhahn (P2) geöffnet. Nachdem alle Proben entnommen worden sind, wird die Kupplung 1 geöffnet. Dadurch wird die Entnahme des gesamten Polymerisationsansatzes erleichtert, da dieser durch das Kupplungsgegenstück fließen kann.

\subsubsection{Beenden der Polymerisation}

Eine Stunde vor Erreichen der Polymerisationsdauer wird das Kältethermostat der Firma Lauda ${ }^{\circledR}$ eingeschaltet und die Temperatur auf $-20{ }^{\circ} \mathrm{C}$ eingestellt. Es ist darauf zu achten, dass die Umwälzpumpe des Thermostaten noch nicht eingeschaltet ist. Am Ende der Polymerisation wird der Temperaturregler auf $0{ }^{\circ} \mathrm{C}$ gestellt und der Drei-Wege-Hahn (T1) so geschaltet, dass der Kühlkreislauf geschlossen ist. Nun wird die Umwälzpumpe des Thermostaten aktiviert und der Kühlmantel mit Kühlflüssigkeit durchströmt. Am Temperaturregler kann der Abfall der Temperatur im Inneren des Autoklaven II verfolgt werden. Nachdem ein Temperaturbereich zwischen $20{ }^{\circ} \mathrm{C}$ und $30{ }^{\circ} \mathrm{C}$ innerhalb von 15 Minuten erreicht ist, kann das Kältethermostat abgeschaltet werden.

\subsubsection{Entsorgung von Restmonomer}

Nachdem die Polymerisationslösung im Inneren des Autoklavenbechers abgekühlt ist, kann mit der Entsorgung des restlichen 1,3-Butadiens begonnen werden. Dazu wird der Gasbrenner angezündet und danach die Verbrennungskammer geschlossen. Es ist zu kontrollieren, dass das Zwei-Wege-Nadelventil (N2) geschlossen ist. Der Drei-WegeKugelhahn (K3) wird in Richtung (N2) geschaltet. Nun kann der Durchfluss über das Zwei-Wege-Nadelventil und somit die Höhe der Verbrennungsflamme reguliert werden. Nach Beendigung der Entsorgung werden alle Hähne und Ventile geschlossen und der Gasbrenner in der Verbrennungskammer ausgeschaltet. 


\subsubsection{Entnahme der Polymerisationslösung}

Um den gesamten Polymerisationsansatz aus dem Autoklavenbecher so entnehmen, muss der Drei-Wege-Kugelhahn (K3) in Richtung Olive geschaltet werden. Ein entsprechendes Gefäß wird nun unter dem Gegenstück der Kupplung 1 platziert und durch Öffnen des Drei-Wege-Kugelhahnes (K5) kann der Inhalt des Autoklavenbechers abgelassen werden. Manchmal wird ein leichter Überdruck im Autoklavenbecher benötigt, um teilweise höherviskose Lösungen ablaufen zu lassen. In diesem Fall bleibt der Drei-WegeKugelhahn (K3) geschlossen. Nun wird Stickstoff über das Zwei-Wege-Nadelventil (N2) in den Autoklavenbecher geleitet (siehe Kapitel 5.4.5). Es ist zwingend darauf zu achten, dass der eingestellte Reduzierdruck am Flaschendruckminderer der Stickstoffflasche nicht über 2 bar steigt. Nur so kann ein ruhiges Ablaufen der Polymerisationslösung gewährleistet werden. 


\section{$\underline{\text { 6. Experimentelles }}$}

In diesem Kapitel werden die verwendeten Chemikalien und die Synthesen der RAFTAgenzien beschrieben. Weiterhin werden die Bezeichnungen und technischen Spezifikationen der Analysengeräte benannt.

\subsection{Verwendete Chemikalien}

\subsubsection{Lösungsmittel}

Die Lösungsmittel Toluol, Dimethylsulfoxid, Dibenzylether, Chloroform, Dichlormethan, Methanol, Dimethylacetamid, Tetrahydrofuran, Essigester und Hexan werden als p.a. ohne weitere Aufarbeitung direkt eingesetzt. Destilliertes Wasser wird aus dem hauseigenen Kreislauf entnommen.

\subsubsection{Monomere}

Butylacrylat<smiles>C=CC(=O)OCCCC</smiles>

Butylacrylat (BA, $M=128,17 \mathrm{~g} / \mathrm{mol}$, Sigma Aldrich, $\geq 99 \%$, stabilisiert mit 4-Methoxyphenol) wird vor der Verwendung durch Säulenchromatographie über basischen Aluminiumoxid (Fluka, aktiviert, Typ 5016 basisch) vom Inhibitor befreit.

Styrol

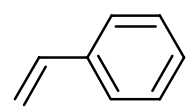

Styrol (St, $M=104,15 \mathrm{~g} / \mathrm{mol}$, Sigma Aldrich, ReagentPlus ${ }^{\circledR}, \geq 99 \%$, stabilisiert mit 4-tert-Butylcatechol) wird vor der Verwendung durch Säulenchromatographie über basischen Aluminiumoxid (Fluka, aktiviert, Typ 5016 basisch) vom Inhibitor befreit. 
1,3-Butadien

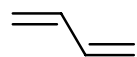

1,3-Butadien N25 (BD, $M=54,09 \mathrm{~g} / \mathrm{mol}$, Air Liquide, $\geq 99,5$ Vol.\%, stabilisiert) wird ohne weitere Reinigung eingesetzt.

Isopren

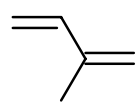

Isopren (IP, $M=68,12 \mathrm{~g} / \mathrm{mol}$, Fluka, $\geq 98 \%$, stabilisiert mit 4-tert-Butylcatechol) wird vor der Verwendung unter reduziertem Druck destilliert.

\subsubsection{Initiatoren}

$1,1^{\prime}$-Azobis(cyclohexanecarbonitril)<smiles>N#CC1(N=NC2(C#N)CCCCC2)CCCCC1</smiles>

1,1'-Azobis(cyclohexanecarbonitril) (ACC, $M=244,34 \mathrm{~g} / \mathrm{mol}$, Sigma Aldrich, $\geq 98 \%$ ) wird ohne weitere Reinigung eingesetzt.

\subsubsection{Andere Chemikalien}

Alle kommerziell erhältlichen Substanzen und Gemische werden in der höchsten Reinheit bestellt und ohne weitere Aufarbeitung verwendet. Firmen und Hersteller, von denen die Chemikalien bezogen werden: Sigma Aldrich, Merck, ABCR, Fluka, VWR und Wako Chemicals.

Die verwendeten Industrieruße werden von der Continental Reifen GmbH zur Verfügung gestellt. Vor der weiteren Verwendung wird der Ruß für $t=24 \mathrm{~h}$ im Soxhlet mit destilliertem Wasser gewaschen und dann im Hochvakuum für weitere 24 Stunden bei $T=100{ }^{\circ} \mathrm{C}$ getrocknet. 


\subsection{Synthese der RAFT-Agenzien}

In diesem Kapitel werden die Synthesen der verwendeten RAFT-Agenzien und der Ankergruppen für die Polymerisation von Oberflächen vorgestellt.

\subsubsection{Bis(1-phenylethyl)trithiocarbonat (BPETC)}<smiles>CC(SC(=S)SC(C)c1ccccc1)c1ccccc1</smiles>

Ein Gemisch aus Schwefelkohlenstoff (1,90 g; 0,025 mol) und Caesiumcarbonat (8,14 g; $0,025 \mathrm{~mol})$ in $\mathrm{N}, \mathrm{N}$-Dimethylacetamid $(20 \mathrm{ml})$ wird für 20 Minuten bei Raumtemperatur gerührt. Es kommt zu einer intensiven Rotfärbung der Reaktionsmischung.

Es erfolgt die langsame Zugabe von 1-Bromo-ethylbenzol (4,62 g; 0,025 mol) in $\mathrm{N}, \mathrm{N}$ Dimethylacetamid (5 ml). Die Reaktionslösung wird für 24 Stunden bei Raumtemperatur gerührt und danach in Eiswasser $(150 \mathrm{ml})$ überführt. Die wässrige Phase wird mit Essigester (3 mal $50 \mathrm{ml}$ ) extrahiert. Die vereinigten organischen Phasen werden über Natriumsulfat getrocknet und das Lösungsmittel im Hochvakuum entfernt. Das gewünschte Produkt wird als gelbliches Öl (7,79 g) in einer Ausbeute von $98 \%$ erhalten.

${ }^{1} \mathbf{H}-\mathbf{N M R}\left(300 \mathrm{MHz}, \mathrm{CDCl}_{3}\right) \delta(\mathrm{ppm}): 1,8\left(\mathrm{~d}, 6 \mathrm{H}, 2 \times \mathrm{CH}_{3}\right) ; 5,3(\mathrm{q}, 2 \mathrm{H}, 2 \times \mathrm{CH}) ; 7,3-7,4$ $\left(\mathrm{m}, 10 \mathrm{H}, 2 \times \mathrm{C}_{6} \mathrm{H}_{5}\right)$

${ }^{13}$ C-NMR (75,5 MHz, $\left.\mathrm{CDCl}_{3}\right) \delta(\mathrm{ppm}): 21,3\left(2 \mathrm{x} \mathrm{CH}_{3}\right) ; 50,0(2 \times \mathrm{CH}) ; 127,0-128,6$ (10 x CH); 141,7 (2 x C); 221,5 (C=S)

\subsubsection{Furfuryl-2-bromopropanoat (FABP)}<smiles>CC(Br)C(=O)OCc1ccco1</smiles>

$\mathrm{Zu}$ einer eisgekühlten Lösung von 2-Furylmethanol (5,10 g; 0,052 mol) in $70 \mathrm{ml}$ Chloroform wird Triethylamin (5,26 g; 0,052 mol) hinzugegeben. Danach erfolgt die langsame Zugabe von 2-Bromopropionylbromid (9,93 g; 0,046 mol) in $10 \mathrm{ml}$ 
Chloroform. Die Reaktionsmischung wird für 24 h bei RT gerührt. Zur Aufarbeitung wird das Reaktionsgemisch mit Wasser (1 mal $80 \mathrm{ml}), 10$ \%iger Schwefelsäure (1 mal $80 \mathrm{ml})$ und mit gesättigter $\mathrm{NaHCO}_{3}$-Lsg. (2 mal $50 \mathrm{ml}$ ) gewaschen. Die organische Phase wird über Magnesiumsulfat getrocknet, eingeengt und im Hochvakuum getrocknet. Die Ausbeute beträgt $67 \%$.

${ }^{1} \mathbf{H}-\mathbf{N M R}\left(300 \mathrm{MHz}, \mathrm{CDCl}_{3}\right) \delta(\mathrm{ppm}): 1,8\left(\mathrm{~d}, 3 \mathrm{H}, \mathrm{CH}_{3}\right) ; 4,3(\mathrm{q}, 1 \mathrm{H}, \mathrm{CH}) ; 5,2(\mathrm{~s}, 2 \mathrm{H}$, $\left.\mathrm{CH}_{2}\right) ; 6,3-6,4(\mathrm{~m}, 2 \mathrm{H}, 2 \times \mathrm{CH}) ; 7,4(\mathrm{~d}, 1 \mathrm{H}, \mathrm{CH})$

${ }^{13}$ C-NMR (75,5 MHz, $\left.\mathrm{CDCl}_{3}\right) \delta(\mathrm{ppm}): 21,8\left(\mathrm{CH}_{3}\right) ; 40,0(\mathrm{CH}) ; 59,6\left(\mathrm{CH}_{2}\right) ; 110,9(\mathrm{CH})$; $111,5(\mathrm{CH}) ; 143,8(\mathrm{CH}) ; 148,8(\mathrm{C}) ; 170,1(\mathrm{C}=\mathrm{O})$

\subsubsection{Furfuryl-2-bromopropanthioat (FTBP)}<smiles>CC(Br)C(=O)SCc1ccco1</smiles>

$\mathrm{Zu}$ einer eisgekühlten Lösung von 2-Furylmethanthiol $(5,94 \mathrm{~g}, 0,052 \mathrm{~mol})$ in $70 \mathrm{ml}$ Chloroform wird Triethylamin (5,26 g; 0,052 mol) hinzugegeben. Danach erfolgt die langsame Zugabe von 2-Bromopropionylbromid (9,93 g; 0,046 mol) in $10 \mathrm{ml}$ Chloroform. Die Reaktionsmischung wird für $24 \mathrm{~h}$ bei RT gerührt. Zur Aufarbeitung wird das Reaktionsgemisch mit Wasser (1 mal $80 \mathrm{ml}), 10$ \%iger Schwefelsäure (1 mal $80 \mathrm{ml})$ und mit gesättigter $\mathrm{NaHCO}_{3}$-Lsg. (2 mal $50 \mathrm{ml}$ ) gewaschen. Die organische Phase wird über Magnesiumsulfat getrocknet, eingeengt und im Hochvakuum getrocknet. Die Ausbeute beträgt $65 \%$.

${ }^{1} \mathbf{H}-\mathrm{NMR}\left(300 \mathrm{MHz}, \mathrm{CDCl}_{3}\right) \delta(\mathrm{ppm}): 1,8\left(\mathrm{~d}, 3 \mathrm{H}, \mathrm{CH}_{3}\right) ; 4,2\left(\mathrm{~s}, 2 \mathrm{H}, \mathrm{CH}_{2}\right) ; 4,5(\mathrm{q}, 1 \mathrm{H}$, $\mathrm{CH}) ; 6,3-6,4(\mathrm{~m}, 2 \mathrm{H}, 2 \times \mathrm{CH}) ; 7,4(\mathrm{~d}, 1 \mathrm{H}, \mathrm{CH})$

${ }^{13}$ C-NMR (75,5 MHz, $\left.\mathrm{CDCl}_{3}\right) \delta(\mathrm{ppm}): 22,0\left(\mathrm{CH}_{3}\right) ; 26,6\left(\mathrm{CH}_{2}\right) ; 47,8(\mathrm{CH}) ; 108,4(\mathrm{CH})$; $110,6(\mathrm{CH}) ; 142,4(\mathrm{CH}) ; 149,4(\mathrm{C}) ; 195,4(\mathrm{C}=\mathrm{O})$ 


\subsubsection{Natrium-(propyl)-trithiocarbonat (TTCP)}

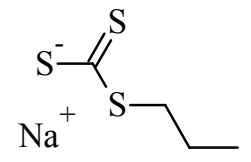

$\mathrm{Zu}$ einer eisgekühlten Lösung von Propanthiol (4,00 g; 0,053 mol) in $50 \mathrm{ml}$ Tetrahydrofuran erfolgt die Zugabe von 4,20 g 50 \%iger Natriumhydroxidlösung. Danach erfolgt die langsame Zugabe von $\mathrm{CS}_{2}(4,00 \mathrm{~g} ; 0,053 \mathrm{~mol})$. Dabei ist darauf zu achten, dass die Temperatur $10{ }^{\circ} \mathrm{C}$ nicht übersteigt. Die Reaktionsmischung wird für $24 \mathrm{~h}$ bei Raumtemperatur gerührt und aus Hexan umkristallisiert. Die Ausbeute beträgt 64 \%.

${ }^{1} \mathbf{H}-\mathrm{NMR}\left(300 \mathrm{MHz}\right.$, Aceton-D6) $\delta$ (ppm): 0,9 (t, 3H, $\left.\mathrm{CH}_{3}\right) ; 1,6\left(\mathrm{sext}, 2 \mathrm{H}, \mathrm{CH}_{2}\right) ; 3,0$ (t, $\left.2 \mathrm{H}, \mathrm{CH}_{2}\right)$

${ }^{13}$ C-NMR (75,5 MHz, Aceton-D6) $\delta(\mathrm{ppm}): 13,7\left(\mathrm{CH}_{3}\right), 21,8\left(\mathrm{CH}_{2}\right), 38,9\left(\mathrm{CH}_{2}\right)$, $210,0(\mathrm{C}=\mathrm{S})$

\subsubsection{Propyl-(furfurylpropanioat)-trithiocarbonat (FABPTTCP)}<smiles>CCCSC(=S)SC(C)C(=O)OCc1ccco1</smiles>

$\mathrm{Zu}$ einer Lösung von Furfuryl-2-bromopropanoat (1,49 g; 0,0064 mol) in DMSO (25 ml) wird eine Lösung von Natrium-(propyl)-trithiocarbonat (1,12 g; 0,0064 mol) in $10 \mathrm{ml}$ DMSO hinzugegeben. Das Reaktionsgemisch wird bei RT für $48 \mathrm{~h}$ gerührt und danach mit Diethylether (3 mal $20 \mathrm{ml}$ ) gewaschen. Die vereinigten organischen Phasen werden mit Wasser gewaschen und über Natriumsulfat getrocknet. Das Lösungsmittel wird im Hochvakuum verdampft und das Produkt wird mit einer Ausbeute von $72 \%$ erhalten.

${ }^{\mathbf{1}} \mathbf{H}$-NMR $\left(300 \mathrm{MHz}, \mathrm{CDCl}_{3}\right) \delta(\mathrm{ppm}): 1,0\left(\mathrm{t}, 3 \mathrm{H}, \mathrm{CH}_{3}\right) ; 1,5$ (d, 3H, $\left.\mathrm{CH}_{3}\right) ; 1,6$ (quin., 2H, $\left.\mathrm{CH}_{2}\right) ; 3,3$ (t, 2H, $\left.\mathrm{CH}_{2}\right) ; 4,8$ (q, 1H, CH); 5,1 (s, 2H, $\left.\mathrm{CH}_{2}\right) ; 6,3-6,4$ (m, 2H, 2 x CH); 7,4 (d, $1 \mathrm{H}, \mathrm{CH})$ 
${ }^{13}$ C-NMR (75,5 MHz, $\left.\mathrm{CDCl}_{3}\right) \delta(\mathrm{ppm}): 13,4\left(\mathrm{CH}_{3}\right) ; 16,8\left(\mathrm{CH}_{3}\right) ; 21,4\left(\mathrm{CH}_{2}\right) ; 39,0\left(\mathrm{CH}_{2}\right)$; 47,9 (CH); 59,2 $\left(\mathrm{CH}_{2}\right) ; 110,6(\mathrm{CH}) ; 111,0(\mathrm{CH}) ; 143,5(\mathrm{CH}) ; 148,9(\mathrm{C}) ; 170,8(\mathrm{C}=\mathrm{O})$; $221,8(\mathrm{C}=\mathrm{S})$

\subsubsection{Cumyldithiobenzoat (CDB)}

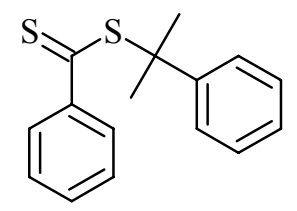

$\mathrm{Zu}$ einer Suspension aus einer 30\%igen Lösung aus Natriummethanolat in Methanol (36 g), elementarem Schwefel (6,4 g, 0,2 mol) und Methanol (30 ml) wird innerhalb einer Stunde Benzylchlorid (12,6g, 0,1 mol) tropfenweise hinzugegeben. Es entsteht eine leichte Wärmetönung. Die entstandene braune Lösung wird dann über Nacht bei $80{ }^{\circ} \mathrm{C}$ unter Rückfluss gerührt.

Nach dem Abkühlen auf Raumtemperatur wird der weiße Feststoff abfiltriert und das Lösungsmittel im Rotationsverdampfer entfernt. Der braune Feststoff wird mit destilliertem Wasser $(100 \mathrm{ml})$ aufgenommen und mit Diethylether $(3 \times 50 \mathrm{ml})$ gewaschen. Danach wird nochmals Diethylether $(80 \mathrm{ml})$ hinzu gegeben und das Zweiphasensystem mit $37 \% \mathrm{HCl}$ angesäuert, bis es zur Entfärbung der wässrigen Phase kommt. Die vereinigten organischen Phasen werden über Calciumchlorid getrocknet. Nach Entfernen des restlichen Ethers unter vermindertem Druck bleibt die Dithiobenzoesäure als dunkles purpurfarbenes Öl zurück. Das Öl wird in n-Hexan $(10 \mathrm{ml})$ aufgenommen und mit $\alpha$ Methylstyrol (20\%iger Überschuss) vermengt. Nach Zugabe einer geringen Menge des Säurekatalysators para-Toloulsulfonsäure (1\%) wird die Lösung über Nacht unter Rückfluss gerührt. Das Rohprodukt wird säulenchromatographisch gereinigt (Pentan).

${ }^{1} \mathbf{H}-\mathbf{N M R}\left(300 \mathrm{MHz} ; \mathrm{CDCl}_{3}\right) \delta(\mathrm{ppm}): 2,0\left(\mathrm{~s}, 6 \mathrm{H}, 2 \times \mathrm{CH}_{3}\right) ; 7,3-7,4\left(\mathrm{~m}, 10 \mathrm{H}, 2 \times \mathrm{C}_{6} \mathrm{H}_{5}\right)$ 


\subsection{Charakterisierung und Analytik}

\subsubsection{GPC-Analyse}

Für die Bestimmung der mittleren Molekulargewichte und der Molekulargewichtsverteilung von Polymeren wird häufig die Gel-PermationsChromatographie (GPC) oder auch Größenausschluss-Chromatographie (SEC) verwendet. Die Trennung des Polymers erfolgt hierbei nach dem Ausschlussprinzip und richtet sich nach der Kettenlänge. Als Detektionssystem dient ein Differentialrefraktometer (RI) für die Bestimmung des Brechungsindex und ein UVSpektrometer (UV). Bei dem RI-Detektor ist die Intensität des gemessenen Signals proportional zum Massengehalt des Polymers in der mobilen Phase. Der UV-Detektor hingegen kann auf verschiedene Wellenlängen eingestellt werden, bei denen die Absorbanz erfolgen kann. Aus diesem Grund kann zwischen UV-aktiven Endgruppen der RAFT-Polymerisation und zum Beispiel UV-aktiven Monomereinheiten unterschieden werden. Je nach eingestellter Wellenlänger beschreibt die Intensität des UV-Signals also die Anzahl der Polymerketten oder eben wieder die Massenkonzentration des Polymers in der mobilen Phase.

Die GPC-Analysen werden mit einer HPLC-Anlage durchgeführt, welche aus einer Waters 515 HPLC-Pumpe, einem JASCO AS-2055 Autosampler, einem Detektorensystem, einer Vorsäule und drei Trennsäulen besteht (PSS SDV, 8 x $50 \mathrm{~mm}$, $5 \mu$; PSS SDV, 8 x 300 mm, $5 \mu, 10^{5} \AA$; PSS SDV, 8 x 300 mm, $5 \mu, 100 \AA ̊$; PSS SDV, $8 \times 300 \mathrm{~mm}, 5 \mu, 1000 \AA$ ). Die Detektion der Fraktionen erfolgt an einem RI- (Waters 2410) und einem UV/vis-Detektor (Viskotek VE3210). Die Messungen erfolgen bei $T=35^{\circ} \mathrm{C}$ mit $\mathrm{THF}$ als Eluationsmittel mit einer Flussgeschwindigkeit von $1,0 \mathrm{~cm}^{3} / \mathrm{min}$. Die Konzentration des Polymers sollte bei $3 \mathrm{mg} / \mathrm{cm}^{3}$ liegen. Die Eichung wird mit engverteilten Polystyrol- bzw. Polybutadienstandards $\left(M_{\mathrm{n}}=400\right.$ bis $\left.3 \cdot 10^{6} \mathrm{~g} / \mathrm{mol}\right)$ der Firma PSS durchgeführt. Als interner Standard wird Toluol verwendet. Die Auswertung erfolgt mit dem Programm PSS WINGPC 6.20.

\subsubsection{NMR-Spektroskopie}

Die ${ }^{1}$ H-NMR-Spektroskopie wird an den Modellen Unity 300 (300 MHz) der Firma $\operatorname{Varian}^{\circledR}$ und AMX-300 (300 MHz) der Firma Bruker ${ }^{\circledR}$ durchgeführt. Als Lösungsmittel dient $\mathrm{CDCL}_{3}$ und Aceton-D6. Als Signalreferenz dient Tetramethylsilan und die chemische Verschiebung wird in ppm angegeben. 
Die ${ }^{13} \mathrm{C}$-NMR-Spektroskopie wird an den Modellen Unity $300(75,5 \mathrm{MHz})$ der Firma $\operatorname{Varian}^{\circledR}$ und AMX-300 (75,5 MHz) der Firma Bruker ${ }^{\circledR}$ durchgeführt. Als Lösungsmittel dient $\mathrm{CDCL}_{3}$ und Aceton-D6. Als Signalreferenz dient Tetramethylsilan und die chemische Verschiebung wird in ppm angegeben.

\subsubsection{Elementaranalyse und Thermogravimetrie}

Elementaranalysen für Stickstoff, Kohlenstoff, Wasserstoff und Schwefel werden durch das Analysenlabor des Instituts für Anorganische Chemie der Universität Göttingen durchgeführt. Die Bestimmung erfolgt an dem Gerät Elementar Vario EL III.

Die Thermogravimetrie (TGA) wird mit einem Gerät der Firma Netzsch ${ }^{\circledR}$ vom Typ STA 409 PC untersucht. Die Proben werden mit einer Aufheizrate von $10 \mathrm{~K} / \mathrm{min}$, beginnend bei Raumtemperatur bis $T=800{ }^{\circ} \mathrm{C}$, gemessen. Die Messungen erfolgen unter Inertgasatmosphäre mit einem Stickstoffstrom von $30 \mathrm{ml} / \mathrm{min}$.

\subsection{Durchführung der Polymerisationen}

In diesem Abschnitt wird der experimentelle Ablauf der Polymerisationen beschrieben, welche nicht in den experimentellen Aufbauten I oder II (Kapitel 4 und 5) durchgeführt wurden. Die Experimente, welche für die Polymerisationen an Industrierußen durchgeführt werden (Kapitel 8), verlaufen unter verschiedenen Reaktionsbedingungen (z.B. unter Luftsauerstoff). Diese werden in den jeweiligen Kapiteln beschrieben. Der Polymerisationsansatz wird in einem entsprechenden Glaskolben einer Analysenwaage der Firma Sartorius ${ }^{\circledR}$ (Analytic AC $210 \mathrm{~S}$ ) ein- bzw. abgewogen. Die Polymerisationstemperatur beträgt einheitlich $T=87^{\circ} \mathrm{C}$. An dieser Stelle wird die Durchführung der Polymerisation beschrieben. Es sind dabei zwei Varianten zu unterscheiden.

\subsubsection{Polymerisationen im Thermoblock (Variante 1)}

Die Reaktionsmischung wird gleichmäßig auf Schraubdeckelgläser aufgeteilt und mit den gasundurchlässigen Deckeln verschlossen. Die Polymerisationen werden in einem Thermoblock der Firma Kleinfeld ${ }^{\circledR}$ (MBT 250) durchgeführt, welcher auf einem Schüttler der Firma Edmund Bühler ${ }^{\circledR}$ (KS-15) befestigt ist. Der Thermoblock wird auf die gewünschte Polymerisationstemperatur eingestellt. Zum Beenden der Polymerisation wird die Probe zum Abkühlen in Eiswasser gestellt. Die Umsatzbestimmung erfolgt gravimetrisch. 
Experimentelles

\subsubsection{Polymerisationen im Ölbad (Variante 2)}

Die Reaktionsmischung wird gleichmäßig auf Schraubdeckelgläser, welche einen Rührfisch beinhalten, aufgeteilt. Die Probengläser werden mit gasundurchlässigen Deckeln verschlossen. Die Polymerisationen erfolgt in einem temperierten Ölbad, welches auf einer Heizplatte mit Rührfunktion und Temperaturfühler steht. Zum Beenden der Polymerisation wird die Probe zum Abkühlen in Eiswasser gestellt. Die Umsatzbestimmung erfolgt gravimetrisch. 


\section{Auswertung der statistischen Copolymerisation}

In diesem Kapitel werden die statistischen Copolymerisation von 1,3-Butadien mit verschiedenen Monomeren und unterschiedlichen RAFT-Agenzien ausgewertet. Die Polymerisationen werden im experimentellen Aufbau I (Kapitel 4) realisiert, wobei keine Probenentnahme erfolgte. Aus diesem Grund werden vermehrt die Molekulargewichtsverteilungen der einzelnen Proben ausgewertet, da für eine aussagekräftige Entwicklung der mittleren Molekulargewichte mit dem Umsatz die Datenmenge zu gering ist. Durch den Vergleich der Molekulargewichtsverteilung des Massendetektors (RI) und des UV-Detektors (UV) der GPC kann allerdings auch die Reaktionskontrolle der RAFT-Agenzien qualitativ analysiert werden. Dieser Vergleich ist allerdings nur aussagekräftig, wenn das UV-Signal korrigiert wird. Das Signal des UV-Detektors wird hierzu mit dem Molekulargewicht multipliziert. Weiterhin wird die Mikrostruktur der Copolymere untersucht und mittels NMR-Analyse quantifiziert. Für die Messung der mittleren Molekulargewichte mit der GPC wird die Eichung mit Standards für Polybutadien verwendet.

\subsection{Einführung in die Materie}

1,3-Butadien enthaltende Copolymere zeichnen sich durch hervorragende Materialeigenschaften aus. So ist z.B. der Styrol-Butadien-Kautschuk (SBR) in seinen Eigenschaften eine gute Alternative zu Naturkautschuk und wird in vielfältigster Form verwendet. Aus diesem Grund ist es sinnvoll die RAFT-Polymerisation von 1,3-Butadien mit anderen Monomeren erstmalig systematisch zu untersuchen. Das Hauptaugenmerk liegt hierbei auf einer erfolgreichen Polymerisationskontrolle der RAFT-Agenzien. Die kinetischen Grundlagen der statistischen radikalischen Polymerisation sind bereits in den theoretischen Grundlagen (Kapitel 3.3) erläutert worden und müssen an dieser Stelle nicht wiederholt werden. Als Monomere für die Copolymerisation mit 1,3-Butadien (BD) werden n-Butylacrylat (BA) und Isopren (IP) verwendet. Das Ziel der Untersuchungen ist die Herstellung von Copolymeren mit verschiedenen Monomerkombinationen aus der Kautschukindustrie mit Hilfe der RAFT-Polymerisation. Aufgrund der hervorragenden Materialeigenschaften von Polybutadien und den entsprechenden Copolymeren erschließt sich ein breites Forschungsspektrum ${ }^{[51-57]}$. Auch die RAFT-Polymerisation ${ }^{[58-61]}$ mit 
anderen Comonomeren ist bereits erfolgreich durchgeführt worden. Als weitere lebende Polymerisationsform hat sich die $\mathrm{ATRP}^{[62-65]}$ als zuverlässige Variante etabliert, um gezielte Polymerarchitekturen $\mathrm{zu}$ generieren. Aufgrund der Erfahrungen in der Polymerisation von 1,3-Butadien ${ }^{[39]}$ wurde n-Butylacrylat als weiteres Comonomer verwendet, um höhere Molekulargewichte bei identischen Polymerisationszeiten zu erhalten.

\subsection{Statistische Copolymerisation von 1,3-Butadien (BD) und Butylacrylat (BA)}

Bei den Lösungsmittelpolymerisationen von 1,3-Butadien und Butylacrylat werden die Stoffmengenverhältnisse der beiden Monomeren von 1:1 immer konstant gehalten. Die Polymerisationstemperatur liegt bei $T=87^{\circ} \mathrm{C}$ und die Reaktionsdauer ist einheitlich $t=24 \mathrm{~h}$.

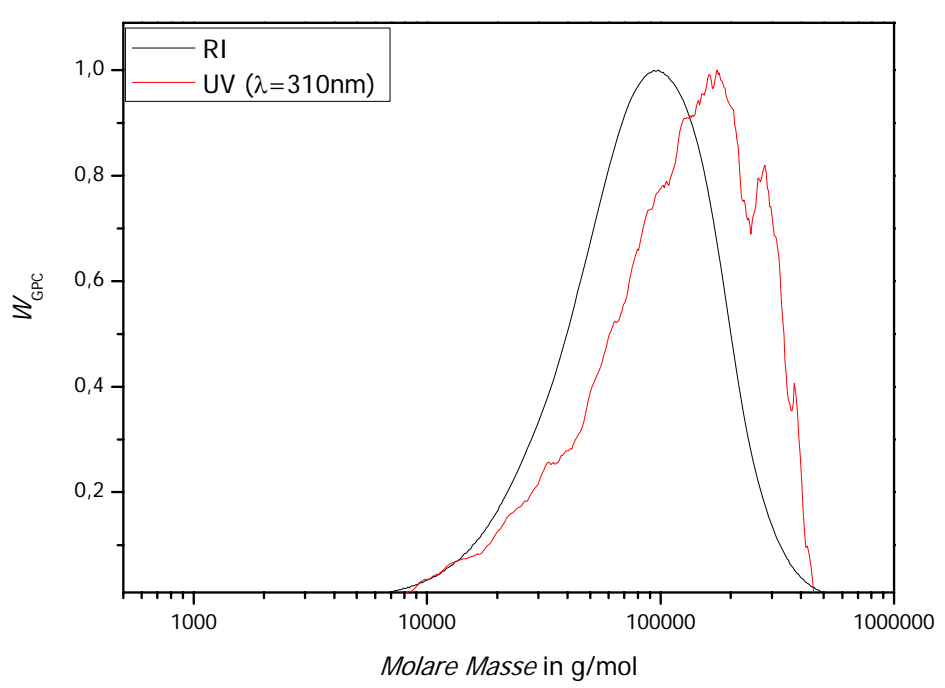

\section{Abbildung 7.2.1}

Auftragung der normierten GPC-Detektorsignale gegen die Molmasse. Zusammensetzung: $c_{0}($ Toluol $)=26 \mathrm{Mol} \%, c_{0}(C D B)=c_{0}(A C C)=0,005 \mathrm{Mol} \% . c_{0}(B D)=c_{0}(B A)=37 \mathrm{Mol} \%$, Auswertung: $U=6,62 \% ; M_{\mathrm{n}}=60741 \mathrm{~g} / \mathrm{mol}, M_{\mathrm{n}}^{\text {theo }}=54509 \mathrm{~g} / \mathrm{mol}, P D I=1,66$. 


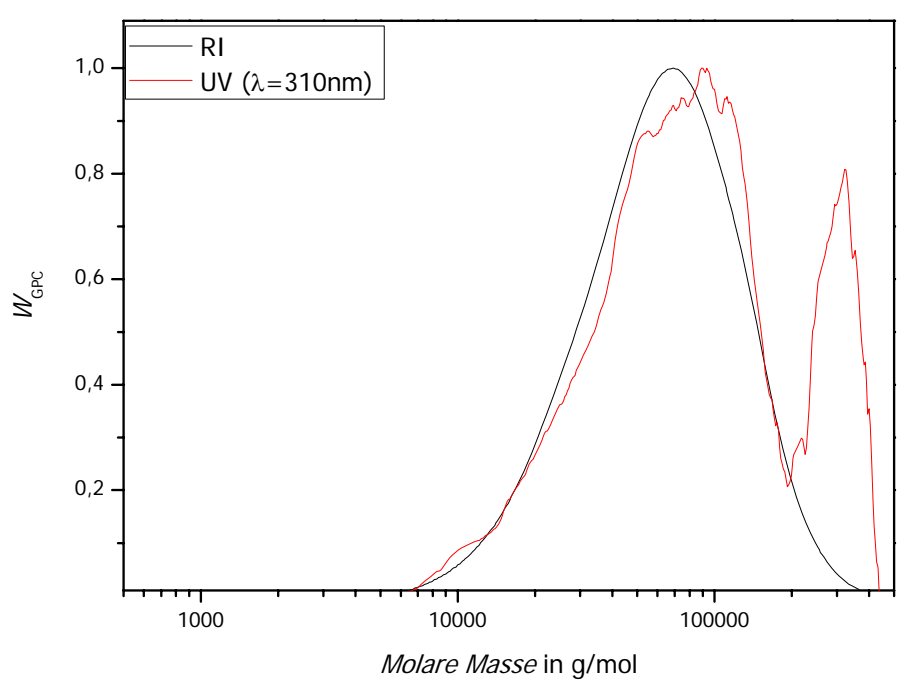

Abbildung 7.2.2

Auftragung der normierten GPC-Detektorsignale gegen die Molmasse. Zusammensetzung: $c_{0}($ Toluol $)=26 \mathrm{Mol} \%, c_{0}(C D B)=c_{0}(A C C)=0,010 \mathrm{Mol} \%, c_{0}(B D)=c_{0}(B A)=37 \mathrm{Mol} \%$, Auswertung: $U=9,20 \%, M_{\mathrm{n}}=46667 \mathrm{~g} / \mathrm{mol}, M_{\mathrm{n}}^{\text {theo }}=36927 \mathrm{~g} / \mathrm{mol}, P D I=1,62$.

In Abbildung 7.2.2 ist die Konzentration des RAFT-Agens und die Initiatorkonzentration bei der RAFT-Polymerisation im Vergleich zu Abbildung 7.2.1 verdoppelt worden. Es ist zu erwarten, dass kleinere Molekulargewichte bei höheren Umsätzen erreicht werden. Auch in diesem Fall ist eine deutliche Absorbanz des UV-Signals im makromolekularen Bereich der Molekulargewichtsverteilung sichtbar. Allerdings ist auf der hochmolekularen Seite eine deutliche Absorbanz des UV-Signals zu sehen. Dieses Ergebnis kann nicht reproduziert werden und kann deshalb als Artefakt betrachtet werden. Das Signal-Rausch-Verhältnis hat sich ebenfalls verbessert, da sich aufgrund der höheren Konzentration des RAFT-Agens das Verhältnis von Kettenlänge zu RAFT-Gruppe verkleinert hat. 


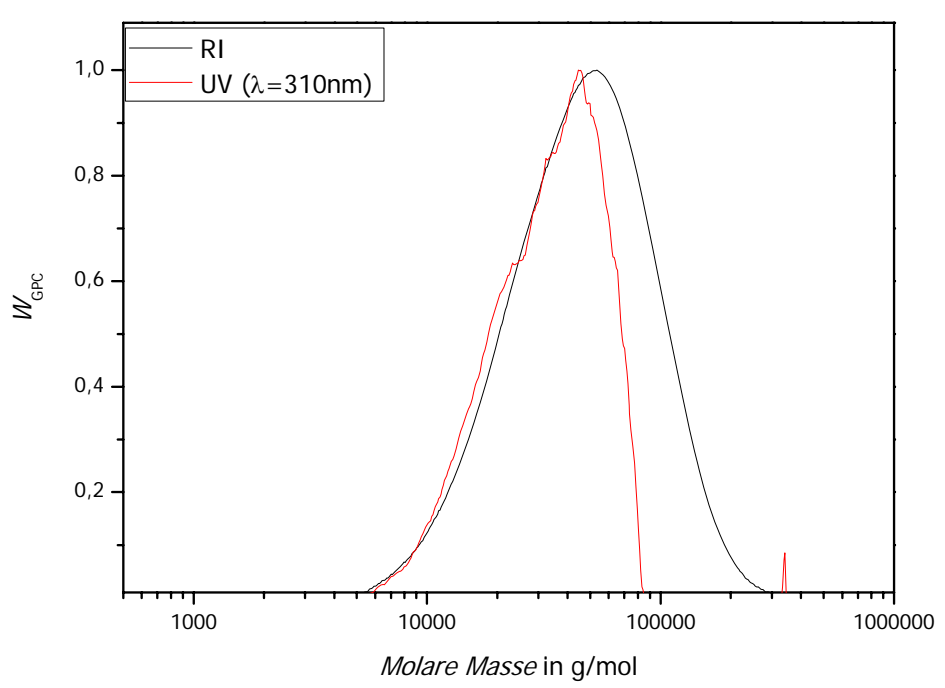

\section{Abbildung 7.2.3}

Auftragung der normierten GPC-Detektorsignale gegen die Molmasse. Zusammensetzung: $c_{0}($ Toluol $)=26 \mathrm{Mol} \%, c_{0}(C D B)=c_{0}(A C C)=0,020 \mathrm{Mol} \%, c_{0}(B D)=c_{0}(B A)=37 \mathrm{Mol} \%$, Auswertung: $U=14,93 \%, M_{\mathrm{n}}=35059 \mathrm{~g} / \mathrm{mol}, M_{\mathrm{n}}^{\text {theo }}=31677 \mathrm{~g} / \mathrm{mol}, P D I=1,61$.

Auch in der Abbildung 7.2.3 werden gute Reaktionskontrollen des RAFT-Agens Cumyldithiobenzoat (CDB) erzielt. Hier befinden sich die UV-Signale ebenfalls im makromolekularen Bereich. Eine deutliche Verbesserung der Reaktionskontrolle mit zunehmender Konzentration an RAFT-Agens kann nicht festgestellt werden, auch wenn sich die Polydispersität leicht verkleinert. Wie zu erwarten, kommt es zu einer Verringerung der mittleren Molekulargewichte, wenn die Konzentration an RAFT-Agens erhöht wird. Die experimentellen Umsätze passen auch gut mit den in der Theorie erwarteten Werten überein. Die Copolymerzusammensetzungen sind in Tabelle 7.2.1 angegeben, welche identisch sind mit den Ergebnissen der konventionellen radikalischen Polymerisation (vgl. Abbildung 3.3.3). Dieser Umstand lässt sich bei Betrachtung des Wachstumsschrittes erklären. Die Anlagerung von Monomereinheiten erfolgt nur an die aktive Spezies und ist somit nicht vom RAFT-Mechanismus abhängig. Aus diesem Grund ist die Kinetik der statistischen Copolymerisation unverändert. 


\section{Statistische Copolymerisation}

Tabelle 7.2.1

Auflistung der Zusammensetzung des Polymers der statistischen Copolymerisation von 1,3Butadien (BD) und Butylacrylat (BA) mit CDB als RAFT-Agens: Die Bezeichnungen PBD (Polybutadien) und PBA (Polybutylacrylat) beschreiben die Anteile der jeweiligen Monomere im Copolymer, welche mit Hilfe der NMR-Spektroskopie[66] bestimmt wurden. In der letzten Spalte wird die Glasübergangstemperatur des Polymers angegeben:

\begin{tabular}{|c|c|c|c|}
\hline Abbildung & $P B D$ in $\mathrm{Mol} \%$ & $P B A$ in $\mathrm{Mol} \%$ & $T_{\mathrm{G}}$ in ${ }^{\circ} \mathrm{C}$ \\
\hline 7.2 .1 & 65,5 & 34,5 & -79 \\
\hline 7.2 .2 & 66,6 & 33,4 & -77 \\
\hline 7.2 .3 & 66,4 & 33,6 & -84 \\
\hline
\end{tabular}

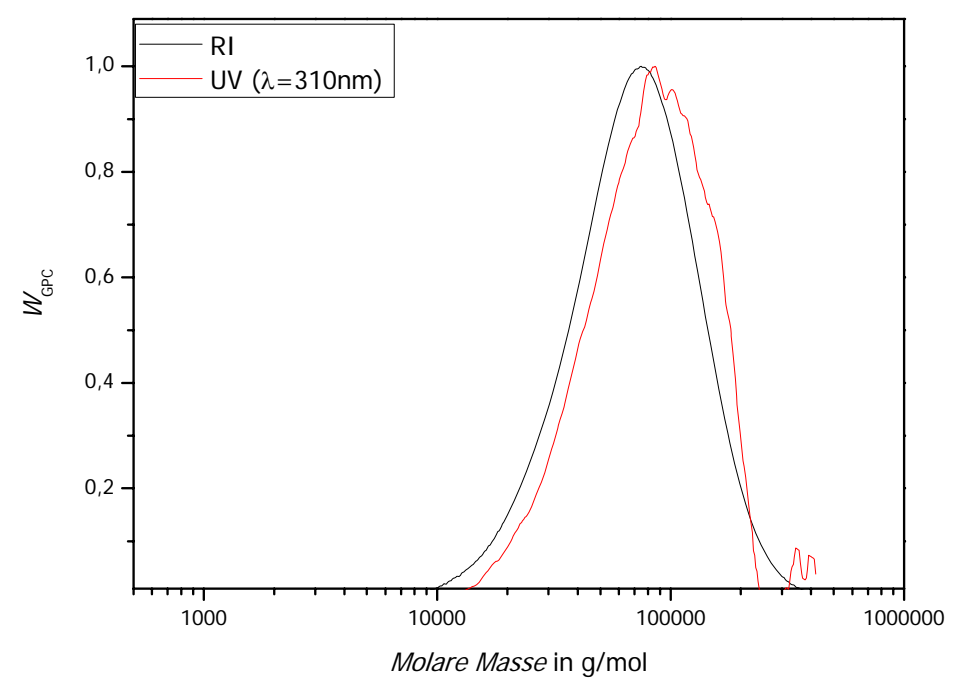

\section{Abbildung 7.2.4}

Auftragung der normierten GPC-Detektorsignale gegen die Molmasse. Zusammensetzung: $c_{0}($ Toluol $)=26 \mathrm{Mol} \%, \quad c_{0}(B P E T C)=c_{0}(A C C)=0,005 \mathrm{Mol} \%, \quad c_{0}(B D)=c_{0}(B A)=37 \mathrm{Mol} \%$, Auswertung: $U=5,72 \%, M_{\mathrm{n}}=56487 \mathrm{~g} / \mathrm{mol}, M_{\mathrm{n}}^{\text {theo }}=47172 \mathrm{~g} / \mathrm{mol}, P D I=1,45$.

Die Ergebnisse der Lösungsmittelpolymerisationen von 1,3-Butadien und Butylacrylat unter Verwendung von Bis(1-phenylethyl)trithiocarbonat (BPETC) als RAFT-Agens werden in den Abbildungen 7.2.4, 7.2.5 und 7.2.6 aufgezeigt. Bei allen drei Darstellungen ist ein Signal des UV-Detektors im makromolekularen Bereich zu erkennen, welches auf eine erfolgreiche Reaktionskontrolle schließen lässt. Das Signal-Rausch-Verhältnis des UV-Signals ist für die RAFT-Polymerisationen mit BPETC besser. Es ist anzunehmen, 
dass die Absorbanz des Trithiocarbonats in diesem Fall bei der betrachteten Wellenlänge von $\lambda=310 \mathrm{~nm}$ höher ist.

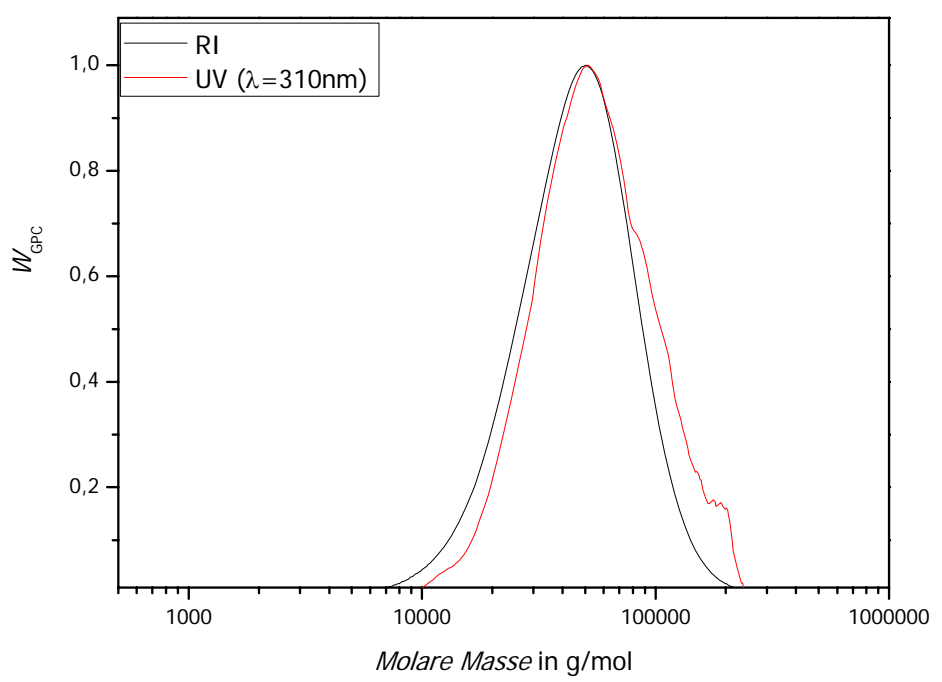

\section{Abbildung 7.2.5}

Auftragung der normierten GPC-Detektorsignale gegen die Molmasse. Zusammensetzung: $c_{0}($ Toluol $)=26 \mathrm{Mol} \%, \quad c_{0}($ BPETC $)=c_{0}(A C C)=0,010 \mathrm{Mol} \%, \quad c_{0}(B D)=c_{0}(B A)=37 \mathrm{Mol} \%$, Auswertung: $U=7,82 \%, M_{\mathrm{n}}=38258 \mathrm{~g} / \mathrm{mol}, M_{\mathrm{n}}^{\text {theo }}=33134 \mathrm{~g} / \mathrm{mol}, P D I=1,37$.

In diesen drei Experimenten wurde die Konzentration an RAFT-Agens und Initiator schrittweise verdoppelt. Auch in diesem Fall kann eine gute Übereinstimmung zwischen experimentellen und theoretischen Umsätzen erzielt werden. Auffällig ist, dass die theoretischen Molekulargewichte $M_{\mathrm{n}}^{\text {theo }}$ im Vergleich zu den experimentellen Werten alle deutlich zu klein ausfallen. Die Erklärung ist in der Berechnung der theoretischen Werte und in der Eichung der GPC zu suchen. Die Eichung der GPC erfolgte mit PolybutadienStandards und ist somit nur für ein Polybutadien repräsentativ. Für die Berechnung nach Gleichung 3.6.1 wird für die Molmasse des Monomers der Wert für 1,3-Butadien $(M=54,09 \mathrm{~g} / \mathrm{mol})$ angenommen. Allerdings ist $\mathrm{zu}$ beachten, dass die Molmasse von Butylacrylat mit $M=128,17 \mathrm{~g} / \mathrm{mol} \mathrm{mehr}$ als zweimal so groß ist. Die theoretischen Molekulargewichte würden dementsprechend auch größer ausfallen. Die experimentellen Werte liegen also zwischen den beiden Grenzfällen der möglichen theoretischen Molekulargewichte. Generell ist es schwierig bei statistischen Copolymeren Aussagen über absolute Werte von Molekulargewichten zu treffen, welche über die GPC bestimmt 


\section{Statistische Copolymerisation}

worden sind. Allerdings können diese Werte einen Anhaltspunkt liefern und als Orientierung dienen.

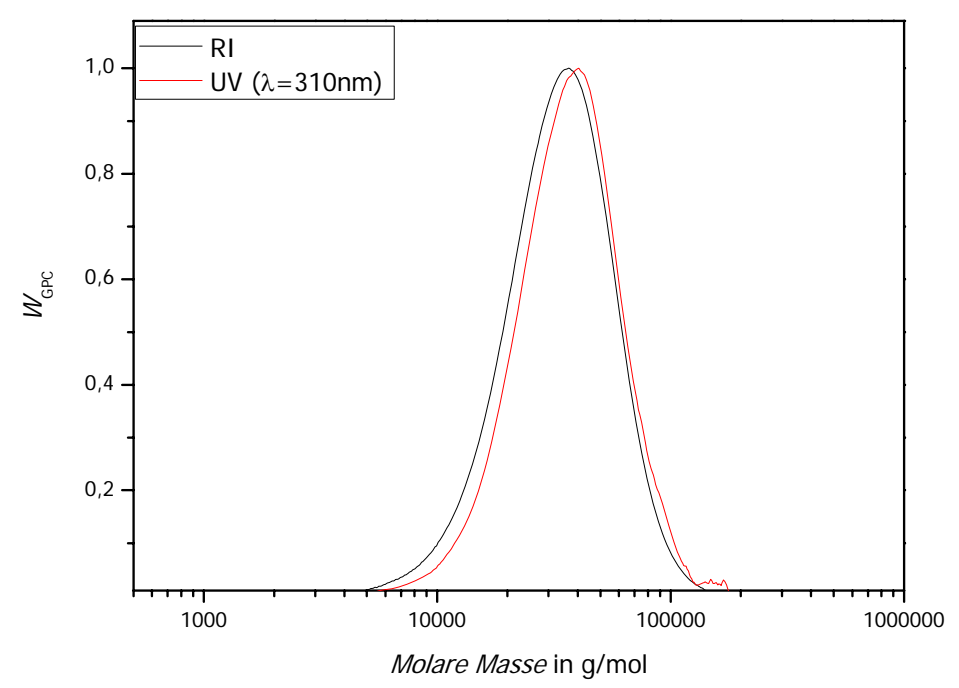

\section{Abbildung 7.2.6}

Auftragung der normierten GPC-Detektorsignale gegen die Molmasse. Zusammensetzung: $c_{0}($ Toluol $)=26 \mathrm{Mol} \%, \quad c_{0}($ BPETC $)=c_{0}(A C C)=0,020 \mathrm{Mol} \%, \quad c_{0}(B D)=c_{0}(B A)=37 \mathrm{Mol} \%$, Auswertung: $U=12,75 \%, M_{\mathrm{n}}=27591 \mathrm{~g} / \mathrm{mol}, M_{\mathrm{n}}^{\text {theo }}=27881 \mathrm{~g} / \mathrm{mol}, P D I=1,34$.

In Tabelle 7.2.2 sind die Anteile an Polybutadien und Polybutylacrylat des Copolymers der RAFT-Polymerisation mit Bis(1-phenylethyl)trithiocarbonat (BPETC) angegeben. In der theoretischen Betrachtung sollte der Wechsel des RAFT-Agens keinen Einfluss auf die Copolymerzusammensetzung haben, da die Propagation in Lösung unabhängig vom RAFT-Agens abläuft. Im Vergleich zu den Ergebnissen der RAFT-Polymerisation mit Cumyldithiobenzoat sind keine signifikanten Unterschiede zu erkennen. Auch in diesem Fall werden die Voraussagen des Copolymerisationsdiagramms sehr gut wiedergegeben (vgl. Abbildung 3.3.3). 
Tabelle 7.2.2

Auflistung der Zusammensetzung des Polymers der statistischen Copolymerisation von 1,3Butadien (BD) und Butylacrylat (BA) mit BPETC als RAFT-Agens: Die Bezeichnungen PBD (Polybutadien) und PBA (Polybutylacrylat) beschreiben die Anteile der jeweiligen Monomere im Copolymer, welche mit Hilfe der NMR-Spektroskopie[66] bestimmt wurden. In der letzten Spalte wird die Glasübergangstemperatur des Polymers angegeben:

\begin{tabular}{|c|c|c|c|}
\hline Abbildung & $P B D$ in $\mathrm{Mol} \%$ & $P B A$ in $\mathrm{Mol} \%$ & $T_{\mathrm{G}}$ in ${ }^{\circ} \mathrm{C}$ \\
\hline 7.2 .4 & 67,7 & 32,3 & -79 \\
\hline 7.2 .5 & 66,2 & 33,8 & -85 \\
\hline 7.2 .6 & 63,6 & 36,4 & -85 \\
\hline
\end{tabular}

\subsection{Statistische Copolymerisation von 1,3-Butadien (BD) und Isopren (IP)}

In diesem Kapitel wird die statistische Copolymerisation von 1,3-Butadien und Isopren mit verschiedenen RAFT-Agenzien erstmalig qualitativ untersucht. Die strukturellen Ähnlichkeiten spiegeln sich auch im Polymerisationsverhalten der beiden Monomeren wider. Isopren bildet aufgrund der Methylgruppe etwas stabilere Radikale aus. Im großtechnischen Maßstab kann sich das synthetische Polyisopren nicht gegen die natürliche Variante durchsetzten. Der Hauptgrund dafür ist in der Mikrostruktur zu suchen. Der hohe Anteil an cis-1,4-Polyisopren von Naturkautschuk kann synthetisch nicht realisiert werden. Auch bei diesen Lösungsmittelpolymerisationen ist das Verhältnis von 1,3-Butadien und Isopren 1:1. Die Polymerisationstemperatur beträgt $T=87^{\circ} \mathrm{C}$ und die Polymerisationsdauer liegt bei $t=24 \mathrm{~h}$. Die Umsatzbestimmung erfolgte gravimetrisch. Eine gute Polymerisationskontrolle der beiden RAFT-Agenzien und die erfolgreiche Copolymerisation der beiden Monomere stehen im Vordergrund der Untersuchungen. Das Isopren wurde vor der Polymerisation frisch destilliert.

In Abbildung 7.3.1 ist die Molekulargewichtsverteilung der RAFT-Polymerisation mit CDB dargestellt. Das RAFT-Agens zeigt eine gute Polymerisationskontrolle, da die Absorbanz des UV-Signals im makromolekularen Bereich liegt. 


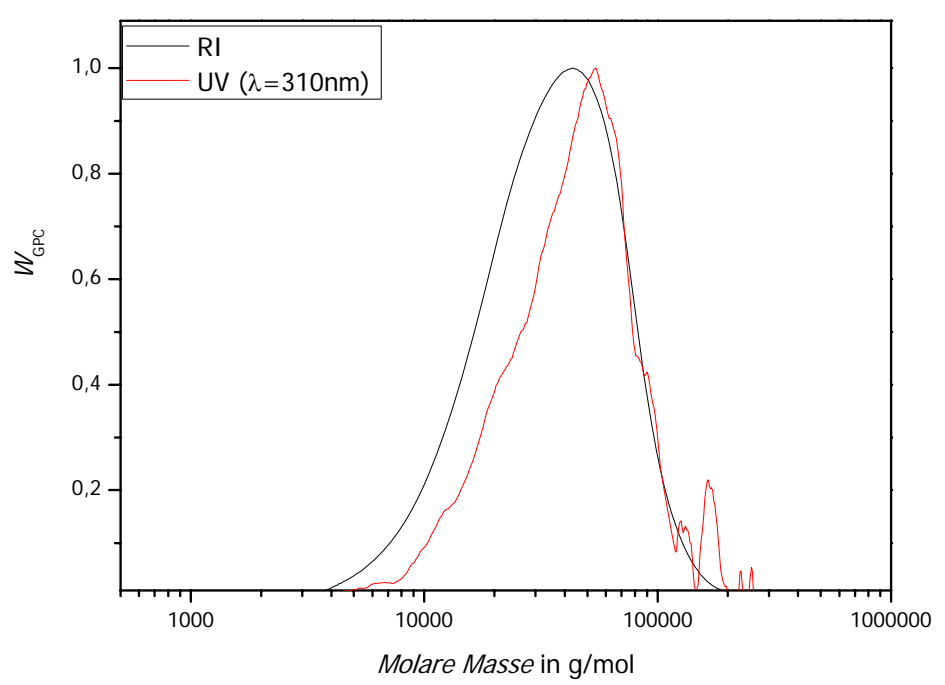

\section{Abbildung 7.3.1}

Auftragung der normierten GPC-Detektorsignale gegen die Molmasse. Zusammensetzung: $c_{0}($ Toluol $)=26 \mathrm{Mol} \%, c_{0}(C D B)=c_{0}(A C C)=0,010 \mathrm{Mol} \%, c_{0}(B D)=c_{0}(I P)=37 \mathrm{Mol} \%$, Auswertung: $U=5,92 \%, M_{\mathrm{n}}=24857 \mathrm{~g} / \mathrm{mol}, M_{\mathrm{n}}^{\text {theo }}=22186 \mathrm{~g} / \mathrm{mol}, P D I=1,57$.

Die Überlappung der beiden Kurven impliziert, dass jede Polymerkette auch in diesem Fall eine RAFT-Gruppe trägt. Die schlechte Deckung der beiden Signale auf der niedermolekularen Seite zeigt auf, dass auch „totes“ Polymer entstanden ist. Es kommt zu einem Hybridverhalten. In diesem Fall wird das Vorgleichgewicht der RAFTPolymerisation im Vergleich zur Gesamtpolymerisationszeit nicht schnell genug durchlaufen, wodurch in der Anfangsphase die Kinetik der konventionellen radikalischen Polymerisation überwiegt. Erst bei höheren Molekulargewichten kommt es zu einer guten Übereinstimmung zwischen der Absorbanz des UV-Signals und des Signals des Massendetektors. Dieses Verhalten zeigt sich auch in dem recht hohen Wert der Polydispersität von $P D I=1,57$. Das theoretische Molekulargewicht $M_{\mathrm{n}}^{\text {theo }}$ ist kleiner als die experimentell ermittelten Werte. Auch in diesem Fall wurde für die Berechnung die Molmasse des Monomers 1,3-Butadien verwendet. Die theoretischen Werte unter der Annahme, dass ein Homopolymer aus Isopren vorliegt, sind entsprechend größer und passen gut zu den experimentellen Werten. Auch hier spielt die Eichung der GPC eine wichtige Rolle in den Unterschieden zwischen den experimentellen und den theoretischen Molekulargewichten. 


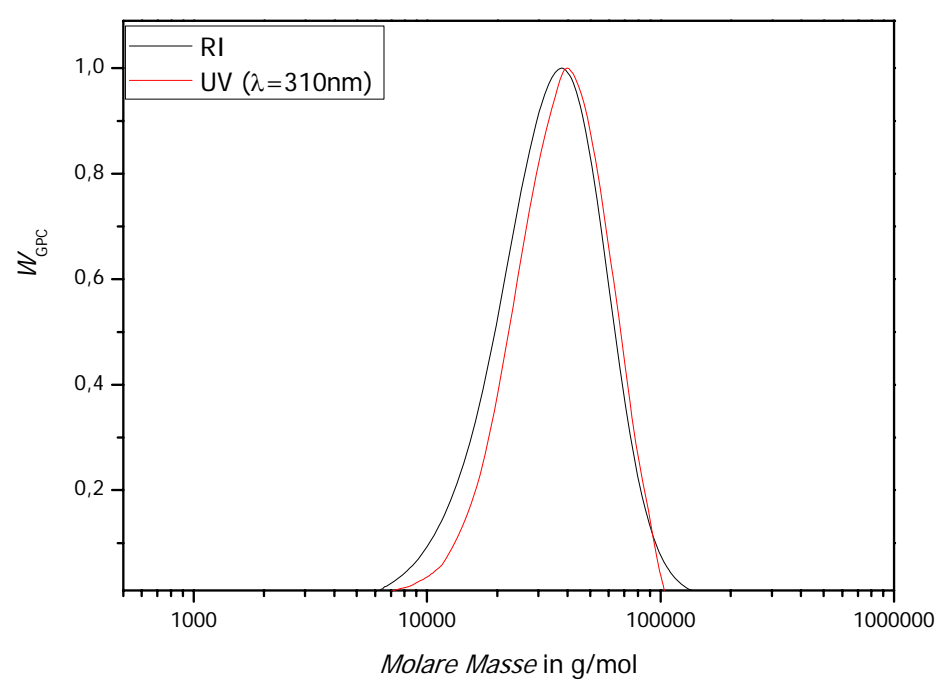

\section{Abbildung 7.3.2}

Auftragung der normierten GPC-Detektorsignale gegen die Molmasse. Zusammensetzung: $c_{0}($ Toluol $)=26 \mathrm{Mol} \%, \quad c_{0}($ BPETC $)=c_{0}(A C C)=0,010 \mathrm{Mol} \%, \quad c_{0}(B D)=c_{0}(B A)=37 \mathrm{Mol} \%$, Auswertung: $U=9,75 \%, M n=27236 \mathrm{~g} / \mathrm{mol}, M_{\mathrm{n}}^{\text {theo }}=34508 \mathrm{~g} / \mathrm{mol}, P D I=1,30$.

Die Molekulargewichtsverteilung in Abbildung 7.3.2 zeigt eine gute Polymerisationskontrolle der statistischen Copolymerisation von 1,3-Butadien und Isopren mit BPETC als RAFT-Agens. Die Absorbanz der RAFT-Gruppen liegt im makromolekularen Bereich und die Überlappung der beiden Verteilung ist sehr gut. Die Molekulargewichtsverteilung des Massensignals ist sehr schmal und indiziert mit einer Polydispersität von $P D I=1,30$ ebenfalls eine sehr gute Kontrolle der Polymerisation. Anscheinend ist das Trithiocarbonat mit den sekundären Abgangsgruppen für die RAFTPolymerisation von 1,3-Butadien und Isopren besser geeignet als das Cumyldithiobenzoat. 


\section{RAFT-Polymerisationen an Industrierußen}

In diesem Kapitel geht es um die RAFT-Polymerisation in der Gegenwart und an der Oberfläche von Rußen. Aus diesem Grund wird im ersten Abschnitt eine Einleitung in die Materie der Industrieruße gegeben. Es folgt eine Einführung in die Herstellungsverfahren, die Morphologie und die physikalischen bzw. chemischen Eigenschaften. Im weiteren Verlauf werden dann die bereits bekannten Möglichkeiten der Immobilisierung von funktionellen Gruppen und RAFT-Agenzien auf der Oberfläche erläutert und mit dem eigenen Verankerungsmechanismus evaluiert. Im Anschluss erfolgt die Auswertung der ersten Verankerungsversuche von Vorstufen der jeweiligen RAFT-Agenzien. Neben der quantitativen Analyse der Immobilisierung werden auch konventionelle radikalische Polymerisationen in Gegenwart von Rußpartikeln untersucht, um den Einfluss auf das Polymerisationsverhalten zu klären. In diesem Fall wird das Monomer 1,3-Butadien durch Styrol ersetzt. Aufgrund der leichteren Handhabbarkeit, der geringeren Toxizität und dem ähnlichen Polymerisationsverhalten ${ }^{[67,68]}$ eignet sich Styrol hervorragend als Substitut, um größere Probenmengen zu generieren. Im letzten Kapitel dieses Abschnittes werden dann die RAFT-Polymerisationen von der Oberfläche beschrieben und analytisch ausgewertet.

\subsection{Einführung in die Materie}

Ruß ist nicht gleich Ruß. Im deutschen Sprachgebrauch bezeichnet das Wort „Ruß“ den gezielt hergestellten Industrieruß (engl. carbon black) und gleichzeitig die unerwünschten Nebenprodukte in den Abgasen von Verbrennungsprozessen (engl. soot).

Der Industrieruß wird unter definierten Bedingungen hergestellt und ist in seinen physikalischen und chemischen Eigenschaften eindeutig bestimmt. Bereits vor mehr als 20000 Jahren wurde Ruß als Schwarzpigment in der Höhlenmalerei verwendet. Die geschichtliche Verwendung von Ruß beschränkte sich für eine sehr lange Zeit auf die Verwendung als Druckerschwärze oder Tintenruß. Meistens wurde der Ruß durch unvollständige Verbrennung von Pflanzenölen, Kienöl (Terpentin) und Holzteer hergestellt. Die Abscheidung erfolgte an kalten oder gekühlten Gegenständen, an denen der entstandene Ruß abgeschabt werden konnte. 


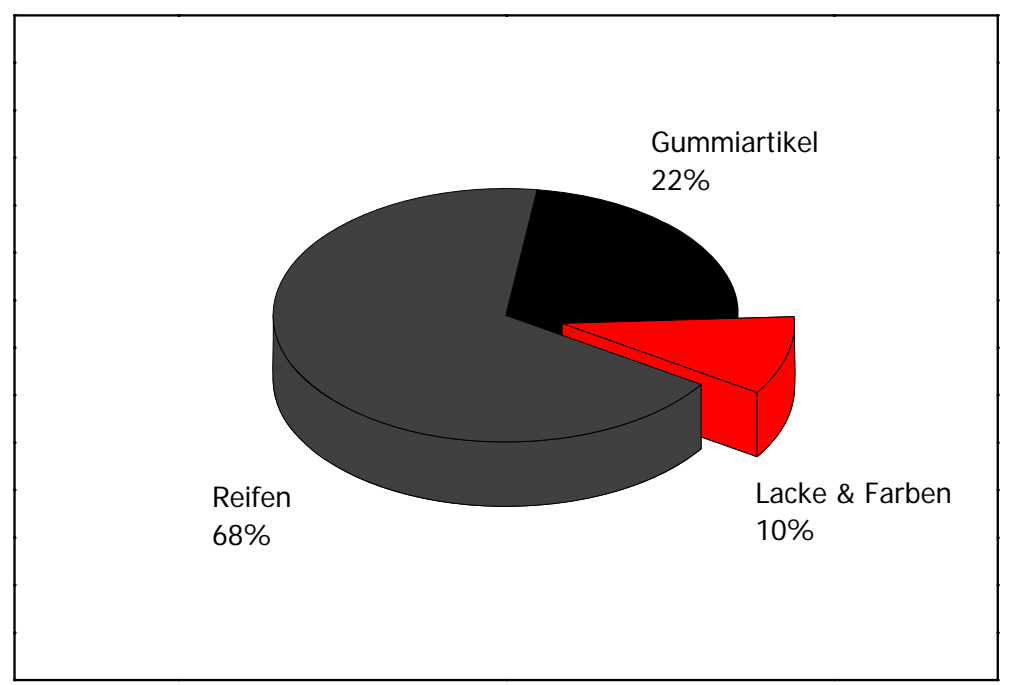

\section{Abbildung 8.1.1}

Darstellung der Absatzstruktur von Industrierußen (Stand 1998) in der Produktion[69].

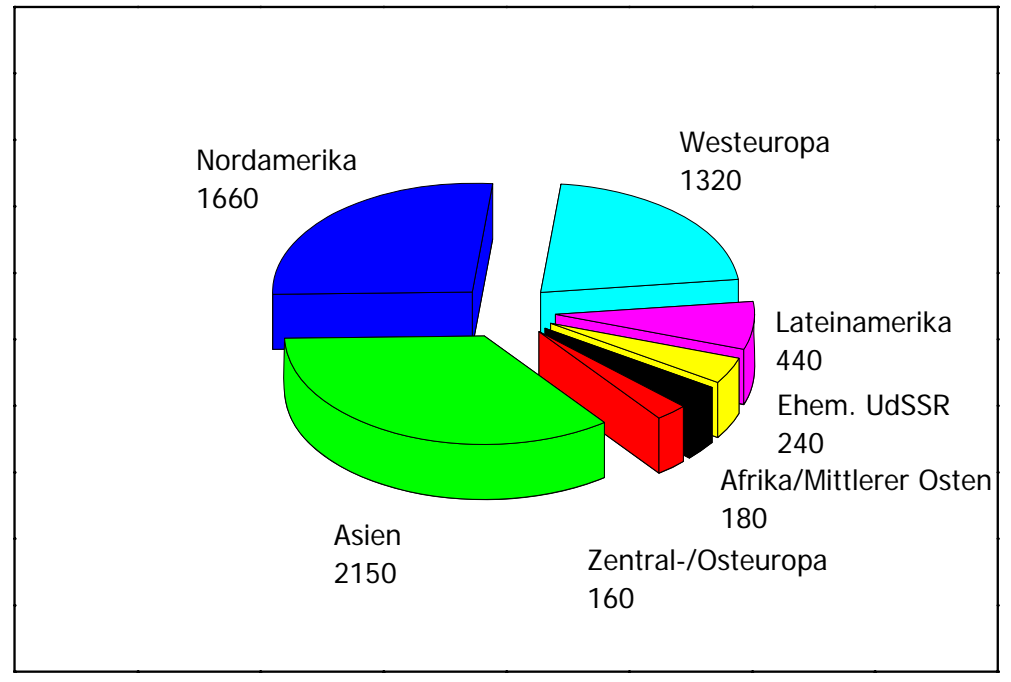

\section{Abbildung 8.1.2}

Darstellung der regionalen Aufteilung der Produktionskapazität von Industrierußen in 1000 Tonnen.

Im 19. Jahrhundert entwickelte sich Steinkohleteer zu einem weiteren wichtigen Rohstoff in der Herstellung von Ruß. Im Jahre 1822 wurde der Channel Black Prozess in den USA 


\section{RAFT-Polymerisation an Industrierußen}

entwickelt, bei dem Erdgas kontinuierlich einer unvollständigen Verbrennung zugeführt wurde. Dieser Prozess gewann großtechnische Bedeutung, als die verstärkenden Eigenschaften des Rußes in vulkanisierten Kautschuken entdeckt wurden.

Durch die Verwendung von Ruß als Füllmaterial in Autoreifen kann die Laufleistung um ein Vielfaches verlängert werden. Demnach ist es auch nicht verwunderlich, dass heutzutage ungefähr $68 \%$ des hergestellten Rußes in der Reifenherstellung verwendet werden (siehe Abbildung 8.1.1).

In den 30er Jahren des letzten Jahrhunderts wurde das „Deutsche Gasrußverfahren“ von Degussa $^{\circledR}$ entwickelt, wobei als Rohstoffe Steinkohleteer-Aromaten Anwendung fanden. Das noch heute dominierende Herstellungsverfahren wurde bereits in den 1920er Jahren entwickelt und ist als Furnace Black Prozess ${ }^{[70]}$ bekannt.

\subsection{Physikalische Eigenschaften}

\subsubsection{Morphologie}

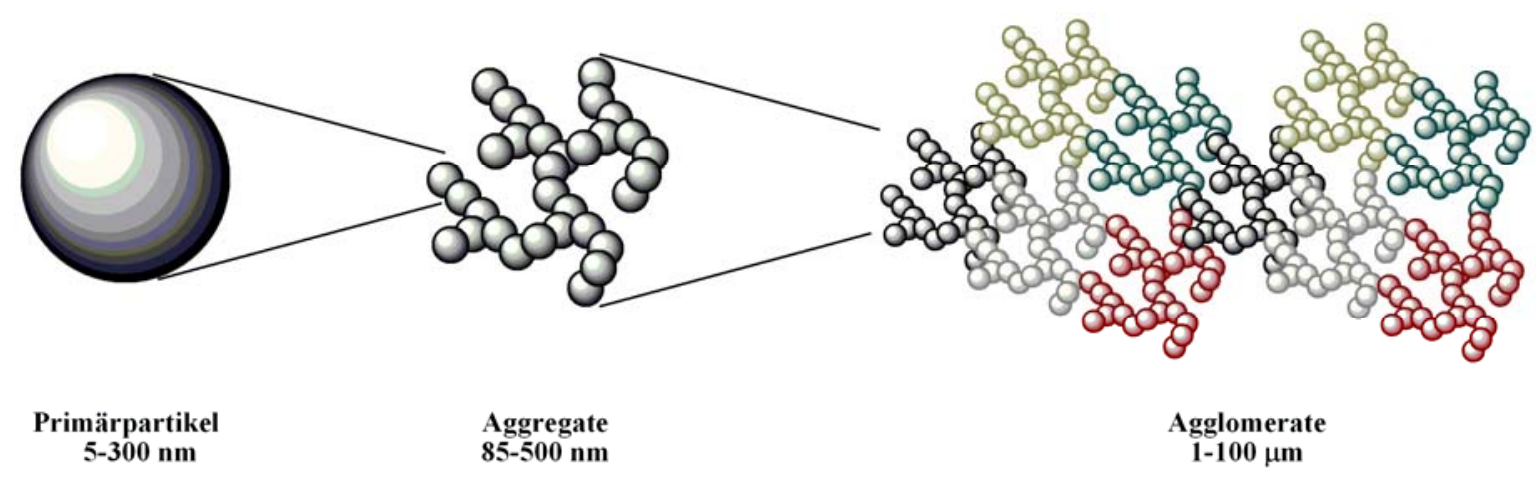

Abbildung 8.2.1

Schematische Darstellung der unterschiedlichen Morphologien von Industrierußen mit Angabe der Größenverhältnisse.

In der Beschreibung der Morphologie von Industrierußen müssen drei Begriffe unterschieden und definiert werden.

- Primärpartikel: Diese meistens sphärischen Partikel stellen die kleinste Struktureinheit dar und kondensieren in Aggregaten zusammen.

- Aggregate: Aggregate sind die kleinste dispergierbare Einheit der Industrieruße. 
- Agglomerate: Agglomerate entstehen aus Zusammenlagerungen von Aggregaten, welche durch Van-der-Waals-Kräfte oder durch Bindemittel beieinander gehalten werden.

Die Grundstruktur der Primärpartikel entsteht aus einer Polykondensation und Dehydrierung von Kohlenwasserstoffen zu aromatischen Systemen. Diese ähneln in der Struktur sehr stark dem Graphit und bilden den Kondensationskeim für die Anlagerung von weiteren Kohlenstoffschichten. Die Ausrichtung dieser Schichten erfolgt dabei parallel zu den bereits vorhandenen Oberflächen.

Dadurch kommt es zur Ausbildung einer Kugelschalentextur, welche auch gerne als Zwiebelmodell bezeichnet wird. Die Größe der Primärpartikel kann in Abhängigkeit der Prozessführung und des Herstellungsverfahrens zwischen $5 \mathrm{~nm}$ und $300 \mathrm{~nm}$ variieren.

Die makroskopischen Eigenschaften des Industrierußes, wie die Dispergierbarkeit, Farbtiefe und Adsorptionsvermögen hängen sehr stark von der Primärpartikelgröße ab. So ergibt sich bei einem kleineren Partikeldurchmesser eine größere spezifische Oberfläche, welche im Umkehrschluss einen direkten Einfluss auf die Adsorption hat. Demnach ist bei kleineren Partikeln auch die Tendenz der Zusammenlagerung in Aggregate stärker ausgeprägt, da mehr Kontaktfläche zur Verfügung steht. Die Form der Aggregate kann wiederum durch Prozessparameter in der Herstellung variiert werden.

Es kommt zu einer Unterscheidung zwischen sphärischen, elliptischen, linearen und verzweigten Aggregaten (Abbildung 8.2.2). Diese Aggregate bilden die kleinste dispergierbare Einheit der Industrieruße und können nur durch sehr starke mechanische Einwirkung verändert werden. 
Sphärisch

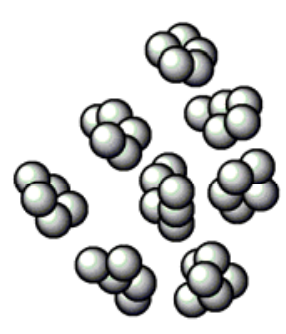

Verzweigt

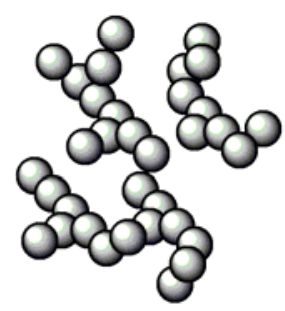

Elliptisch

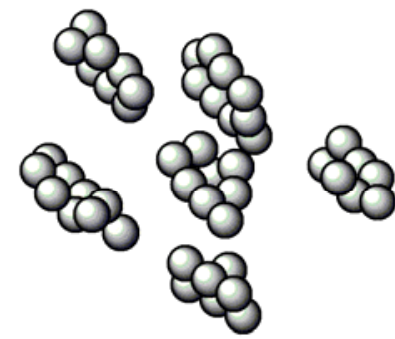

Linear

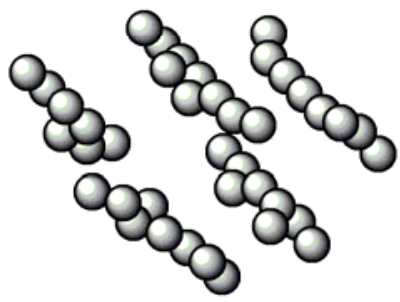

Abbildung 8.2.2

Darstellung der verschiedenen Grundstrukturen in der Aggregatentstehung.

In den Aggregaten können mittels Röntgenbeugung verschiedene kristalline Bereiche nachgewiesen werden. Es handelt sich dabei nicht um Schichtabschnitte, sondern um Bezirke, bei denen die Kohlenstoffschichten parallel und im gleichen Abstand zueinander angeordnet sind. Die Aggregate lagern sich $\mathrm{zu}$ Agglomerate zusammen. Diese Eigenschaft wird bei der Ausscheidung des Rußes aus den Reaktionsgasen im Herstellungsprozess genutzt. Die Agglomerate sind recht locker gebunden und lassen sich durch mechanische Krafteinwirkung leicht in ihrer Form verändern. Im Vergleich zum Graphit mit einem Schichtabstand von 0,335 nm variieren die Abstände der einzelnen Schichten des Industrierußes zwischen 0,35-0,4 nm. Aus diesem Grund fällt auch die Dichte kleiner aus und kann Werte von $1,8 \mathrm{~g} / \mathrm{cm}^{3}$ bis $2,1 \mathrm{~g} / \mathrm{cm}^{3}$ annehmen (Graphit 2,255 g/cm ${ }^{3}$ ). Die Unterschiede in den physikalischen Eigenschaften lassen sich durch Schicht- und Gitterdefekte erklären, welche allerdings bei höheren Temperaturen ausgeheilt werden können. Die sogenannte Graphitierung setzt ab $1200{ }^{\circ} \mathrm{C}$ ein. Zwischen 2500-3000 ${ }^{\circ} \mathrm{C}$ erfolgt eine Umorientierung der Schichten des Rußes, wodurch kleine Graphitkristalle entstehen. Der Ruß erhält dadurch eine polyedrische Gestalt. Dieser Vorgang hat allerdings kaum einen Einfluss auf die Struktur des Rußes, da die Aggregation und die Partikelgröße unverändert bleiben. 
Die spezifische Oberfläche von Industrierußen hängt sehr stark von der Partikelgröße ab und kann dementsprechend in einem weiten Bereich variiert werden. Grobe Thermalruße haben eine spezifische Oberfläche von $10 \mathrm{~m}^{2} / \mathrm{g}$ während Pigmentruße diesen Wert um das Hundertfache übertreffen. In der Regel liegen die spezifischen Oberflächen für Industrieruße, welche in der Herstellung von Gummiartikeln verwendet werden, in der Größenordnung von $70-150 \mathrm{~m}^{2} / \mathrm{g}$.

Industrieruße besitzen eine gewisse elektrische Eigenleitfähigkeit, welche vom Übergangswiderstand zwischen den Partikeln bestimmt wird. Dieser Übergangswiderstand verändert sich mit der Konzentration und der Anordnung der Rußpartikel im Gesamtsystem. Die Leitfähigkeit unterbindet eine elektrostatische Aufladung von Polymeren und Kautschuken.

Die Lichtabsorption im sichtbaren Bereich ist sehr groß. Das Absorptionsvermögen nimmt mit abnehmender Partikelgröße zu und liegt teilweise bei 99,8 \% für Pigmentruße, welche zur Schwarzfärbung verwendet werden. Industrieruße werden auch zur UVStabilisierung von Kunststoffen eingesetzt, da eine Absorption auch im UV- und IRBereich erfolgt.

\subsection{Chemische Eigenschaften}

Die chemischen Eigenschaften von Industrierußen korrelieren mit der Konzentration von Heteroatomen im Kohlenstoffgerüst. Die chemische Zusammensetzung ist hierbei wieder ein direkter Spiegel des Herstellungsprozesses und der chemischen Nachbehandlung. Hier ist es entscheidend, ob der Ruß unter reduzierender oder unter oxidierender Atmosphäre hergestellt wurde. Die meisten Heteroatome sind an den Schichtgrenzen und den Defektstellen gebunden und können in verschiedensten funktionellen Gruppen vorliegen (Abbildung 8.3.1). Furnaceruße, welche in der Reifenproduktion eingesetzt werden, haben einen Kohlenstoffgehalt von über 99 \%. Als weitere Bestandteile sind Wasserstoff, Schwefel und Sauerstoff $\mathrm{zu}$ nennen, welche in unterschiedlichen Konzentrationen vorliegen. Die Oberflächenoxide zersetzen sich oberhalb von $300{ }^{\circ} \mathrm{C}$ unter Abspaltung von Wasser, Kohlenmonoxid und Kohlendioxid. Als übliche Methode zur Bestimmung des Oxidationsgrades dient die Bestimmung des Glühverlustes bei $950{ }^{\circ} \mathrm{C}$. In Tabelle 8.3.1 werden die Zusammensetzungen von Thermalrußen und Gummirußen wiedergegeben. 


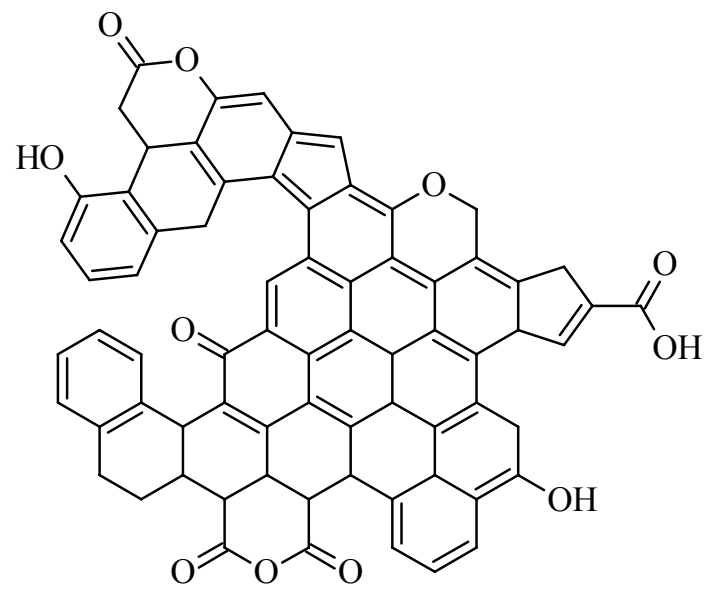

\section{Abbildung 8.3.1}

Beispielhafte Darstellung der Oberfläche von Industrierußen mit sauerstoffhaltigen funktionellen Gruppen: Die Heteroatome sitzen fast ausschließlich an den Rändern der Schichten und an Defektstellen.

Der Aschegehalt der Industrieruße beträgt nur wenige Zehntelprozent und resultiert aus Verunreinigungen des Rohstoffes, aus dem Prozesswasser der Herstellung und aus der Zugabe von Salzen für die Strukturoptimierung.

Tabelle 8.3.1

Zusammensetzung unterschiedlicher Rußtypen in Abhängigkeit des Herstellungsprozesses in Prozent [71]:

\begin{tabular}{ccccccc}
\hline Typ & Kohlenstoff & Wasserstoff & Sauerstoff & Schwefel & Asche & Flüchtig \\
\hline Furnaceruß & $97,3-99,3$ & $0,20-0,40$ & $0,20-1,20$ & $0,20-1,20$ & $0,10-1,00$ & $0,60-1,50$ \\
\hline Thermalruß & 99,4 & $0,30-0,50$ & $0,00-0,12$ & $0,00-0,25$ & $0,20-0,38$ & $<0,40$
\end{tabular}

Der Oxidationsgrad der Rußoberfläche kann des Weiteren noch durch eine oxidative Nachbehandlung nach oben korrigiert werden, wodurch ein Sauerstoffgehalt von bis zu $15 \%$ erreicht werden kann. Bei diesem Prozess entstehen in erster Linie saure Oberflächenoxide, so dass der Ruß in wässriger Suspension einen $\mathrm{pH}$-Wert kleiner als 7 aufweist. Aufgrund des erhöhten Sauerstoffgehaltes kommt es zu einer besseren Benetzung mit organischen Binde- und Lösungsmitteln. Technisch bedeutsam ist die Oxidation mit Salpetersäure ${ }^{[69]}$. 


\subsection{Herstellungsprozesse}

\subsubsection{Das Furnaceruß-Verfahren}

Durch die Leistungsfähigkeit, Umweltverträglichkeit und Flexibilität in der Variation der Prozessparameter dominiert das Furnaceruß-Verfahren die Herstellung von Industrierußen. Neben der spezifischen Oberfläche und der Rußstruktur können auch besondere Eigenschaften der Anwendung, wie der Bindemittelbedarf, die Farbtiefe und der Elastizitätsmodul von Gummiartikeln in einem weiten Bereich variiert und eingestellt werden.

Im Mittelpunkt des Furnaceruß-Verfahren steht der kontinuierliche Strömungsreaktor, der sogenannte Rußreaktor. Die Umsetzung der Rohstoffe zu den gewünschten Industrierußen erfolgt in einem geschlossenen System. Die Reaktorkammer lässt sich schematisch in vier Zonen unterteilen. Man unterscheidet hierbei die Verbrennungskammer, die Mischzone, die Reaktionszone und die Quenchzone.

Durch die Umsetzung von Erdgas oder Kokereigas werden in der Verbrennungskammer die benötigten Temperaturen für die Spaltung der Rohstoffe erhalten. Die Verbrennungsluft erreicht dabei eine Temperatur von 500-800 ${ }^{\circ} \mathrm{C}$. Es werden größere Luftmengen eingesetzt, als für die vollständige Umsetzung des Brennstoffs benötigt werden, da die für die Pyrolysereaktion benötigte Energiemenge auch durch partielle Verbrennung der Rußrohstoffe erzeugt wird. Größere Luftmengen führen zu höheren Temperaturen im Reaktionsraum und zu kleineren Ausbeuten, da ein Großteil des Rohstoffs ebenfalls verbrennt. Die heiße Verbrennungsluft gelangt nun in die Mischzone, in der die flüssigen Edukte des Furnace Black Prozess eingesprüht werden und verdampfen. Die Rohstoffe werden auf $150-250{ }^{\circ} \mathrm{C}$ vorgewärmt, um das Zerstäuben der hochviskosen Flüssigkeiten in der Mischzone zu erleichtern. In der Reaktionszone kommt es dann zur Pyrolysereaktion, wobei die gewünschten Industrieruße und Wasserstoff entstehen. In der Reaktionszone herrschen Temperaturen von $1200{ }^{\circ} \mathrm{C}$ (grobe Ruße) bis $1900{ }^{\circ} \mathrm{C}$ (Pigmentruße).

Im Anschluss erfolgt das Abkühlen des Reaktionsgemisches (Quenchen) auf einen Temperaturbereich von 500-800 ${ }^{\circ} \mathrm{C}$. Hierzu wird Wasser in die Quenchzone eingesprüht, welches die Pyrolysereaktion vorzeitig beendet. Dementsprechend ist das Quenchen ein entscheidender Arbeitsschritt, um die Spezifikationen und gewünschten Eigenschaften des Produktes zu steuern. 


\subsection{Verankerung von RAFT-Agenzien auf der Rußoberfläche}

In diesem Kapitel wird der aktuelle Stand der Forschung kurz und knapp umrissen, um die literaturbekannten Ergebnisse vorzustellen. Aufgrund der vielfältigen Anwendungsmöglichkeiten von Industrierußen ergibt sich ein sehr breites Forschungsgebiet. Die Verbesserung der Mischungseigenschaften, die verbesserte Löslichkeit in organischen oder wässrigen Lösungsmitteln $\left.{ }^{[72,} 73\right]$ oder die bessere Dispergierbarkeit der Rußpartikel in einem Polymergemenge stehen bei diesen Arbeiten im Vordergrund ${ }^{[74-76]}$. Natürlich werden auch die verstärkenden Eigenschaften des Füllstoffes Ruß weiterhin untersucht und verbessert ${ }^{[77]}$. Die chemische Modifikation der Rußoberfläche ist allerdings immer ein zentrales Thema der aktuellen Forschung. Hierbei gibt es unterschiedliche Ansätze, um die gewünschten Eigenschaften zu erreichen. Aufgrund der strukturellen Ähnlichkeiten wird neben dem eigentlichen Ruß in diesem Kapitel auch auf die Kohlenstoff-Nanoröhrchen eingegangen. Auch auf diesem Gebiet gibt es interessante Ansätze und Überlegungen, um die Oberfläche dieses einzigartigen Materials chemisch zu modifizieren ${ }^{[78-84]}$.

Eine sehr effektive und einfache Form der Oberflächenmodifikation stellt die oxidative Behandlung des Rußes dar. Hierbei kann die chemische Modifikation mittels Salpetersäure ${ }^{[85]}$, mit Hilfe von Sauerstoffplasma ${ }^{[86]}$ oder auf elektrochemischen Wege erfolgen $^{[87]}$. Auch die katalytischen Wirkungen einiger Oxide ${ }^{[88]}$, welche im Oberflächenkontakt mit Ruß stehen, werden bei der Oxidation ${ }^{[89]}$ berücksichtigt. Neben dieser klassischen Oberflächenmodifikation, um die Löslichkeit und den Benetzungsgrad von Industrierußen in organischen Lösungsmitteln zu erhöhen, kann auch eine Anbindung von Polymeren an der Rußoberfläche die rheologischen Eigenschaften verbessern. Für diese Aufgabe gibt es ebenfalls verschiedene Herangehensweisen. Auf dem Gebiet der konventionellen radikalischen Polymerisation wäre die Anbindung einer Monomerfunktionalität an die Oberfläche denkbar. Das modifizierte Partikel kann dann im Verlauf der Polymerisation mit in die Kettenstruktur integriert werden ${ }^{[79]}$. Um diesen Gedankengang zu beenden, ist es natürlich auch möglich den Initiator an die Oberfläche der Kohlenstoff-Nanoröhrchen bzw. der Rußpartikel zu fixieren, um diese in einer radikalischen $^{[90]}$ oder anionischen ${ }^{[91]}$ Polymerisation $\mathrm{zu}$ verwenden. Aufgrund des ausgedehnten $\pi$-Elektronensystems in der Struktur des Rußes kann ein Radikal sehr lange und sehr gut an der Oberfläche stabilisiert werden. Dieser Effekt wird in der Literatur gerne als „Radikalfalle““92] bezeichnet. Für die Realisierung der „grafting to“ Methode 
ergibt sich nun die Möglichkeit, die bestehende Polymerkette mit einer Radikalfunktion zu bestücken und diese dann an der Rußoberfläche zu binden. Polymere, welche mit Hilfe des NMP-Mechanismus (vgl. Abbildung 2.4.2) hergestellt wurden, eignen sich für diese Art der Immobilisierung hervorragend. Nach Beendigung der Polymerisation wird in Gegenwart von Ruß das Polymer erhitzt und somit der homolytische Bindungsbruch herbeigeführt. Die entstehenden aktiven Spezies werden dann von der Rußoberfläche aufgenommen. Auch andere kontrollierte Polymerisationsarten können genutzt werden, um Polymer mit einer kontrollierten Kettenstruktur auf der Rußoberfläche zu binden. So konnten auch Initiatoren für die ATRP-Polymerisation ${ }^{[93,94]}$ auf der Oberfläche immobilisiert werden, wobei jedoch zuvor eine oxidative Behandlung des Rußes durchgeführt worden ist. Die erzwungene Erhöhung der Konzentration an Heteroatomen wird auch bei den bekannten RAFT-Polymerisationen an Rußpartikeln ${ }^{[95]}$ und Kohlenstoff-Nanoröhrchen ${ }^{[96,97]}$ durchgeführt.

Im Rahmen dieser Arbeit soll eine schnelle und effektive Möglichkeit gesucht bzw. untersucht werden, um eine RAFT-Polymerisation direkt von der Rußoberfläche zu ermöglichen. Es handelt sich in diesem Fall um eine „grafting from“ Methode, d.h. die Polymerketten wachsen direkt von der Oberfläche und werden nicht nach der Polymerisation auf die Partikel aufgebracht (,grafting to"). Die Anbindung des RAFTAgens erfolgt über die R-Gruppe (vgl. Abbildung 3.4.1), wodurch die RAFT-Gruppe während der Polymerisation von der Oberfläche ,,wegwandert“. Durch den R-GruppenAnsatz ergeben sich verschiedene Vorteile im Vergleich zur Anbindung über die ZGruppe. In Abbildung 8.5.1 werden die beiden Möglichkeiten der Immobilisierung von RAFT-Agenzien auf Oberflächen verdeutlicht. Der Hauptunterschied zwischen den beiden Varianten ist die Position der RAFT-Gruppe nach der Beendigung der Polymerisation. Beim Z-Gruppen-Ansatz verbleibt diese an der Oberfläche. Die Polymerdichte (Propfdichte) ist auf der Oberfläche geringer als für den R-GruppenAnsatz, da die Radikale während der Polymerisation an die RAFT-Gruppe gelangen müssen. Dieser Weg ist allerdings sterisch schon durch die bereits vorhandenen Polymerketten an der Oberfläche gehindert, zumal diese nicht wie in der schematischen Abbildung abstehen, sondern sich eher um das Partikel wickeln. Diese sterische Hinderung kann bei der Immobilisierung des RAFT-Agens über die R-Gruppe vermieden werden, da die „schlafende“ Spezies sich am Kettenende befindet. 

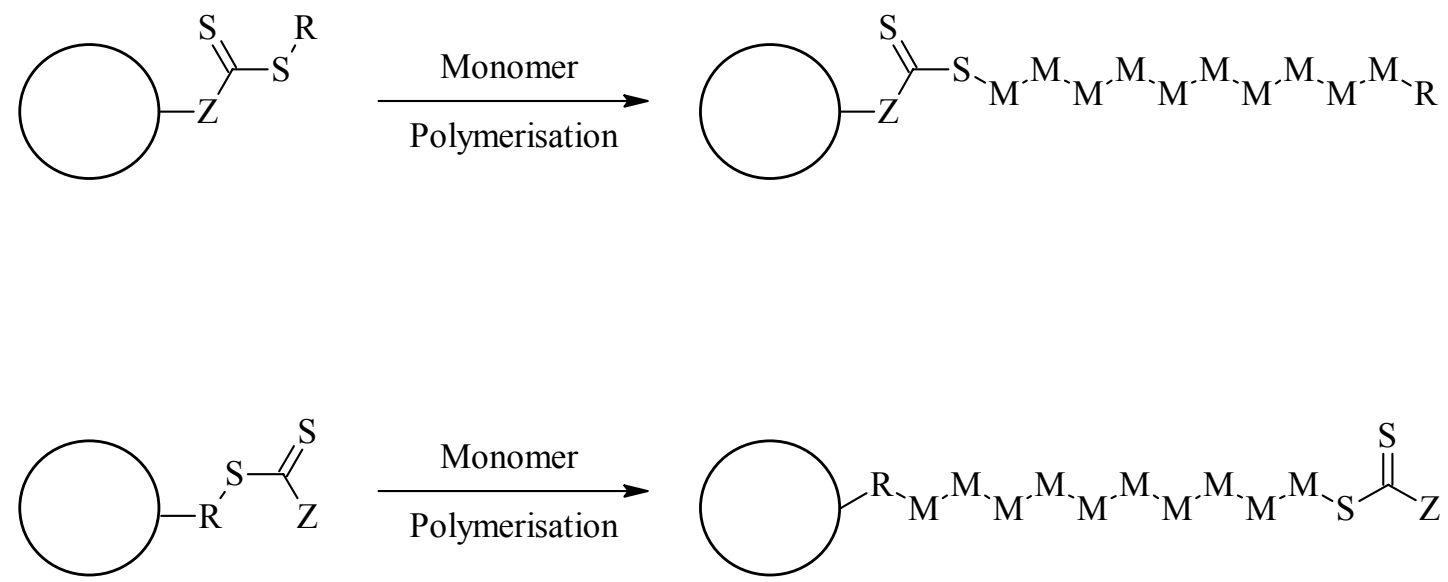

\section{Abbildung 8.5.1}

Schematische Darstellung der RAFT-Polymerisation von der Oberfläche: Die Verankerung des RAFT-Agens erfolgt im ersten Fall über die Z-Gruppe, wobei das untere Beispiel eine Immobilisierung über die R-Gruppe verdeutlicht. Die Monomereinheiten in den Polymerketten werden in diesem Beispiel durch ein „M“ symbolisiert. Der Begriff „Polymerisation“ unter den Reaktionspfeilen bezeichnet den Ablauf einer RAFT-Polymerisation mit den entsprechenden Komponenten (Initiator, Lösungsmittel, Comonomer usw.).

Die Anlagerung von Monomereinheiten im Wachstumsschritt an die „aktive“ Spezies verläuft beim R-Gruppe-Ansatz nicht an der Oberfläche sondern in Lösung. Aus diesem Grund befinden sich nach Beendigung der Polymerisation auch Polymerketten mit RAFTGruppen und kontrollierter Kettenstruktur in Lösung, welches bei der GPC-Analyse durch eine Absorption des UV-Signals im makromolekularen Bereich bestätigt wird. Diese Tatsache kann bei der späteren Auswertung berücksichtigt werden, da sich die Abspaltung der Polymerketten von Oberflächen für die Analyse teilweise schwierig gestaltet. Die „freien“ Polymerketten in Lösung besitzen eine vergleichbare Struktur, Zusammensetzung und einen identischen Polymerisationsgrad mit den Makromolekülen, welche an der Oberfläche gebunden sind. Die Abtrennung der gebildeten Polymerketten gestaltet sich beim Z-Gruppen-Ansatz leichter, da diese mit einem Überschuss an Radikalen abgespaltet werden können. Dieser Mechanismus wird für die RAFTPolymerisation an Silica-Oberflächen ${ }^{[98]}$ genutzt. Durch die hohe Radikalkonzentration wird die makromolekulare R-Gruppe aktiviert, um dann sofort mit einem anderen Radikal zu terminieren. Das RAFT-Agens kann bei geschickter Wahl der Radikalquelle wieder vollständig hergestellt werden. Auf diesem Weg kann ein farbloses und geruchsneutrales Polymer mit kontrollierter Kettenstruktur hergestellt werden, da die RAFT-Gruppen an 
der Oberfläche verbleiben. Was bei der Abtrennung vorteilhaft ist, kann allerdings für andere Anwendungen nachteilig sein. Bei der Vulkanisation könnte die schwache Schwefel-Kohlenstoff-Bindung gespalten werden und somit die Polymerkette ablösen. In diesem Fall wäre die Herstellung von Kautschuken mit direkter Anbindung an den Füllstoff sinnlos gewesen. Aus diesem Grund wird bei der RAFT-Polymerisation von Rußoberflächen der R-Gruppen-Ansatz favorisiert. Im schlimmsten Fall könnte bei der Vulkanisation eine Umwandlung der RAFT-Gruppe in ein Thiol erfolgen, welches jedoch keinen Einfluss auf die Anbindung an der Oberfläche hätte. In der Literatur ergeben sich verschiedene Lösungsansätze für die Verankerung von funktionellen Gruppen oder ganzen Molekülen an der Oberfläche von Industrierußen. Es stellt sich somit die Frage, welche Technik für die Verankerung der RAFT-Agenzien vorteilhaft ist.

Neben den zuvor beschriebenen Immobilisierungstechniken gibt es auch die Möglichkeit mittels Diels-Alder-Reaktionen ${ }^{[99-101]}$ neue Funktionalitäten an die Oberfläche zu binden. Die [4+2] Cycloaddition eines konjugierten Diens mit einer Doppel- oder Dreifachbindung (Dienophil) ist eine sehr bekannte und gleichwohl bedeutende Reaktion in der Organischen Chemie ${ }^{[102]}$. Die Möglichkeit der Durchführung einer Diels-AlderReaktion an Kohlenstoff-Nanoröhrchen ist seit $2002^{[103]}$ bekannt, wobei die Anbindung durch Mikrowellenstrahlung ${ }^{[104]}$, fluorierten Oberflächen ${ }^{[99]}$ oder Metallkomplexe ${ }^{[105]}$ erleichtert wird. Die Vorteile der Diels-Alder-Reaktion liegen in der Einfachheit der Oberflächenmodifikation. Es sind keine weiteren Edukte von Nöten, die Vorbehandlung mit oxidierenden Agenzien entfällt und die Reaktionsführung kann in einem Schritt abgeschlossen werden.

Aus diesen eben genannten Gründen ist ein RAFT-Agens mit einer Ankergruppe für eine Diels-Alder-Reaktion an der Oberfläche von Industrierußen vorteilhaft und sehr effektiv. 
a)

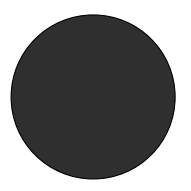<smiles>CCCSC(=S)SC(C)C(=O)OCc1ccco1</smiles><smiles>C=CC=C</smiles>

b)<smiles>CC(Br)C(=O)OCc1ccco1</smiles><smiles>[B]C(C)C(=O)OCC1=CC2C(C)CCCCCCCCCCCCCC2O1</smiles><smiles>CCCSC(=S)[S-][NH3+]</smiles>
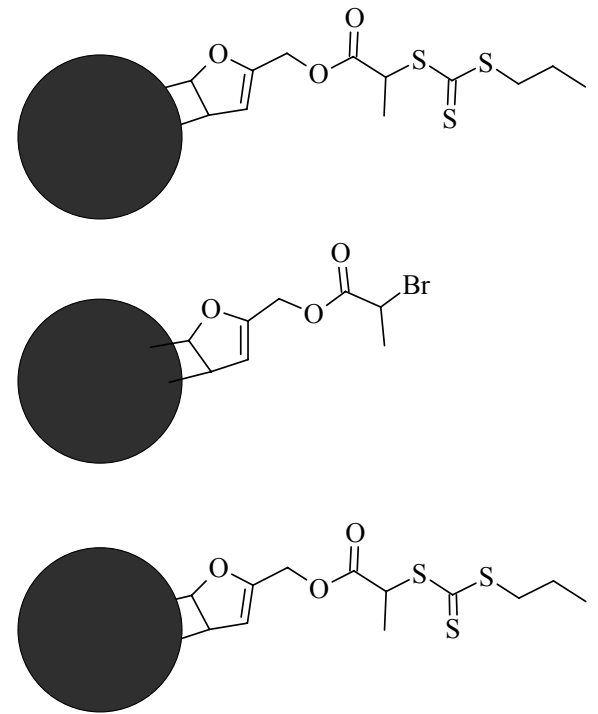

Abbildung 8.5.2

Schematische Darstellung der Verankerungsmechanismen von Furanderivaten an Rußpartikeln: Im Schema a) erfolgt die Immobilisierung in einem Reaktionsschritt und wird für einen Temperaturbereich bis ca. $T=100{ }^{\circ} \mathrm{C}$ angewandt. Das Schema b) verläuft in zwei Schritten und ermöglicht höhere Temperaturen für die Immobilisierung des Furanderivats. Der zweite Reaktionsschritt in b) kann dann bei Raumtemperatur oder leicht erhöhten Temperaturen $\left(T=50^{\circ} \mathrm{C}\right)$ durchgeführt werden. Als Lösungsmittel werden Dibenzylether (DBE) und Dimethylsulfoxid (DMSO) verwendet.

Im Rahmen dieser Arbeit werden Furanderivate als Ankergruppen verwendet, um RAFTAgenzien an die Rußoberfläche zu binden. In Abbildung 8.5.2 werden die Möglichkeiten der Immobilisierung verdeutlicht. Aufgrund der thermischen Instabilität der RAFTGruppen bei erhöhten Temperaturen kann die Verankerung auch in zwei Reaktionsschritten durchgeführt werden. Ob das Furanderivat in der Diels-Alder-Reaktion als Dien oder als Dienophil reagiert, kann nicht eindeutig geklärt werden. In der Literatur ${ }^{[106]}$ werden beide Möglichkeiten in Betracht gezogen.

\subsubsection{Abtrennung der Polymerketten von der Rußoberfläche}

Das Abtrennen der Polymerketten von der Rußoberfläche ist vorteilhaft, um diese chemisch charakterisieren $\mathrm{zu}$ können. Vor allem die Bestimmung der Molekulargewichtsverteilung mittels GPC oder die Analyse der Mikrostruktur des Polymers mittels NMR-Spektroskopie lassen sich in Gegenwart von Ruß nicht durchführen. Aus diesem Grund besitzen die Ankergruppen der RAFT-Agenzien, welche 
für die Polymerisation von der Rußoberfläche verwendet werden, funktionelle Gruppen, um eine gezielte Abspaltung zu ermöglichen. Allerdings sollten diese „Sollbruchstellen“ so stabil sein, dass die Polymerketten fest auf der Oberfläche fixiert sind und es zu keiner ungewollten thermischen oder chemischen Abspaltung kommt. Aus diesem Grund wird in dieser Arbeit erstmalig eine Esterfunktionalität in die Struktur der RAFT-Agenzien integriert, um eine nachträglich Abspaltung zu ermöglichen. Somit kann nach der Polymerisation eine saure oder basische Esterhydrolyse ${ }^{[107]}$ durchgeführt werden. Diese Möglichkeit der Abspaltung der Polymerketten nach der RAFT-Polymerisation von der Rußoberfläche ist bis jetzt nicht literaturbekannt. Zwischen den beiden Möglichkeiten der Hydrolyse besteht allerdings ein wichtiger Unterschied. Bei der sauren Esterhydrolyse fungiert die Säure als Katalysator und kann nach Bedarf wieder zurück gewonnen werden. Bei der basischen Esterhydrolyse ist die Lauge ein Reaktand und wird in äquimolaren Mengen verbraucht.

$$
\begin{array}{lll}
\mathrm{RCO}_{2} \mathrm{R}^{\prime}+\mathrm{H}_{2} \mathrm{O} & \stackrel{\mathrm{H}^{+}}{\rightleftharpoons} \mathrm{RCO}_{2} \mathrm{H}+\mathrm{R}^{\prime} \mathrm{OH} \\
\mathrm{RCO}_{2} \mathrm{R}^{\prime}+\mathrm{OH}^{-} \stackrel{\mathrm{H}_{2} \mathrm{O}}{\longrightarrow} \mathrm{RCO}_{2}^{-}+\mathrm{R}^{\prime} \mathrm{OH}
\end{array}
$$

\footnotetext{
Abbildung 8.5.3

Schematische Darstellung der sauren bzw. basischen Esterhydrolyse: Diese Reaktion wird für die Abtrennung der Polymerketten von der Rußoberfläche verwendet.
}

In Abbildung 8.5.3 ist das Schema der Esterhydrolyse dargestellt. Die säurekatalysierte Esterhydrolyse ist eine Gleichgewichtsreaktion. Dagegen ist die basische Esterpyrolyse nahezu irreversibel. Für die Abspaltung der Polymerketten an der Ankergruppe der RAFT-Agenzien wird die basische Esterhydrolyse bevorzugt, wobei hier eine 25\%ige Lösung von Natriummethanolat in Methanol verwendet wird. In Abbildung 8.5.4 ist die Abspaltung des Polymers mit den entsprechenden Chemikalien graphisch dargestellt. Die RAFT-Gruppen am Ende der Polymerketten werden höchstwahrscheinlich ebenfalls aufgespalten, so dass im Endeffekt ein Thiol als funktionelle Gruppe ausgebildet wird. 


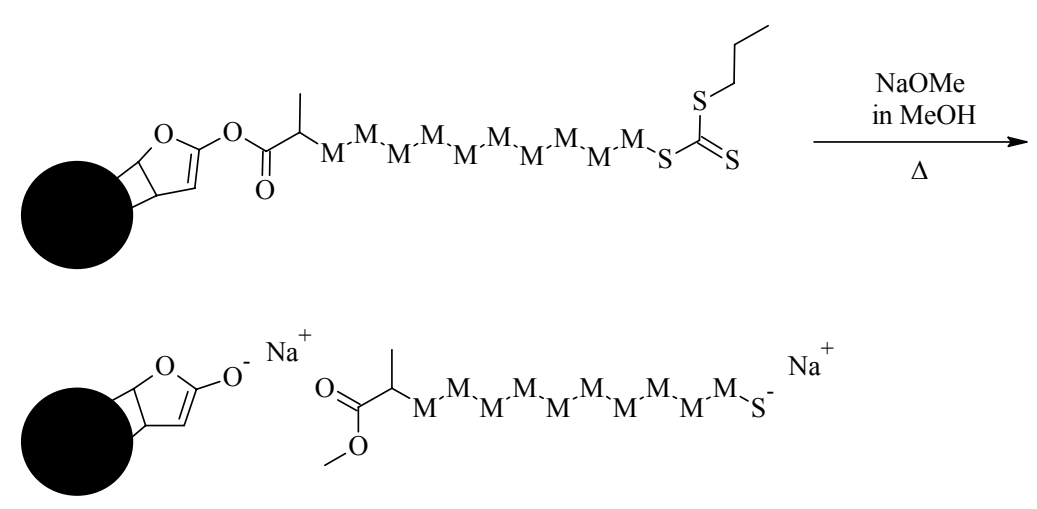

\section{Abbildung 8.5.4}

Schematische Darstellung der Abspaltung der Polymerkette durch eine basische Esterhydrolyse: Die Monomereinheiten in den Polymerketten werden in diesem Beispiel durch ein „M“ symbolisiert.

Für die Ankergruppe „FTBP“ mit der Thioesterfunktionalität ist auch eine Aminolyse denkbar, wodurch ebenfalls ein Thiol entstehen würde. Die Aminolyse (Abbildung 8.5.5) wird oft für das Abspalten der RAFT-Gruppen ${ }^{[108]}$ von der Polymerkette verwendet, um eine Entfärbung ${ }^{[109]}$ des Polymers zu erreichen.

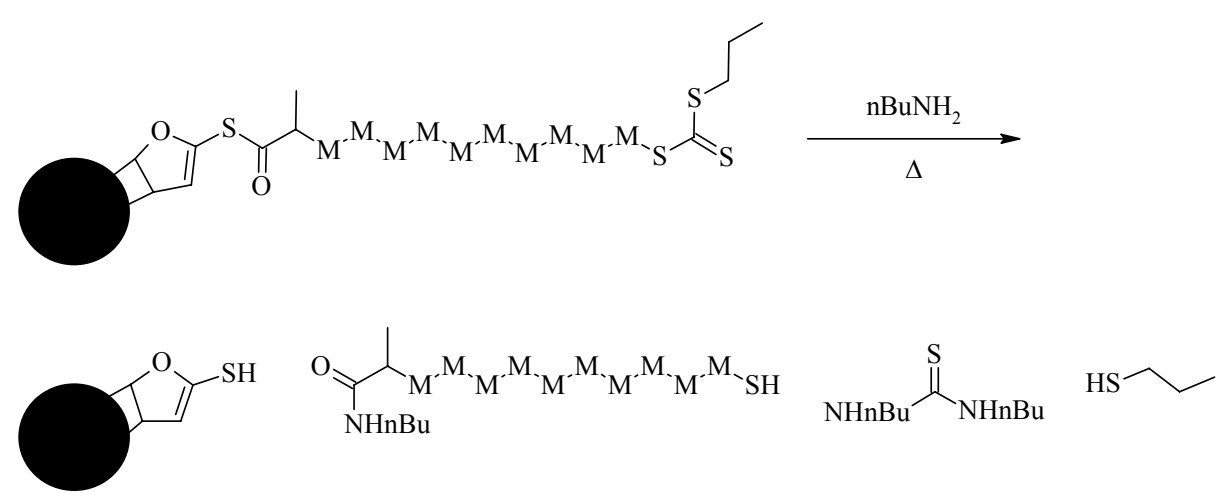

\section{Abbildung 8.5.5}

Schematische Darstellung der Abspaltung der Polymerkette durch Aminolyse mit n-Butylamin: Die Monomereinheiten in den Polymerketten werden in diesem Beispiel durch ein "M“ symbolisiert.

\subsubsection{Abtrennung des Polymers in Lösung vom Polymer an der Rußoberfläche}

In Kapitel 8.5 wurden bereits die Vor- und Nachteile des Z- und R-Gruppen-Ansatzes diskutiert. Im Rahmen der Untersuchungen werden für die Polymerisation von der Rußoberfläche RAFT-Agenzien über die R-Gruppe an das Füllmaterial gebunden. Im 
Verlauf der Polymerisation entsteht aufgrund des Reaktionsmechanismus auch Polymer in Lösung (PiL). Dieses muss von dem Polymer getrennt werden, welches an die Oberfläche gebunden worden ist (PaR), um eine Bestimmung der Beladungsdichte durch eine thermogravimetrische Analyse zu ermöglichen. Zwei Trennungswege sind denkbar und können je nach Menge der Rußpartikel eingesetzt werden. Die Extraktion im Soxhlet eignet sich für größere Massen an Ruß $(m>1 \mathrm{~g})$. Kleinere Mengen an Ruß konnten nach dem mehrstündigen Waschen nicht mehr aus der Extraktionshülse isoliert werden, da die Partikel in die Poren aufgenommen worden sind. Als praktikablere Lösung hat sich das Aufarbeiten in der Zentrifuge bewährt. Hierzu wird die Probe nach der Umsatzbestimmung in ein Zentrifugenröhren überführt $(V=30 \mathrm{ml})$ und mit Tetrahydrofuran überschichtet. Danach werden die Rußpartikel für 12 Stunden unter Rühren in Lösung gebracht. Die Zentrifuge wird auf eine Temperatur von $T=20^{\circ} \mathrm{C}$ temperiert. Die Proben werden für 45 Minuten bei $15300 \mathrm{U} / \mathrm{min}$ zentrifugiert. Danach wird die überstehende Lösung abgenommen und das darin gelöste Polymer in Methanol ausgefällt. Die Rußpartikel werden wieder mit Tetrahydrofuran überschichtet und für 12 Stunden gerührt. Dieser Vorgang wird solange wiederholt, bis aus der überstehenden Lösung kein Polymer mehr ausgefällt werden kann (Abbildung 8.5.6). In der Regel wurde diese Prozedur dreimal durchgeführt, um das Polymer in Lösung von den Rußpartikeln zu trennen.

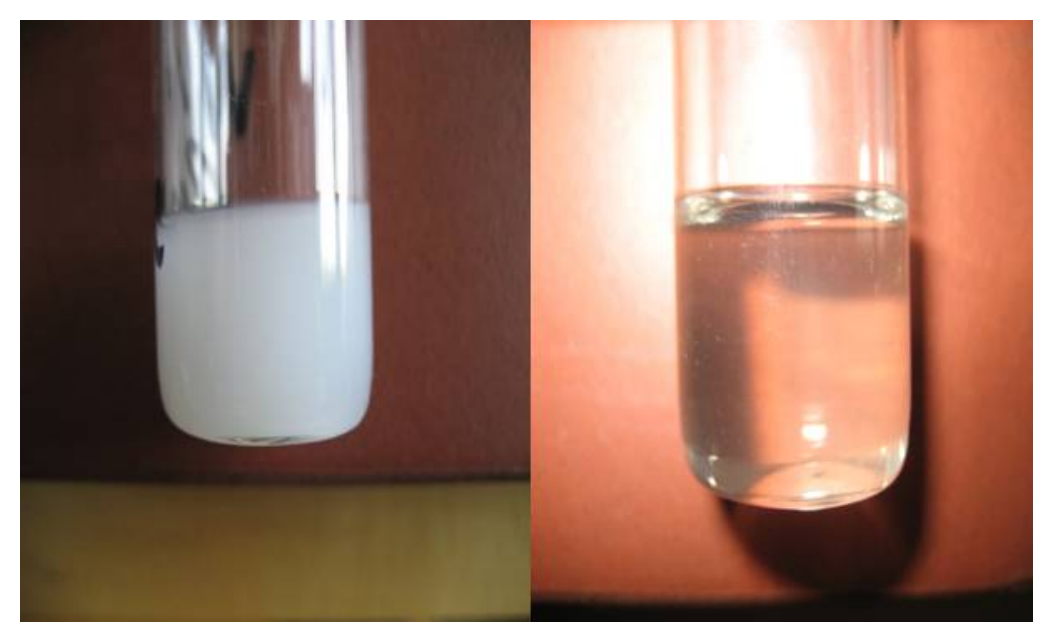

\footnotetext{
Abbildung 8.5.6

Ausfällung des Polymers in Lösung (PiL) der überstehenden Lösung in Methanol: Links vor der Aufarbeitung durch die Zentrifuge und rechts nach dreimaligem Zentrifugieren.
} 
Das Waschen im Soxhlet wurde vor allem bei der Verankerung der RAFT-Agenzien genutzt (Kapitel 8.6).

\subsection{Quantitative Analyse der Verankerung}

Die Verankerung der Furanderivate über eine Diels-Alder-Reaktion an die Oberfläche von Industrierußen wird durch Elementaranalyse und durch thermogravimetrische Messungen (TGA-Messungen) quantifiziert. Somit kann die Beladungsdichte $\rho_{\text {Agens }}$ bestimmt werden. Der Ruß wurde vor der weiteren Verwendung im Soxhlet mit destilliertem Wasser gewaschen (24 Stunden) und anschließend für weitere 24 Stunden bei $T=100{ }^{\circ} \mathrm{C}$ im Hochvakuum getrocknet. Obwohl der Industrieruß unterschiedslos vorbehandelt ist, variieren die Ergebnisse in der Bestimmung des Massenverlusts der TGA-Messung. So ergab die Messung ein und derselben Probe am gleichen experimentellen Setup an unterschiedlichen Tagen auch verschiedene Ergebnisse in der thermogravimetrischen Analyse. In Tabelle 8.6.1 sind beispielhaft die Analyseergebnisse einer Rußprobe aufgelistet. Auffällig sind hierbei die starken Schwankungen des Massenverlustes, obwohl es immer ein und dieselbe Probe war. Proben, welche bei der thermogravimetrischen Messung ein größeres Temperaturintervall durchlaufen, sollten auch einen größeren Massenverlust aufweisen.

Tabelle 8.6.1

Auflistung der Massenverluste des vorbehandelten Industrierußes: Ein und dieselbe Probe wurde an verschiedenen Tagen über einen Zeitraum von einem halben Jahr mit Hilfe der thermogravimetrischen Analyse vermessen. Die Proben wurden immer von Raumtemperatur (RT) bis zur angegebenen Höchsttemperatur unter Inertgasatmosphäre erhitzt und der Massenverlust ( $\left.m_{\text {Verlust }}\right)$ in Gewichtsprozent ermittelt.

\begin{tabular}{|c|c|c|}
\hline Probe & $T_{\text {Intervall }}$ in ${ }^{\circ} \mathrm{C}$ & $m_{\text {Verlust }}$ in $\mathrm{w} \%$ \\
\hline \multirow{4}{*}{ Ruß } & RT-500 & 2,47 \\
\cline { 2 - 3 } & RT-700 & 8,34 \\
\cline { 2 - 3 } & RT-800 & 9,42 \\
\cline { 2 - 3 } & RT-800 & 0,44 \\
\cline { 2 - 3 } & RT-1400 & 7,23 \\
\hline
\end{tabular}

Diese Annahme wird auch anfänglich erfüllt. Allerdings zeigen die Messungen bis $T=800{ }^{\circ} \mathrm{C}$ einen widersprüchlichen Verlauf. Anscheinend spielen Umwelteinflüsse, wie Luftfeuchtigkeit und Luftdruck, eine Rolle und können das Ergebnis signifikant 
beeinflussen. Die thermogravimetrische Analyse scheint somit nur bedingt geeignet, um den Verlauf der Immobilisierung quantitativ zu beschreiben. Aus diesem Grund wird für die Bestimmung der Beladungsdichte an Ankergruppen bzw. RAFT-Agenzien die Elementaranalyse bevorzugt. Um die Beladungsdichte an der Rußoberfläche zu bestimmen, wird bei der Elementaranalyse der prozentuale Anteil an Schwefelatomen in der Probe bestimmt. Der Schwefelgehalt des unbehandelten Rußes ist so klein, dass eine Bestimmung innerhalb der Kalibrierung nicht möglich ist. Aus diesem Grund wird für den unbehandelten Ruß ein Wert von Null angenommen. Da der Schwefelgehalt der Ankergruppen bzw. der RAFT-Agenzien bekannt ist, kann somit die Beladungsdichte $\rho_{\text {Agens }}$ berechnet werden. Die thermogravimetrische Analyse wurde jedoch bei allen Proben durchgeführt, um einen zweiten Messwert zu erhalten. Um die zuvor beschriebenen Schwankungen auszugleichen, ist es sinnvoll neben der $\mathrm{zu}$ messenden Probe auch am selben Tag immer den vorbehandelten Ruß als Referenz zu messen. Da die beiden Proben den gleichen äußeren Bedingungen unterliegen, kann durch die Differenz der jeweiligen Massenverluste die theoretische Beladungsdichte bestimmt werden. Die so erhaltenen Ergebnisse stimmen in vielen Fällen mit den Ergebnissen der Elementaranalyse überein. Die Hauptanwendung der thermogravimetrischen Messung liegt allerdings in der Bestimmung des Massenverlustes an Polymer, welches an der Oberfläche gebunden ist. Füllstoffe mit Polystyrol an der Oberfläche zeigen einen charakteristischen starken Massenabfall in einem Temperaturbereich von $T=300-500{ }^{\circ} \mathrm{C}$ (Abbildung 8.6.1). 


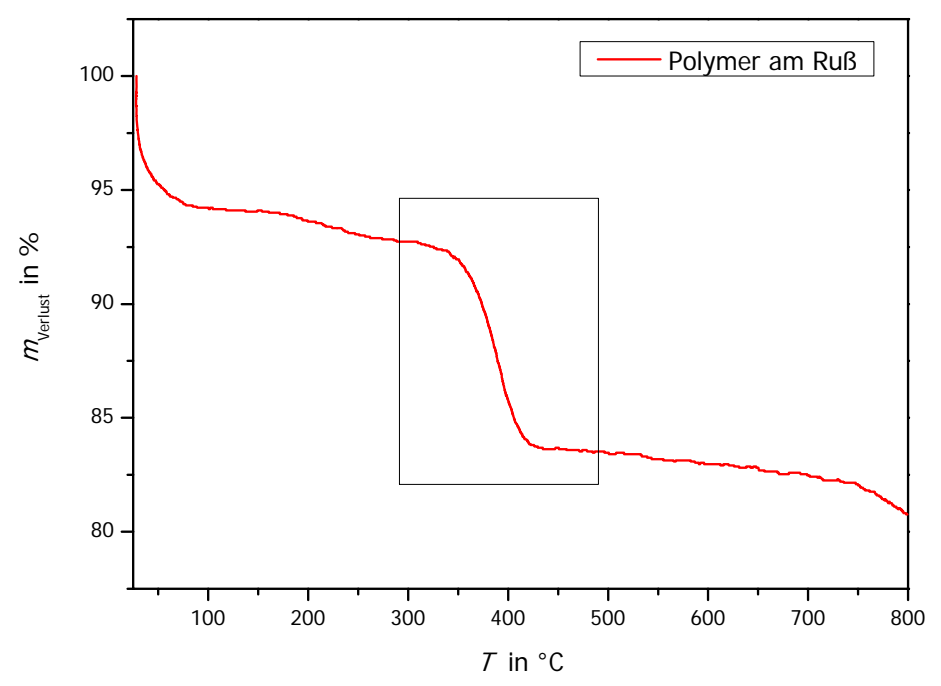

\section{Abbildung 8.6.1}

Darstellung eines Kurvenverlaufs der thermogravimetrischen Analyse einer Rußprobe mit Polystyrol an der Oberfläche: Der Massenverlust zwischen Raumtemperatur und $T=300{ }^{\circ} \mathrm{C}$ ist teilweise sehr unterschiedlich und wird deshalb in der Auswertung nicht berücksichtigt. Der umrahmte Bereich ist für die Ablösung des Polymers von der Oberfläche relevant.

In Tabelle 8.6.2 sind die Ankergruppen bzw. RAFT-Agenzien, welche für die Immobilisierung verwendet werden, zur Übersicht in der Strukturformel abgebildet.

\section{Tabelle 8.6.2}

Übersicht der verwendeten Ankergruppen bzw. RAFT-Agenzien:

\begin{tabular}{|c|c|}
\hline Name & Strukturformel \\
\hline FTBP & \\
\hline FABP & \\
\hline FTВРТTCP & \\
\hline FABРTTCP & \\
\hline
\end{tabular}


In der Tabelle 8.6.3 sind die Ergebnisse der Immobilisierung mit der Ankergruppe „FTBP“ dargestellt. Das Stoffmengenverhältnis von Ruß und Ankergruppe liegt bei 10:1, wobei hierbei für den Industrieruß eine Molmasse von $M=12 \mathrm{~g} / \mathrm{mol}$ angenommen wird. Als Lösungsmittel wird immer Dibenzylether (DBE) verwendet, da dieser eine gute Löslichkeit für alle verwendeten Komponenten hat und einen hohen Siedepunkt aufweist. Die Proben werden für die angegebenen Zeiten erhitzt und dann für mindestens 12 Stunden in einem Soxhlet mit Tetrahydrofuran gewaschen.

Tabelle 8.6.3

Auflistung typischer Ergebnisse der Immobilisierung mit der Ankergruppen „FTBP" auf der Rußoberfläche: Die Stoffmengenverhältnisse von $n_{\mathrm{Ru}}: n_{\mathrm{FTB}}: n_{\mathrm{DBE}}$ sind in diesem Fall 10:1:22.

\begin{tabular}{|c|c|c|c|}
\hline Name & $T$ in ${ }^{\circ} \mathrm{C}$ & $t$ in $\mathrm{h}$ & $\rho_{\text {Agens }}$ in $\mathrm{mmol} / \mathrm{g}$ \\
\hline FTBP01 & 100 & 21 & 0,284 \\
\hline FTBP02 & 130 & 12 & 0,356 \\
\hline FTBP03 & 170 & 13 & 0,263 \\
\hline
\end{tabular}

Der Anstieg des Schwefelgehaltes an der Rußoberfläche ist nach der Immobilisierung signifikant und beweist, dass die Verankerung über eine Diels-Alder-Reaktion möglich ist. In den Ergebnissen der Beladungsdichten in Tabelle 8.6.3 spiegelt sich der Gleichgewichtscharakter der Verankerungsreaktion wider. Bei höheren Umsetzungstemperaturen steigen zunächst die Beladungsdichten an, um dann bei noch höheren Temperaturen wieder abzunehmen. Es scheint also ein Temperaturoptimum für die Verankerung der Furanderivate an der Rußoberfläche zu geben. Die Ergebnisse der Immobilisierung stimmen sehr gut mit anderen Arbeiten ${ }^{[110]}$ überein.

Im folgenden Abschnitt werden die Reaktionszusammensetzungen und die Ergebnisse der Analyse von einigen modifizierten Rußproben in tabellarischer Form wiedergegeben.

Tabelle 8.6.4

Auflistung des Ergebnisses der Immobilisierung mit der Ankergruppen „FABPTTCP“ auf der Rußoberfläche: Die Stoffmengenverhältnisse von $n_{\mathrm{Ru}}: n_{\mathrm{FABPTTCP}}: n_{\mathrm{DBE}}$ sind in diesem Fall 10:1:30.

\begin{tabular}{|c|c|c|c|}
\hline Name & $T$ in ${ }^{\circ} \mathrm{C}$ & $t$ in $\mathrm{h}$ & $\rho_{\text {Agens }}$ in $\mathrm{mmol} / \mathrm{g}$ \\
\hline FABPTTCP01 & 100 & 24 & 0,118 \\
\hline
\end{tabular}

Die Immobilisierung, welche in den Tabellen 8.6.5 und 8.6.6 beschrieben wird, wird erstmalig in zwei Reaktionsschritten durchgeführt, um eine höhere Beladungsdichte zu 
erzielen. Im „1.Schritt“ wird hierbei nur die Ankergruppe aufgebracht und dann die Beladungsdichte ermittelt. Die Umsetzung zum RAFT-Agens erfolgt in DMSO unter verschiedenen Reaktionsbedingungen.

Tabelle 8.6.5

Auflistung des Ergebnisses der Immobilisierung mit der Ankergruppen „FABP“ (1,0 eq.) (1. Schritt) und der anschließenden Umsetzung mit „TTCP“ (1,1 eq.) auf der Rußoberfläche: Die Stoffmengenverhältnisse von $n_{\mathrm{Ruß}}: n_{\mathrm{FABP}} n_{\mathrm{DBE}}$ sind in diesem Fall 42:1:80. Für die Umsetzung mit „TTCP“ beträgt das Stoffmengeverhältnis $n_{\text {TTCP: }} n_{\text {DMSO }}$ gleich 1:330.

\begin{tabular}{|c|c|c|c|}
\hline Name & $T$ in ${ }^{\circ} \mathrm{C}$ & $t$ in $\mathrm{h}$ & $\rho_{\text {Agens }}$ in $\mathrm{mmol} / \mathrm{g}$ \\
\hline 1.Schritt & 130 & 48 & - \\
\hline FABPTTCP02 & 50 & 20 & 0,192 \\
\hline
\end{tabular}

Es ist gut zu erkennen, dass die Beladungsdichte für die Verankerungsreaktion in Tabelle 8.6.5 größer ist als zuvor, obwohl sich die Stoffmenge der Ankergruppe bezogen auf die Rußpartikel verkleinert hat und die Lösung stärker verdünnt war. In Hinblick auf die Herstellung von Proben in einem Maßstab von $m=100 \mathrm{~g}$ mit einem Füllstoffanteil von 50 Massenprozent wird versucht, eine optimale Zusammensetzung und einen effektiven Temperaturverlauf $\mathrm{zu}$ finden, um eine hohe Beladungsdichte mit einem geringen Verbrauch an Verankerungsagenzien zu kombinieren. Aus diesem Grund werden die Reaktionsbedingungen in einem weiten Bereich variiert, was die Vergleichbarkeit der einzelnen Ergebnisse erschwert. Es hat sich leider gezeigt, dass die Umsetzung im „2. Reaktionsschritt“ nicht quantitativ abläuft. Um die minimale Konzentration an RAFTAgens zu bestimmen, wird der Massengehalt an Schwefel der modifizierten Rußprobe nach jeder Reaktion bestimmt. Aus der Differenz der beiden Werte, wird die Beladungsdichte für das RAFT-Agens ermittelt. In Tabelle 8.6.6 ist dieser Wert mit $\rho_{\text {Agens }}=0,035 \mathrm{mmol} / \mathrm{g}$ beziffert. 
Tabelle 8.6.6

Auflistung des Ergebnisses der Immobilisierung mit der Ankergruppen „FTBP“ (1,0 eq.) und der anschließenden Umsetzung mit „TTCP“ (1,1 eq.) auf der Rußoberfläche: Die Stoffmengenverhältnisse von $n_{\mathrm{Ru}}: n_{\mathrm{FTB}}: n_{\mathrm{DBE}}$ sind in diesem Fall 50:1:114. Für die Umsetzung mit „TTCP“ sind die Stoffmengeverhältnisse $n_{\mathrm{TTCP}}: n_{\mathrm{DMSO}}$ gleich 1:330.

\begin{tabular}{|c|c|c|c|}
\hline Name & $T$ in ${ }^{\circ} \mathrm{C}$ & $t$ in $\mathrm{h}$ & $\rho_{\text {Agens }}$ in $\mathrm{mmol} / \mathrm{g}$ \\
\hline 1.Schritt & 130 & 24 & 0,219 \\
\hline FTBPTTCP03 & 25 & 96 & 0,035 \\
\hline
\end{tabular}

Es ist allerdings festzuhalten, dass die Verankerung von RAFT-Agenzien über eine DielsAlder-Reaktion sehr erfolgreich abläuft. Die Beladungsdichten variieren in einem Bereich der ebenfalls für eine erfolgreiche Polymerisation von Kieselsäure ${ }^{[23,63,98, ~ 111-115] ~}$ verwendet wird. Die modifizierten Rußpartikel werden in Lösungsmittelpolymerisationen von Styrol in Toluol verwendet. Aus diesem Grund wurde eine Probe in Toluol für $t=24 \mathrm{~h}$ bei $T=87^{\circ} \mathrm{C}$ gerührt. Diese Parameter entsprechen den Reaktionsbedingungen der Polymerisation. Es soll geklärt werden, ob es zu einer messbaren Abnahme der Beladungsdichte kommt. Der Vergleich der Ergebnisse der Elementaranalyse vor und nach dem Experiment zeigt keine signifikante Veränderung in der Beladungsdichte. Es kann also davon ausgegangen werden, dass die RAFT-Agenzien durch den thermischen Einfluss der Polymerisation nicht abgelöst werden können.

Tabelle 8.6.7

Auflistung des Ergebnisses der Immobilisierung mit der Ankergruppen „FTBP“ $(1,0$ eq.) und der anschließenden Umsetzung mit „TTCP“ $(1,1$ eq.) auf der Rußoberfläche: Die Stoffmengenverhältnisse von $n_{\mathrm{Ru}}: n_{\mathrm{FTBP}}: n_{\mathrm{DBE}}$ sind in diesem Fall 39:1:97. Für die Umsetzung mit „TTCP“ sind die Stoffmengeverhältnisse $n_{\text {TTCP: }} n_{\text {Dмso }}$ gleich 1:120.

\begin{tabular}{|c|c|c|c|}
\hline Name & $T$ in ${ }^{\circ} \mathrm{C}$ & $t$ in $\mathrm{h}$ & $\rho_{\text {Agens }}$ in $\mathrm{mmol} / \mathrm{g}$ \\
\hline 1.Schritt & 100 & 48 & 0,256 \\
\hline FTBPTTCP04 & 50 & 24 & 0,132 \\
\hline
\end{tabular}




\subsection{Konventionelle radikalische Polymerisationen in Gegenwart von Ruß}

\subsubsection{Polymerisationen unter Sauerstoff}

In diesem Abschnitt wird der Einfluss von Industrierußen auf die konventionelle radikalische Polymerisation von Styrol untersucht. Die Polymerisationen werden unter Luftsauerstoff nach der Variante 1 (vgl. Kapitel 6.4.1) durchgeführt. Als Untersuchungsschwerpunkte werden die Umsatz-Zeit-Verläufe und die Entwicklung der mittleren Molekulargewichte mit dem Umsatz betrachtet, um eine mögliche Retardierung der Polymerisationsgeschwindigkeit ${ }^{[116]} \mathrm{zu}$ beobachten. In diesen Experimenten wird eine Initiatorkonzentration von $\quad c_{0}(\mathrm{ACC})=0,100 \mathrm{Mol} \% \quad$ angenommen. $\quad$ Das Stoffmengenverhältnis von Styrol und Toluol ist 1:2. Die Rußanteile werden variiert. Durch diesen Umstand ändert sich das Verhältnis von Monomer und Initiator zueinander nicht, auch wenn unterschiedliche Mengen an Ruß hinzugegeben werden. Somit kann der Einfluss des Industrierußes direkt ermittelt werden. In der experimentellen Umsetzung bedeutet dies, dass jeweils drei identische Polymerisationsansätze hergestellt werden, denen dann unterschiedliche Rußanteile zugemischt werden. Dadurch kann eine quantitative Aussage getroffen werden, in wieweit sich die Anteile des Rußes prozentual zum Gesamtsystem verändert haben. Die Umsatzbestimmung erfolgte gravimetrisch. Nach Beendigung der Polymerisation werden die Rußpartikel von der Polymerisationslösung durch Filtration getrennt. Die Rückstände im Filter werden mehrmals mit THF gewaschen. 


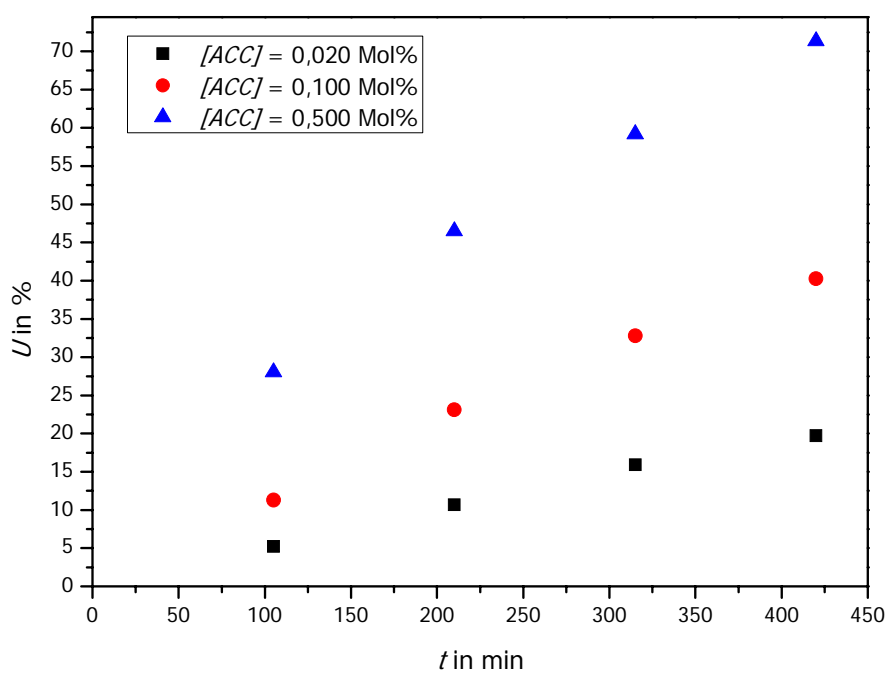

\section{Abbildung 8.7.1}

Darstellung der Umsatz-Zeit-Verläufe für eine konventionelle radikalische Polymerisation mit unterschiedlichen Initiatorkonzentrationen.

Der Umsatz-Zeit-Verlauf in Abbildung 8.7.1 entspricht den theoretischen erwarteten Werten (vgl. Gleichung 3.2.2) der konventionellen radikalischen Polymerisation. Bei einer Verfünffachung der effektiven Initiatorkonzentration steigt der Umsatz in der gleichen Zeit um den Faktor 2,23. Die Umsätze steigen stetig mit der Reaktionszeit. In den Abbildung 8.7.2 und 8.7.3 ist eine deutliche Abnahme der Polymerisationsgeschwindigkeit zu erkennen, wenn die Rußanteile erhöht werden. Die Umsätze fallen bei gleichbleibender Initiatorkonzentration ab. 


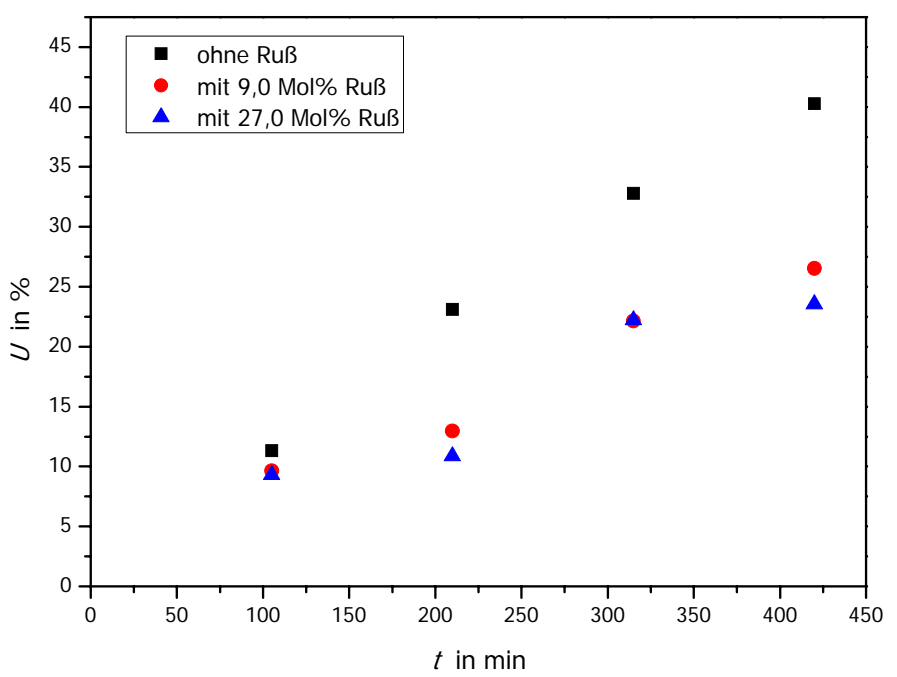

Abbildung 8.7.2

Darstellung der Umsatz-Zeit-Verläufe für eine konventionelle radikalische Polymerisation mit einer Initiatorkonzentrationen von $c_{0}(A C C)=0,100 \mathrm{Mol} \%$ für unterschiedliche Rußanteile: Die prozentualen Angaben der Rußanteile beziehen sich auf das Gesamtsystem.

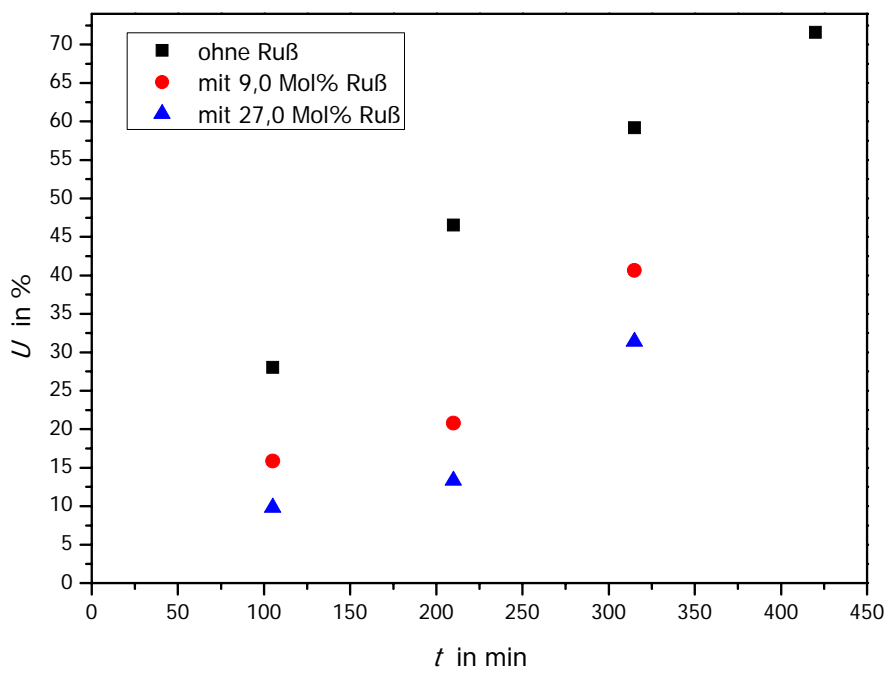

\section{Abbildung 8.7.3}

Darstellung der Umsatz-Zeit-Verläufe für eine konventionelle radikalische Polymerisation mit einer Initiatorkonzentrationen von $c_{0}(A C C)=0,500 \mathrm{Mol} \%$ für unterschiedliche Rußanteile: Die prozentualen Angaben der Rußanteile beziehen sich auf das Gesamtsystem. 
Es kann also davon ausgegangen werden, dass die effektive Initiatorkonzentration zum Beginn der Polymerisation stark herabgesetzt wird. Die gebildeten Radikale werden von den Rußpartikeln ,,abgefangen“ und fehlen somit in der Anfangsphase der Polymerisation. Nachdem die Rußoberfläche abgesättigt ist, verläuft die Polymerisation nach dem kinetischen Schema der konventionellen radikalischen Polymerisation ohne Rußpartikel. Die Polymerisation wird nach diesen Überlegungen nur in der Anfangsphase inhibiert. Die Molekulargewichte der Polymere müssten demnach nicht durch die Zugabe von Ruß beeinträchtigt werden. In den Abbildung 8.7.4 und 8.7.5 sind die mittleren Molekulargewichte gegen den Umsatz aufgetragen. Der Verlauf deutet eindeutig auf eine konventionelle radikalische Polymerisation hin. Die mittleren Molekulargewichte pendeln um einen Mittelwert und zeigen keine Abhängigkeit von der Rußkonzentration.

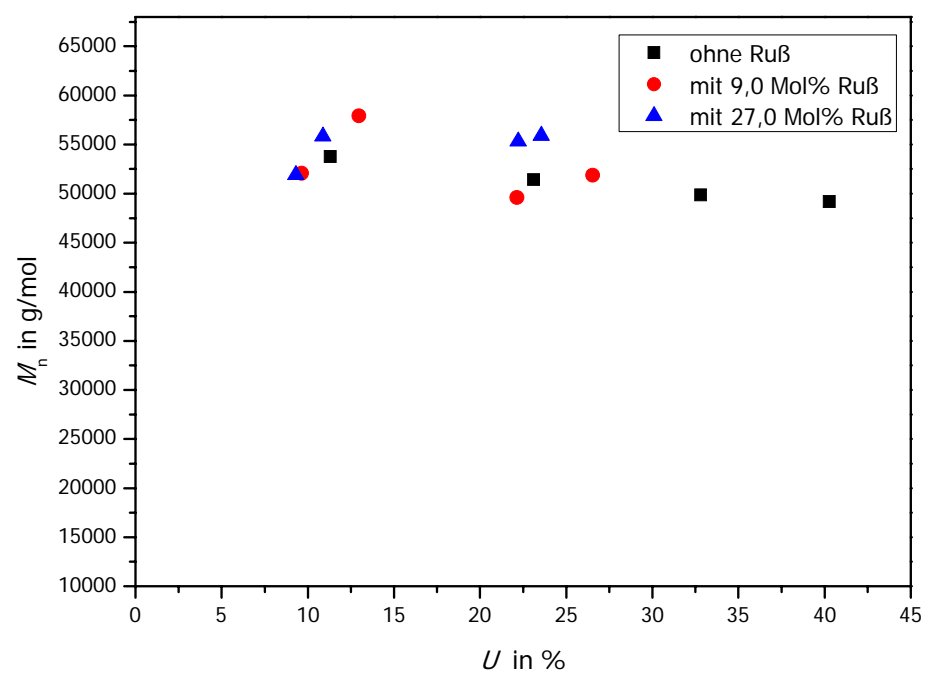

\footnotetext{
Abbildung 8.7.4

Auftragung der mittleren Molekulargewichte gegen den Umsatz mit unterschiedlichen Rußanteilen des Polymerisationsansatzes: Die Initiatorkonzentration beträgt $c_{0}(A C C)=0,100 \mathrm{Mol} \%$.
} 


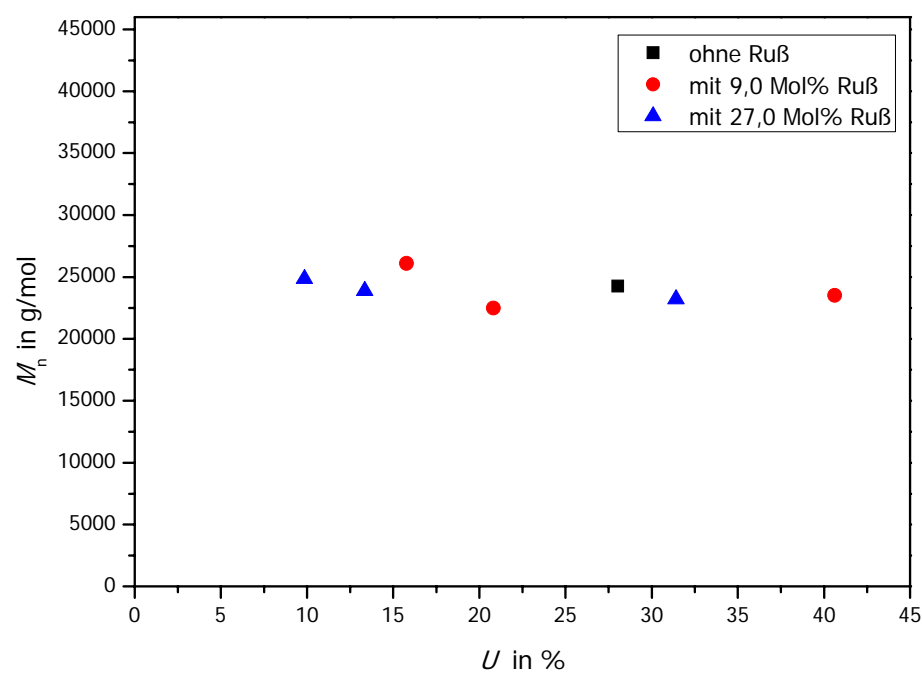

Abbildung 8.7.5

Auftragung der mittleren Molekulargewichte gegen den Umsatz mit unterschiedlichen Rußanteilen des Polymerisationsansatzes: Die Initiatorkonzentration beträgt $c_{0}(A C C)=0,500 \mathrm{Mol} \%$.

\subsubsection{Polymerisationen unter Argon-Atmosphäre}

In Kapitel 8.7.1 konnte gezeigt werden, dass die konventionelle radikalische Polymerisation in Gegenwart von Ruß durchgeführt werden kann, wobei die Umsätze mit höheren Rußanteilen nach gleicher Zeit immer kleiner werden. Die Molekulargewichte verändern sich dagegen kaum und sind mit denen der konventionellen radikalischen Polymerisation ohne Ruß vergleichbar. In diesem Kapitel soll nun der Einfluss von einer Luftsauerstoffatmosphäre auf den Polymerisationsmechanismus geklärt werden. Hierzu werden die Monomere, das Lösungsmittel, der Ruß und der Initiator einem dreimaligen „freeze-pump-and-thaw“-Zyklus unterzogen. Danach wird diese in eine „Glovebox“ der

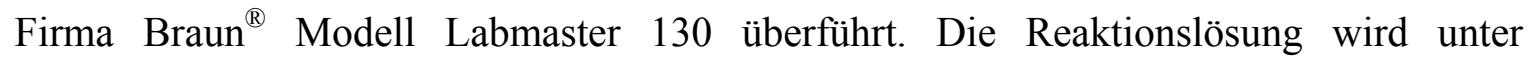
Argonatmosphäre nach Variante 2 zur Polymerisation gebracht. Aufgrund des geringen Einflusses des Industrierußes auf die Molekulargewichte der konventionellen radikalischen Polymerisation werden hier nur die Umsätze verglichen. Die Konzentration der Rußpartikel wird in Anlehnung an die beabsichtigten RAFT-Polymerisationen von der Oberfläche so gewählt, dass eine Beladungsdichte von $\rho_{\text {Agens }}=0,035 \mathrm{mmol} / \mathrm{g}$ erreicht wird. Diese Beladungsdichte ist mit der modifizierten Rußprobe „FTBPTTCP03“ 
identisch (vgl. Tabelle 8.6.6). Hierbei ist zu beachten, dass die Initiatorkonzentration und RAFT-Agens-Konzentration immer gleich groß sind, wodurch sich eine theoretische Beladungsdichte ableiten lässt. In Tabelle 8.7.1 ist eine typische Zusammensetzung des Polymerisationsansatzes aufgeführt.

Tabelle 8.7.1

Typische Zusammensetzung des Polymerisationsansatzes der konventionellen radikalischen Polymerisation unter Luftsauerstoff bzw. unter Argonatmosphäre:

\begin{tabular}{|c|c|c|}
\hline Initiator & ACC & $0,020 \mathrm{Mol} \%$ \\
\hline Monomer & Styrol & $32,2 \mathrm{Mol} \%$ \\
\hline Lösungsmittel & Toluol & $32,2 \mathrm{Mol} \%$ \\
\hline Feststoff & Ruß & $35,5 \mathrm{Mol} \%$ \\
\hline
\end{tabular}

In Tabelle 8.7.2 sind die Ergebnisse der gravimetrischen Umsatzbestimmung aufgelistet. Die Polymerisationen an Luftsauerstoff wurden zweimal durchgeführt und die Ergebnisse werden mit $U_{\text {Luft }}^{1}$ und $U_{\text {Luft }}^{2}$ bezeichnet. In diesem Fall wurde auf eine Abtrennung der Rußpartikel verzichtet, wodurch die Umsatzbestimmung nicht so stark fehlerbehaftet ist. Bemerkenswert ist der Anstieg der Umsätze bei den Polymerisationen unter Luftsauerstoff im direkten Vergleich zu denen, welche unter Argonatmosphäre durchgeführt wurden. Das Entgasen eines Polymerisationsansatzes soll den störenden Einfluss des Luftsauerstoffs als Biradikal auf die radikalische Polymerisation unterbinden, wodurch höhere Umsätze erzielt werden können.

Tabelle 8.7.2

Auflistung der erreichten Umsätze für verschiedene Initiatorkonzentrationen der konventionellen radikalischen Polymerisation unter Luftsauerstoff bzw. unter Argonatmosphäre:

\begin{tabular}{|c|c|c|c|}
\hline$c_{\mathrm{I}}$ in Mol\% & $U_{\text {Argon }}$ in \% & $U_{\text {Luft }}^{l}$ in \% & $U_{\text {Luft }}^{2}$ in \% \\
\hline 0,020 & 61,09 & 72,81 & 74,79 \\
\hline 0,040 & 69,76 & 76,52 & 78,92 \\
\hline
\end{tabular}

Anscheinend wird diese gewünschte Verbesserung des Schutzgases in Gegenwart von $\mathrm{Ruß}$ durch einen anderen Effekt überlagert. In der Literatur $^{[95]}$ werden die $\mathrm{zu}$ untersuchenden Polymerisationssysteme meistens unter Schutzgasatmosphäre zur Reaktion gebracht, wodurch dieser Effekt nicht in Erscheinung tritt. Industrieruße 
besitzen ein sehr hohes Adsorptionsvermögen von Gasen und Flüssigkeiten. Es ist also vorstellbar, dass während der Entgasung des Lösungsmittels die zuvor adsorbierten Gasmoleküle in den Poren der Rußpartikel entfernt worden sind. Aus diesem Grund ist die effektive Adsorptionsfläche des Rußes und somit die Fähigkeit Radikale an der Oberfläche zu binden, erhöht worden. Die effektive Radikalkonzentration wird somit in der Anfangsphase der Polymerisation stärker heruntergesetzt, wodurch die Umsätze kleiner ausfallen als für Polymerisationen unter Luftsauerstoff. Die Differenzen der Umsätze unter Argonatmosphäre und Luftsauerstoff werden bei höheren Initiatorkonzentrationen kleiner. Auch dieser Effekt lässt sich mit der Vorstellung des erhöhten Adsorptionsvermögens der entgasten Rußpartikel erklären. Die Konzentration an Rußpartikeln blieb unverändert, wobei sich die Initiatorkonzentration verdoppelt hat. Es zerfallen in der Anfangsphase der Polymerisation zweimal so viele Initiatormoleküle zu Radikalen. Aufgrund dieser erhöhten Radikalkonzentration wird die Rußoberfläche schneller „abgesättigt“, wodurch der Einfluss der Rußpartikel in der zeitlichen Betrachtung des Gesamtsystems geringer wird. Somit sind auch die kleineren Unterschiede in den Umsätzen bei höheren Initiatorkonzentrationen zu erklären.

\subsubsection{Polymerisationen unter Stickstoffatmosphäre}

In diesem Kapitel werden konventionelle radikalische Polymerisationen unter einer Stickstoffatmosphäre durchgeführt, um auch hier den Einfluss auf die Umsätze der konventionellen radikalischen Polymerisation in Gegenwart von Ruß zu untersuchen. Die Polymerisation wird nach Variante 2 durchgeführt, wobei jede Probe zuvor für mindestens 15 Minuten mit Stickstoff durchspült worden ist. Als Referenz werden Polymerisationen mit identischer Zusammensetzung unter Luftsauerstoff herangezogen. Auf eine Abtrennung der Rußpartikel wird wie in Kapitel 8.7.2 verzichtet, da Restmengen an Polymer im Filter zurückbleiben können und somit die Umsatzbestimmung verzerrt wird. Bei den Polymerisationen unter Argonatmosphäre hat sich gezeigt, dass die Umsätze kleiner ausfallen, wenn man diese mit den Ergebnissen der Polymerisation unter Luftsauerstoff vergleicht. Das Durchspülen mit technischem Stickstoff sollte den Sauerstoff, welcher in der Polymerisationslösung adsorbiert ist, verdrängen. Die effektive Adsorptionsfläche der Rußpartikel wird somit nicht vergrößert und der Einfluss des Sauerstoffs in der Polymerisation verringert. Es werden also keine Initiatorradikale benötigt, um die Biradikale zu kompensieren. Die Polymerisationen unter 
Stickstoffatmosphäre müssten demnach höhere Umsätze erreichen als unter Luftsauerstoff. In Tabelle 8.7.3 ist die Zusammensetzung des Polymerisationsansatzes wiedergegeben. Die Rußmenge ist so gewählt, dass bei einer RAFT-Polymerisation von der Oberfläche eine Beladungsdichte von $\rho_{\text {Agens }}=0,192 \mathrm{mmol} / \mathrm{g}$ erreicht wird. Hierbei wird davon ausgegangen, dass die RAFT-Agens-Konzentration gleich der Initiatorkonzentration ist. Diese Beladungsdichte ist somit vergleichbar mit der modifizierten Rußprobe „FABPTTCP02“ (vgl. Tabelle 8.6.5). Es werden nach unterschiedlichen Reaktionszeiten Proben entnommen.

Tabelle 8.7.3

Typische Zusammensetzung des Polymerisationsansatzes der konventionellen radikalischen Polymerisation unter Luftsauerstoff bzw. unter Stickstoffatmosphäre:

\begin{tabular}{|c|c|c|}
\hline Initiator & ACC & $0,040 \mathrm{Mol} \%$ \\
\hline Monomer & Styrol & $42,6 \mathrm{Mol} \%$ \\
\hline Lösungsmittel & Toluol & $42,6 \mathrm{Mol} \%$ \\
\hline Feststoff & Ruß & $14,8 \mathrm{Mol} \%$ \\
\hline
\end{tabular}

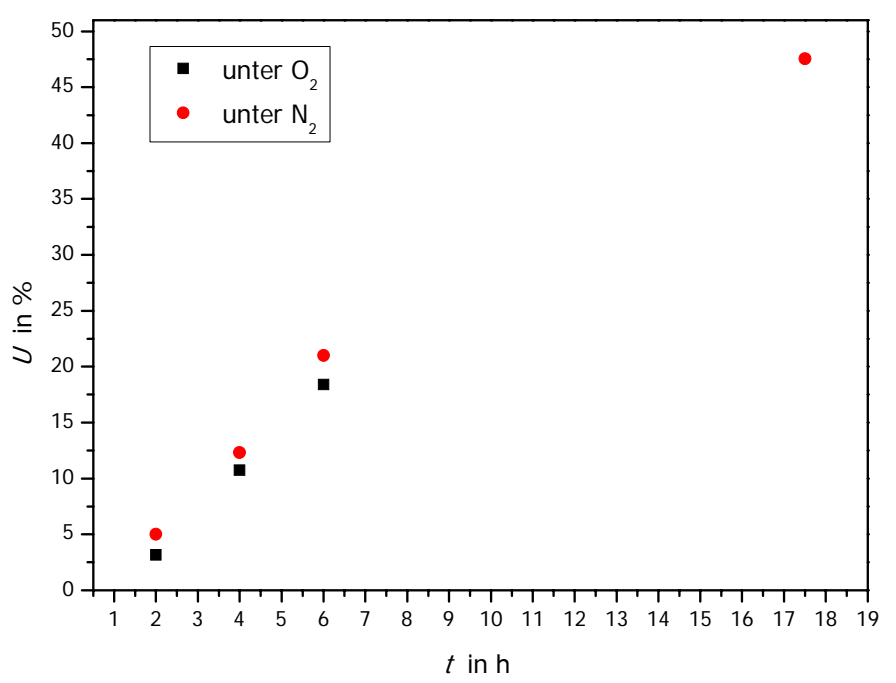

\section{Abbildung 8.7.6}

Darstellung der Umsatz-Zeit-Verläufe für konventionelle radikalische Polymerisationen in Gegenwart von Ruß unter Luftsauerstoff bzw. unter Stickstoffatmosphäre. 


\section{RAFT-Polymerisation an Industrierußen}

In Abbildung 8.7.6 ist der Umsatz-Zeit-Verlauf für die unterschiedlichen Polymerisationen aufgetragen. Wie in den theoretischen Überlegungen vermutet, erreichen die Polymerisationen unter Stickstoffatmosphäre höhere Umsätze. Es ist zu erkennen, dass die Steigungen der Umsatzverläufe ähnlich sind. Dieser Effekt wurde bereits bei den konventionellen radikalischen Polymerisationen in Gegenwart von Ruß beobachtet (Kapitel 8.7.1), wobei bei diesen Experimenten die Rußkonzentration variiert wurden. Die Radikalkonzentration wird also am Anfang der Polymerisation reduziert. In diesem Fall liegt die Ursache allerdings nicht an den verschiedenen Rußanteilen, sondern ist in der unterschiedlichen Präparation der Proben zu suchen.

\subsection{Polymerisationen in Gegenwart der Ankergruppe „FTBP“}

In den vorherigen Kapiteln ist der Einfluss von Industrieruß auf die konventionelle radikalische Polymerisation unter verschiedenen Reaktionsbedingungen untersucht worden. In diesem Kapitel wird nun die Ankergruppe des RAFT-Agens, welche ein Thioesterfunktionalität besitzt (Abbildung 8.8.1), in der konventionellen radikalischen Polymerisation betrachtet.

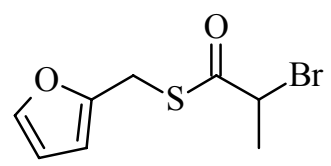

\section{Abbildung 8.8.1}

Strukturformel der Ankergruppe „FTBP“, welche einer konventionellen radikalischen Polymerisation von Styrol in Toluol in unterschiedlichen Konzentrationen zugesetzt wird.

In diesen Experimenten soll sichergestellt werden, dass die Ankergruppe keinen Einfluss auf den Verlauf einer konventionellen radikalischen Polymerisation hat. Falls es zu radikalischen Reaktionen an der Thioesterfunktionalität kommt, könnten sich Charakteristika einer kontrollierten radikalischen Polymerisation zeigen. Ein Anstieg der mittleren Molekulargewichtsverteilungen mit dem Umsatz oder eine Polydispersität $P D I<1,5$ würden diese Vermutung verstärken. Die Ankergruppe „FTBP“ besitzt neben der Thioesterfunktionalität noch ein sekundäres Bromatom. Halogenverbindungen können Kettenübertragungsreaktionen verursachen, wodurch das kinetische Schema der konventionellen radikalischen Polymerisation erweitert wird. Falls die Ankergruppe 
„FTBP“ als Transferagens fungiert, müsste sich das mittlere Molekulargewicht mit zunehmender Konzentration der Ankergruppe verkleinern. In Tabelle 8.8.1 ist ein typischer Polymerisationsansatz zu sehen. Die Polymerisation wird nach Variante 2 durchgeführt.

Tabelle 8.8.1

Typische Zusammensetzung des Polymerisationsansatzes der konventionellen radikalischen Polymerisation in Gegenwart der Ankergruppe „FTBP“: Die Konzentration der Ankergruppe beträgt in diesem Fall $c_{0}=0,100$ Mol\% und wird im Verlauf der Untersuchungen variiert:

\begin{tabular}{|c|c|c|}
\hline „RAFT-Agens“ & FTBP & $0,100 \mathrm{Mol} \%$ \\
\hline Initiator & ACC & $0,100 \mathrm{Mol} \%$ \\
\hline Monomer & Styrol & $32,2 \mathrm{Mol} \%$ \\
\hline Lösungsmittel & Toluol & $66,6 \mathrm{Mol} \%$ \\
\hline
\end{tabular}

Die Polymerisationen werden unter Luftsauerstoff durchgeführt. Es wird auf die Zugabe von Ruß verzichtet, um das System nicht unnötig zu verkomplizieren. Als Referenz wird eine konventionelle radikalische Polymerisation ohne Ankergruppe verwendet. Die Konzentration an „FTBP“ wird von $c_{0}=0,100 \mathrm{Mol} \%$ über $c_{0}=0,500 \mathrm{Mol} \%$ auf $c_{0}=1,000 \mathrm{Mol} \%$ erhöht, wobei sich die Angaben der Zusammensetzung immer auf das Gesamtsystem beziehen. Aus diesem Grund wird die Monomerkonzentration geringfügig verringert, wenn sich die Konzentration der Ankergruppe erhöht. Es ist also zu erwarten, dass die Umsätze etwas kleiner ausfallen können. In der Auswertung der Experimente wird ein theoretisches mittleres Molekulargewicht berechnet. Diese Berechnungen gehen davon aus, dass die Ankergruppe in der gesamten Anzahl als RAFT-Agens fungiert. In Abbildung 8.8.2 sind die mittleren Molekulargewichte bzw. die Polydispersität gegen den Umsatz dargestellt. Es ist gut zu erkennen, dass die mittleren Molekulargewichte nicht mit dem Umsatz ansteigen. Der Verlauf ist mit dem einer konventionellen radikalischen Polymerisation vergleichbar. Die mittleren Molekulargewichte der Referenzproben und die experimentellen Ergebnisse sind nahezu identisch. Diese Tatsache lässt vermuten, dass die Ankergruppe „FTBP“ als schlechtes Transferagens fungiert. Die Polymerisationsgeschwindigkeit ist wie erwartet nicht reduziert worden. 


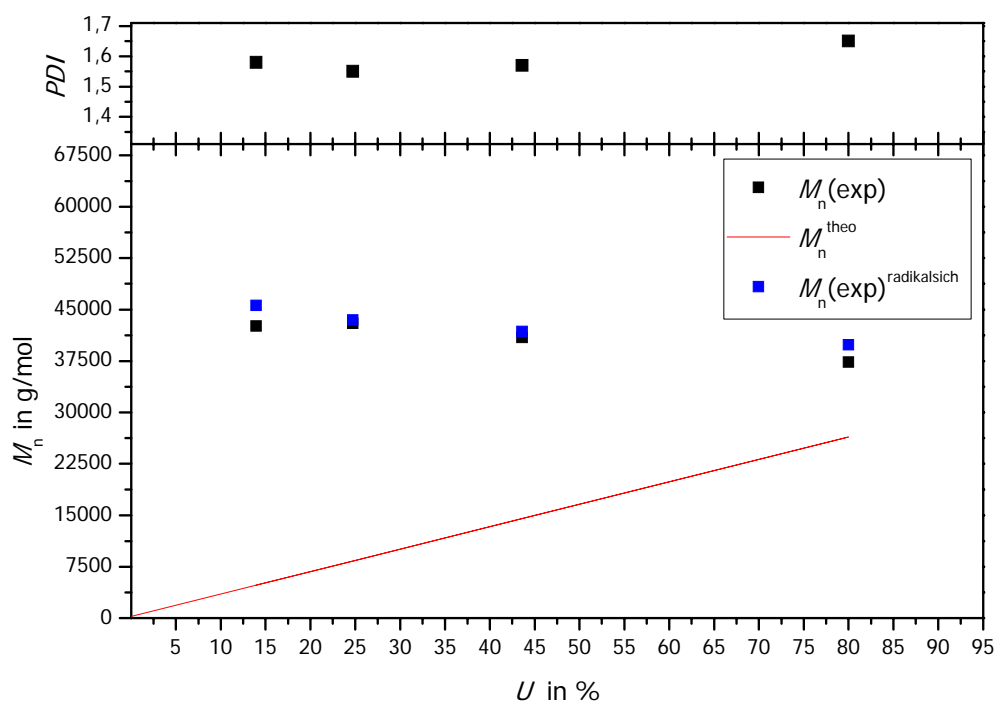

\section{Abbildung 8.8.2}

Darstellung der mittleren Molekulargewichte und der Polydispersität gegen den Umsatz: Die Initiatorkonzentration beträgt $c_{0}(A C C)=0,100 \mathrm{Mol} \%$ und die Konzentration der Ankergruppe beträgt $c_{0}(F T B P)=0,100$ Mol\%. Die rote Linie beschreibt die theoretischen mittleren Molekulargewichte unter der Annahme, dass die Ankergruppe vollständig als RAFT-Agens fungiert. Die blauen Punkte stellen die experimentellen Ergebnisse einer radikalischen Polymerisation mit gleicher Initiatorkonzentration dar.

Die Abweichungen zwischen den experimentellen Molekulargewichten und der theoretischen Voraussage sind sehr groß. Es kann also davon ausgegangen werden, dass die Thioesterfunktionalität nicht in den Mechanismus der konventionellen radikalischen Polymerisation eingreift. Die Polydispersität der einzelnen Proben liegt immer über dem Wert von $P D I=1,5$ und ist für Polystyrol, welches durch eine konventionelle radikalische Polymerisation hergestellt wurde, charakteristisch. 


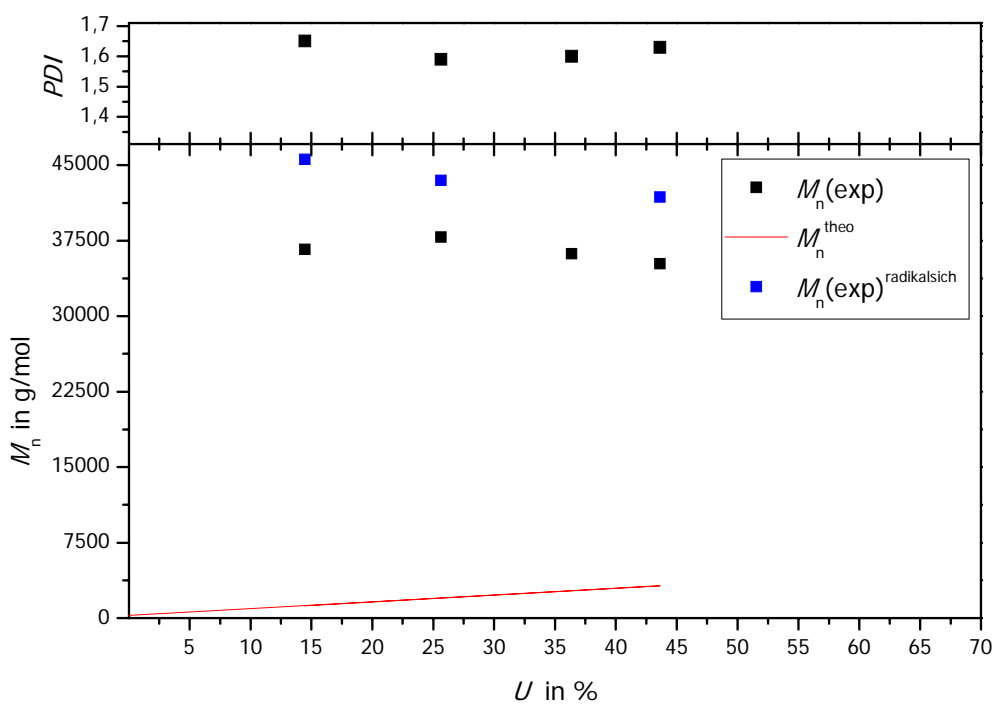

\section{Abbildung 8.8.3}

Darstellung der mittleren Molekulargewichte und der Polydispersität gegen den Umsatz: Die Initiatorkonzentration beträgt $c_{0}(A C C)=0,100 \mathrm{Mol} \%$ und die Konzentration der Ankergruppe beträgt $c_{0}(F T B P)=0,500$ Mol\%. Die rote Linie beschreibt die theoretischen mittleren Molekulargewichte unter der Annahme, dass die Ankergruppe vollständig als RAFT-Agens fungiert. Die blauen Punkte stellen die experimentellen Ergebnisse einer radikalischen Polymerisation mit gleicher Initiatorkonzentration dar.

In Abbildung 8.8.3 wurde der Anteil der Ankergruppe im Vergleich zu Abbildung 8.8.2 um den Faktor fünf erhöht. Es ist gut zu erkennen, dass sich auch in diesem Fall die Umsätze nicht verändern und somit die Polymerisationsgeschwindigkeit gleich bleibt. Auch die Polydispersitäten sind mit den Referenzproben vergleichbar und zeigen keinen Verlauf, der auf eine kontrollierte Polymerisation schließen lässt. Es ist allerdings auffällig, dass die mittleren Molekulargewichte abgenommen haben. Anscheinend treten mit höherer Konzentration der Ankergruppe vermehrt Transferreaktionen auf. Diese Tatsache wurde bereits in den Vorüberlegungen betrachtet und wirkt somit wenig überraschend. Auch in Abbildung 8.8.4 werden die erwarteten Ergebnisse bestätigt. Eine nochmalige Konzentrationserhöhung der Ankergruppe führt wiederum $\mathrm{zu}$ einer Verringerung der mittleren Molekulargewichte. Der Einfluss der Transferreaktionen nimmt nochmals zu. 


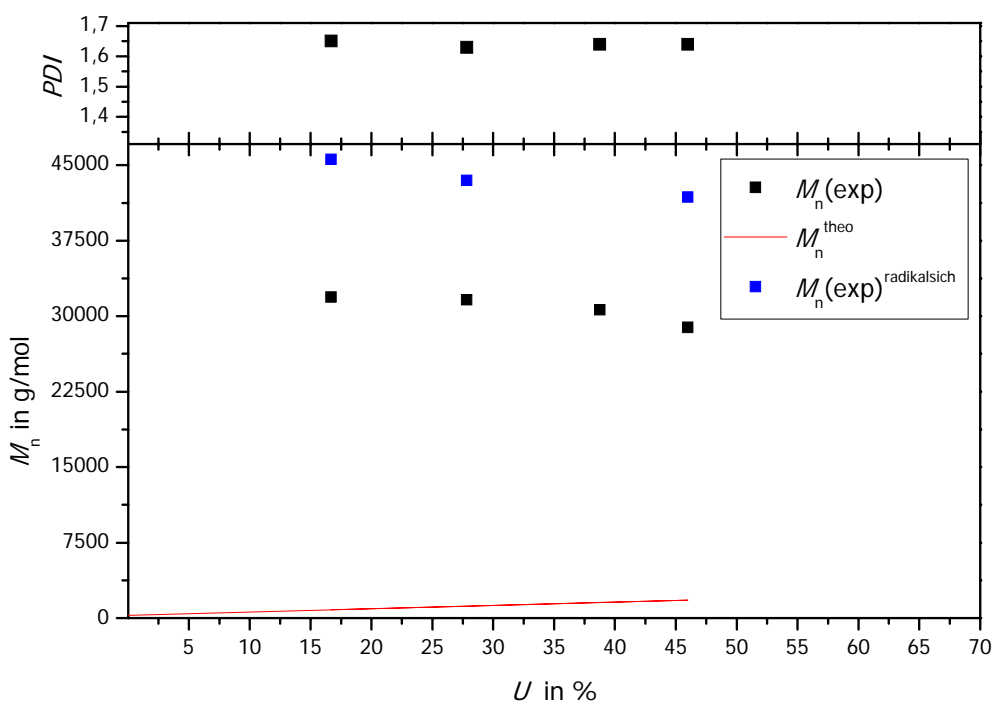

\section{Abbildung 8.8.4}

Darstellung der mittleren Molekulargewichte und der Polydispersität gegen den Umsatz: Die Initiatorkonzentration beträgt $c_{0}(A C C)=0,100 \mathrm{Mol} \%$ und die Konzentration der Ankergruppe beträgt $c_{0}(F T B P)=1,000$ Mol\%. Die rote Linie beschreibt die theoretischen mittleren Molekulargewichte unter der Annahme, dass die Ankergruppe vollständig als RAFT-Agens fungiert. Die blauen Punkte stellen die experimentellen Ergebnisse einer radikalischen Polymerisation mit gleicher Initiatorkonzentration dar.

Die Polydispersitäten steigen bei höheren Konzentrationen der Ankergruppe leicht an. Diese Tatsache ist $\mathrm{zu}$ erwarten, wenn vermehrt Transferreaktionen während der Polymerisation ablaufen. Die theoretischen Molekulargewichte $\left(M_{\mathrm{n}}^{\text {theo }}\right)$ werden immer kleiner berechnet, da die RAFT-Agens-Konzentration bei der Berechnung im Nenner steht. In Abbildung 8.8.3 und 8.8.4 werden die Konzentrationen an „FTBP“ stark angehoben, wodurch sich sehr kleine Werte in der Berechnung ergeben. Da die experimentellen Molekulargewichte nur durch die verstärkt auftretenden Übertragungsreaktionen verkleinert werden, wird Abweichung der experimentellen und der theoretischen Molekulargewichte immer größer. In der Zusammenfassung ist anzumerken, dass die Ankergruppe gut geeignet ist, um das RAFT-Agens für eine Polymerisation von der Rußoberfläche an diese anzubinden. Die Thioesterfunktionalität hat keinen Einfluss auf den Verlauf der konventionellen radikalischen Polymerisation und kann somit uneingeschränkt verwendet werden. Die Tatsache, dass die hier untersuchte Ankergruppe in höheren Konzentrationen vermehrt Transferreaktionen ermöglicht, fällt 
nicht weiter ins Gewicht, da das Bromatom in der Zielstruktur des RAFT-Agens nicht mehr vorhanden ist. An dieser Stelle im Molekül wird dann das Trithiocarbonat angebunden (Kapitel 6.2.8), um eine Kontrolle der Polymerisation zu erlauben.

\subsection{RAFT-Polymerisationen mit ungebundenem „FTBTTCP“}

In diesem Abschnitt wird die Polymerisationskontrolle des RAFT-Agens untersucht, welches später an die Rußoberfläche gebunden wird. Nachdem im vorherigen Kapitel die Ankergruppe in der radikalischen Polymerisation untersucht worden ist, werden jetzt RAFT-Polymerisationen mit Styrol durchgeführt, um die Kontrolle des RAFT-Agens zu untersuchen. Die Konzentration an Initiator und an „FTBPTTCP“ wird hierbei in verschiedenen Größenordnungen variiert, wobei das Verhältnis mit 1:1 immer gleich bleibt. Tabelle 8.9.1 zeigt eine typische Zusammensetzung des Polymerisationsansatzes. Die Polymerisation wird nach Variante 2 durchgeführt. Nach verschiedenen Reaktionszeiten werden Proben entnommen und der Umsatz gravimetrisch bestimmt. Auf eine Zugabe von Ruß wird verzichtet, um Störeinflüsse zu vermeiden und die Kontrolle des RAFT-Agens quantifizieren zu können.

\section{Tabelle 8.9.1}

Typische Zusammensetzung des Polymerisationsansatzes der RAFT-Polymerisation mit „FTBPTTCP“: Die Konzentration des RAFT-Agens beträgt in diesem Fall $c_{0}=0,100$ Mol\% und wird im Verlauf der Untersuchungen variiert.

\begin{tabular}{|c|c|c|}
\hline RAFT-Agens & FTBPTTCP & $0,100 \mathrm{Mol} \%$ \\
\hline Initiator & ACC & $0,100 \mathrm{Mol} \%$ \\
\hline Monomer & Styrol & $32,2 \mathrm{Mol} \%$ \\
\hline Lösungsmittel & Toluol & $66,6 \mathrm{Mol} \%$ \\
\hline
\end{tabular}

In Abbildung 8.9.1 sind die Ergebnisse der Polymerisation mit einer RAFT-AgensKonzentration von $c_{0}($ RAFT $)=0,100 \mathrm{Mol} \%$ wiedergegeben. Um eine Aussage über die Güte der Polymerisationskontrolle treffen zu können, werden die experimentellen Ergebnisse mit den theoretischen Molekulargewichten $M_{\mathrm{n}}^{\text {theo }}$ und $M_{\mathrm{n}}^{\text {theo }}(\mathrm{T})$ verglichen. Es ist gut zu erkennen, dass die experimentell bestimmten Molekulargewichte sehr gut den Verlauf der theoretischen Vorraussagen beschreiben. Es ist ein stetiger Anstieg der Molekulargewichte mit dem Umsatz zu verzeichnen. Die experimentellen Werte sind kleiner als der im Idealfall beschriebene Wert $M_{\mathrm{n}}^{\text {theo }}$. 


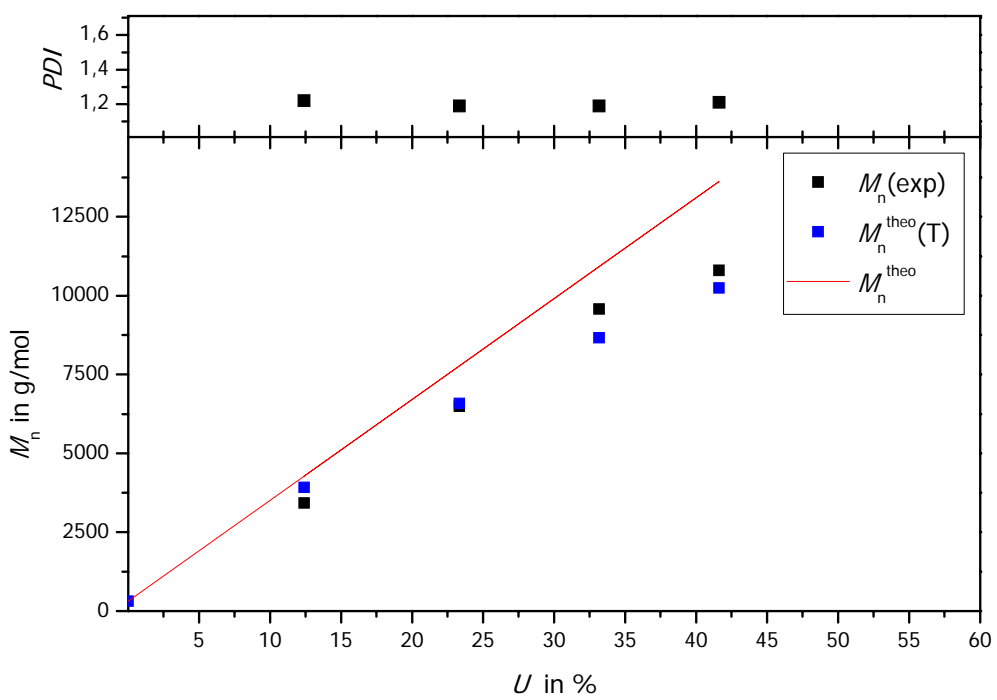

\section{Abbildung 8.9.1}

Darstellung der mittleren Molekulargewichte und der Polydispersität gegen den Umsatz: Die Initiatorkonzentration beträgt $c_{0}(A C C)==0,100 \mathrm{Mol} \%$ und die Konzentration des RAFT-Agens beträgt $c_{0}($ FTBPTTCP $)=0,100 \mathrm{Mol} \%$. Die rote Linie beschreibt die idealen, theoretischen mittleren Molekulargewichte. Die blauen Punkte stellen die theoretischen Molekulargewichte unter Berücksichtigung der Terminierung dar.

Die Polydispersitäten sind aller kleiner als $P D I=1,5$ und schwanken um einen Wert bei $P D I=1,2$. Das RAFT-Agens zeigt eine sehr gute Reaktionskontrolle und scheint für die Polymerisation mit Styrol und 1,3-Butadien bestens geeignet zu sein. Die sekundäre Abgangsgruppe ermöglicht eine schnelle Reinitiierung nachdem das Vorgleichgewicht des RAFT-Mechanismus durchlaufen wurden. Für die weiteren Untersuchungen wird die RAFT-Agens-Konzentration reduziert. Es soll bestätigt werden, dass auch bei kleinen Konzentrationen die RAFT-Polymerisation eine gute Reaktionskontrolle zeigt. In Hinblick auf die RAFT-Polymerisation von der Rußoberfläche erscheint dies sinnvoll, da aufgrund der Beladungsdichten der Rußpartikel keine höheren Konzentrationen an kontrollierenden Agens vorliegen.

In Abbildung 8.9.2 ist der Verlauf der experimentellen Ergebnisse der Polymerisation mit einer Konzentration des RAFT-Agens von $c_{0}=0,040 \mathrm{Mol}$ erkennbar. Da die Initiatorkonzentration ebenfalls angepasst wurde, ergeben sich kleinere Umsätze nach identischen Polymerisationszeiten. 


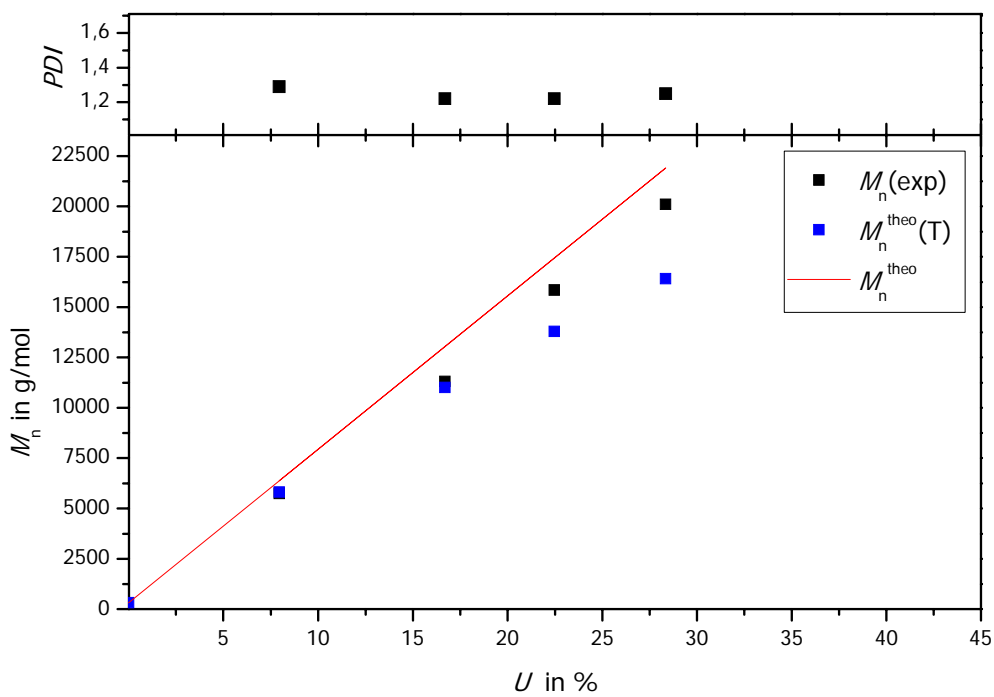

\section{Abbildung 8.9.2}

Darstellung der mittleren Molekulargewichte und der Polydispersität gegen den Umsatz: Die Initiatorkonzentration beträgt $c_{0}(A C C)=0,040 \mathrm{Mol} \%$ und die Konzentration des RAFT-Agens beträgt $c_{0}(F T B P T T C P)=0,040 \mathrm{Mol} \%$. Die rote Linie beschreibt die idealen, theoretischen mittleren Molekulargewichte. Die blauen Punkte stellen die theoretischen Molekulargewichte unter Berücksichtigung der Terminierung dar.

Abbildung 8.9.2 beschreibt ebenfalls eine sehr gute Polymerisationskontrolle. Die experimentellen Molekulargewichte steigen nahezu linear mit dem Umsatz an. Auch die theoretischen Vorraussagen werden in ihrem Verlauf von den experimentellen Ergebnissen beschrieben. Die besten Übereinstimmungen werden mit den Berechnungen von $M_{\mathrm{n}}^{\text {theo }}(\mathrm{T})$ erzielt. Dieser Umstand ist nicht weiter verwunderlich, da hier die realistischste Beschreibung der Kinetik der RAFT-Polymerisation dargestellt wird.

Die Polydispersitäten sind etwas höher als im Vergleich zu den Resultaten in Abbildung 8.9.1. Sie schwanken in einem Bereich von $P D I=1,2-1,3$ und deuten ebenfalls auf eine gute Polymerisationskontrolle hin. In Abbildung 8.9.3 werden die Ergebnisse der RAFTPolymerisation mit einer Konzentration an RAFT-Agens von $c_{0}=0,020 \mathrm{Mol} \%$ dargestellt. In diesem Fall sollte sich die Reaktionskontrolle etwas verschlechtern, da weniger kontrollierendes Agens zur Verfügung steht. Die Initiatorkonzentration ist um den gleichen Faktor verringert worden, wodurch wiederum kleinere Umsätze nach gleichen Reaktionszeiten erzielt werden. 


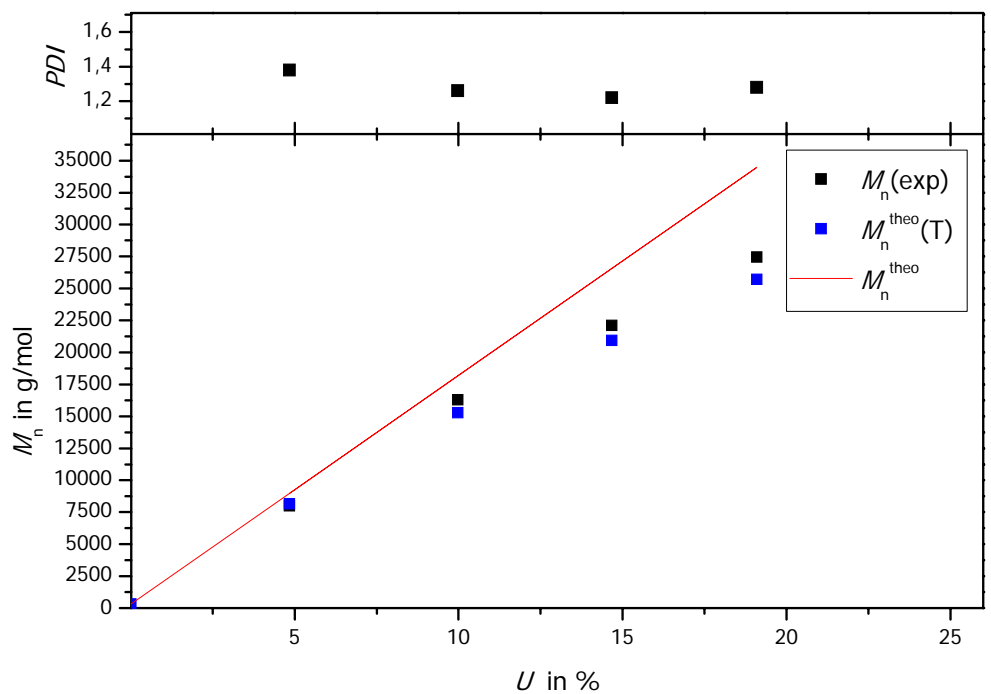

\section{Abbildung 8.9.3}

Darstellung der mittleren Molekulargewichte und der Polydispersität gegen den Umsatz: Die Initiatorkonzentration beträgt $c_{0}(A C C)=0,020 \mathrm{Mol} \%$ und die Konzentration des RAFT-Agens beträgt $c_{0}(F T B P T T C P)=0,020 \mathrm{Mol} \%$. Die rote Linie beschreibt die idealen, theoretischen mittleren Molekulargewichte. Die blauen Punkte stellen die theoretischen Molekulargewichte unter Berücksichtigung der Terminierung dar.

Die Entwicklung der experimentellen Molekulargewichte mit dem Umsatz spiegelt (Abbildung 8.9.3) eine sehr gute Polymerisationskontrolle wider. Der Anstieg der Molekulargewichte mit dem Umsatz ist stetig und verläuft nahezu linear. Es kommt zu einer sehr guten Deckung mit den theoretischen Vorraussagen nach $M_{\mathrm{n}}{ }^{\text {theo }}(\mathrm{T})$. Auch die Werte der Polydispersität liegen unterhalb von $P D I=1,5 . \mathrm{Im}$ direkten Vergleich sind die Absolutwerte der Polydispersität etwas größer, als in den Polymerisationen mit höheren RAFT-Agens-Konzentrationen.

Die Untersuchungen zeigen, dass FTBPTTCP hervorragend geeignet ist, um die RAFTPolymerisation von Styrol bzw. 1,3-Butadien zu kontrollieren. Auch die beobachteten Effekte der Ankergruppe FTBP, welche in höheren Konzentrationen Übertragungsreaktionen ermöglicht, werden vom RAFT-Agens nicht bestätigt. Dies ist nicht weiter verwunderlich, da in der Struktur des RAFT-Agens das Halogen fehlt, welches als Ursache für Transferreaktionen angesehen wird. 


\subsection{RAFT-Polymerisationen in Gegenwart von Ruß}

Es hat sich gezeigt, dass die konventionelle radikalische Polymerisation in Gegenwart von Ruß unter Umsatzeinbußen problemlos funktioniert. Die Untersuchungen ergeben, dass eine höhere Konzentration an Rußpartikeln die Umsätze der einzelnen Proben weiter nach unten korrigiert. In diesem Kapitel werden RAFT-Polymerisationen mit Industrierußen durchgeführt. Hierzu wird in Kapitel 8.10.1 im Rahmen von Vorversuchen das bereits erprobtes RAFT-Agens Bis(1-phenylethyl)trithiocarbonat (BPETC) hinzugegeben. Es soll getestet werden, ob der Ruß einen Einfluss auf den Mechanismus der RAFTPolymerisation hat. Im Kapitel 8.10.2 wird das kontrollierende Agens untersucht, welches auch in der RAFT-Polymerisation von der Oberfläche verwendet wird.

\subsubsection{RAFT-Polymerisationen mit „BPETC“}

In diesem Abschnitt werden Lösungsmittelpolymerisationen von Styrol in Toluol mit BPETC als RAFT-Agens durchgeführt. Als Referenz wird eine Polymerisation ohne Zusatz von Ruß dienen. Bei den anderen Polymerisationsansätzen wird die Rußmenge kontinuierlich von 2,5 Mol\% über 20,4 Mol\% bis zu 41,4 Mol\% bezogen auf das Gesamtsystem erhöht. Die Konzentrationen an RAFT-Agens und Initiator sind gleich und betragen $c_{0}=0,100 \mathrm{Mol} \%$. Für die experimentelle Reihe werden vier identische Lösungen aus RAFT-Agens, Lösungsmittel, Monomer und Initiator angesetzt, welche dann mit unterschiedlichen Anteilen an Ruß versetzt werden. Die Polymerisationen werden nach Variante 1 durchgeführt. In Tabelle 8.10.1 ist ein typischer Polymerisationsansatz aufgeführt.

Tabelle 8.10.1

Typische Zusammensetzung des Polymerisationsansatzes der RAFT-Polymerisation mit „BPETC“ in Gegenwart von Ruß: Die Konzentration der Rußpartikel beträgt in diesem Fall 2,5 Mol \% und wird im Verlauf der Untersuchungen variiert.

\begin{tabular}{|c|c|c|}
\hline RAFT-Agens & BPETC & $0,100 \mathrm{Mol} \%$ \\
\hline Initiator & ACC & $0,100 \mathrm{Mol} \%$ \\
\hline Monomer & Styrol & $32,4 \mathrm{Mol} \%$ \\
\hline Lösungsmittel & Toluol & $64,9 \mathrm{Mol} \%$ \\
\hline Füllstoff & Ruß & $2,5 \mathrm{Mol} \%$ \\
\hline
\end{tabular}


Die Polymerisationsgeschwindigkeit sollte sich bei größeren Anteilen an Ruß verkleinern, da die effektive Initiatorkonzentration verringert wird (vgl. Kapitel 8.7.1). Es müssten also kleinere Umsätze bestimmt werden, wenn die Konzentration an Ruß erhöht wird. Tabelle 8.10.2 zeigt die Ergebnisse.

Tabelle 8.10.2

Auflistung der Ergebnisse der RAFT-Polymerisation von Styrol mit „BPETC“ in Gegenwart von variierenden Anteilen an Ruß:

\begin{tabular}{|c|c|c|c|c|c|}
\hline \multirow{3}{*}{$t$ in min } & $\begin{array}{c}c_{0}(\mathrm{Ru} \beta) \text { in } \\
\text { Mol\% }\end{array}$ & $U$ in $\%$ & $M_{\mathrm{n}}$ in $\mathrm{g} / \mathrm{mol}$ & $P D I$ & $\begin{array}{c}M_{\mathrm{n}}^{\text {theo }} \\
\mathrm{g} / \mathrm{mol}\end{array}$ \\
\hline \multirow{3}{*}{210} & 0,0 & 19,42 & 6589 & 1,14 & 7091 \\
\cline { 2 - 6 } & 2,5 & 17,50 & 6664 & 1,15 & 6394 \\
\cline { 2 - 6 } & 20,4 & 15,17 & 7961 & 1,16 & 5546 \\
\cline { 2 - 6 } & 41,4 & 14,07 & 6869 & 1,18 & 5133 \\
\hline \multirow{3}{*}{420} & 0,0 & 35,93 & 11409 & 1,14 & 12850 \\
\cline { 2 - 6 } & 2,5 & 32,09 & 11449 & 1,15 & 11438 \\
\cline { 2 - 6 } & 20,4 & 29,42 & 13684 & 1,14 & 10457 \\
\cline { 2 - 6 } & 41,4 & 26,84 & 13071 & 1,13 & 9505 \\
\hline
\end{tabular}

Die Ergebnisse in Tabelle 8.10.2 entsprechen den theoretischen Vorüberlegungen und spiegeln ebenfalls die Untersuchungen der konventionellen radikalischen Polymerisation wider. Es ist gut zu erkennen, dass die Umsätze der Proben mit zunehmender Rußkonzentration kleiner werden. An dieser Stelle ist allerdings anzumerken, dass die Verhältnismäßigkeit zwischen Abnahme des Umsatzes und der Rußoberfläche nicht gewahrt ist. Die Polymerisationsgeschwindigkeit verringert sich, da Radikale an die Oberfläche des Rußes gebunden werden und somit nicht mehr dem kinetischen Schema zur Verfügung stehen. Nach diesen Überlegungen müsste also die Umsatzabnahme proportional zur Rußoberfläche bzw. der Rußkonzentration sein. Dies ist allerdings nur bedingt der Fall. Die Ursache könnte hierbei in der Präparation der Proben zu suchen sein. Die Polymerisationen werden in einem Thermoblock durchgeführt, welcher durch einen Schüttler bewegt wird, um eine Durchmischung zu gewährleisten. Besonders bei hohen Anteilen an Ruß kann es jedoch sein, dass diese Bewegung nicht ausreichend ist, um ein Absetzen des Rußes zu verhindern. Somit wird die effektive Rußoberfläche, welche dem Polymerisationsgeschehen zur Verfügung steht, verkleinert. Diese Experimente wurden in 
einem sehr frühen Stadium der Untersuchungen von Polymerisationen in Gegenwart von Ruß durchgeführt. In den späteren Experimenten wurden deshalb die Reaktionen in Schraubdeckelgläsern, welche mit einem Rührer versehen sind, in einem Ölbad durchgeführt. Trotzdem bestätigen diese Ergebnisse, dass eine RAFT-Polymerisation in Gegenwart von Ruß erfolgreich und mit einer guten Kontrolle abläuft. Die Polydispersitäten schwanken um den Wert von $P D I=1,15$ und weisen eindeutig auf eine kontrollierte Polymerisation hin.

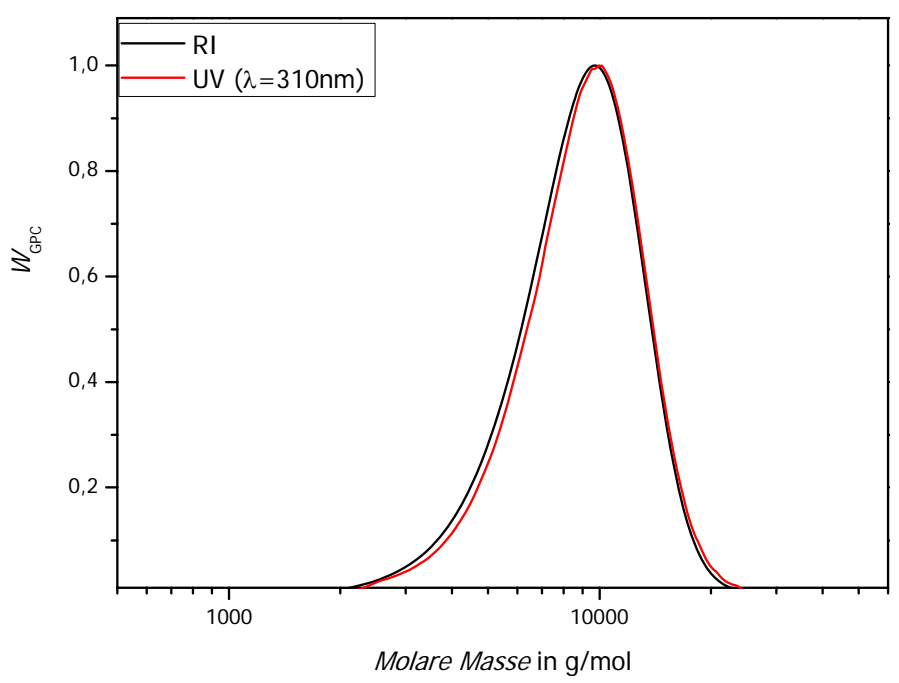

\begin{abstract}
Abbildung 8.10.1
Auftragung der normierten GPC-Detektorsignale gegen die Molmasse. Zusammensetzung: $c_{0}($ Toluol $)=53 \mathrm{Mol} \%, \quad c_{0}($ BPETC $)=c_{0}($ ACC $)=0,010 \mathrm{Mol} \%, \quad c_{0}($ Sty $)=26,6 \mathrm{Mol} \%, \quad c_{0}($ Ruß $)=20,4$ Mol\%, Auswertung: $U=15,17 \%, M_{\mathrm{n}}=7960 \mathrm{~g} / \mathrm{mol}, M_{\mathrm{n}}^{\text {theo }}=5546 \mathrm{~g} / \mathrm{mol}, P D I=1,15$.
\end{abstract}

Die experimentellen Molekulargewichte steigen mit dem Umsatz an und die theoretischen Vorraussagen der mittleren Molekulargewichte $M_{\mathrm{n}}^{\text {theo }}$ werden gut erfüllt. Auffällig ist allerdings, dass bei höheren Rußkonzentrationen die Molekulargewichte größer werden und auch den theoretischen Wert übersteigen. Dieses Phänomen lässt sich nur durch eine reduzierte effektive RAFT-Agens-Konzentration erklären. Es ist gut vorstellbar, dass die Phenylgruppen des kontrollierenden Agens eine starke Wechselwirkung mit dem ausgedehnten $\pi$-Elektronensystem des Rußes ausüben und somit an der Oberfläche physikalisch gebunden werden. Aus diesem Grund steht weniger RAFT-Agens dem Polymerisationsgeschehen zur Verfügung, wodurch die experimentellen 
Molekulargewichte größer sind als erwartet. Um einen weiteren Beweis der erfolgreichen RAFT-Polymerisation $\mathrm{zu}$ liefern, werden in Abbildung 8.10.1 die Molekulargewichtsverteilungen des Massendetektors und des UV-Detektors der GPCAnalyse verglichen (vgl. Kapitel 7.2). Die UV-Absorbanz liegt im makromolekularen Bereich und die Übereinstimmung der Molekulargewichtsverteilungen ist hervorragend.

\subsubsection{RAFT-Polymerisationen mit „FABPTTCP“}

In diesem Kapitel wird das RAFT-Agens, welches später an die Oberfläche gebunden wird, in Gegenwart von Ruß polymerisiert. Die Untersuchungen im Kapitel 8.10.1 belegen, dass der RAFT-Mechanismus nicht von den Rußpartikeln gestört wird. Lediglich die Polymerisationsgeschwindigkeit verlangsamt sich und die effektive RAFT-AgensKonzentration wird etwas verringert, wodurch die Molekulargewichte größer sind als erwartet. Es wird eine Lösungsmittelpolymerisation von Styrol in Toluol durchgeführt. Die Polymerisation wird nach Variante 1 durchgeführt. Eine Polymerisation ohne Rußzugabe dient als Referenz. In Tabelle 8.10.3 ist ein typischer Reaktionsansatz gelistet.

\section{Tabelle 8.10.3}

Exemplarische Zusammensetzung des Polymerisationsansatzes der RAFT-Polymerisation mit „FABPTTCP“ in Gegenwart von Ruß: Die Konzentration der Rußpartikel beträgt in diesem Fall 9,0 Mol\% und wird im Verlauf der Untersuchungen variiert.

\begin{tabular}{|c|c|c|}
\hline RAFT-Agens & FABPTTCP & $0,030 \mathrm{Mol} \%$ \\
\hline Initiator & ACC & $0,100 \mathrm{Mol} \%$ \\
\hline Monomer & Styrol & $30,3 \mathrm{Mol} \%$ \\
\hline Lösungsmittel & Toluol & $64,6 \mathrm{Mol} \%$ \\
\hline Füllstoff & Ruß & $9,0 \mathrm{Mol} \%$ \\
\hline
\end{tabular}




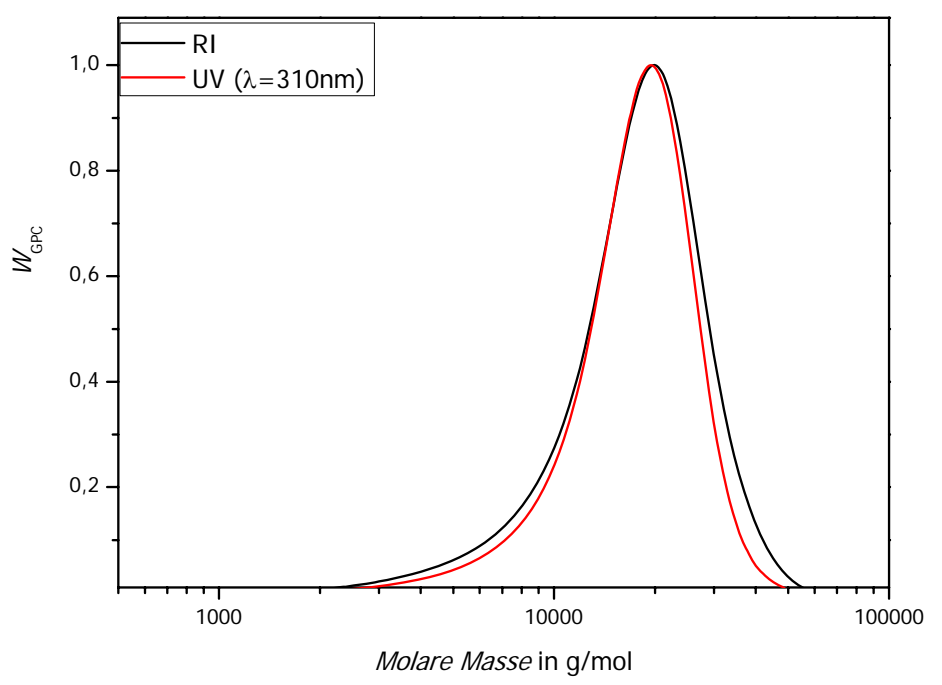

Abbildung 8.10.2

Auftragung der normierten GPC-Detektorsignale gegen die Molmasse. Zusammensetzung: $c_{o}$ (Toluol) $=64,6 \mathrm{Mol} \%, \quad c_{0}$ (FABPTTCP $)=0,030 \mathrm{Mol} \%, \quad c_{0}($ ACC $)=0,010 \mathrm{Mol} \%, \quad c_{0}($ Sty $)=30,3$ $M o l \%, c_{0}(R u ß)=9,0 M o l \%$, Auswertung: $U=10,56 \%, M_{\mathrm{n}}=14851 \mathrm{~g} / \mathrm{mol}, M_{\mathrm{n}}^{\text {theo }}=11906 \mathrm{~g} / \mathrm{mol}$, $P D I=1,29$.

In Abbildung 8.10.2 sind die Molekulargewichtsverteilungen des Massendetektors und des UV-Detektors der GPC ersichtlich. Die UV-Absorbanz liegt im makromolekularen Bereich und beide Verteilungen liegen fast aufeinander. Es kann also davon ausgegangen werden, dass jede Polymerkette eine RAFT-Gruppe trägt und somit wenig ,totes“ Material angefallen ist. Die Polydispersität dieser Verteilung beträgt $P D I=1,29$ und deutet somit auf eine gute Polymerisationskontrolle hin. Auch die Entwicklung der Molekulargewichte mit dem Umsatz (Abbildung 8.10.3) beschreibt einen lebenden Charakter der Polymerisation. Die Ergebnisse sind vergleichbar mit denen, die bei der RAFT-Polymerisation mit „BPETC“ in Gegenwart von Ruß beschrieben wurden. Bei Erhöhung der Rußkonzentration nimmt der Umsatz ab und die tatsächlichen Molekulargewichte werden größer als theoretisch angenommen. Auch in diesem Fall kann davon ausgegangen werden, dass das RAFT-Agens aufgrund von Wechselwirkungen an der Rußoberfläche adsorbiert wird. Die Polydispersitäten aller Proben liegen unterhalb von $P D I=1,5$. 


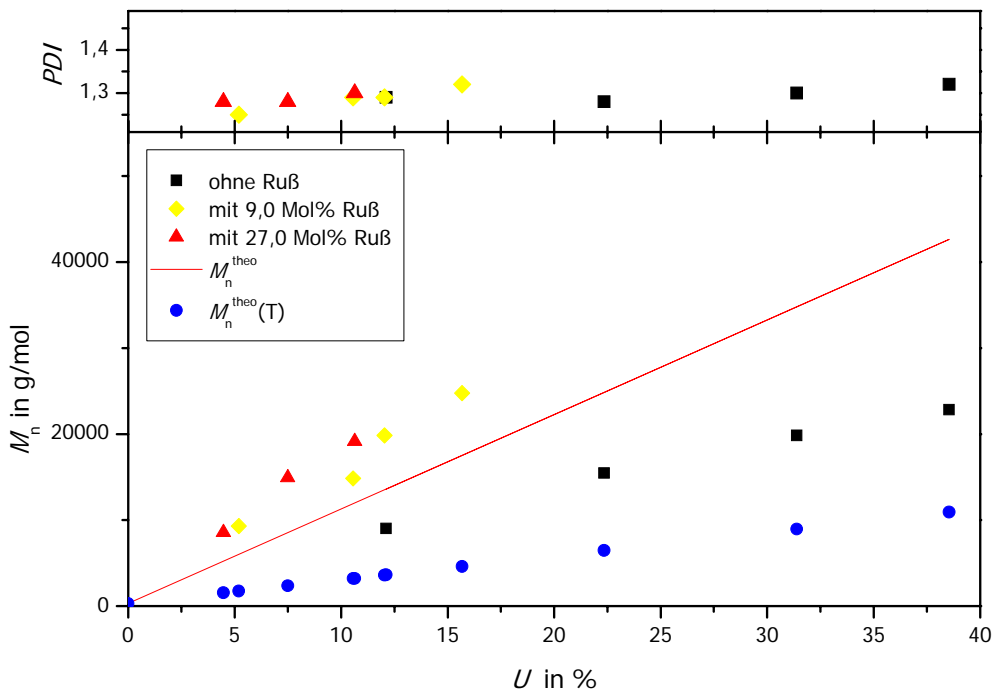

\section{Abbildung 8.10.3}

Darstellung der mittleren Molekulargewichte (PiL) und der Polydispersität gegen den Umsatz: Die Initiatorkonzentration beträgt $c_{0}(A C C)=0,100$ Mol\% und die Konzentration des RAFTAgens beträgt $c_{0}(F A B P T T C P)=0,030 \mathrm{Mol} \%$. Die rote Linie beschreibt die idealen, theoretischen mittleren Molekulargewichte. Die blauen Punkte stellen die theoretischen Molekulargewichte unter Berücksichtigung der Terminierung dar.

In der Summe gesehen sind RAFT-Polymerisationen in Gegenwart von Industrierußen möglich und erzielen ebenfalls eine sehr gute Kontrolle. Allerdings kommt es zu Wechselwirkungen zwischen dem RAFT-Agens in Lösung und der Rußoberfläche, wodurch die effektive Konzentration an kontrollierendem Agens vermindert wird. Weiterhin wurden die Ergebnisse der konventionellen radikalischen Polymerisation bestätigt. 


\subsection{RAFT-Polymerisation von der Rußoberfläche}

Nachdem alle ungeklärten Einflüsse der Rußpartikel auf den Verlauf einer konventionellen bzw. kontrollierten radikalischen Polymerisation hinreichend untersucht worden sind, werden in diesem Kapitel RAFT-Polymerisationen direkt von der Rußoberfläche durchgeführt. Die vorbehandelten Rußpartikel (Kapitel 8.6) werden in einer Lösungsmittelpolymerisation von Styrol in Toluol verwendet. Es wird kein „freies“ RAFT-Agens zugesetzt, um sicher zu gehen, dass eine Kontrolle der Polymerisation nur von den RAFT-Agenzien auf der Oberfläche hervorgerufen wird. Es ist also zu erwarten, dass die Reaktionskontrolle nicht so gut ist wie in den vorherigen Experimenten. Es wird ein stärker ausgeprägtes Hybridverhalten erwartet, da die RAFT-Agenzien an der Oberfläche nicht sofort von Makroradikalen erreicht werden. Dadurch werden die Gleichgewichte des RAFT-Mechanismus langsamer durchlaufen und mehr „totes“ Material wird während der Polymerisation gebildet. Aus diesen Überlegungen geht hervor, dass die Molekulargewichte bereits bei kleinen Umsätzen sehr stark ansteigen, da sich die Charakteristika der konventionellen radikalischen Polymerisation mit denen der kontrollierten Polymerisation kombinieren.

\subsubsection{RAFT-Polymerisation von der Oberfläche mit „FABPTTCP“}

In diesem Kapitel werden RAFT-Polymerisationen von der Rußoberfläche durchgeführt. Als kontrollierendes Agens wird „FABPTTCP“ verwendet, welches auf den Rußpartikeln mit einer Beladungsdichte von $\rho_{\text {Agens }}=0,118 \mathrm{mmol} / \mathrm{g}$ verankert wurden. Dies entspricht der Probe FABPTTCP01 (vgl. Tabelle 8.6.4). Tabelle 8.11.1 gibt die Zusammensetzung der Polymerisation wieder.

Tabelle 8.11.1

Zusammensetzung des Polymerisationsansatzes der RAFT-Polymerisation von der Rußoberfläche mit "FABPTTCP":

\begin{tabular}{|c|c|c|}
\hline RAFT-Agens & FABPTTCP & $0,020 \mathrm{Mol} \%$ \\
\hline Initiator & ACC & $0,020 \mathrm{Mol} \%$ \\
\hline Monomer & Styrol & $29,2 \mathrm{Mol} \%$ \\
\hline Lösungsmittel & Toluol & $58,3 \mathrm{Mol} \%$ \\
\hline $\begin{array}{c}\text { modifizierter } \\
\text { Füllstoff }\end{array}$ & Ruß & $12,4 \mathrm{Mol} \%$ \\
\hline
\end{tabular}


Die Polymerisation wird nach Variante 2 durchgeführt.

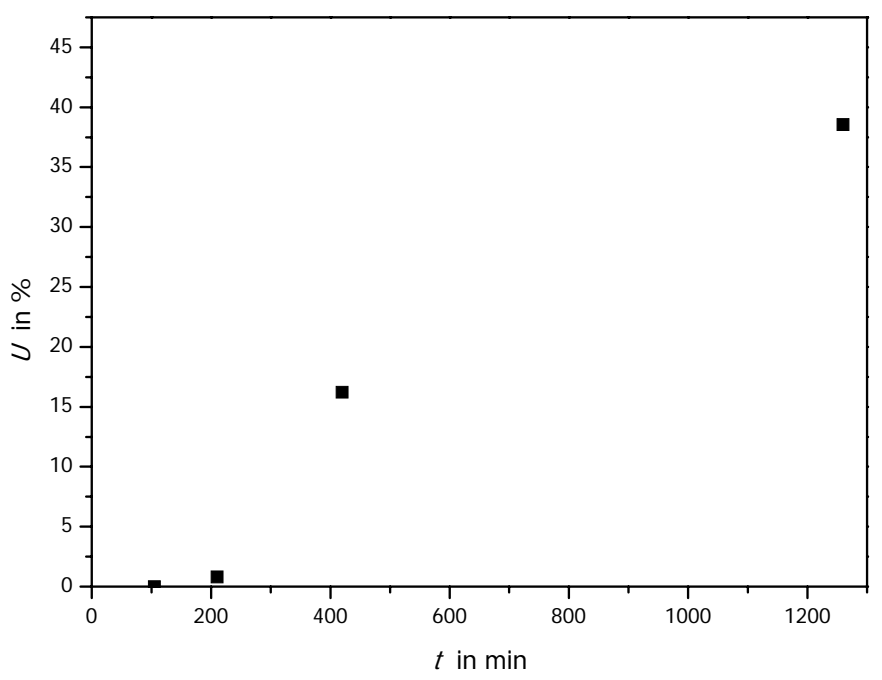

\begin{abstract}
Abbildung 8.11.1
Darstellung der Umsatz-Zeit-Verläufe des Polymers in Lösung (PiL) für eine kontrollierte radikalische RAFT-Polymerisation von der Rußoberfläche mit einer Initiatorkonzentrationen von $c_{0}(A C C)=0,020 \mathrm{Mol} \%$ und einer Rußkonzentration von $\left.c_{0}(R u \beta)\right]=12,38 \mathrm{Mol} \%$. Die Beladungsdichte auf der Rußoberfläche beträgt $\rho_{\text {Agens }}=0,118 \mathrm{mmol} / \mathrm{g}$, wodurch eine RAFTAgens-Konzentration von $c_{0}($ FABPTTCP $)=0,020$ Mol\% erreicht wird.
\end{abstract}

In Abbildung 8.11.1 ist der Verlauf der Umsatz-Zeit-Kurve abgebildet. Es ist gut zu erkennen, dass die Polymerisationsgeschwindigkeit besonders in der Anfangsphase der Polymerisation stark heruntergesetzt wird. Anscheinend muss die Rußoberfläche erstmal abgesättigt werden, bevor die Polymerisation startet. Erst nach einer Reaktionszeit von $t=200$ min steigt der Umsatz fast linear mit der Zeit an. Dieses Ergebnis wurde bereits bei der konventionellen radikalischen Polymerisation in Gegenwart von Industrierußen beobachtet (Kapitel 8.7) und bestätigt das Potenzial von Rußen Radikale auf der Oberfläche zu binden.

In Abbildung 8.11.2 ist die Entwicklung der mittleren Molekulargewichte mit dem Umsatz dargestellt. Es ist ein sehr starkes Hybridverhalten zu erkennen. Am Anfang steigt das Molekulargewicht sprunghaft an und wächst dann mit zunehmendem Umsatz. Durch den Verlauf wird der erste Hinweis geliefert, dass die RAFT-Polymerisation von der Oberfläche erfolgreich verlaufen ist. Man muss bei der Interpretation der Ergebnisse 
beachten, dass die Analyse der Molekulargewichtsverteilungen mit dem Polymermaterial erfolgt, welches aufgrund des R-Gruppen-Ansatzes in Lösung (PiL) gebildet wurde.

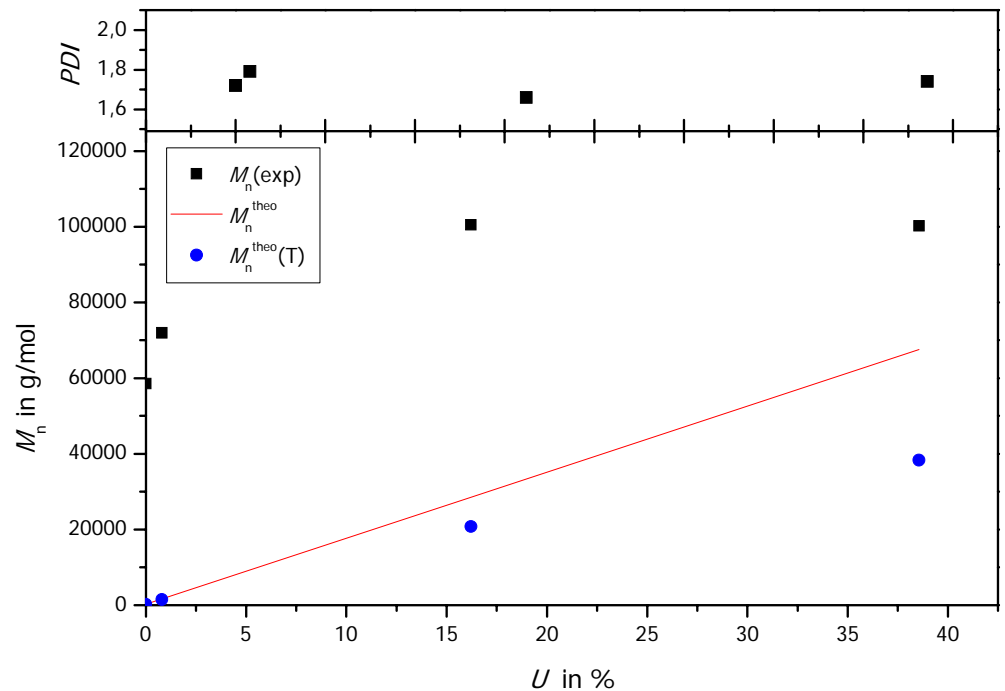

\section{Abbildung 8.11.2}

Darstellung der mittleren Molekulargewichte (PiL) und der Polydispersität gegen den Umsatz: Die Initiatorkonzentration beträgt $c_{0}(A C C)=0,020 \mathrm{Mol} \%$ und die Konzentration des RAFTAgens beträgt $c_{0}(F A B P T T C P)=0,020 \mathrm{Mol} \%$. Die rote Linie beschreibt die idealen, theoretischen mittleren Molekulargewichte. Die blauen Punkte stellen die theoretischen Molekulargewichte unter Berücksichtigung der Terminierung dar.

Die Konzentration an RAFT-Agens ist sehr gering und wird höchstwahrscheinlich noch durch die Wechselwirkungen mit der Rußoberfläche zusätzlich erniedrigt, wodurch sich die Kontrolle der Polymerisation weiter verschlechtert. Die Polydispersität der einzelnen Proben liegen in einem Bereich von $P D I=1,7$. Aufgrund des heterogenen Polymerisationsansatzes und der geringen Konzentration an RAFT-Agens, welche nur an der Oberfläche gebunden ist, sind diese Werte erstaunlich niedrig. Um einen weiteren Beweis der erfolgreichen RAFT-Polymerisation von der Oberfläche zu generieren, sind in Abbildung 8.11.3 die normierten Molekulargewichtsverteilungen des UV-Detektors und des Massensignals aufgetragen. Wie bereits in Kapitel 7.2 beschrieben, kann aus der Interpretation der Molekulargewichtsverteilungen auf die Polymerisationskontrolle geschlossen werden. Auch in diesem Fall ist eine klare Absorbanz des UV-Signals bei einer Wellenlänge von $\lambda=310 \mathrm{~nm}$ erkennbar, welche sich im makromolekularen Bereich 


\section{RAFT-Polymerisation an Industrierußen}

befindet. Das Signal-Rausch-Verhältnis des Signals des UV-Detektors ist sehr schlecht, d.h. die Detektion der RAFT-Gruppen ist aufgrund der geringen Ausgangskonzentration an RAFT-Agenzien erschwert. Es ist im Laufe der Polymerisation viel „totes“ Material entstanden, was sich auch im ausgeprägten Hybridverhalten widerspiegelt.

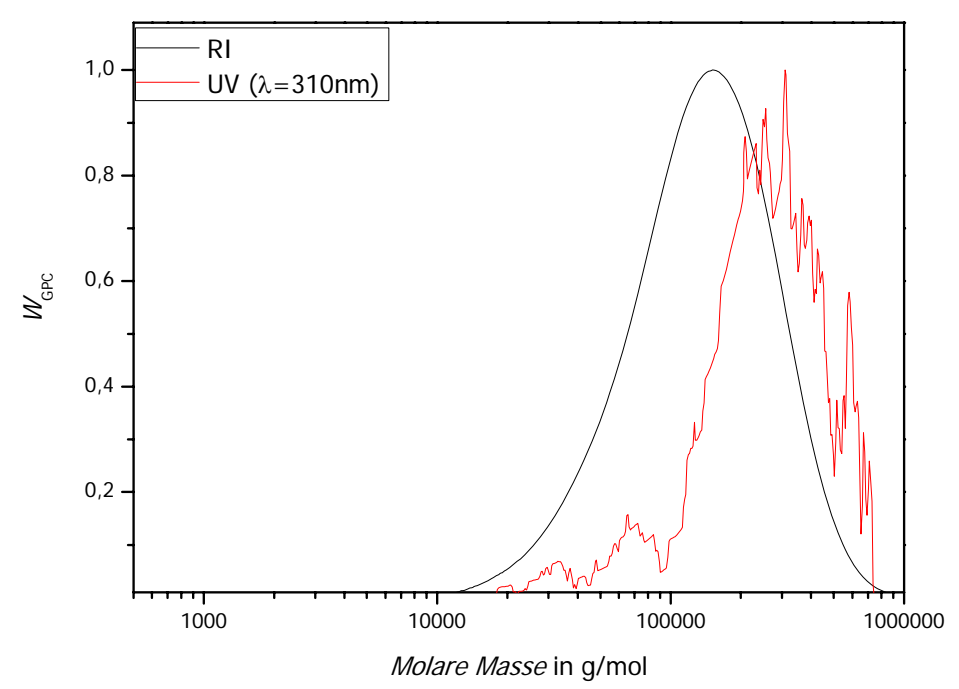

\section{Abbildung 8.11.3}

Auftragung der normierten GPC-Detektorsignale des Polymers in Lösung (PiL) gegen die mittlere Molmasse: Die RAFT-Polymerisation von der Oberfläche erzielte einen Umsatz von $U=16,21 \%$. Die schwarze Kurve beschreibt das Massendetektorsignal mit einer mittlere Molmasse von $M_{\mathrm{n}}=100500 \mathrm{~g} / \mathrm{mol}$ bei einer Polydispersität von PDI =1,66.

Trotzdem muss an dieser Stelle festgehalten werden, dass die RAFT-Polymerisation von der Rußoberfläche erfolgreich verlaufen ist. Die Reaktionskontrolle ist aufgrund der geringen Konzentration an RAFT-Agens, welches nur an der Oberfläche gebunden ist, schwach ausgeprägt aber vorhanden. Die Molekulargewichte nehmen mit dem Umsatz deutlich $\mathrm{zu}$ und es ist eine UV-Absorbanz im makromolekularen Bereich der Molekulargewichtsverteilungen in der GPC-Analyse zu erkennen. Die Zugabe von „freien“ RAFT-Agenzien in das Reaktionsgemisch würde eine sehr gute Polymerisationskontrolle erzielen, wobei dann die Herkunft der kontrollierenden Spezies unklar wäre. 
In diesem Fall allerdings kann eine Polymerisationskontrolle nur von der Rußoberfläche indiziert werden. Um das System zu verbessern, muss die effektive Konzentration an RAFT-Agens erhöht oder die Initiatorkonzentration erniedrigt werden.

\subsubsection{RAFT-Polymerisation von der Oberfläche mit „FTBPTTCP“}

In Kapitel 8.11.1 wurde bereits gezeigt, dass die RAFT-Polymerisation von der Rußoberfläche erfolgreich verlaufen ist. In diesem Kapitel soll das RAFT-Agens mit der Thioesterfunktionalität in der Ankergruppe untersucht werden. Die modifizierten Industrieruße, welche eine Beladungsdichte von $\rho_{\text {Agens }}=0,090 \mathrm{mmol} / \mathrm{g}$ aufweisen, werden hierzu in Lösungsmittelpolymerisationen von Styrol in Toluol untersucht. Tabelle 8.11.2 listet die Reaktionszusammensetzung auf. Die Konzentration an RAFT-Agens wurde im Vergleich zu vorherigen Experimenten erhöht und die Initiatorkonzentration angepasst, um eine bessere Polymerisationskontrolle zu ermöglichen.

Tabelle 8.11.2

Zusammensetzung des Polymerisationsansatzes der RAFT-Polymerisation von der Rußoberfläche mit "FTBPTTCP":

\begin{tabular}{|c|c|c|}
\hline RAFT-Agens & FTBPTTCP & $0,030 \mathrm{Mol} \%$ \\
\hline Initiator & ACC & $0,030 \mathrm{Mol} \%$ \\
\hline Monomer & Styrol & $36,5 \mathrm{Mol} \%$ \\
\hline $\begin{array}{c}\text { Lösungsmittel } \\
\text { modifizierter } \\
\text { Füllstoff }\end{array}$ & Toluol & $36,5 \mathrm{Mol} \%$ \\
\hline
\end{tabular}

Die Polymerisation wird nach Variante 2 durchgeführt. Die Trennung des Polymers in Lösung (PiL) von den Rußpartikeln erfolgt in der Zentrifuge. In Abbildung 8.11.4 ist die Entwicklung des Umsatz-Zeit-Kurve ersichtlich. Auch in diesem Fall wird die Polymerisation in der Anfangsphase inhibiert und die Umsätze steigen erst nach einer Zeit von $t=200$ min merklich an. Dies geschieht in diesem Fall allerdings schneller als für die RAFT-Polymerisation mit „FABPTTCP“ von der Rußoberfläche. Vielleicht liegt es im Rahmen der experimentellen Streuung oder der Einfluss der Rußoberfläche war unterschiedlich stark ausgeprägt. Schwankungen in den experimentellen Ergebnissen wurden ja bereits bei den TGA-Messungen identischer Proben von Industrierußen 
beobachtet (vgl. Tabelle 8.6.1) und könnten vielleicht auch den Umsatz-Zeit-Verlauf beeinflussen.

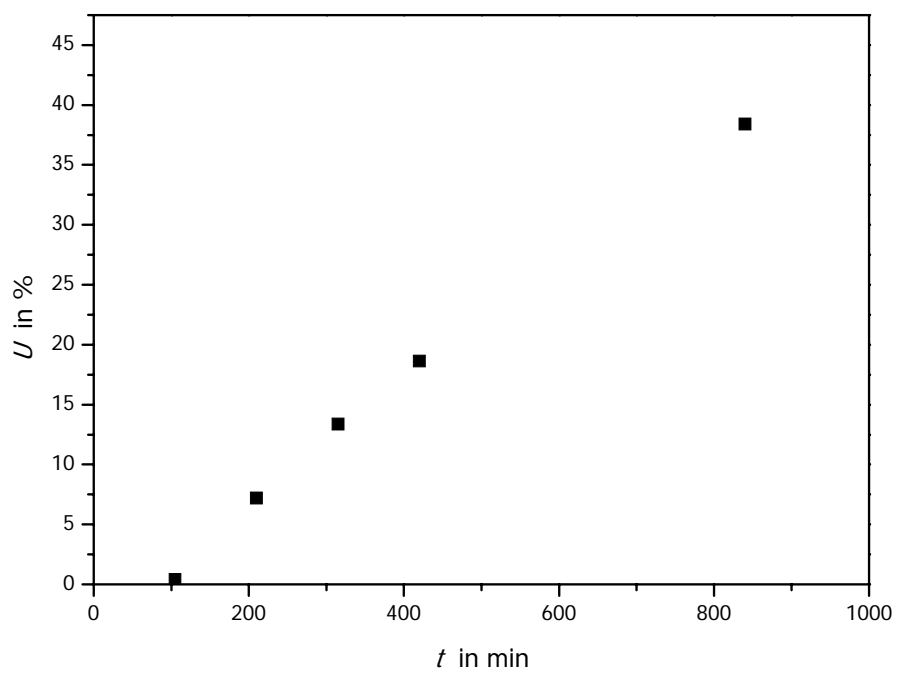

\section{Abbildung 8.11.4}

Darstellung der Umsatz-Zeit-Verläufe des Polymers in Lösung (PiL) für eine kontrollierte radikalische RAFT-Polymerisation von der Rußoberfläche mit einer Initiatorkonzentrationen von $c_{0}(A C C)=0,030 \mathrm{Mol} \%$ und einer Rußkonzentration von $c_{0}(R u \beta)=27,03 \mathrm{Mol} \%$. Die Beladungsdichte des RAFT-Agens auf der Rußoberfläche beträgt $\rho_{\text {Agens }}=0,090 \mathrm{mmol} / \mathrm{g}$, wodurch eine RAFT-Agens-Konzentration von $c_{0}($ FTBPTTCP $)=0,030 \mathrm{Mol} \%$ erreicht wird.

In Abbildung 8.11.5 ist die zeitliche Entwicklung der Molekulargewichtsverteilungen dargestellt. Es ist gut $\mathrm{zu}$ erkennen, dass sich die Verteilungen $\mathrm{zu}$ höheren Molekulargewichten verschieben, je länger die Reaktion andauert. Dieses Charakteristikum kann nur durch eine kontrollierte Polymerisation hervorgerufen werden. Da auch in diesem Fall das RAFT-Agens nur an der Rußoberfläche sitz, muss die Kontrolle an dieser Stelle hervorgerufen werden. Es ist also offensichtlich, dass die RAFT-Polymerisation erfolgreich initiiert wird. Diese Tatsache wird ebenfalls durch die Entwicklung der mittleren Molekulargewichte und der Polydispersität mit dem Umsatz in Abbildung 8.11.6 verdeutlicht. Es ist zwar weiterhin ein Hybridverhalten zu erkennen, aber der Anstieg und die Tendenz in der Entwicklung der Molekulargewichte stimmt sehr gut mit den theoretischen Vorraussagen überein. Die Polydispersität liegt in einem 
Bereich von $P D I=1,8$. Dieser Wert ist nicht aussagekräftig in Hinblick auf einen lebenden Charakter der Polymerisation.

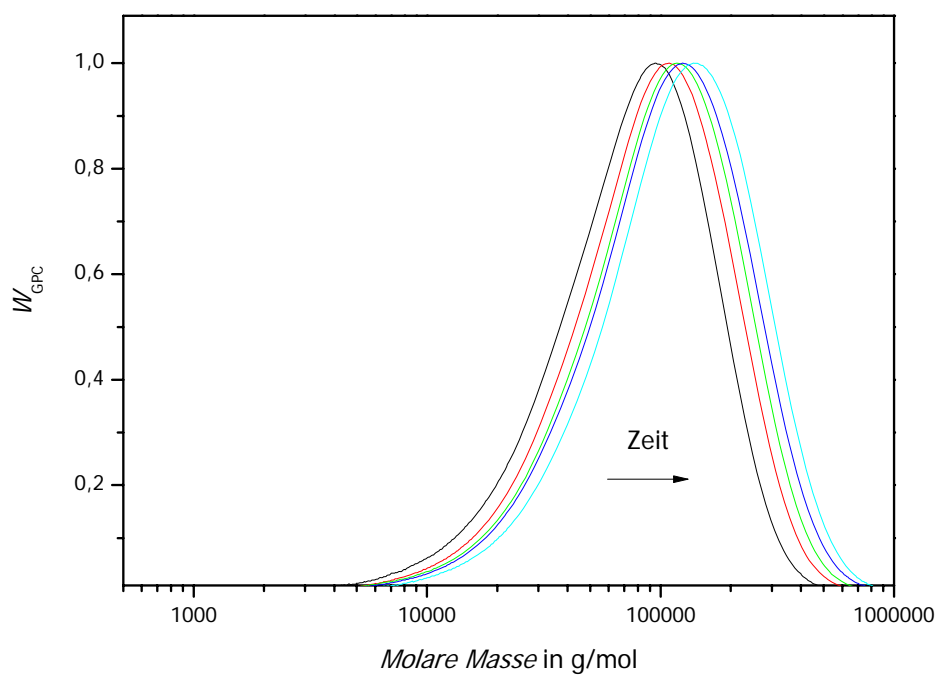

Abbildung 8.11.5

Darstellung der zeitlichen Entwicklung der normierten Molekulargewichtsverteilung der RAFT-

Polymerisation von der Rußoberfläche mit „FTBPTTCP“. Die Zusammensetzung des Polymerisationsansatzes ist in Tabelle 8.11 .2 beschrieben. 


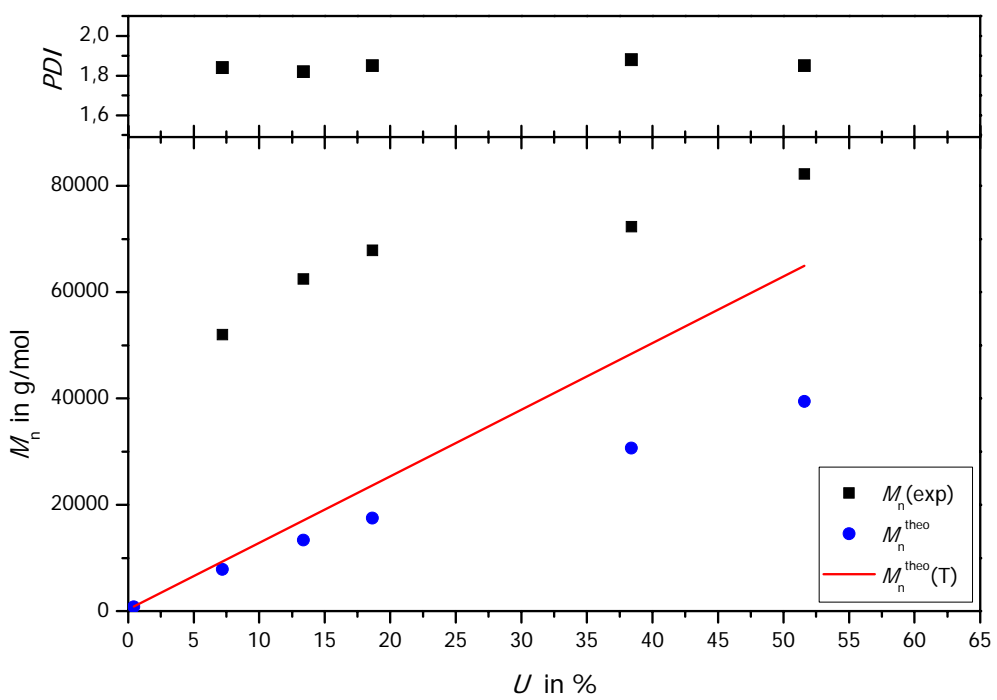

\section{Abbildung 8.11.6}

Darstellung der mittleren Molekulargewichte und der Polydispersität gegen den Umsatz:. Die rote Linie beschreibt die idealen, theoretischen mittleren Molekulargewichte. Die blauen Punkte stellen die theoretischen Molekulargewichte unter Berücksichtigung der Terminierung dar.

In Abbildung 8.11.7 werden die normierten Molekulargewichtsverteilungen des Massendetektors und des UV-Detektors verglichen. Es ist gut $\mathrm{zu}$ erkennen, dass die Absorbanz des Signals bei $\lambda=310 \mathrm{~nm}$ im makromolekularen Bereich liegt. Das Signal ist nicht so stark verrauscht und auch die Überlappung der Molekulargewichtsverteilungen ist besser. Es scheint weiniger „totes“ Material entstanden $\mathrm{zu}$ sein, als bei der vergleichbaren Polymerisation mit „FABPTTCP“ von der Rußoberfläche. Es kann davon ausgegangen werden, dass fast jeder Polymerkette eine RAFT-Funktionalität trägt und somit befähigt ist, weitere Monomereinheiten anzulagern. 


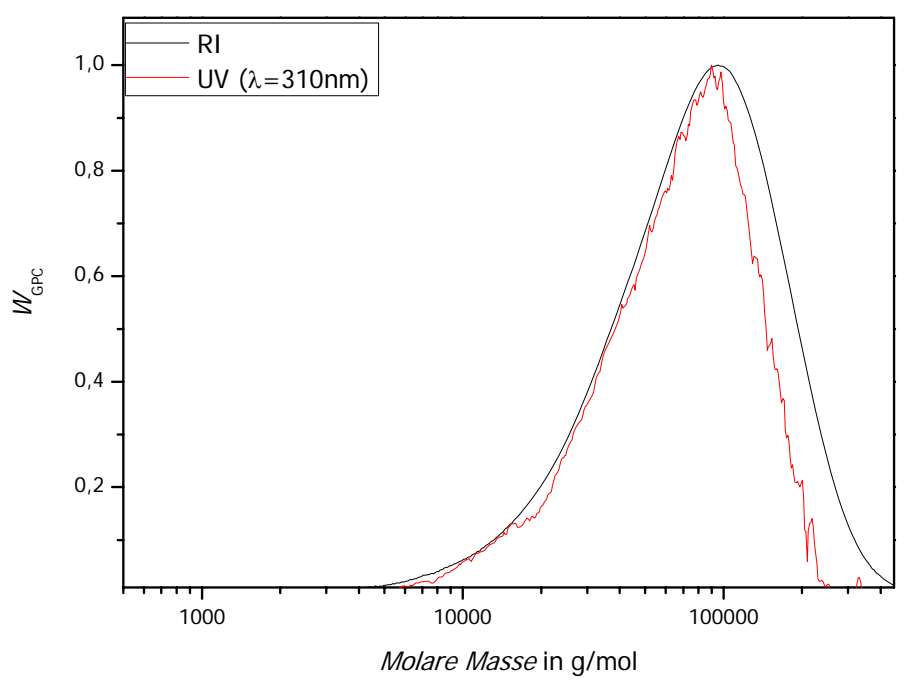

\section{Abbildung 8.11.7}

Auftragung der normierten GPC-Detektorsignale des Polymers in Lösung(PiL) gegen das mittlere Molekulargewicht: Die RAFT-Polymerisation von der Oberfläche erzielte einen Umsatz von $U=7,21 \%$. Die schwarze Kurve beschreibt das Massendetektorsignal mit einer mittlere Molmasse von $M_{\mathrm{n}}=51995 \mathrm{~g} / \mathrm{mol}$ bei einer Polydispersität von $P D I=1,82$.

Die RAFT-Polymerisation von der Rußoberfläche zeigt in dieser experimentellen Reihe eine bessere Kontrolle der Reaktion. Dieser Umstand lässt sich mit der höheren Konzentration an RAFT-Agens und der angepassten Initiatorkonzentration gut erklären. Nach der Umsatzbestimmung wird das Polymer in Lösung (PiL) vom Polymer an der Rußoberfläche (PaR) abgetrennt, um den Massenverlust des Polymers an den Rußpartikeln in der thermogravimetrischen Analyse zu untersuchen.

In Abbildung 8.11.8 ist der normierte Verlauf des Massenverlustes gegen die Temperatur für das Polymer auf der Rußoberfläche dargestellt. Es wird der Temperaturbereich von $T=300-500{ }^{\circ} \mathrm{C}$ betrachtet, in dem sich das Polymer von der Oberfläche ablöst. Da die Massenverluste in der Anfangsphase variieren (vgl. Abbildung 8.6.1) ist eine Vergleichbarkeit erschwert. Es ist gut zu erkennen, dass die Massenverluste des Polymers an der Rußoberfläche mit fortschreitender Polymerisationszeit zunehmen. Die Farben der Linien sind hierbei mit denen in Abbildung 8.11.5 identisch. Je länger die Polymerketten an der Rußoberfläche werden, umso größer fällt der Massenverlust in der thermogravimetrischen Analyse aus. 


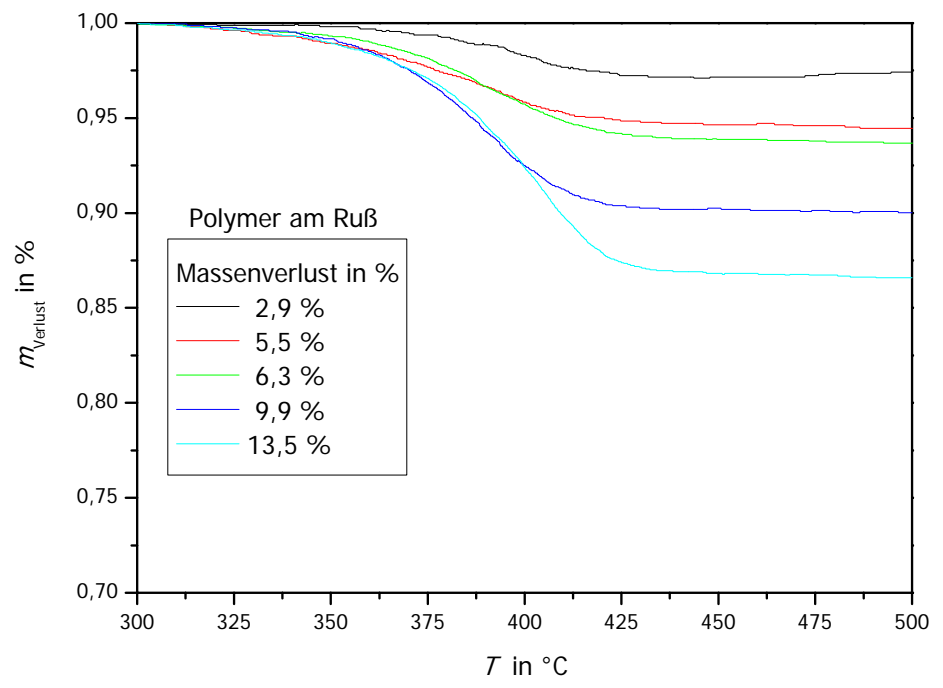

Abbildung 8.11.8

Darstellung des normierten Verlaufs des Massenverlusts gegen die Temperatur: Die Prozentangaben beziehen sich auf den effektiven Massenverlust an Polystyrol der einzelnen Rußproben (PaR). Es wird nur der für die Polymerablösung wichtige Temperaturbereich betrachtet.

Dieses Ergebnis ist bei einer erfolgreichen RAFT-Polymerisation von der Rußoberfläche zu erwarten. Die Massenverluste nehmen stetig zu. 


\subsection{Schlussfolgerung: Polymerisationen an Industrierußen}

Die Experimente zeigen, dass eine radikalische Polymerisation in Gegenwart von Industrierußen erfolgreich durchgeführt werden kann. Auch die Anbindung von RAFTAgenzien über einer Diels-Alder-Reaktion an die Oberfläche von Rußen kann erfolgreich realisiert werden. Die chemische Struktur des Rußes erlaubt die Anbindung von Furanderivaten an die Oberfläche. Hierbei hat sich gezeigt, dass die Immobilisierung einstufig oder in zwei Arbeitsschritten durchgeführt werden kann. Die Ergebnisse der Immobilisierung können mit den ausgewählten Strukturen der RAFT-Agenzien in der Elementaranalyse quantifiziert werden. Die resultierenden Beladungsdichten sind ausreichend groß, um eine anschließenden RAFT-Polymerisation ohne Zugabe von „freiem“ RAFT-Agens bei einer guten Kontrolle der Reaktion zu ermöglichen. Die ausgewählten RAFT-Agenzien zeigen eine hervorragende Kontrolle in der Lösungsmittelpolymerisation von Styrol in Toluol. Es wird gezeigt, dass die Struktur der Ankergruppe keinen Einfluss auf den Verlauf einer konventionellen radikalischen Polymerisation hat und somit durchgehend geeignet ist. Weiterhin werden die Einflüsse der Probenpräparation untersucht. Hierzu werden Polymerisationen unter Luftsauerstoff, unter Argon-Atmosphäre und nach Durchspülen mit Stickstoff in Gegenwart von Ruß untersucht. Es hat sich gezeigt, dass das Spülen mit Stickstoff ausreicht, um eine gute Polymerisationskontrolle bei der RAFT-Polymerisation zu erreichen. Diese Präparation des Polymerisationsansatzes kann sehr gut auch im größeren Maßstab im Reaktionsautoklaven umgesetzt werden.

Die Ergebnisse der Untersuchungen zeigen, dass die RAFT-Polymerisation von der Oberfläche von Industrierußen mit den vorgesehenen Agenzien und der beschriebenen Immobilisierungstechnik gut umgesetzt werden kann. Es wird ein Anstieg der mittleren Molekulargewichte mit dem Umsatz beobachtet und die Masse an Polymermaterial nimmt mit fortlaufender Polymerisationszeit zu. 


\section{Ausblick}

In dieser Arbeit wurde die Herstellung von Polybutadien und 1,3-Butadien enthaltende Copolymere mit kontrollierter Kettenstruktur durch die RAFT-Polymerisation gezielt untersucht. $\mathrm{Zu}$ diesem Zweck wurden zwei RAFT-Agenzien, exemplarisch für die Stoffklassen der Dithiobenzoate und der Trithiocarbonate, in der Copolymerisation von 1,3-Butadien mit Isopren und Butylacrylat betrachtet. Im System der Copolymerisation von 1,3-Butadien können in weiteren Arbeiten eine Vielzahl von Reaktionsparametern variiert werden. So kann der Temperaturbereich für die RAFT-Polymerisation nach oben und nach unten erweitert werden. Die erfolgreiche RAFT-Polymerisation von Isopren ${ }^{[22]}$ bei Temperaturen von $T=100{ }^{\circ} \mathrm{C}$, sowie die konventionelle radikalische Kaltpolymerisation von 1,3-Butadien geben dafür sehr gute Ansatzpunkte. Auch die Verbesserung der Reaktionskontrolle von RAFT-Polymerisationen ${ }^{[117-119]}$ unter erhöhtem Druck kann für das untersuchte System in Betracht gezogen werden. Die Verwendung von anderen RAFT-Agenzien, wie z.B. Xanthate und Dithiocarbamate in der Herstellung von statistischen Copolymeren, stellt ebenfalls eine interessante Herausforderung für vertiefende Untersuchungen dar. Neben diesem Aspekt könnten auch andere bzw. weitere Monomere Materialeigenschaften des entstehenden Synthesekautschuks verändern und somit Einfluss auf die Polarität und das Herstellungsverfahren von Gummimischungen nehmen. Als Zielsetzung weiterer Arbeiten könnten die Endumsatzsteigerung und die Erhöhung der mittleren Molekulargewichte stehen. Die Untersuchung der Emulsionspolymerisation für die Herstellung von Synthesekautschuken mit einer kontrollierten Kettenstruktur erscheint ebenfalls lohnend, da dieser Polymerisationsprozess auch großtechnisch Anwendung findet. Der in dieser Arbeit entwickelte Autoklav ist für die Umsetzung dieser Experimente bestens geeignet, da er aufgrund der Temperaturbeständigkeit und der hohen Variabilität schnell an geänderte Anforderungen angepasst werden kann.

Weiterhin wurde die RAFT-Polymerisation in Gegenwart von Industrierußen betrachtet. Erstmalig wurden die RAFT-Agenzien über eine Diels-Alder-Reaktion an die Oberfläche gebunden. Die quantitative Analyse zeigte eine erfolgreiche Immobilisierung der RAFTAgenzien. Es konnte gezeigt werden, dass die RAFT-Polymerisation mit gebundenem RAFT-Agens durchgeführt werden kann. Es ergeben sich somit eine Vielzahl von 
Prozessparametern, die weiter variiert und angepasst werden können. Die Zugabe von „freiem“ RAFT-Agens würde die Reaktionskontrolle verbessern. Auch die Variation der Polymerisationstemperatur, die Erhöhung der Beladungsdichte an RAFT-Agenzien oder die Verwendung anderer Monomere könnte die Kontrolle der RAFT-Polymerisation von der Oberfläche verbessern. Die Herstellung von Blockstrukturen oder die Synthese von statistischen Copolymeren an der Oberfläche eröffnen ein weites und hoch interessantes Forschungsfeld. So stehen jetzt neuartige Polymermaterialien in ausreichender Menge zur Verfügung, an denen grundlegende Forschungen (z.B. die elektrische und thermische Leitfähigkeit) durchgeführt werden können. Die Verwendung des so modifizierten Füllstoffs mit anderen Synthesekautschuken in Gummimischungen kann einen tiefen Einblick in die Wechselwirkung zwischen Kautschuken und Füllstoff verschaffen. 


\section{Abkürzungsverzeichnis}

ACC 1,1'-Azobis(cyclohexanecarbonitril)

$\alpha \quad$ Wachstumswahrscheinlichkeit

AM Acrylamid

AN Acrylnitirl

ATRP Atom Transfer Radical Polymerization

BA Butylacrylat

BD 1,3-Butadien

BPETC Bis(1-phenylethyl)trithiocarbonat

BR Polybutadien

bzw. beziehungsweise

$c \quad$ Konzentration

CDB Cumyldithiobenzoat

$c_{\mathrm{R}} \quad$ Makroradikalkonzentration

$C_{\text {tr }} \quad$ Übertragungskonstante

$d \quad$ Anzahl bei der Terminierung gebildeten Polymerketten

d.h. das heißt

DBE Dibenzylether

DMSO Dimethylsulfoxid

eq. Äquivalent

$f \quad$ Initiatoreffektivität

FABP Furfuryl-2-bromopropanoat

FABPTTCP Propyl-(furfurylpropanioat)-trithiocarbonat

FTBP Furfuryl-2-bromopropanthioat

FTBPTTCP Propyl-(furfurylpropanthioat)-trithiocarbonat

GPC Gel-Permeations-Chromatographie

IP Isopren

IR Polyisoprenkautschuk

$k_{\text {ad }} \quad$ Geschwindigkeitskoeffizient der Addition

$k_{\text {akt }} \quad$ Geschwindigkeitskoeffizient der Aktivierung

$k_{\mathrm{d}} \quad$ Geschwindigkeitskoeffizient des Initiatorzerfalls 
$k_{\text {deakt }} \quad$ Geschwindigkeitskoeffizient der Deaktivierung

$k_{\mathrm{fr}}$

$k_{\mathrm{k}}$

$k_{\mathrm{p}}$

$k_{\mathrm{p}, 1}$

$k_{\mathrm{t}}$

$k_{\mathrm{t}, \mathrm{d}}$

$k_{\mathrm{t}, \text { int-int }}$

$k_{\mathrm{t}, \mathrm{int}-\mathrm{R}}$

$k_{\mathrm{t}, \mathrm{k}}$

$k_{\mathrm{tr}}$

LDPE

Lsg.

M

MA

MADIX

$\max$.

$M_{\mathrm{i}}$

$m_{\mathrm{i}}$

MMA

$M_{\mathrm{n}}$

$M_{\mathrm{n}}^{\text {theo }}$

$M_{\mathrm{n}}^{\text {theo }}(\mathrm{T})$

$m_{\text {Verlust }}$

$M_{\mathrm{w}}$

$N_{\mathrm{i}}$

NMP

NMR

NR

NVC

NVP
Geschwindigkeitskoeffizient der Fragmentierung

Geschwindigkeitskoeffizient der Kombination

Geschwindigkeitskoeffizient der Propagation

Geschwindigkeitskoeffizient des ersten Propagationsschritts

Geschwindigkeitskoeffizient der Terminierung

Geschwindigkeitskoeffizient der Terminierung nach Disproportionierung

Geschwindigkeitskoeffizient der Terminierung zweier Intermediate

Geschwindigkeitskoeffizient der Terminierung des Intermediats mit einem Makroradikal

Geschwindigkeitskoeffizient der Terminierung nach Kombination

Geschwindigkeitskoeffizient von

Polyethylen niedriger Dichte

Lösung

Monomereinheiten

Methacrylat

Macromolecular Design via the Interchange of Xanthates

maximal

spezifisches Molekulargewicht eines Makromoleküls

Gesamtmasse aller Makromoleküle mit spezifischen Molekulargewicht

Methylmethacrylat

Zahlenmittelwert der Molmasse (mittleres Molekulargewicht

Theoretisches mittleres Molekulargewicht

heoretisches mittleres Molekulargewicht unter Berücksichtigung der

Terminierung

Massenverlust

Gewichtsmittelwert der Molmasse

Anzahl der Makromoleküle mit dem Molekulargewicht $M_{\mathrm{i}}$

Nitroxide Mediated Polymerization

Kernmagnetische Resonanzspektroskopie

Naturkautschuk

$\mathrm{N}$-Vinylcarbazol

$\mathrm{N}-$ Vinylpyrrolidon 
$\varnothing$

PBA

PBD

PDI

PFA

PS

PTFE

PVC

$\mathrm{R}$

RAFT

$R_{\mathrm{d}}$

RI

$R_{\mathrm{p}}$

RS

$R_{\mathrm{t}}$

RT

$R_{\text {tr }}$

RZ

$\rho$

SBR

$\delta$

$\mathrm{St}$

TEMPO

TGA

THF

TTCP

$U$

$U_{\mathrm{P}}$

usw.

UV

VAc

vgl.

$w_{\mathrm{i}}$
Durchmesser

Polybutylacrylat

Polybutadien

Polydispersitätsindex

Perfluoralkoxy

Polystyrol

Polytetrafluorethylen

Polyvinylchlorid

Radikal

Reversible Addition-Fragmentation chain Transfer

Radikalbildungsgeschwindigkeit

Brechungsindex (Refractive Index)

Polymerisationsgeschwindigkeit

Rohrstück

Kettenabbruchsgeschwindigkeit

Raumtemperatur

Geschwindigkeit der Transferreaktion

Reduzierstück

Beladungsdichte

Styrol-Butadien-Kautschuk

chemische Verschiebung

Styrol

2,2,6,6-Tetramethylpiperidinoxyl

Thermogravemetrie

Tetrahydrofuran

Natrium-(propyl)-trithiocarbonat

Umsatz

Partialumsatz

und so weiter

Ultraviolett

Vinylacetat

vergleiche

Massenanteil der Makromoleküle mit Molekulargewicht $M_{\mathrm{i}}$ 


$\begin{array}{ll}x_{P}, & \text { Wahrscheinlichkeit der Kettenlänge } P \\ x_{P, \mathrm{c}} & \text { Wahrscheinlichkeit der Kettenlänge } P \text { bei der Kombination } \\ x_{P, \mathrm{~d}} & \text { Wahrscheinlichkeit der Kettenlänge } P \text { bei der Disproportionierung } \\ \text { z.B. } & \text { zum Beispiel }\end{array}$




\section{Literaturverzeichnis}

[1] H. G. Elias, "Makromoleküle", Wiley-VCH, 1999.

[2] M. Szwarc, Nature 1956, 176, 1168.

[3] A. A. Korotkov, A. F. Podolsky, Journal of Polymer Science Part B-Polymer Letters 1965, 3, 901 .

[4] K. Matyjaszewski, J. L. Wang, T. Grimaud, D. A. Shipp, Macromolecules 1998, 31, 1527.

[5] N. Ide, T. Fukuda, Macromolecules 1999, 32, 95.

[6] N. Ide, T. Fukuda, Macromolecules 1997, 30, 4268.

[7] H. Schlaad, B. Schmitt, A. H. E. Muller, Angewandte Chemie-International Edition 1998, 37, 1389.

[8] H. Fischer, Chem. Rev. 2001, 101, 3581.

[9] H. Fischer, Macromolecules 1997, 30, 5666.

[10] E. Rizzardo, J. Chiefari, B. Y. K. Chong, F. Ercole, J. Krstina, J. Jeffery, T. P. T. Le, R. T. A. Mayadunne, G. F. Meijs, C. L. Moad, G. Moad, S. H. Thang, Macromol. Symp. 1999, 143, 291.

[11] R. T. A. Mayadunne, E. Rizzardo, J. Chiefari, Y. K. Chong, G. Moad, S. H. Thang, Macromolecules 1999, 32, 6977.

[12] C. Barner-Kowollik, P. Vana, T. P. Davis, K. Matyjaszewski, "Radical Polymerization" in "Encyclopedia of Polymer Science and Engeneering", 2003.

[13] M. Buback, Makromolekulare Chemie-Macromolecular Chemistry and Physics 1990, 191, 1575.

[14] R. D. Gilbert, H. L. Williams, J. Am. Chem. Soc. 1952, 74, 4114.

[15] E. Ozizmir, G. Odian, J. Polym. Sci. Pol. Chem. 1980, 18, 2281.

[16] H. Jacobson, W. H. Stockmayer, J. Chem. Phys. 1950, 18, 1600.

[17] G. Moad, J. Chiefari, Y. K. Chong, J. Krstina, R. T. A. Mayadunne, A. Postma, E. Rizzardo, S. H. Thang, Polym. Int. 2000, 49, 993.

[18] Y. K. Chong, T. P. T. Le, G. Moad, E. Rizzardo, S. H. Thang, Macromolecules 1999, $32,2071$.

[19] Y. K. Chong, J. Krstina, T. P. T. Le, G. Moad, A. Postma, E. Rizzardo, S. H. Thang, Macromolecules 2003, 36, 2256. 
[20] J. Chiefari, R. T. A. Mayadunne, C. L. Moad, G. Moad, E. Rizzardo, A. Postma, M. A. Skidmore, S. H. Thang, Macromolecules 2003, 36, 2273.

[21] C. Barner-Kowollik, M. Buback, B. Charleux, M. L. Coote, M. Drache, T. Fukuda, A. Goto, B. Klumperman, A. B. Lowe, J. B. McLeary, G. Moad, M. J. Monteiro, R. D. Sanderson, M. P. Tonge, P. Vana, J. Polym. Sci. Pol. Chem. 2006, 44, 5809.

[22] V. Jitchum, S. Perrier, Macromolecules 2007, 40, 1408.

[23] R. Rotzoll, P. Vana, J. Polym. Sci. Pol. Chem. 2008, 46, 7656.

[24] N. Aoyagi, B. Ochiai, H. Mori, T. Endo, Synlett 2006, 636.

[25] M. H. Stenzel, L. Cummins, G. E. Roberts, T. P. Davis, P. Vana, C. BarnerKowollik, Macromol. Chem. Phys. 2003, 204, 1160.

[26] D. B. Hua, J. X. Zhang, R. Bai, W. Q. Lu, C. Y. Pan, Macromol. Chem. Phys. 2004, $205,1125$.

[27] J. H. Zhang, A. J. Dong, T. Y. Cao, R. W. Guo, Eur. Polym. J. 2008, 44, 1071.

[28] Y. Kwak, A. Goto, Y. Tsujii, Y. Murata, K. Komatsu, T. Fukuda, Macromolecules 2002, 35, 3026 .

[29] C. Barner-Kowollik, J. F. Quinn, D. R. Morsley, T. P. Davis, J. Polym. Sci. Pol. Chem. 2001, 39, 1353.

[30] C. Barner-Kowollik, J. F. Quinn, T. L. U. Nguyen, J. P. A. Heuts, T. P. Davis, Macromolecules 2001, 34, 7849.

[31] M. J. Monteiro, H. de Brouwer, Macromolecules 2001, 34, 349.

[32] M. L. Coote, L. Radom, J. Am. Chem. Soc. 2003, 125, 1490.

[33] M. L. Coote, E. I. Izgorodina, E. H. Krenske, M. Busch, C. Barner-Kowollik, Macromol. Rapid Commun. 2006, 27, 1015.

[34] Y. Kwak, A. Goto, T. Fukuda, Macromolecules 2004, 37, 1219.

[35] T. Arita, M. Buback, P. Vana, Macromolecules 2005, 38, 7935.

[36] M. Buback, P. Vana, Macromol. Rapid Commun. 2006, 27, 1299.

[37] M. Buback, O. Janssen, R. Oswald, S. Schmatz, P. Vana, Macromol. Symp. 2007, $248,158$.

[38] O. F. Olaj, I. Bitai, F. Hinkelmann, Makromolekulare Chemie-Macromolecular Chemistry and Physics 1987, 188, 1689.

[39] B. Springer, "Polybutadien und Butadien-Styrol-Copolymere mit kontrollierter Kettenstruktur durch RAFT-Polymerisation", Georg-August-Universität, Göttingen, 2007. [40] R. Lakshmi, S. K. Athithan, Polym. Compos. 1999, 20, 346. 


\section{Literaturverzeichnis}

[41] W. Brown, R. M. Johnsen, C. Konak, L. Dvoranek, J. Chem. Phys. 1992, 96, 6274.

[42] M. K. Baloch, A. Rauf, Makromolekulare Chemie-Macromolecular Chemistry and Physics 1988, 189, 1517.

[43] P. Roychowdhury, V. D. Deuskar, J. Appl. Polym. Sci. 1986, 31, 145.

[44] P. Roychowdhury, V. D. Deuskar, J. Appl. Polym. Sci. 1984, 29, 153.

[45] M. C. Williams, Aiche J. 1975, 21, 1.

[46] L. A. Utracki, R. Simha, L. J. Fetters, Journal of Polymer Science Part B-Polymer Physics 1968, 6, 2051.

[47] G. R. Seely, Aiche J. 1964, 10, 56.

[48] "www.dguv.de/ifa/de/gestis/stoffdb/index.jsp", Institut für Arbeitsschutz der Deutschen Gesetzlichen Unfallversicherung, 2011.

[49]

"www.wittgas.com/filepool/Datenblaetter/deutsch/armaturen/flammensperre f53n rf53n es d.pdf", WITT-GASETECHNIK GmbH \& Co KG, 2011.

[50] "www.swagelok.de/downloads/WebCatalogs/DE/MS-01-140.PDF", 2011 Swagelok Company.

[51] R. Z. Wei, Y. W. Luo, Z. S. Li, Polymer 2010, 51, 3879.

[52] S. Jouenne, J. A. Gonzalez-Leon, A. V. Ruzette, P. Lodefier, S. Tence-Girault, L. Leibler, Macromolecules 2007, 40, 2432.

[53] J. W. Zhao, H. Zhu, Y. X. Wu, R. Jian, G. Y. Wu, Chin. J. Polym. Sci. 2010, 28, 385.

[54] J. E. Kennedy, J. G. Lyons, L. M. Geever, C. L. Higginbotham, Mater. Sci. Eng. CBiomimetic Supramol. Syst. 2009, 29, 1655.

[55] N. Saetung, I. Campistron, S. Pascual, J. F. Pilard, L. Fontaine, Macromolecules 2011, 44, 784 .

[56] G. Bar-Nes, R. Hall, V. Sharma, M. Gaborieau, D. Lucas, P. Castignolles, R. G. Gilbert, Eur. Polym. J. 2009, 45, 3149.

[57] D. S. Germack, K. L. Wooley, Macromol. Chem. Phys. 2007, 208, 2481.

[58] A. Kaiser, S. Brandau, M. Klimpel, C. Barner-Kowollik, Macromol. Rapid Commun. 2010, 31, 1616.

[59] C. Barner-Kowollik, S. Perrier, J. Polym. Sci. Pol. Chem. 2008, 46, 5715.

[60] L. Barner, T. P. Davis, M. H. Stenzel, C. Barner-Kowollik, Macromol. Rapid Commun. 2007, 28, 539. 
[61] J. Chiefari, Y. K. Chong, F. Ercole, J. Krstina, J. Jeffery, T. P. T. Le, R. T. A. Mayadunne, G. F. Meijs, C. L. Moad, G. Moad, E. Rizzardo, S. H. Thang, Macromolecules 1998, 31, 5559.

[62] J. Hua, H. B. Xu, J. T. Geng, Z. F. Deng, L. Xu, Y. L. Yu, J. Polym. Res. 2011, 18, 41.

[63] J. Hua, J. T. Geng, X. Wang, J. Zhao, L. Xu, J. Macromol. Sci. Part A-Pure Appl. Chem. 2009, 46, 1156.

[64] G. Morandi, S. Pascual, V. Montembault, S. Legoupy, N. Delorme, L. Fontaine, Macromolecules 2009, 42, 6927.

[65] S. U. Heo, G. H. Rhee, D. H. Lee, J. H. Kim, D. S. Choi, D. W. Lee, J. Ind. Eng. Chem. 2006, 12, 241.

[66] "Messungen der Continental Reifen GmbH", Hannover, 2008.

[67] M. Kamachi, A. Kajiwara, Macromolecules 1996, 29, 2378.

[68] F. Bandermann, C. Gunther, J. Schweer, Macromol. Chem. Phys. 1996, 197, 1055.

[69] K. Winnacker, L. Küchler, R. Dittmeyer, "Chemische Technik - Prozesse und Produkte", 2005.

[70] F. Ullmann, M. Bohnet, "Ullmann's encyclopedia of industrial chemistry", 2003.

[71] J. Kroschwitz, M. Howe-Grant, "Kirk-Othmer-Encyclopedia of chemical technology", 4th edition, 1998.

[72] T. Q. Liu, S. J. Jia, T. Kowalewski, K. Matyjaszewski, R. Casado-Portilla, J. Belmont, Macromolecules 2006, 39, 548.

[73] K. H. Kuo, W. Y. Chiu, T. M. Don, J. Appl. Polym. Sci., 115, 1803.

[74] R. A. Casado, P. A. Lovell, P. Navabpour, J. L. Stanford, Polymer 2007, 48, 2554.

[75] T. Q. Liu, S. Jia, T. Kowalewski, K. Matyjaszewski, R. Casado-Portilla, J. Belmont, Langmuir 2003, 19, 6342.

[76] S. Ababou-Girard, F. Solal, B. Fabre, F. Alibart, C. Godet, J. Non-Cryst. Solids 2006, $352,2011$.

[77] H. Y. Xu, J. J. Han, L. Fang, F. Shen, C. F. Wu, Polym. Bull. 2007, 58, 951.

[78] D. Yang, G. Q. Guo, J. H. Hu, C. Wang, D. L. Jiang, J. Mater. Chem. 2008, 18, 350.

[79] R. R. Nayak, A. M. Shanmugharaj, S. H. Ryu, Macromol. Chem. Phys. 2008, 209, 1137.

[80] W. J. Huang, S. Fernando, L. F. Allard, Y. P. Sun, Nano Lett. 2003, 3, 565. 


\section{Literaturverzeichnis}

[81] Z. L. Yao, N. Braidy, G. A. Botton, A. Adronov, J. Am. Chem. Soc. 2003, 125, 16015.

[82] D. Tasis, N. Tagmatarchis, A. Bianco, M. Prato, Chem. Rev. 2006, 106, 1105.

[83] N. Karousis, N. Tagmatarchis, D. Tasis, Chem. Rev. 2010, 110, 5366.

[84] Q. Yang, L. Wang, W. D. Xiang, J. F. Zhou, G. H. Jiang, J. Appl. Polym. Sci. 2007, 103, 2086.

[85] D. Borah, S. Satokawa, S. Kato, T. Kojima, Appl. Surf. Sci. 2008, 254, 3049.

[86] T. Takada, M. Nakahara, H. Kumagai, Y. Sanada, Carbon 1996, 34, 1087.

[87] K. Horita, Y. Nishibori, T. Ohshima, Carbon 1996, 34, 217.

[88] E. Saab, S. Aouad, E. Abi-Aad, M. N. Bokova, E. A. Zhilinskaya, A. Aboukais, Kinet. Catal. 2007, 48, 841.

[89] J. F. Huang, F. Shen, X. H. Li, X. Q. Zhou, B. Y. Li, R. L. Xu, C. F. Wu, J. Colloid Interface Sci. 2008, 328, 92.

[90] J. Ueda, H. Yamaguchi, T. Yamauchi, N. Tsubokawa, J. Polym. Sci. Pol. Chem. 2007, $45,1143$.

[91] G. Sakellariou, H. N. Ji, J. W. Mays, D. Baskaran, Chem. Mat. 2008, 20, 6217.

[92] S. Hayashi, S. Handa, N. Tsubokawa, J. Polym. Sci. Pol. Chem. 1996, 34, 1589.

[93] T. Q. Liu, R. Casado-Portilla, J. Belmont, K. Matyjaszewski, J. Polym. Sci. Pol. Chem. 2005, 43, 4695.

[94] Q. Yang, L. Wang, J. Huo, J. H. Ding, W. D. Xiang, J. Appl. Polym. Sci. 2009, 117, 824.

[95] Q. Yang, L. Wang, W. Xiang, J. Zhou, Q. h. Tan, Polymer 2007, 48, 3444.

[96] G. Y. Xu, W. T. Wu, Y. S. Wang, W. M. Pang, Q. R. Zhu, P. H. Wang, Y. Z. You, Polymer 2006, 47, 5909.

[97] G. Y. Xu, Y. S. Wang, W. M. Pang, W. T. Wu, Q. R. Zhu, P. H. Wang, Polym. Int. 2007, 56, 847.

[98] D. H. Nguyen, P. Vana, Polymers for Advanced Technologies 2006, 17, 625.

[99] L. Zhang, J. Z. Yang, C. L. Edwards, L. B. Alemany, V. N. Khabashesku, A. R. Barron, Chem. Commun. 2005, 3265.

[100] F. M. Fernandes, R. Araujo, M. F. Proenca, C. J. R. Silva, M. C. Paiva, Journal of Nanoscience and Nanotechnology 2007, 7, 3514.

[101] M. T. Beck, G. Mandy, S. Papp, I. Dekany, Colloid Polym. Sci. 2004, 283, 237. 
[102] T. Laue, A. Plagens, "Namen- und Schlagwort-Reaktionen der Organischen Chemie", 5th edition, 2006.

[103] X. Lu, F. Tian, N. Q. Wang, Q. N. Zhang, Org. Lett. 2002, 4, 4313.

[104] J. L. Delgado, P. de la Cruz, F. Langa, A. Urbina, J. Casado, J. T. L. Navarrete, Chem. Commun. 2004, 1734.

[105] C. Menard-Moyon, F. Dumas, E. Doris, C. Mioskowski, J. Am. Chem. Soc. 2006, $128,14764$.

[106] C. M. Chang, Y. L. Liu, Carbon 2009, 47, 3041.

[107] S. Pine, J. B. Hendrickson, D. J. Cram, G. S. Hammond, "Organische Chemie", 4th edition, 1987.

[108] X. P. Qiu, F. M. Winnik, Macromol. Rapid Commun. 2006, 27, 1648.

[109] S. Jana, A. Parthiban, C. L. L. Chai, J. Polym. Sci. Pol. Chem. 2011, 49, 1494.

[110] S. Munirasu, J. Albuerne, A. Boschetti-de-Fierro, V. Abetz, Macromol. Rapid Commun. 2010, 31, 574.

[111] Y. K. Yang, Z. F. Yang, Q. Zhao, X. J. Cheng, S. C. Tiong, R. K. Y. Li, X. T. Wan, X. L. Xie, J. Polym. Sci. Pol. Chem. 2009, 47, 467.

[112] Y. Zhao, S. Perrier, Macromolecules 2007, 40, 9116.

[113] M. D. Rowe-Konopacki, S. G. Boyes, Macromolecules 2007, 40, 879.

[114] R. Ranjan, W. J. Brittain, Macromolecules 2007, 40, 6217.

[115] K. Y. Sui, C. J. Yang, S. Gao, X. Shan, Y. Z. Xia, Q. Zheng, J. Appl. Polym. Sci. 2009, 114, 1914.

[116] K. Ohkita, N. Tsubokawa, E. Saitoh, M. Noda, N. Takashina, Carbon 1975, 13, 443.

[117] M. Buback, W. Meiser, P. Vana, Aust. J. Chem. 2009, 62, 1484.

[118] T. Arita, M. Buback, O. Janssen, P. Vana, Macromol. Rapid Commun. 2004, 25, 1376.

[119] M. J. Monteiro, R. Bussels, S. Beuermann, M. Buback, Aust. J. Chem. 2002, 55, 433. 


\section{Danksagung}

Herrn Prof. Dr. Philipp Vana, MBA danke ich für die interessante Themenstellung, die lehrreichen Diskussionen, die großartige Unterstützung und die stete Förderung dieser Arbeit.

Bei Herrn Prof. Michael Buback möchte ich mich für die gute Betreuung und die vielen lebensnahen Ratschläge bedanken.

Der Continental Reifen $\mathrm{GmbH}$ danke ich für die finanzielle Unterstützung meiner Promotion und für die lehrreichen und unterhaltsamen Treffen im Rahmen der Kooperation. Besonders Frau Dr. Sabine Müller, Frau Dr. Lena Müller, Frau Dr. Katharina Herzog und Frau Dr. Carla Recker möchte ich für die erfolgreiche Zusammenarbeit danken.

Volker Meyer, Andreas Knorr, Dietmar Wüstefeld und allen weiteren Mitarbeitern der

Werkstätten gilt mein Dank für die gewissenhafte und schnelle Umsetzung von Fertigungs- und Reparaturaufträgen und die tatkräftige Unterstützung in der Fertigung des experimentellen Aufbaus.

Weiterhin danke ich Cathrin und Nadja für die angenehme und erfolgreiche Zusammenarbeit im Laboralltag. An dieser Stelle möchte ich ebenfalls Denis, Robert und Pascal für die moralische Unterstützung in frustrierenden Momenten und allen Mitgliedern der Abteilungen von Prof. Vana und Prof. Buback für die angenehme und schöne Arbeitsatmosphäre danken.

Ein großer Dank gilt auch Ines und den Jungs aus der Ewaldstraße für die Unterstützung, den gehabten Spaß und einfach für die schönen Dinge neben dem Studium. Ihr habt mich immer an das Leben neben der Chemie erinnert.

Meinen Eltern gebührt mein tiefster Dank für die moralische und finanzielle Unterstützung während des gesamten Studiums und der Doktorarbeit.

Zum Abschluss möchte ich mich bei meiner Freundin Katarina für ihre Geduld und die Unterstützung bedanken. 


\section{Lebenslauf}

\section{Björn Springer}

\section{Persönliche Daten}

geb. am 12.06.1981 in Berlin-Buch

Deutsch

ledig

\section{Hochschulstudium}

seit $09 / 07$

Anfertigung der Dissertation im Arbeitskreis von Prof. Dr. P. Vana. Titel: „Polybutadien und Butadien enthaltene Copolymere mit kontrollierter Kettenstruktur durch RAFTPolymerisation“"

$07 / 07$

Diplomprüfung

$11 / 06-05 / 07$

Anfertigung der Diplomarbeit im Arbeitskreis von Prof. Dr. M. Buback. Titel: „Polybutadien und Butadien-Styrol-Copolymere durch kontrollierte radikalische Polymerisation“

$10 / 04$

Diplomvorprüfung

$10 / 02-07 / 07$

Studium der Chemie an der Georg-August-Universität Göttingen

\section{Wehrdienst / Schulbildung}

$09 / 01-06 / 02$

$07 / 01$

$09 / 90-07 / 01$

$09 / 88-07 / 90$
Erfüllung der Wehrpflicht im 3./PzFlakBtl 131 in Hohenmölsen

Abitur am Johann-Gottfried-Herder-Gymnasium für Fremdsprachen in Berlin-Lichtenberg

Johann-Gottfried-Herder-Gymnasium für Fremdsprachen in Berlin-Lichtenberg

2.Grundschule Berlin-Weißensee 